\title{
A Controlled Trial of Chinese Herbal Medicine for Premenstrual Syndrome
}

\author{
Patsy Bin-Yo Chou
}

2007

A thesis submitted in fulfilment of the Degree of

\section{Doctor of Philosophy}

\author{
School of Health Sciences
}

Faculty of Health, Engineering and Science

Victoria University

Melbourne, Australia 


\begin{abstract}
Premenstrual syndrome is a common disorder troubling many women during their reproductive years. The Chinese have been using herbal medicines to treat menstrual cycle related symptoms for centuries. Traditional Chinese medicine provides the aetiology and pathogenesis of the symptoms of this disorder by applying Pattern Identification to diagnose the signs and symptoms of individual patients and to design individualised treatments which seek to address the entire pattern/s of disharmony. It follows that PMS, which has many symptoms and an idiosyncratic nature, is precisely an area for which Chinese medicine is most suitable. The aim of this study is to scientifically evaluate the effectiveness of Chinese herbal medicine on Australian women for the treatment of PMS within the theoretical frame-work of TCM. A double-blind randomized placebo-controlled crossover clinical trial was conducted by a qualified Chinese herbal practitioner and pharmacist. Following two months confirmatory assessment, sixty-one subjects were assigned randomly into two groups within different TCM patterns of Liver Qi Stagnation, Liver and Kidney Yin Deficiency, Liver and Spleen Disharmony and Heart and Spleen Deficiency. Herbal medicine $(n=31)$ and placebo $(n=30)$ were provided sequentially for a period of three months. There were significant differences ( $p$ $<.01)$ in scores after three months of treatment between Chinese herbal medicine and placebo in premenstrual physical and psychological symptoms, depression, anxiety and anger, but with no difference in perceived stress ( $\mathrm{p}$ $>.05)$. There were highly significant reductions $(\mathrm{p}<.001)$ in all assessments in both groups except that a significant result $(\mathrm{p}<.05)$ was recorded on perceived
\end{abstract}


stress only in the treatment-first group between baseline and the end of the third herbal treatment month. Also, there was no significant difference on treatment effects shown between TCM patterns. No adverse effects were reported by any participant. The results support the hypothesis that the symptoms occurrence and severity of PMS can be effectively reduced by the use of Chinese herbal medicine. On this basis, it is considered that Chinese herbal medicine may offer an alternative treatment method to western drugs for PMS sufferers who either do not accept the current treatment options or are having unwanted side-effects with their current treatment. 


\section{Declaration}

"I, Patsy Bin-Yo Chou, declare that the PhD thesis entitled A Controlled Trial of Chinese Herbal Medicine for Premenstrual Syndrome is no more than 100,000 words in length, exclusive of tables, figures, appendices, references and footnotes. This thesis contains no material that has been submitted previously, in whole or in part, for the award of any other academic degree or diploma. Except where otherwise indicated, this thesis is my own work". 


\section{Acknowledgments}

I am most grateful to my principal supervisor, Professor Carol Morse, for her constant support, guidance, and encouragement throughout the entire course of this study, and for her advice in the preparation of the clinical trial and drafting of this thesis.

I would also like to thank Dr Xu Hong, my co-supervisor, for her assistance and support during the study.

My sincere thanks also extend to:

Ms Maja Rechsteiner and Mr Chris Mills, for their contribution in the preparation of herbal medicine.

Ms Pei Shan Wang and all the students in the King Street clinic, for their assistance and co-operation.

Ms Dawn Bannon, for her help in arranging consultation rooms and ordering herbal medicine.

Sun Ten and Meridian Herbals for providing discounts on herbal granules.

Finally, I would like to express my gratitude to my husband Peter for his unwavering support, patience and proof reading of my drafts, to my daughter Eugenia for her support and encouragement, and my deceased father who taught me how to deal with challenges patiently, systematically and wisely. 
Table of Contents

Abstract

Declaration

Acknowledgments

Table of Contents

List of Tables

List of Figures

List of Appendices

Section I: Introduction of the thesis

Chapter 1: Introduction

Section II: Literature Review

Chapter 2: Western Biomedical Perspective on Issues for Premenstrual Syndrome

Chapter 3: Traditional Chinese Medical Perspective on Issues for Premenstrual Syndrome

Chapter 4: Methodological Issues for Research on Premenstrual Syndrome

Chapter 5: A Review of Clinical Research of Premenstrual Syndrome Treatment Using Herbal Medicine in Chinese Medical Journals 1996-2005

Section III: Empirical Research Study

Chapter 6: Objectives, Significances and Hypotheses of the Study

Chapter 7: Methodology of the Intervention Study Using Chinese

Herbal Medicine vs. Placebo

Chapter 8: Descriptive Findings of Assessment at Baseline

Chapter 9: Multivariate Analyses of Group Treatments

Chapter 10: Individual Symptoms Ratings, TCM Issues and Others

Chapter 12: Conclusions, Recommendations and Future Direction 


\section{List of Tables}

Table 2.1 The order and frequency of the most common PMS

Page symptoms in different countries

Table 2.2 MDQ Subscales (Moo's 1968) 28

Table 2.3 Subgroups in Abraham's Menstrual Symptom Diary 29

$\begin{array}{llr}\text { Table 3.1 22 TCM Patterns of PMS } & 81\end{array}$

$\begin{array}{lll}\text { Table 3.2 Pharmacological effects of liver coursing herbs } & 115\end{array}$

Table 3.3 Pharmacological effects of liver clearing and heat 116

$\begin{array}{lll}\text { Table 3.4 Pharmacological effects of liver and kidney nourishing } & 117\end{array}$ herbs

$\begin{array}{lll}\text { Table 3.5 Pharmacological effects of spleen strengthening herbs } & 118\end{array}$

$\begin{array}{lll}\text { Table 3.6 Pharmacological effects of blood moving and stasis } & 119\end{array}$

Table 5.1 Premenstrual syndrome or symptoms studied at the $124 \quad 149$ trials

$\begin{array}{lll}\text { Table 5.2 Samples sizes of the } 124 \text { trials } & 150\end{array}$

Table 5.3 Total effective rates of 21 trials 155

Table 5.4 Frequency of formulas used (more than once) in the 162 trials

Table 7.1 Signs and symptoms of TCM patterns of disharmony of PMS

$\begin{array}{lll}\text { Table 7.2 Treatment for TCM patterns of disharmony } & 191\end{array}$

$\begin{array}{lll}\text { Table 8.1 Demographic Profiles } & 199\end{array}$

$\begin{array}{lll}\text { Table 8.2 Cigarette and alcohol consumption } & 201\end{array}$

$\begin{array}{lll}\text { Table 8.3 Happiness at work and family life } & 201\end{array}$

$\begin{array}{lll}\text { Table 8.4 Menstrual history } & 202\end{array}$

$\begin{array}{lll}\text { Table 8.5 Menstrual characteristics } & 204\end{array}$

$\begin{array}{lll}\text { Table 8.6 PMS experiences } & 205\end{array}$

Table 8.7 Comparisons of Group I and Group II on symptoms by 206 categorical clusters

$\begin{array}{lll}\text { Table } 8.8 & \text { Most common presenting symptoms } & 207\end{array}$

$\begin{array}{lll}\text { Table } 8.9 & \text { Psychological Characteristics } & 208\end{array}$

Table 8.10 The distribution of Chinese patterns in groups 210

$\begin{array}{lll}\text { Table 8.11 Tongue signs recorded at the first interview } & 211\end{array}$

$\begin{array}{lll}\text { Table 8.12 Pulses recorded at the first interview } & 212\end{array}$ 
Table 9.1 Mean differences and percentages of reduction between

baseline and month 3 of Group I (herb) and Group II (placebo)

Table 9.2 Significance of mean differences between time phases of Group II

Table 9.3 Time effects on all clinical assessments in Group II

Table 9.4 Mean differences of clinical assessment scores between

baseline, placebo and herbal treatment months in Group II

Table 9.5 Time and Measurement Effects between baseline and three months herbal treatment in Group I

Table 9.6 Effects of herbal treatment on symptom reports in Group I

Table 9.7 Comparisons between baseline and three months of herbal treatment on Physical symptoms, Psychological symptoms, Depression, Anxiety, Anger and Perceived Stress in Group I

Table 9.8 Time and Measures Effects and Measures by Time

Interactions between baseline and three months herbal treatment in Group II

Table 9.9 Significant differences over the four time periods (the last month of placebo treatment and the three months of herbal treatment) in Group II

Table 9.10 Comparisons between baseline and three months of herbal treatment period of Physical symptoms, Psychological symptoms, Depression, Anxiety, Anger and Perceived Stress in Group II

Table 9.11 Time and measures effects and measures by time interactions between baseline, herbal treatment month 3 and follow-up on physical and psychological symptoms of Group II

Table 9.12 Effects between baseline, herbal treatment month 3 and follow-up on physical and psychological symptoms of Group II

Table 9.13 Comparisons between baseline, herbal treatment month

3 and follow-up of Physical and Psychological symptoms in Group II

Table 9.14 Significant clinical changes after three months herbal treatment of Group I 
Table 9.15 Significant clinical changes after three months herbal treatment of Group II

Table 9.16 Clinically significant changes at follow-up of Group II

Table 10.1 Treatment effects on premenstrual symptoms collected from MSD and MDQ at baseline and the three herbal treatment months in Group I and Group II

Table 10.2 Comparison of baseline scores between women who completed all MSD diaries and those not

Table 10.3a Correlation between clients' satisfaction rating and

clinical assessment scores at the third month of experimental period

Table 10.3b Correlation between clients' satisfaction rating and

clinical assessment scores at the sixth month of experimental period

Table 10.4 Time, Measure and Pattern interactions between three herbal treatment month

Table 10.5a Treatment effects between TCM patterns at the three months of herbal treatment condition

Table 10.5b Scores differences of all measures between TCM patterns at the end of herbal treatment condition

Table 10.6 Comparison between baseline and after herbal treatment on Tongue signs

Table 10.7 Comparison between baseline and after herbal treatment on Pulses

Table 10.8 Tongue coating in TCM patterns before and after herbal treatment

Table 10.9 Tongue body in TCM patterns before and after herbal treatment

Table 10.10 Pulse symptoms in TCM patterns before and after herbal treatment 


\section{List of Figures}

Page

$\begin{array}{lll}\text { Figure 5.1 Flowchart of search results } & 147\end{array}$

Figure 5.2 Comparison between Chinese herbal medicine and 157 control in Full recovery from fixed-effects model

Figure 5.3 Comparison between Chinese herbal medicine and 158 control in Outstanding outcome from fixed-effects model

Figure 5.4 Comparison between Chinese herbal medicine and control in Improvement outcome from fixed-effects model

Figure 5.5 Comparison between Chinese herbal medicine and 160 control in No effect from fixed-effects model

Figure 7.1 Subject Pattern assignment flow diagram 1 174

$\begin{array}{lll}\text { Figure 7.2 } & \text { Subject Pattern assignment flow diagram 2 } & 174\end{array}$

$\begin{array}{lll}\text { Figure 7.3 Study Timeline } & 175\end{array}$

$\begin{array}{lll}\text { Figure 8.1 Summary diagram of participant flow } & 198\end{array}$

Figure 8.2 Distribution of TCM patterns 209

Figure 9.1 Effects of the baseline, three months herbal and 216 placebo treatment on Physical symptoms, Psychological symptoms, Depression, Anxiety, Anger and Perceived Stress

Figure 9.2 Treatment outcomes of Group II 219

Figure 9.3 Treatment outcomes comparing baseline and three months of herbal treatment in Group I

Figure 9.4 Treatment outcomes of pre-treatment and three months 232 herbal treatment in Group II

Figure 9.5 Mean scores at baseline, the third month of herbal 237 treatment and follow-up on physical and psychological symptoms (MDQ) in Group II 


\section{List of Appendices}

\begin{tabular}{|c|c|}
\hline Appendix A & $\begin{array}{l}\text { The Guidelines for Clinical Research in New TCM } \\
\text { Medicines for Pre-menstrual tension syndrome, Ministry of } \\
\text { Health, People's Republic of China }\end{array}$ \\
\hline Appendix B & $\begin{array}{l}\text { Professional Standards of Chinese Medicine, State } \\
\text { Administration of Traditional Chinese Medicine, People's } \\
\text { Republic China }\end{array}$ \\
\hline Appendix C & $\begin{array}{l}\text { Reference List of the } 124 \text { Chinese Clinical Trials between } \\
\text { 1996-2005 }\end{array}$ \\
\hline Appendix D & Randomisation Table \\
\hline Appendix E & Study Poster \\
\hline Appendix F & Study Flier \\
\hline Appendix G & Menstrual Distress questionnaire (MDQ) \\
\hline Appendix $\mathrm{H}$ & Menstrual Symptom Diary (MSD) \\
\hline Appendix I & Beck Depression Inventory (BDI) \\
\hline Appendix $\mathbf{J}$ & State Personality Inventory (STPI) \\
\hline Appendix K & Perceived Stress Scale (PSS) \\
\hline Appendix L & Demographic Questionnaire \\
\hline Appendix M & Information Statement for Participants \\
\hline Appendix $\mathrm{N}$ & Informed Consent Form \\
\hline
\end{tabular}


$\begin{array}{lll}1.1 & \text { Background } & 2\end{array}$

$\begin{array}{lll}1.2 & \text { Summary of the Study }\end{array}$ 


\subsection{Background}

Premenstrual syndrome (PMS) is a common disorder among women during their reproductive years that is characterised by a range of cyclical physical and/or mood symptoms experienced during the premenstrual period. PMS has many symptoms and an idiosyncratic nature. Premenstrual experiences, both benign and serious, can be expected to last until the menopause. PMS is reported to have a high prevalence rate in that up to one third of Australian reproductive-aged women have been reported to have severe PMS problems which impose enormous burdens on themselves and their families. Despite years of research, the exact cause and pathophysiology of PMS is still not completely understood (Kessel, 2000). There is still no widely effective and side effect free western medicine presently available to treat PMS. In China, descriptions of the specific symptoms relating to the premenstrual and menstrual phases, and discussions of their etiology, pathogenesis, diagnostic and treatment methods can be found in various Chinese medical texts, the earliest being from the Ming Dynasty (1368-1644).

Traditional Chinese medicine (TCM) is a medical system in China that has been used for thousands of years to prevent, diagnose, and treat diseases. It is based on a concept of balanced $Q i$ (vital energy) that is believed to flow throughout the body. $Q i$ is proposed to regulate a person's spiritual, emotional, mental, and physical balance and to be influenced by the opposing forces of yin and yang. A disorder is proposed to result from the flow of $Q i$ being disrupted 
and yin and yang becoming imbalanced. Among the components of TCM are herbal and nutritional therapy, restorative physical exercises, meditation, acupuncture, and remedial massage (MedicineNet.com). The etiology and pathogenesis of the signs and symptoms of PMS from a TCM perspective can be theorized as Liver Qi Stagnation, which is the proposed root cause, and its pathological changes that affect other systems and organs of the body (He $\&$ Wang, 1995) In this tradition, the application of holistic care and the use of natural herbs in TCM for PMS are well documented and supported by many centuries of clinical practice and experience (Deng 1999).

PMS usually includes both physical and emotional symptoms. Over 150 symptoms have been attributed to PMS by some researchers (Budeiri et al. 1994). Symptoms and discomfort level vary from one woman to another with each woman reporting her own unique syndrome (Berke et. al., 2001), which may vary from month to month and there may even be symptom-free months. While western medicine utilises symptomatic relief approaches, Chinese medicine employs a holistic treatment plan which covers both psychological and physical symptoms, and strives to address the hypothesised root cause of the syndrome

For some women, PMS may last until the menopause (Daugherty 1998), therefore any intervention should be safe for long-term use and free from any adverse side effects. Chinese herbal medicine has been used for thousands of years, and is accepted by Chinese medicine practitioners through clinical experience as being safe and effective. 
TCM provides the aetiology and pathogenesis of the symptoms of PMS. It uses Pattern Identification to diagnose the signs and symptoms of individual patients and to design a course treatment to address the entire pattern/s of disharmony. It follows that PMS, which has many symptoms and an idiosyncratic nature, is precisely an area for which Chinese medicine is most suitable. In view of these facts, it is clear that PMS is a condition for which Chinese medicine may be most suitable as an effective alternative treatment to western medicine. However, confidence in the treatment is largely based on practice that has not been properly evaluated scientifically. Although China has been advocating a more scientific approach to TCM since the 1950's, the awareness of PMS and knowledge of western research methods only became more common among Chinese researchers after Deng Xiaoping's "Open Door" policy in the late 70's. Pioneer studies such as Xu (1982) and Cheng and colleagues (1982) were carried out in the early 80's, and very few studies on PMS were randomized controlled trials (RCTs). The author conducted an electronic search for articles on clinical studies of PMS treatment using Chinese herbal medicine in Chinese TCM journals from 1996 to 2005 (see Chapter 5). Among the 124 clinical trials that had been recorded, 24 controlled studies were identified with only two being controlled and double blind trials. Qiao and colleagues (2002) examined the efficacy of herbal granules on Liver $Q i$ invasion (a sub-pattern of Liver $Q i$ stagnation) of PMS. They provided herbal granules and identical pills of other TCM herbal medicine for the control group. Li and colleagues examined the effect of herbal medicine on the depressed type of PMS, and used identical pre-packed TCM decoction (herbal 
solution). Both studies examined only one TCM pattern type in PMS. No rigorous clinical trial on the use of Chinese herbs to treat PMS which satisfies the western demands for hard, thoroughly researched evidence has so far been identified.

A true experimental study includes three properties i.e. randomisation, control and manipulation that would allow for other explanations of the phenomenon to be ruled out and thereby provide the strength of the design for testing cause-and-effect relationships (LoBiondo-Wood \& Haber 1990). A double-blinded design is generally considered appropriate to provide the most reliable findings from a clinical trial (Spilker 1996).

This study was undertaken with the above issues in mind. Investigation was therefore designed with a view to seek support by strong probability that Chinese herbal medicine may provide a valuable and effective alternative treatment to western medicine for PMS sufferers. For a robust consideration, a specially designed experimental study which is double-blinded, randomised, and placebo-controlled was considered necessary to scientifically evaluate the effectiveness of Chinese herbal medicine and its effects on PMS symptoms. The study was conducted by qualified Chinese herbal practitioners and overseen by PMS experts, and the effects of Chinese herbal granules, an inactive granular powder, were evaluated. The outcome was considered according to the level of relief in the occurrence and severity of both the psychological and physical symptoms. Data collection was based on widely used questionnaires that have a high internal consistency, and have been 
thoroughly tested in applied research of PMS and related disorders. The outcome also provided evidenced-based data to support the treatment of PMS with Chinese herbal medicines. Only through rigorous evaluation methods can the efficacy of Chinese medicine on PMS be demonstrated.

\subsection{Summary of the Study}

Sixty-five treatment seeking women with confirmed PMS were invited to join the study. On the basis of the information gathered, the researcher/practitioner made a diagnosis of the TCM pattern of disharmony of each participant. The woman was then grouped into one of the TCM pattern subgroups (Patten A: Liver $Q i$ stagnation, Pattern CA: Liver and Kidney Yin deficiency, Pattern H: Liver \& Spleen disharmony and Pattern D: Heart \& Spleen deficiency). In each of these TCM pattern subgroups, sequential allocation to Group I or Group II was carried out. Herbal medicine and placebo were provided sequentially to the subjects in both group I and II for a period of three months each. The participants in Group I received herbal medication for the first three months and placebo for the following three months. The participants in Group II received placebo for the first three months and herbal medication for the following three months.

There was marked improvement at the end of the first month of the herbal treatment, gradual and steady improvements continued throughout the next two months leading to a substantial reduction in symptoms on completion of the 
herbal treatment. The reduction in symptoms occurrence and severity of PMS was maintained at three month follow-up. Herbal treatment also achieved a better treatment outcome than the placebo treatment. Overall, the study provided positive support both statistically and clinically to the effectiveness of Chinese herbal medicine for PMS symptoms on a sample of Australian women. The effectiveness of the treatment was further reflected in the women's self reports of satisfaction with the treatment received. 
Chapter 2: Western Biomedical Perspective on Issues for Premenstrual Page Syndrome

$\begin{array}{llr}2.1 & \text { Issues of Definition }\end{array}$

$\begin{array}{lll}2.2 & \text { History of PMS } & 11\end{array}$

$\begin{array}{lll}2.3 & \text { Prevalence of PMS } & 15\end{array}$

$\begin{array}{lll}2.4 & \text { Theories of Aetiology } & 17\end{array}$

$\begin{array}{ll}2.5 & \text { Symptomatology in PMS }\end{array}$

2.5.1 PMS Symptoms in Chinese and Western Women 23

2.5.2 Functional Impairment from PMS Symptoms 24

$\begin{array}{lll}\text { 2.5.3 Classification of PMS Symptoms } & 27\end{array}$

$\begin{array}{ll}\text { 2.6 Diagnosis of PMS } & 30\end{array}$

2.6.1 Current Diagnostic Standards 31

$\begin{array}{lll}\text { 2.6.2 Summary } & 33\end{array}$

$\begin{array}{lll}2.7 & \text { Subtypes of PMS } & 34\end{array}$

$\begin{array}{lll}2.8 & \text { Overview of Treatment for PMS }\end{array}$

2.8.1 Non-pharmacologic Treatments of PMS 37

2.8.2 Pharmacological Interventions of PMS 43

2.8.3 Summary 46 


\subsection{Issues of Definition}

Premenstrual syndrome (PMS) was first described by the British physician, Dr Kathrina Dalton in 1964. She differentiated the condition from Frank's earlier (1931) description of premenstrual tension (PMT) by broadening the criteria to include the psychological changes that occur with the physical changes.

PMS covers a wide variety of cyclical symptoms which recur regularly during the premenstruum or in the early days of menstruation, with rapid and complete relief at the onset of full menstruation. Due to the wide range of signs and symptoms, severity, the relationship to menstruation, and the behavioural changes which women experience as PMS, researchers, physicians and psychologists have given different emphases to the definition of PMS.

Severino and Moline (1989) of America in their book refer to PMS as the cyclic occurrence of physical, behavioural or mood changes that appear to vary in severity during the late luteal phase of the cycle, that do not exist in the same form or severity during the mid or late follicular phase, and which disappear or return to their usual level during the full flow of menses.

Morse and Dennerstein (1988) of Australia defined PMS as a condition that "is characterised by negative moods, physical changes and alterations in cognitions and behaviours during the latter part of the menstrual cycle. Such symptoms 
diminish at the onset of menstruation or during the menstrual flow, and the post-menstrual (follicular) phase is usually one of calm and well-being".

Backstrom and Hammarback (1991) of Sweden define PMS as "a combination of mental and physical symptoms arising in the luteal phase of the menstrual cycle. The symptoms disappear after the onset of menstruation. During the rest of the follicular phase the patient is free from symptoms. The cyclical nature of the symptom variations is characteristic of the syndrome."

In this century, Freeman (2003) of America defines PMS as a generic term which includes a broad group of emotional, behavioural and physical symptoms that occur for several days before menses and subside following the menstrual period.

Most experts engaged in PMS research agree that timing, temporality of symptoms to cycle phase and severity are the key elements. The symptoms of PMS must occur in the late luteal phase, resolve within one or two days after the onset of menstrual bleeding and disappear completely by the mid-follicular phase (one week after the onset of menstruation). The symptoms must be recurrent, and severe enough to disrupt the women's daily life (Parker 1994). It is quite clear that PMS is a common condition and that women of the western world suffer similar symptoms. 


\subsection{History of PMS}

Although clinically significant, menstrual problems with mood and behavioral changes have in fact been recognised since ancient times (Endicott 2000), PMS only emerged as a distinct diagnosis and has been documented after an international consensus workshop in 1976 (Ugarriza et al.1998).

Medical practitioners and general laypersons only began to realize some seventy years ago that there are certain characteristic symptoms in particular reproductive-aged women which are associated with the premenstrual phase of the menstrual cycle (Severino and Moline 1989). No reports relating to premenstrual symptoms were found in a survey (Janiger et al. 1972) of historical and anthropological sources which was carried out in 1972, although several examples of cultural myths, taboos and superstitions associated with menstruation have been recorded. However, sporadic references with no distinction between premenstrual and menstrual complaints had occurred in historical writings (Richardson 1995). The earliest references to menstrual disorders can be found in the writings of Hippocrates (around 400BC), who described a pathological condition of uterine "gaseous" distension, explained as due to the accumulation of black bile in the uterus, which in seeking a channel of escape, was manifested in headache, vertigo, melancholia, black urine and vaginal discharge (Ricci 1950, cited in Richardson 1995). In the $19^{\text {th }}$ century, Prichard (1837) referred to the "nervous excitement: morbid dispositions of mind, a wayward and capricious temper, excitability in the feelings, moroseness in disposition, a proneness to quarrel with their dearest relatives, 
and sometimes a dejection of mind approaching to melancholia" experienced by some females at the time of menstruation and termed as "dysmenorrhoeal affections" (cited in Richardson 1995).

However, Stolberg (2000) argued that premenstrual somatic, emotional and behavioural disorder can be traced back over hundreds of years. Premenstrual suffering was frequently described as a very common complaint by physicians and women alike from the late Renaissance. For example, Jacques Dubois (1478-1555) stated that many women "develop a strangulation from the uterus" at the approach of menstruation (cited in Stolberg 2000).

The contemporary hallmark study of premenstrual problems took place in 1931 when Frank coined the term "premenstrual tension" to describe the collection of predominantly somatic complaints brought to him by female patients (Endicott 2000). Later, Greene and Dalton (1953) expanded the work, and found the term "premenstrual tension" unsatisfactory as tension is only one of the many components of the syndrome. They proposed the term "premenstrual syndrome" which referred to both somatic and mood problems. Abraham (1980a,b) recognized that nervous tension is one of the most common symptoms of the syndrome, so he chose to use premenstrual tension syndrome (PMTS). After 1953, premenstrual syndrome, premenstrual tension syndrome and premenstrual tension have been used synonymously (Johnson 1987) until the consensus workshop thirty years ago where among many issues precise definitional criteria were adopted and the term PMS became widely used. However, premenstrual tension syndrome is a term that is more often used by 
authors outside English speaking countries, such as Zoldan and colleagues (2004) of Brazil, Oguchi (2003) of Japan, Legros and colleagues (1999) of France, López-Rosales and colleagues (1996) of Mexico, and Andersch and colleagues (1990) of Sweden.

In the early 1980's, PMS received worldwide attention and became a major issue for both medical professionals and lay communities, following an international consensus workshop in 1983 conducted by the National Institute of Mental Health (NIMH) of America. Further, a highly publicized legal case in the UK in 1987 accepted severe PMS as legitimate medical evidence for mitigation and as an adequate basis for a plea of manslaughter with diminished responsibility for behavioural aberrations in two murder cases, each involving a British woman (Johnson 1987). Debates and disagreement on whether premenstrual syndrome is a true medical condition have been continuing since then to the present time. Some medical practitioners and feminists have questioned whether the relevant symptoms amount to a pathological condition which warrants and legitimises medical intervention and treatments (Richardson 1995).

Although the symptoms associated with the premenstrual period have been acknowledged in contemporary times by medical professionals and general society for more than 70 years, PMS has only been defined as an accepted class in medical and lay disease classification systems in western industrial cultures in the past four decades. The ninth revision of the International Statistical Classification of Diseases and Related Health Problems (ICD-9) of the World 
Health Organisation (WHO) in 1979 classified premenstrual tension syndrome under "Noninflammatory diseases of the female genital tract". The American Psychiatric Association (APA) in 1987 included the severe dysphoric type premenstrual syndrome: Late Luteal Phase Dysphoric Disorder (LLPDD), as a proposed diagnostic category in the appendix of the revised third edition of the Diagnostic and Statistical Manual of Mental Disorders (DSM-III-R).

To date, physicians and psychiatrists still include PMS in their classification manuals. The premenstrual tension syndrome is classified as a gynaecological disease under the same category: "Non-inflammatory disorders of the female genital tract" in the 2006 version of the tenth revision of the International Statistical Classification of Diseases and Related Health Problems (ICD-10), unchanged from the ICD-9. Conversely, the fourth edition of the Diagnostic and Statistical Manual of Mental Disorders (DSM-IV) published in 1994, changed the name of the condition from LLPDD to Premenstrual Dysphoric Disorder (PMDD) with criteria that were almost identical to those of LLPDD except for the inclusion of one additional item. The APA included PMDD in Appendix B: Criteria sets and axes provided for further study in DSM-IV and the fourth edition - text revision of the Diagnostic and Statistical Manual of Mental Disorders (DSM-IV-TR) in 2000. They classified PMDD as an example of $a$ depressive disorder not otherwise specified to distinguish from the 'common' or generalised premenstrual syndrome. The criteria for diagnosis of the disorder in DSM-IV-TR are almost identical to those in the previous DSM-IV. 
In summary, PMS was only recognised as a predominant somatic disorder when Frank first described the condition in 1931. Greene and Dalton (1953) later referred to the condition as comprising both somatic and mood problems. The condition called PMS has been questioned as to whether it is a true medical condition. To date, while physicians classify it as a physical gynaecologocal disorder, psychiatrists distinguish premenstrual problems due to severe dysphoric mood changes as a "mental disorder" from the common premenstrual syndrome without such severe moods. This differentiation between common and severe dysphoric premenstrual problems should assist medical professionals in deciding treatment options e.g. the use of antidepressants and other psychotropic medications as treatments for sufferers of PMS.

\subsection{Prevalence of PMS}

The prevalence rate of PMS has varied in studies (Andersch et al. 1986; Woods et al 1982a \& 1986; Halbreich 2003; Zhao et al. 1998; Ramcharan 1992; Deuster et al. 1999; Freeman et al. 1995a; Sternfeld et al.2002; Tatarchuk et al. 1999). Depending on the methods of assessment and the samples of women selected, studies in various countries indicate that up to $95 \%$ of women of reproductive age have reported a wide range of premenstrual changes of varying degrees of severity. A mail survey carried out twenty years ago (Andersch et al. 1986) of premenstrual symptoms on 1083 Swedish women aged 18-24 years indicated that $92 \%$ of them had experienced some symptoms of PMS, and that 2-3\% reported these as severe. However, the most commonly 
reported prevalence rate of PMS is around 30\%-40\% (Woods et al 1982a \& 1986; de la Gandara Martin and de Diego Herrero 1996; Campbell et al.1997; Wittchen et al. 2002). Halbreich (2003) has pointed out that PMS studies repeatedly cite 3-8 \% of women as having PMDD. Consistent with the above findings in western countries, a Chinese study of 454 reproductive-aged women in Beijing, China (Zhao et al. 1998) indicated that the prevalence of PMS was $30.4 \%$, of which $61.65 \%$ was mild, $34.1 \%$ moderate, and only $4.3 \%$ was severe. These figures from both the Asian and western worlds provide very similar estimates.

However, American gynecologists and psychiatrists have different opinions on this prevalence issue. The American College of Obstetricians and Gynecologists (ACOG) suggests that only 5-10\% of women experience severe symptoms that affect their abilities to function at home, in the work place or in their relationships with others (ACOG Practice Bulletin 2000). Nevertheless, the American Psychiatric Association (1994) estimated a prevalence of minor premenstrual changes in psychological functioning in approximately $75 \%$ of women, with approximately $20 \%-50 \%$ reporting symptoms characteristic of PMS, and 3\%-5\% meeting the criteria for the severe dysphoric form of PMS i.e. PMDD.

The finding on the time course of the disorder has also varied. Most studies e.g. Ramcharan (1992) of Canada, Freeman and colleagues (1995a) and Sternfeld and colleagues (2002) of America have acknowledged that symptom severity in PMS declines as women approach menopause. Also in a population-based study 
of all women aged 18-44 in the State of Virginia, USA (Deuster et al. 1999), the 35-44 year age group was the least likely to experience PMS (4.5\%) compared to the 18-24 year group (8.7\%) and the 25-34 year group (10.4\%). Conversely, another study in Ukraine (Tatarchuk et al. 1999) found that the frequency of severe forms of PMS increased with age, the prevalence of the 'edematous' form (water retention)of PMS was reported in the group aged 18-29 years, the 'neuropsychopathic' form in the group aged 30-35 years and the 'crisis' form in the group aged 36-45 years. However, unless further scientific evidence is available, this result should be viewed with caution as it deviates significantly from the findings of most studies.

The variations in the prevalence in PMS reflect the different approaches to the use of diagnostic standards, symptom recognition, and/or methods of measurement. Working towards a set of universally agreed criteria for the diagnosis of the disorder is therefore urgently required.

\subsection{Theories of Aetiology}

After more than 70 years of study, there seems to be no exact aetiology and commonly accepted causation of PMS. Aetiologic research has been pursued under hormonal, psychosocial, nutritional, biochemical, and/or metabolic frameworks. 
Pioneer PMS researchers, such as Frank (1931) and Israel (1938), implied that some imbalance existed between the ovarian hormones that provided the underlying explanation for the disorder. Frank (1931) hypothesised that premenstrual tension was caused by an excessive accumulation of oestrogen due to renal dysfunction with a high renal threshold of hormones causing retention of oestrogen in the body. Greene and Dalton (1953) proposed that PMS was the result of water retention which was caused by an abnormal elevation of the ratio between the circulating levels of oestrogen and progesterone. Some researchers also implicated the cause of PMS to fluctuating levels of deficiency, excess and/or imbalance of gonadal hormones. However, research studies have so far failed to establish any specific relationships between the concentrations of these hormones and PMS symptoms (Richardson 1995), and serum ovarian steroid concentrations have repeatedly been shown to be normal in many PMS studies of women with normal reproductive function (Ismail and O’Brien 2005).

Several researchers (e.g. Morse and Dennerstein 1988; Gotts and colleagues 1995; Richardson 1995) pointed out that the experiences of PMS sufferers are primarily psychological in nature. These symptoms vary widely in their incidence across different cultural groups. Considerable evidence indicates that women's experiences of premenstrual symptoms may have more to do with cultural or psychological factors than with biological ones (Richardson 1995).

Abraham (1983) linked the presence of PMS signs and symptoms to nutritional deficiencies and excesses. Vitamin B and magnesium deficiencies have been 
proposed as contributing factors to the emotional symptoms of PMS of anxiety and depression; reduced vitamin B6, vitamin E and magnesium are regarded as contributing factors to fluid retention symptoms; reduced vitamin B, vitamin C, zinc and magnesium are proposed as contributing factors to carbohydrate craving. However, Mira and colleagues (1988) reported that no evidence was found to support these hypotheses in their study.

Excessive consumption of refined sugar, fat, caffeine (tea, coffee, or chocolate), cigarettes and alcohol are thought to trigger PMS signs and symptoms in some women. Studies carried out in America (Perry et al. 2004; Rossignol \& Bonnlander 1990) and in a mailed survey of 14,779 women in Australia (Mishra 2000) suggest that higher alcohol consumption, caffeine ingestion and smoking have been associated with increased premenstrual symptoms, but whether as cause or effect remains unclear. The outcome of another Australian study on overweight women with PMS (Cross et al. 2001) found that changes of food intake occurring in the menstrual cycle in PMS sufferers also indicate a significantly greater intake premenstrually $(\mathrm{p}<.001)$ of energy producing foods from all macronutrients found in cereals, cakes, desserts and high-sugar foods. Dye and Blundell's review of 30 studies (1997), revealed that 25 studies reported significantly higher energy intake in the luteal phase compared with the follicular phase. These findings suggest that the increase in high-sugar consumption could be a contributing factor potentially, or they are just a result of PMS symptoms. The key issue here is the direction of causation. That is, whether the increased energy producing foods consumed produce the symptoms, or whether the symptoms experienced motivate women to consume 
greater amounts of energy producing foods and stimulants (e.g. caffeine in coffee and chocolate).

Women who are PMS sufferers show decreased glucose levels in glucose tolerance tests carried out in the luteal phase (Reid et al. 1986). This depletion may give rise to the hypoglycaemic signs and symptoms of PMS e.g. sugar craving, irritability, tiredness or weakness, dizziness, poor memory or concentration, heart palpitations, depression or mood swings, anxiety or nervousness. Brayshaws (1986) proposed that there may be a possible link between thyroid dysfunction and PMS, as both conditions produce similar symptoms. Other current studies (Nikolai et al. 1990; Korzekwa et al. 1996) reported that either there is no significant thyroid disease in PMS or hypothyroidism is not the cause. Schmidt and colleagues (1993) of the National Institute of Mental Health (NIMH) reported that thyroid dysfunction is found in a small group of women with PMS, but PMS should not be viewed as a masked hypothyroidism.

None of the above models has given rise to treatment for PMS that has proved to be consistently effective in appropriately controlled clinical trials (Parry 1994). Findings in recent years constantly suggest that PMS is multifactorial, with the involvement of hormones, neurotransmitters, neurosteroids, nutrition and psychosocial factors (Steiner, 2000). Several biologic mechanisms that underlie menstrually related symptoms have been proposed. The interactions of fluctuating levels of gonadal hormones or their metabolites with neurotransmitter systems or receptor imbalances in the brain may contribute to 
menstrually related symptoms (Rubinow and Schmidt 1995; Ismail \& O’Brien 2005). Of all the neurotransmitters, evidence suggests that serotonin (5-HT) plays an important role in the pathogenesis of PMS (Lepage and Steiner 1991; Rapkin 1992; Halbreich 1999). The cumulative effects of oestrogen act as an agonist on serotonergic (5-HT) function by increasing the number of serotonin receptors, serotonin postsynaptic responsiveness, neurotransmitter transport and uptake (Halbreich et al. 1995; Matsumoto et al.1985; McEwen et al. 1997). Taylor and colleagues (1984) also indicated that a reduced platelet uptake of serotonin occurs during the week before menstruation as compared to the postmenstrual phase in women with premenstrual syndrome, and that those women demonstrate a lowered level of platelet serotonin content (Ashby et al. 1988). Genetic factors also seem to be involved, as studies indicate that the concordance rate of PMS is higher in monozygotic twins than in dizygotic twins (Dalton et al. 1987; Condon 1993). Even so, after considerable study and research, no exact hormonal nor neurochemical dysfunction has been identified that can explain the disturbances (Dickerson et al. 2003).

Halbreich (2003) proposed that the etiology of premenstrual symptoms involves two diversified stages. The first is a hypothesized hypersensitivity of individual woman to changes in gonadal hormones, where external or internal stressors during the luteal phase contribute to CNS dysregulation and impaired adaptation that give rise to premenstrual symptoms. The second stage of genetic vulnerability involves phenotype predisposition. Different women may be predisposed to phenomena resulting from abnormalities in different CNS systems. These hypotheses remain to be confirmed in well designed studies. 


\subsection{Symptomatology in PMS}

Up to 300 different premenstrual symptoms have been reported by women who seek help for PMS (Halbreich et al. 1982), with each woman reporting her own unique set (Berke et al. 2001). For example Budeiri and colleagues (1994) in their literature review found that a total of 199 PMS symptoms were reported in 350 clinical trials. The most common symptoms from the studies include a short list of: abdominal bloating, painful or swollen breasts, irritability, mood swings, depression and/or tension, anxiety, weight gain, headache, skin disorders, cramps, backache, fatigue and particular food cravings (Greene \& Dalton 1953; Woods et al. 1982b; Ramcharan et al. 1992; Gotts et al. 1995; Zhao et al. 1998, Hylan et al.1999; Walsh \& Polus 1999; Freeman 2003).

For the diagnosis of the severe dysphoric form of PMS (PMDD), the APA (1994) requires that at least five of the eleven specific PMS symptoms are present most of the time during the last week of the luteal phase. These PMDD symptoms include markedly depressed mood, marked anxiety/tension, marked affective lability, marked anger/irritability, decreased interest in daily life, concentration difficulties, fatigue, marked appetite changes/food craving, insomnia/hypersomnia, feeling out of control, and physical symptoms which are evaluated as a single entity (further details will be discussed in Subdivision 2.6.1). Clearly, these core symptoms are psychological and emotional in character indicating that the profound psycho-emotional distress reported by 
women of PMDD is a mental disorder and is more severe than the general group of PMS symptoms.

\subsubsection{PMS Symptoms in Chinese and Western Women}

Symptoms and discomfort levels vary from woman to woman, but PMS is reported by women from all cultures and from different socio-economic and ethnic backgrounds (ACOG Practice Bulletin 2000).

A study by Hylan and colleagues (1999) in the United States, the United Kingdom and France revealed that irritability/anger, fatigue, and physical swelling/bloating or weight gain were among the most common reported symptoms. Results were generally consistent across the three countries. Sveinsdottir and Marteinsdottir (1991) reported that Icelandic women experience premenstrual changes as do their peers in other countries, and the most common symptoms are bloating, mood swings, irritability, abdominal discomfort-pain, and intolerance-impatience. Gotts and colleagues (1995) conducted a study among ninety-eight women seeking PMS treatment in Melbourne, Australia. Thirty-two different psychological or physical symptoms were reported. The most frequently reported symptoms were irritability and depression, and these negative affective symptoms comprised at least half of the frequency of the total symptoms experienced. Walsh and Polus (1999) also reported the distribution and frequency of major PMS symptoms in their study $(n=45)$ of Australian women. The major symptoms were irritability and/or 
anxiety, moodiness and/or anger, depression, bloating, headache, breast tenderness, fatigue, low-back pain and abdominal cramps.

Zhao and colleagues (1998) reported that the order of frequency of the PMS symptoms of women in Beijing, China was irritability, depression, anxiety, diarrhoea, lack of concentration and hypersomnia. Holroyd and colleagues (1998) explored the existence and experience of PMS within a Hong Kong Chinese cultural setting and found that cramps, fatigue and mood swings constituted the most frequently reported items. Another investigation (Kuo et al. 1997) of premenstrual discomfort among Chinese nurses in Taiwan indicated that the most bothersome premenstrual complaints appeared in the sequence as emotional instability, abdominal discomfort, pain, and edema. The frequency and order of these common symptoms in PMS in different studies of various countries are summarised in Table 2.1.

Thus, these findings indicate a considerable similarity of reported symptoms and the dominance of negative moods in both western and Chinese PMS sufferers.

\subsubsection{Functional Impairment from PMS Symptoms}

Several studies (Campbell, 1997; Hylan et al.1999; Pearlstein et al. 2000; Robinson and Swindle, 2000; Halbreich 2003) indicate that PMS sufferers report functional impairment in many areas of their lives during the last week 
of their menstrual cycle. The greatest impairment is frequently in personal relationships due to emotional instability characterised by irritability and impatience or sadness and depression, and in compromised work capacities arising from tension, fatigue, boating and disturbed sleep. Campbell and colleagues (1997) reported in their survey that PMS had caused difficulties to Australian sufferers in their family/friends relationships, work, social activity, and sexual relationships. Hylan and colleagues (1999), in a survey in the United States, United Kingdom and France, revealed that impairment due to PMS tends to be highest at home, in family/ spousal relations, followed by social, school, and occupational situations. In America, Robinson and Swindle (2000) revealed that the PMS experiences in their 1022 surveys are reported to frequently or always interfere with the women's functioning in most life domains i.e. work/school, household activities, family relationship and social life. The indications of changed psychosocial functioning in PMDD women appear to be similar to those women reporting major depression during the luteal phase (Pearlstein et al 2000). Halbreich (2003) reported that women with PMDD suffer impairment in their marital and parental relationships, social adjustment with a decreased perceived quality of life during the late luteal phase of the menstrual cycle. Thus the findings from studies carried out in different continents of the world reveal considerable similarities in symptom reports. These common experiences reveal the global nature of premenstrual adverse experiences in women of reproductive age. 
Table 2.1 The order and frequency of the most common PMS symptoms in different countries

\begin{tabular}{|c|c|c|c|c|c|c|c|c|c|}
\hline & USA $^{*}$ & $\mathrm{UK}^{*}$ & France $^{*}$ & Iceland $^{\diamond}$ & Australia & Australia $^{\mathbf{}}$ & Beijing $^{\circ}$ & Hong $=$ & Taiwan" \\
\hline & & & & & & & China & Kong & \\
\hline irritability/anger & 2 & 2 & 2 & 3 & 1 & 1 & 1 & & \\
\hline anxiety/tension & 6 & 6 & 5 & & 7 & 2 & 3 & & \\
\hline depression, & 8 & 5 & 6 & & 2 & 2 & 2 & & \\
\hline mood swings & & & & 2 & & & & 3 & 1 \\
\hline crying & 6 & 6 & 7 & & & & & & \\
\hline aggression & & & & 4 & 4 & & & & \\
\hline lack of concentration & & & & & & & 5 & & \\
\hline physical swelling & 1 & 1 & 1 & 1 & 6 & 3 & & & 3 \\
\hline \multicolumn{10}{|l|}{ (bloating) / weight gain } \\
\hline abdominal cramps & & & & 4 & & & & 1 & 2 \\
\hline fatigue & 3 & 3 & 3 & & 5 & 5 & & 2 & \\
\hline change in appetite & 4 & 4 & & & & & & & \\
\hline breast tenderness & 5 & 7 & 4 & & 3 & 4 & & & \\
\hline headache/ other pain & 7 & 8 & 5 & & 4 & 4 & & & \\
\hline insomnia / hypersomnia & & & 8 & & & & 6 & & \\
\hline diarrhoea & & & & & & & 4 & & \\
\hline
\end{tabular}

\begin{tabular}{|c|c|}
\hline${ }^{*}$ Hylan et al. 1999 & ${ }^{\circledR}$ Sveinsdottir \& Marteinsdottir 1991 \\
\hline${ }^{\star}$ Gotts et al. 1995 & rWalsh \& Polus 1999 \\
\hline${ }^{\circ}$ Zhao et al. 1998 & =Holroyd et al. 1998 \\
\hline
\end{tabular}




\subsubsection{Classification of PMS Symptoms}

To facilitate diagnosis, treatment, and/or research, different classification methods have been developed by subdividing the symptoms of the disorder into categories of related experiences.

Moos' taxonomy (1968), selected 47 common peri-menstrual symptoms, and by factor analysis clustered these into eight sub-scales that comprise the Menstrual Distress Questionnaire (MDQ) the most widely used symptom checklist for nearly forty years. The subscales are Pain, Water Retention, Autonomic Reaction, Negative Affect, Impaired Concentration, Behavioural Change, Arousal (positive) and Control. The first six clusters of symptoms measure negative perception; the seventh cluster measures positive perception and the eighth cluster contains symptoms that are infrequently reported (see Chapter 4 for further details). The purpose of the sub-grouping of symptoms is that it can be used for comparing self-reported estimates of the prevalence and severity of symptoms which allow the analysis of their relationships with psychological and/or biochemical factors of different types of PMS. The symptoms in each subscale are shown below in Table 2.2. 
Table 2.2 MDQ Subscales (Moo's 1968)

\begin{tabular}{|c|c|}
\hline Pain & Concentration \\
\hline Muscle stiffness & Insomnia \\
\hline Headache & Forgetfulness \\
\hline Cramps & Confusion \\
\hline Backaches & Lowered judgment \\
\hline Fatigue & Difficulty concentrating \\
\hline \multirow[t]{3}{*}{ General aches and pains } & Distractible \\
\hline & Accidents \\
\hline & Lowered motor coordination \\
\hline Water Retention & Behavioral Change \\
\hline Weight gain & Lowered school or work performance \\
\hline Skin disorders & Take naps; stay in bed \\
\hline Painful breasts & Stay at home \\
\hline \multirow[t]{2}{*}{ Swelling } & Avoid social activities \\
\hline & Decreased efficiency \\
\hline$\underline{\text { Autonomic Reaction }}$ & $\underline{\text { Negative Affect }}$ \\
\hline Dizziness, faintness & Crying loneliness \\
\hline Cold sweats & Anxiety \\
\hline Nausea, vomiting & Restlessness \\
\hline \multirow[t]{4}{*}{ Hot flashes } & Irritability \\
\hline & Mood swings \\
\hline & Depression \\
\hline & Tension \\
\hline$\underline{\text { Arousal }}$ & $\underline{\text { Control }}$ \\
\hline Affectionate & Feeling of suffocation \\
\hline Orderliness & Chest pains \\
\hline Excitement & Ringing in the ears \\
\hline Feeling of well-being & Heart pounding \\
\hline \multirow[t]{2}{*}{ Bursts of energy, activity } & Numbness, tingling \\
\hline & Blind spots, fuzzy vision \\
\hline
\end{tabular}


Abraham (1980a,b) developed a Menstrual Symptom Diary and divided 19 menstrual symptoms into four subgroups labelled A, C, D, and H (Table 2.3). Symptoms associated with PMT-A are largely affective, PMT-H refers to water and salt retention, PMT-C concerns carbohydrate craving, and PMT-D refers to depressive mood. Abraham (1983) attempted to assign nutritional causative factors to each of these symptom subgroups and proposed the application of therapy to rebalance the presumed deficiency.

Table 2.3 Subgroups in Abraham's Menstrual Symptom Diary

\begin{tabular}{lll}
\hline Type & Nutritional deficiency & PMS symptoms \\
\hline PMT-A & Vitamin B, Magnesium & anxiety, irritability, mood swings, nervous tension \\
PMT-H & Vit B6,Vit E, Magnesium & fluid retention, weight gain, swollen of extremities, breast \\
& & tenderness, abdominal bloating \\
PMT-C & Magnesium, vitB, vitC, & cravings, increase appetite, headache, fatigue, dizziness, \\
& Zinc & fainting, palpitation \\
PMT-D & Vitamin B, Magnesium & depression, crying, forgetfulness, confusion, insomnia \\
\hline
\end{tabular}

Gotts and colleagues (1995) reclassified 32 peri-menstrual symptoms reported by the women in their study $(n=92)$ into three categories of psychological symptoms and two categories of physical symptoms. Their aims were to examine the symptom heterogeneity and symptom severity in a group of treatment seeking women reporting PMS. The three psychological symptom categories were affective, behavioral expression, and cognitive dysfunction, 
and the two physical symptom categories were pain-related and non-pain-related. From this reclassification, they found that psychological affective symptoms were the most prevalent and serious category, and, that 72 out of the 98 subjects reported a psychological problem as their first most severe concern.

\subsection{Diagnosis of PMS}

The diagnosis of PMS is still fragmented and is still far from a widely accepted biomedical and/or biopsychosocial model of diagnostic criteria (Halbreich 2004). Essential characteristics for a PMS diagnosis require details of the timing (onset and offset) and the perceived severity of the symptoms, the degree of disruption in the women's functioning, and the differentiation of the disorder from other physical or psychiatric conditions (Freeman 2003). Because of the large number of symptoms associated with PMS and the absence of a specific diagnostic laboratory test or other diagnostic tool to confirm a diagnosis (Frye \& Silverman 2000), assessment of PMS therefore depends on what is reported by the patient (O'Brien 1987). The character of the symptoms may be less important than their timing and severity (Ismail and O’Brien 2005). 


\subsubsection{Current Diagnostic Standards}

Premenstrual syndrome was included in the ICD-9 and ICD-10 of the WHO. The diagnosis requires that symptoms such as tension, migraine or any other molimen must occur during the premenstrual phase of between 1 to 7 days before menstruation and remit following menses. However, the ICD-10 does not specify any other criteria such as severity, degree of symptom change or impairment, differential diagnosis or exclusion criteria. The ICD-10 diagnosis focuses only on the two main aspects of PMS, i.e. the timing and cyclic relationship with menstruation. It does not require a minimum number of symptoms nor degree of functional impairment. According to Halbreich (2004), it is the closest set of criteria yet to emerge that may attract universal acceptance.

According to the ACOG (2000), a diagnosis of PMS should include: at least one affective or somatic symptom; other symptoms consistent with the classification of defined PMS; restriction of these symptoms to the 5 days before menses at each of three prior menstrual cycles, with relief occurring within 4 days of the onset of menses; impairment at mild to moderate or severe levels in some facets of the woman's usual life; and the exclusion of other disorders (e.g. psychiatric illness). The information should be based on prospectively recorded diaries kept by the women, preferably every day for approximately eight to ten consecutive days for two to three consecutive months. These ACOG criteria acknowledge the timing and the nature of symptoms and the level of impairment, and require prospective confirmation of retrospective reports. It considers the individual 
diversity of symptoms and the requirement of at least one symptom, which avoids the constraint of a threshold of a specific number of symptoms.

In 1983, the National Institute of Mental Health (NIMH) proposed that the diagnosis of PMS was dependent on the occurrence of an increase in symptom severity of at least $30 \%$ in the six days before menstruation as compared with the days after menses. Documentation of these changes is required to be recorded in a daily symptom diary for at least two consecutive cycles (Parker 1994). The quantitative suggestion of at least a $30 \%$ change in perceived severity of the symptoms between premenstrual and post-menstrual phases, together with the charting of daily symptoms across two consecutive months led to the completion of diagnosis of PMS that differentiates it from the diagnostic criteria of other conditions, such as mood disorders, anxiety disorder, dysthymic disorder or personality disorder.

The most detailed diagnostic criteria of a subtype of PMS include the criteria for PMDD according to the DSM-IV-TR of the American Psychiatric Association (APA 2000). This diagnosis requires women sufferers (or someone with whom they live) to chart their symptoms daily for two complete cycles by prospective daily charting, and their chief complaints must include one of the four core symptoms (irritability, tension, dysphoria, and lability of mood) and at least five of eleven total symptoms. The symptoms should be present during most menstrual cycles in the past year; should demonstrate clear worsening during the last week of the luteal phase and should remit within a few days after the onset of the menstrual phase; should have interfered with social or 
occupational roles; and should not be an exacerbation of another chronic disorder. Despite being the most detailed diagnostic criteria of a subtype of PMS, there are no guidelines in the criteria for quantifying the measures on either the frequency of the symptoms, or the degree of the symptom severity or functional impairment.

There are variations between the current diagnostic categories of PMS. The current DSM-IV-TR requires at least five specified symptoms for a diagnosis of PMDD to be made, while the ICD-10 requires only one distressing symptom for a diagnosis of PMS to be made. The DSM-IV-TR criteria require a temporal criterion where symptoms must occur only or mainly during the week before menses, whilst the ACOG criteria require them to occur within 5 days before menses. However, both do not specify for how many days the symptoms should exist.

\subsubsection{Summary}

A universally accepted diagnostic entity is critically important for distinguishing between PMS and other disorders, for determining the prevalence of the disorder, for unifying disease coding, for further research, for regulatory purposes and most importantly, for determining suitable treatment. Diagnostic criteria for PMS must recognize the broad range of symptoms, the temporal pattern of the symptoms and the critical issue of symptom severity. A diagnosis of PMS should consist of the determination of the timing of the symptoms in 
relation to various phases of the menstrual cycle, the stipulation of significant changes between post-menstrual and premenstrual symptom severity, careful reports of impairment of functioning, and diagnostic differentiation to distinguish PMS from other medical and psychiatric conditions. To date, there has been no hormone or laboratory test which can provide an unequivocal PMS diagnosis and the current diagnostic standard requires confirmation of subjective symptom reports by prospective daily diaries.

\subsection{Subtypes of PMS}

Since the classification of diseases may contribute to specific treatments for each subtype, some researchers have attempted to identify subtypes of PMS by grouping women's experiences according to different clusters of symptoms or symptom severity.

The pioneer investigator of premenstrual problems, Frank (1931), observed that there were three types of women who presented to him complaining of premenstrual tension. The first type of women was those handicapped by premenstrual disturbances of a multiplex nature. The second group was those whose complaints were of sufficient gravity to require rest in bed for one or two days, and the last type consisted of those in whom severe systemic disorders manifested themselves, predominantly during the premenstrual period. Dalton $(1964,1984)$ described three categories of premenstrual disorder: premenstrual tension, a condition of psychological symptoms only; 
premenstrual syndrome (so called 'true' PMS) which refers to a combination of physical and psychological symptoms; and menstrual distress syndrome (MDS) which applies to patterns with similar symptoms present at a moderate level throughout the cycle, which worsen premenstrually.

In 1983, Abraham attempted to assign nutritional aetiological factors for each of four symptom subgroups, based on hypothesized nutritional deficits, in order to guide decisions about different nutritional supplement for the syndrome. The four subgroups are:-

- $\quad$ PMT-A, which includes affective symptoms of anxiety, irritability, nervous tension and behaviour patterns detrimental to self, family and society. The proposed cause is deficiency of vitamin B and magnesium.

- $\quad$ PMT-H, where water retention is the major problem. It is associated with water and salt retention, abdominal bloating, mastalgia and weight gain. The proposed cause is deficiency of vitamin $\mathrm{B}_{6}$ and $\mathrm{E}$, and magnesium.

- $\quad$ PMT-C, is characterised by craving for sweets, increased appetite and indulgence in eating refined sugar followed by palpitation, fatigue, fainting spells, headache and 'shakes'. The proposed cause is deficiency of vitamin B and $\mathrm{E}$, magnesium and zinc.

- $\quad$ PMT-D, the symptoms are depression, withdrawal, insomnia, forgetfulness and confusion, and the proposed cause is also deficiency of vitamin B and magnesium.

However, it is common for women to experience more than one of the subtypes in any month. Also, some of the nutritional deficiency factors, e.g. magnesium, 
vitamin $\mathrm{B}$, appear in all or more than one subtype thus rendering the classification somewhat confusing. Therefore, most recent treatment guidelines of PMS such as the ACOG Practice Bulletin (2000) recommend magnesium, calcium, and vitamin E to all PMS sufferers.

In the last 20 years, Late Luteal Phase Dysphoric Disorder (LLPDD) and Premenstrual Dysphoric disorder (PMDD) have appeared in the appendix of the Diagnostic and Statistical Manual of Mental Disorders (APA 1987, 1994). Both refer to women with premenstrual complaints who suffer primarily from serious affective symptoms which may be considered as the most severe forms of PMS (Taylor 1994). This subtype of severe form PMS i.e. PMDD, which is classified under the domain of psychiatric disorders allows the prescription of antidepressant drugs (SSRIs) for the treatment of the disorder.

In summary, the grouping of the above subtypes occurred in two stages. In the early stage, pioneer researchers of premenstrual problems grouped the PMS sufferers into subtypes according to symptom severity in order to confirm/grade the disorder. The more recently proposed subtypes were determined according to different clusters of symptoms. In each case, different subtypes call for differential treatment modalities. 


\subsection{Overview of Treatment for PMS}

After more than seventy years of targeted symptomatic and generalised treatments, there is still no complete cure for the disorder. Treatment goals for PMS are to reduce various symptoms, improve the social and occupational functioning of the sufferers, and minimize any adverse effects of the treatment (Bhatia \& Bhatia 2002). Therapeutic interventions for the disorder range from non-pharmacological management to psychotropic medications and hormonal therapy, or surgical procedures for the extreme severe cases (Steiner 1997). This last resort which may give rise to many significant risks in fact returns to Frank's (1931) original proposal of ovarian ablation to achieve relief.

\subsubsection{Non-pharmacologic Treatments of PMS}

Non-pharmacologic therapies include psychological therapy, aerobic exercise, nutritional supplements and dietary adjustments. These approaches may be attempted before prescribing medication, or after drug treatments, or simultaneously with pharmacological agents.

Psychological therapy may be an option for women who do not wish to take medications or where the dominant symptoms are psychological and behavioural in nature. Multiple trials (Kirkby, 1994; Christensen and Oei, 1995; Blake et al., 1998; Goodale et al., 1990; Morse et al., 1991; Hunter et al. 2002) have applied cognitive behavioural therapy and relaxation therapy to reduce the 
negative moods of PMS. A cognitive approach may help women to control the emotion, cognitive, and somatic effects of premenstrual syndrome through the acquisition of self-control, self-direction, and enhanced discomfort tolerance (Morse et al. 1991). In one clinical trial (Goodale et al., 1990), 46 American women were randomly assigned to one of three groups: a charting group, a reading group, and a relaxation response group. The trial found the relaxation response group in reducing the negative moods of PMS to be superior to the other two groups. In another study (Blake et al., 1998), the results indicated that cognitive therapy was significantly more effective than assignment to a waitlist group, and women in cognitive therapy showed significant improvement in partner relationships, home management, and mood. Morse and colleagues (1991) compared hormone therapy (duphaston), coping skill training and relaxation for the relief of premenstrual syndrome in 42 Australian PMS sufferers, and found that all subjects had benefited from undertaking some form of treatment. Significant improvements were shown in respect of physical and psychological symptoms and state-anxiety initially in the hormone therapy group, whilst coping skills training was found to be superior to both hormone treatment and relaxation training over time and to short-term following up (3 months later). This study showed no positive effects of relaxation alone. Practising relaxation at home alone is considerably more difficult since it requires discipline, organization and motivation of the woman in isolation, and engaging in relaxation may also increase anxiety in some people as they may find the tasks of self attention boring and unpleasant. Hunter and colleagues (2002) conducted a clinical study $(n=45)$ to investigate the difference between medical (fluoxetine) and psychological (cognitive-behavioural therapy) 
treatments for PMDD. They reported that both treatments were equally effective in reducing depressed mood, while fluoxetine treatment had a more rapid effect and greater impact upon anxiety symptoms. Improvements in PMS symptoms were maintained at three months follow-up in Morse and colleagues' trial, for over four months following cessation of therapy in Blake and colleagues' trial, at 9-month follow up in Goodale and colleagues' trial, and one year follow-up in Hunter and colleagues' trial. The cumulative results of these studies have provided various psychological approaches including cognitive-behavioural strategies, relaxation techniques and information on ways to relieve PMS symptoms. These approaches can be a feasible option for women who do not wish to take medications or for use as an adjunct to medical treatment

PMS sufferers may also benefit from aerobic exercise. It has been hypothesized that exercise increases endorphin levels, which in turn improve mood (Johnson, 1998). Prior and colleagues (1987) reported that six months of exercise training had resulted in decreased premenstrual symptoms in two groups of women in their study. The results in Steege and Blumenthal's study (1993) indicated that women with PMS who practiced aerobic exercise reported fewer symptoms than those in the control groups. Although these trials were small in sample size ( $n=21$ and 23), given the associated benefits of exercise e.g. weight control, lower blood lipid levels and blood pressure (Mercy 1991), it seems reasonable to recommend an aerobic exercise for PMS women to help alleviate symptoms.

Nutritional supplements which have proven efficacy that have been evaluated in women with PMS include vitamins, calcium, magnesium, 
multivitamin/mineral supplements, evening primrose oil and chaste-tree berry (Wyatt and colleagues 1999; Kleijnen et al. 1990; London et al. 1987;

Dickerson 2003; Thys-Jacobs et al. 1998; Facchinetti et al. 1991; Walker et al. 1998).

Vitamin $\mathrm{B}_{6}$ has been utilized as a treatment of PMS for many years. There have been many trials worldwide (e.g. Williams et al. 1985; Stewart 1987; London et al. 1991; De Souza et al. 2000) on the use of vitamin $B_{6}$ for this purpose. Wyatt and colleagues (1999) performed a systematic review including a meta-analysis on the topic involving nine randomized, controlled clinical trials of vitamin $\mathrm{B}_{6}$. They concluded that vitamin $\mathrm{B}_{6}$ in doses of 50 to $100 \mathrm{mg}$ per day provides some benefit for patients with PMS. However, some side effects, e.g. peripheral neuropathy, indigestion, and nausea, have also been reported (Harrison et al. 1984; Brush et al. 1988; Kleijnen et al. 1990; London et al. 1991). On the other hand, some researchers (London et al. 1987; Dickerson 2003) have proposed that Vitamin E, an antioxidant, might help to alleviate affective and somatic symptoms in women with PMS when 400 units of it are administered daily during the luteal phase.

Calcium carbonate has been studied as a possible remedy for PMS. A large randomized control trial (Thys-Jacobs et al. 1998) involving 466 moderate to severe PMS sufferers assigned to a calcium carbonate or placebo group, reported a $48 \%$ reduction by the third cycle in the total symptom scores during the luteal phase $(P<.001)$ compared with baseline scores in the treatment group. This finding provides good evidence for the effectiveness of calcium 
carbonate as a treatment for PMS. Ward and Holimon (1999) reviewed research literature from January 1967 to September 1999 to evaluate the efficacy of calcium supplementation in the treatment of premenstrual syndrome. They concluded that calcium supplementation of $1200-1600 \mathrm{mg}$ per day is a sound treatment option in women with PMS. Calcium is also relatively inexpensive and is beneficial in preventing osteoporosis. However, the possibility that renal calculi might develop has been a concern with calcium supplementation (Frye $\&$ Silverman 2000). Magnesium supplementation was also found to be effective in alleviating some of the PMS symptoms in Italy (Facchinetti et al. 1991) and in the United Kingdom (Walker et al. 1998). ACOG practice bulletin (2000) recommends calcium, magnesium and vitamin $\mathrm{E}$ as nutritional supplementation to help resolve PMS symptoms, but advises that vitamin $\mathrm{B}_{6}$ should not be routinely recommended for women with PMS since high dose of this vitamin for prolonged periods of time can cause neurological symptoms.

Dietary recommendations, such as reducing intakes of salt, chocolate, sugar, caffeine tobacco and alcohol, and increasing the consumption of complex carbohydrates are often advised for alleviating the physical and psychological symptoms of PMS (Daugherty 1998; Johnson 1998; ACOG Practice Bulletin 2000; Dickerson 2003). Of these recommendations, only carbohydrate rich beverages during the late luteal phase have been studied in a randomized controlled trial (Sayegh et al. 1995). This study reported that fewer mood changes were experienced by women in the hours following consumption than those who consumed an isocaloric beverage. 
Two herbal supplements, evening primrose oil and chaste-tree berry, have been reported as effective in treating PMS in a review on current literature, with emphasis on more rigorously controlled studies (Hardy 2000). Evening primrose oil contains the essential fatty acid, gamma-linolenic acid, and is widely used as a herbal remedy for breast tenderness. However, a systematic review (Budeiri et al. 1996) of placebo-controlled trials of evening primrose oil found no significant evidence that it is helpful in treating PMS. In recent years, two PMS studies (Schellenberg, 2001; Lauritzen et al. 1997) reported that chaste-tree berry (Vitex agnus-castus) shows some benefit over placebo or vitamin $\mathrm{B}_{6}$ in comparison to baseline. In another study (Atmaca et al 2003), the outcome indicated that patients with PMDD responded well to treatment with combined selective serotonin reuptake inhibitor (SSRI) (fluoxetine) and Vitex agnus castus. This dried ripe fruit of the chaste tree, Vitex agnus castus, has been used for female reproductive complaints since ancient Greece (Brown 1994). In Germany, Vitex agnus castus has a long history of use and is approved by the German Government's Commission E for the treatment of menstrual irregularities and PMS (Blumenthal et al 1998). Side effects seem to be minor including mild gastrointestinal complaints, mild skin rash, increased acne, headaches, and increased menstrual flow, and there are no reported drug interactions of Vitex agnus castus (Tesch 2003). However, fluoxetine was more effective for psychological mood symptoms while the extract was more effective for physical symptoms including breast tenderness, swelling, food craving and cramps (Atmaca et al 2003). 
Chiropractic therapy was reported to be effective in decreasing overall PMS symptoms by Walsh and Polus (1999). However, the scores difference between treatment and placebo groups was only significant in the group that received the active treatment first.

Surgical oophorectomy is an extreme measure as a treatment for PMS. It will give rise, inevitably, to surgical menopause and the woman may experience side effects such as hot flushes, anxiety, fatigue, depression, irritability, headache, heart palpitation, dizziness and osteoporosis. In fact, these symptoms are also PMS symptoms. Surgical oophorectomy is irreversible and involves significant long term risks e.g. heart disease, arthritis and osteoporosis. Also long term supplement of oestrogen and calcium will be required until menopause ensues. It should only be considered after other options have failed and/or there are coexisting gynaecological problems (Casper \& Hearn 1990; Ismail \& O’Brien 2005).

\subsubsection{Pharmacological Interventions of PMS}

Pharmacological agents such as hormones (progesterone, danazol, gonadotropin-releasing hormone $(\mathrm{GnRH})$ agnoists), selective serotonin reuptake inhibitors (fluoxetine, sertraline, paroxetine, fluvoxamine, citalopram, venlafaxine), anxiolytics (benzodiazepine alprazolam, buspirone), diuretics (spironolactone), bromocriptine mesylate, and nonsteroidal anti-inflammatory drugs (NSAIDs) have all been used to treat PMS symptoms (Ugarriza et al 
1998; Frye \& Silverman 2000; Rapkin 2003; Ismail \& O’Brien 2005).

Unfortunately, many of these drugs have several potentially serious adverse effects (Young, 2001) and some have been shown to provide symptomatic relief no better than placebo or for only short periods of time (Freeman \& Rickels 1999).

Selective serotonin reuptake inhibitors (SSRIs) have been the only pharmacological agent clearly shown to be more effective than placebo in treating PMDD. Dimmock and colleagues (2000) recently published a comprehensive systematic review and meta-analysis of 15 randomized controlled trials. Results of the meta-analysis strongly support the effectiveness of SSRIs in the treatment of physical and behavioural symptoms in PMS. It was also found that there was no difference in effectiveness between continuous and intermittent therapy during the luteal phase. Fluoxetine and sertraline are the only two SSRIs approved recently for PMDD by the U.S. Food and Drug Administration (FDA) and by several other countries (Steiner 2000; Rapkin 2003), but at present none of these drugs has a pharmaceutical license for the management of PMS or PMDD in the UK or Europe (Ismail \& O'Brien 2005). However, SSRIs have been reported to produce some side effects such as headache, insomnia, gastrointestinal disturbances, fatigue, nervousness, difficulty in concentration, dizziness, and sexual dysfunction (Steiner 2000; Douglas 2002).

Progesterone has not been found to be effective in the treatment of PMS in spite of long term clinical use in many countries. A systematic review of 14 
randomized controlled trials (Wyatt et al., 2001) found no improvement in overall symptoms among women taking progesterone. Although other hormonal medications, such as danazol and GnRH agonists have been found to be effective for the treatment of PMS (Halbreich et al. 1991; O'Brien \& Abukhalil 1999; Mortola 1991; Sundstrom et al. 1999), side effects limit their long-term use. Continuous danazol therapy may give rise to masculinization, and long-term GnRH treatment can result in osteoporosis and increased risk of cardiovascular disease (Rapkin 2003). The use of oral contraceptives in PMS is limited by the shortage of randomized control trials supporting their efficacy. As well, side effects of oral contraceptive pills including breast tenderness, headache, bloating, and depression are frequently reported by women suffering PMS so it is counter-productive to advocate these as a treatment for relief.

Interestingly, some side effects of SSRIs and oral contraceptives pills are within the same class of complaints for which women will seek help in the first place. Therefore, a key question concerns whether the effects of these pharmacological agents are better or worse than the condition itself. Although anxiolytics such as alprazolam have been shown to be somewhat effective in patients with PMS (Berger \& Presser 1994; Freeman et al. 1995b; Diegoli et al. 1998), treatment using anxiolytic agents is not recommended because of their potential for drug dependence and the variable effectiveness for the relief of PMS symptoms over time.

Spironolactone (Aldactone) is the only diuretic that has been shown to effectively relieve PMS symptoms such as breast tenderness and fluid retention 
(Wang et al., 1995). However, randomised controlled trials (e.g. O'Brien et al. 1979) found no support for spironolactone in well designed studies.

Bromocriptine (Parlodel) has been shown to relieve breast tenderness and menstrual migraine in women with PMS, but side effects also limit its usefulness (Herzog 1997). NSAIDs may alleviate a wide range of physical symptoms and headache, but they do not appear to improve mastalgia (Dickerson et al. 2003). Lastly, all NSAIDs must be used with caution in patients with underlying gastrointestinal or renal disorders.

\subsubsection{Summary}

Currently, SSRIs are the pharmacological treatment of choice for severe PMS/PMDD, and at this time, fluoxetine and sertraline are the only two FDA approved SSRIs for the treatment of PMDD. For women who do not meet criteria for PMDD, or do not wish to take medications, conservative treatments such as psychotherapy, nutritional supplementation and/or diet and lifestyle changes are possible alternative measures. Herbal treatment can be another option. While the strength of evidence is central in treatment decision making for PMS, factors such as adverse effects, potential health benefits, costs, and severity of symptoms should also be taken into account. 
Chapter 3: Traditional Chinese Medical Perspective on Issues for Premenstrual Syndrome

3.1 Definition of Premenstrual Syndrome in Traditional Chinese

Medicine

3.2 History of Premenstrual Disorders in the Chinese

Tradition

3.2.1 Feverish Sensation and Menstruation $\quad 52$

3.2.2 General Body-ache and Menstruation 53

3.2.3 Diarrhoea and Menstruation 54

3.2.4 Oedema and Menstruation $\quad 54$

3.2.5 Headache and Menstruation $\quad 55$

3.2.6 Abdominal Cramps and Menstruation 55

3.2.7 Emotional Changes and Menstruation $\quad 55$

3.2.8 Dizziness and Menstruation $\quad 56$

3.2.9 Other Symptoms $\quad 56$

$\begin{array}{lll}3.2 .10 & \text { Summary } & 57\end{array}$

3.3 Theories of Aetiology and Pathogenesis of Premenstrual 57 Syndrome in Traditional Chinese Medicine

3.3.1 Physiology of Menstruation and PMS $\quad 57$

3.3.2 Mal-Functioning of the Liver System and PMS 58

$\begin{array}{lll}\text { 3.3.3 Individual Constitutions and PMS } & 59\end{array}$

$\begin{array}{lll}\text { 3.3.4 Organ Systems and PMS } & 60\end{array}$

3.3.5 Summary 64

3.4 Diagnosis of Premenstrual Syndrome in Traditional Chinese 65 Medicine

3.4.1 TCM Diagnostic Methods $\quad 65$

3.4.2 Diagnosis of PMS in China 71

3.4.3 Diagnosis Standards in TCM Studies in China 72 
3.5 Classification of TCM Patterns inPremenstrual Syndrome

3.5.1 TCM Patterns according to General Manifestation of PMS 78

3.5.2 TCM Patterns according to Specific Symptoms of 82

Peri-menstrual Disorders

3.5.3 TCM Patterns according to the Primary Causes 86

$\begin{array}{lll}3.5 .4 & \text { Summary } & 88\end{array}$

3.6 TCM Treatment Approaches inPremenstrual Syndrome 88

3.6.1 Treatment for the Syndrome of PMS 89

3.6.2 Treatment for Specific Symptoms of PMS 92

3.6.3 Treatment for the Organ Systems 100

3.6.4 Treatment according to Menstrual Cycle Phases 103

3.6.5 Treatment with Integrated Traditional Chinese and Western 104

Medicines

3.6.6 Types of TCM Herbal Formulas and Herbs 106

3.6.7 Outcome of Herbal Treatment Trials in PMS 120

$\begin{array}{lll}3.6 .8 & \text { Other Therapies } & 120\end{array}$

$\begin{array}{llr}3.7 & \text { Summary } & 124\end{array}$ 


\subsection{Definition of Premenstrual Syndrome in Traditional Chinese Medicine}

Western medicine defines Premenstrual Syndrome (PMS) as a collection of cyclical physical and mental symptoms in women, but traditional Chinese medicine (TCM) thinking does not consider it as a single distinct disorder. According to the view of traditional Chinese medicine, a focus on individual symptoms around the menstrual period predominates. Descriptions of specific symptoms which are associated with menstrual periods such as "oedema during menstruation", "premenstrual breast distension", "headache during menstruation" and "premenstrual emotional changes" can be found in various Chinese medical publications (SiTu \& Yang 2000). TCM defines and discusses menstrual related disorders that are symptom-based rather than proceeding along a syndrome perspective as modern western medicine. The description of these disorders is similar to those "older medical writings" of western society that did not distinguish between premenstrual and menstrual complaints (Richardson 1995)

Today, some gynecologists in Chinese medicine classify all these symptom disorders collectively as 'Peri-menstrual Disorders', a term that first appeared in 1980 (Hubei college of TCM 1980). Typical descriptions of peri-menstrual disorders, Xia (1994) refer to a series of menstrually related disorders that are cyclical and recurrent, and in which a symptom/range of symptoms appears a few days before and/or during menstruation, may continue until the cessation of 
menstruation, and disappears or reduces to a negligible level after commencement of menstruation. Since the definition of 'Peri-menstrual Disorders' in Chinese medicine relates to the cyclical timing of symptoms before, during and after menstruation, some other conditions that are associated with disorders other than PMS, e.g. period pain, endometriosis, chronic pelvic infection, and general blood deficiency may also be included. While TCM advocates no significant difference in the treatment process of these disorders, confusion may arise among western and modern Chinese scientific views and reports regarding the exact nature of the condition being considered.

Some gynecologists in Chinese medicine continue to categorize these disorders according to individual symptoms, following many classic texts, such as The Gynecology of TCM (Luo 1986) and The Gynecology of TCM (Ma 1997). Both proposed 11 different disorders that are symptom-based, i.e. 'Breast distension during menstruation', 'Feverish sensation during menstruation', 'Headache during menstruation', 'General bone-ache during menstruation', 'Diarrhoea during menstruation', 'Haematemesis during menstruation', 'Oral ulcer during menstruation', 'Rashes during menstruation', 'Dizziness during menstruation', 'Oedema during menstruation' and 'Emotional changes during menstruation' . The above definitions of the disorder relate in particular to the cyclical timing of symptoms, without characterizing its special timing (premenstrual phase) of menstruation and severity level. Thus, the Chinese medicine definition is less specific, more inclusive and may potentially confuse understanding. 
Therefore most research of premenstrual disorders in Chinese medicine in recent years and in some recently published gynaecology books in fact adopt the definition of PMS from western medicine and only those symptoms which appear before and during menstruation are considered. Sun and Guo (2004), Zhou Z (2003) and Wang B (2002), follow the western view of definition and refer to PMS as a group of cyclic changes that include somatic, psychological and behavioral disturbances which appear recurrently in the late luteal phase (days 24-28) of the menstrual cycle. These disturbances must be severe enough to cause pain or distress in the woman's mind and/or body and adversely affect the woman's daily life and work. These symptoms must also resolve naturally after menstruation. In a TCM gynecological textbook, the Women Diseases TCM Clinical Diagnosis and Treatment (SiTu and Yang 2000), premenstrual tension syndrome refers to the physical, psychological and behavioural changes during the 7-10 days before menstruation. These symptoms may affect the woman's normal life and work, and disappear naturally after menstruation. These defining characteristics are based on western medicines.

Both premenstrual syndrome and premenstrual tension syndrome have been used synonymously to describe this disorder in many Chinese medical articles and text books regardless whether or not nervous tension is a common symptom of the disorder. 


\subsection{History of Premenstrual Disorders in the Chinese Tradition}

The earliest records and discussions of symptoms during premenstrual and menstrual phases in Chinese classics date back to the Ming Dynasty (1368-1644AD). In The Appendix of Dan-Xi's Experiential Methods (Fang 1536), the author indicated that to diagnose cases of 'feverish sensations' in women's diseases, one should ascertain whether the feverish sensations occurred during menstruation or also at other times. In the Qing Dynasty (1644-1911AD), as many as 22 premenstrual symptoms were listed in the $Y e$ Tian-Shi's Gynaecological Records (Liu 1921). Most of the symptoms discussed in Master Ye's book were somatic problems such as oedema, feverish sensations, hypochondriacal pain, diarrhoea, body aches, abdominal cramps, and reduced appetite, except one which was manic or 'delirious speaking'.

The description and discussion on disorders during menstruation in TCM classics are grouped under different symptoms.

\subsubsection{Feverish Sensation and Menstruation}

The Elementary Course for Medicine (Li 1624), proposed that the feverish sensations and cramps during menstruation were common disorders among women, and when feverish sensations occur before menstruation these are due 
to Blood deficiency with stagnation, and feverish sensations after menstruation is due to Blood deficiency with Heat.

In the Golden Mirror of Medicine (Wu 1742), fever/feverish sensations during menstruation were considered to be due to Heat in the Blood, whilst fever/feverish sensations after menstruation were due to a deficiency. If the feverish sensations happened continually, it was believed to be caused by pathogens, and if they only happened in the afternoon, it was believed to be the result of Yin deficiency.

\subsubsection{General Body-ache and Menstruation}

The Standards of Diagnosis and Treatment: Gynecology (Wang 1602) recorded that general body-ache was one of the symptoms which indicated that menstruation was imminent.

The Complete Book of Ye's Obstetrics and Gynecology (Ye 1913), pointed out clearly that the general bone-ache in the first 2-3 days of menstruation was likely caused by a Cold pathogen entering the bone.

The Golden Mirror of Medicine (Wu 1742) proposed that diagnosis of bone-ache during menstruation should be ascertained if it was caused by deficiency/excess and Wei/Ying. Wu believed that bone-ache happened with sweating but no swelling was regarded as due to insufficiency of Wei Qi 
(Protective Qi); if there was swelling but no sweating then it was believed to be due to the excess of Ying Qi (Nourishing Qi).

\subsubsection{Diarrhoea and Menstruation}

In the Fu Qing Zhu's Obstetrics and Gynecology (Fu 1826), Master Fu suggested that women suffering from diarrhoea three days before menstruation was a indication of Spleen deficiency and not as some other practitioners believed to be caused by excess of Blood.

In the Complete Book of Ye's Obstetrics and Gynecology (Ye 1913), Master Ye described early morning diarrhoea during menstruation as a sign of Kidney deficiency.

Both The Supplementary and Explanatory Notes of Chen Su-an's Gynecology (Chen Ming Dynasty, 1368-1644) and Golden Mirror of Medicine (Wu 1742) claimed that diarrhoea during menstruation was caused by Spleen deficiency.

\subsubsection{Oedema and Menstruation}

In the Complete Book of Ye's Obstetrics and Gynecology (Ye 1913), Master Ye explained that general oedema/swelling of the body during menstruation is 
caused by a weakened Spleen that fails to transport and transform water which is then retained in the body resulting in swelling.

\subsubsection{Headache and Menstruation}

Zhang's Treatise on General Medicine (Zhang Lu 1695) considers that headache, distension, palpitation, reduced appetite and dull skin during every menstruation resulted from the Phlegm-Dampness which are pathological products of impaired water metabolism.

\subsubsection{Abdominal Cramps and Menstruation}

Golden Mirror of Medicine (Wu 1742) stated that abdominal pain before menstruation was due to $Q i$ and Blood stasis, whilst weakness of $Q i$ and Blood may lead to abdominal pain after menstruation.

\subsubsection{Emotional Changes and Menstruation}

The Supplementary and Explanatory Notes of Chen Su-an's Gynecology (Chen Ming Dynasty 1368-1644) explained that emotional changes during menstruation were the result of the deficiency of Blood in the body giving rise to internal Heat and manifesting as emotional changes. The woman required to 
be treated with Blood-cooling herbs to clear the Heat while diaphoretic herbs should be avoided because water would be lost from the body.

\subsubsection{Dizziness and Menstruation}

In The Supplementary and Explanatory Notes of Chen Su-an's Gynecology (Chen Ming Dynasty 1368-1644), the pathogenesis of dizziness during menstruation is discussed. It is considered that the heavy headed sensation was due to the sinking of Yang $Q i$, and blurring of vision was caused by the lack of Jing and Blood.

\subsubsection{Other Symptoms}

In addition to the above, the Classified Treatise on Obstetrics and Gynecology (Xiao 1689) recorded additional symptoms which included body-ache, feverish sensation, blurring of vision, and diarrhoea during menstruation. The Excerpt of Chen's Obstetrics and Gynecology (Chen 1850) also mentions hoarseness of voice and blurring of vision during menstruation. 


\subsubsection{Summary}

While the Hippocrates described these menstrual symptoms as uterine gaseous distension and associated the etiology of the condition to the accumulation of black bile in the uterus (Ricci 1950, cited in Richardson 1995), most of the descriptions and discussions of all disorders during menstruation in TCM classics claim that these peri-menstrual disorders are caused by the malfunctioning of Zang-fu, Qi or Blood, such as Liver stagnation, Spleen deficiency, Blood deficiency, and Kidney deficiency, but some conditions may also be related to excess Heat, Cold pathogen and/or Wind-Coldness. Also, the latest recommended treatment method of PMS suggests that medications be

given to treat specific symptoms and that treatment be individualized to target the most troublesome symptoms in each patient (Dickerson 2003). Interestingly, this strategy has a similar initiative to the historic TCM treatment.

\subsection{Theories of Aetiology and Pathogenesis of Premenstrual Syndrome in Traditional Chinese Medicine}

\subsubsection{Physiology of Menstruation and PMS}

Menstruation is a natural physiological phenomenon which, as proposed by TCM, is produced as a result of the action of blood, $Q i$ (energy force), Tiankui (Yin-essence), meridians (especially the Chong and Ren meridians), and internal organs affecting the uterus (Chen 1998). The occurrence of the 
symptoms of PMS before menstruation, diminishing gradually as menses begins or after menstruation has ceased, is proposed to be caused by the changes of the deficiency and excess of Blood and $Q i$, the emptiness and fullness of the Chong and Ren meridians, and the body constitution of the individual (Cai 1977; Chen 1998). Prior to menstruation, blood and $Q i$ travel down to the uterus via the Chong and Ren meridians for the preparation of menstruation. When $Q i$ is abundant, blood will flow more rapidly, often resulting in an accumulation of blood causing blockage of the meridians. As the Chong and Ren meridians are governed by the Liver meridian, Liver Qi will be disrupted (Han 1998). The accumulation of blood in the lower part of the body (uterus area) will result in an imbalance of yin-blood in the remaining areas of the body. Consequently, the function of those internal organs will also be adversely affected leading to the systemic signs and symptoms associated with PMS (Luo 1986). The associated symptoms disappear once the balance is restored upon the commencement of menstruation.

\subsubsection{Mal-Functioning of the Liver System and PMS}

Women are regarded as being prone to a yin-blood deficiency because of regular blood losses during menstruation, pregnancy and delivery. When the internal organs (especially the Liver, the Spleen and the Kidney) are lacking in nourishment of blood, their function will be impaired. The Liver system is the reservoir of blood and regulator of blood and $Q i$ for the entire body. Failure to properly regulate and distribute blood in the appropriate quantities throughout 
the body is hypothesised to result in the development of disharmonies. Besides being the regulator of the Blood system, the Liver system also ensures the smooth flow of $Q i$ throughout the body. If the function of the Liver system is impaired, the harmonious movement of $Q i$ will be obstructed. When $Q i$, which is the basis for all physical, emotional and mental processes, becomes restrained, it will give rise to emotional and physical symptoms (Maciocia 1989). The Liver meridian passes through the head, face, breasts, hypochondrium and lower abdomen. It can therefore be deduced that the common symptoms of PMS such as headache, dizziness, breast tenderness or distension, irritation, agitation, frustration, depression and anger are caused by the mal-functioning of the Liver system (He \& Wang 1995) which, in TCM, is the basic contributing factor for PMS (Wu 1985; Yu 1997).

\subsubsection{Individual Constitutions and PMS}

Individual constitutions of women also have an important place with the idiosyncratic nature of PMS symptoms (Xiao 1999). The degree of deficiency in $Q i$, Blood, Yang and Yin, and the excess condition of individual organs/constitutions i.e. Fire, Stasis and Dampness, may give rise to different symptoms. The five elements theory of TCM in the Yellow Emperor's Classic of Internal Medicine (circa, 100BC), proposed that the physiology and pathology of all the internal organs of a human body are interconnected and interdependent, and one organ may affect other organs depending on the organ's state of health and functional capability. 
The proposed etiology of PMS in TCM shares a similarity to the somatic-weakness theory of psychosomatic disorders which proposes that each person's body has a genetically determined weak link in system which breaks down when the person is under stress (Herman \& Lester 1994). Likewise, TCM proposes that the imbalances in the Blood, Meridian and Liver systems before and during menstruation induce the break down of the weak link (area of deficient) in the body constitution of the individual giving rise to the various symptoms of PMS.

\subsubsection{Organ Systems and PMS}

The variation of symptom manifestation and symptom severity in PMS can be explained as being related to the particular constitution of the woman and the state of health of her various organs. However, TCM practitioners, according to their clinical experiences, have different views in explaining the aetiology and pathogenesis of different symptoms of PMS.

\section{$\underline{\text { Liver system and PMS }}$}

Most Chinese medicine researchers agree that Liver $Q i$ stagnation is the primary cause of PMS. Emotional disturbances may cause the stagnation of Liver $Q i$ (Maciocia 1989), and pathophysiological changes during the premenstrual period may exacerbate the disorder. When Liver $Q i$ is restrained 
within the meridian, it may give rise to symptoms such as irritation, agitation, depression, anger, breast tenderness and/or distension.

Cai (1997), Wang (2000), and Zhang (2005) propose that symptoms of PMS are mainly caused by the stagnation of $Q i$ and the disharmony between the Chong and Ren meridians during menstruation. Huang (1999) and Tang (2005) believe that Liver $Q i$ stagnation is the main cause of PMS. Long term stagnation of Liver $Q i$ either leads to $Q i$ and Blood stagnation, or affects other organs and gives rise to different symptoms accordingly.

Since the Liver meridian passes through the breast and nipple, Tian and colleagues (2003) and $\mathrm{Bi}(2004)$ consider that premenstrual breast pain is due to constrained Liver $Q i$ in the meridian.

Prolonged stagnation of Liver Qi may transform into Heat/Fire (Deng 1999). The upflaming of Liver-Fire/Yang rises to the head along the Liver meridian, causing symptoms such as headache, dizziness, anger, and frustration. Zhong and Chen (2001) believe that $Q i$ and Blood stagnation of the Liver and the route of the Liver meridian is the reason for premenstrual headaches. Ding and colleagues (2001) believe that Liver stagnation and Liver Fire are the causes of premenstrual tension. Chen and Zhao (1998) consider that the agitated type of PMS is due to Liver $Q i$ stagnation that transforms into Fire. Liver Fire causes Yin deficiency and Liver exuberance and gives rise to the outward symptoms, and the depressed type of PMS is due to restrained Liver $Q i$ attacking the 
Spleen, causing deficiency and weakness of the Spleen and Stomach that in turn gives rise to the inward symptoms.

\section{Spleen and Stomach systems and PMS}

Overwork and too much worrying may damage the Spleen system which governs digestion and generates $Q i$ (Maciocia 1989). In the case of PMS, Li (1999) acknowledges that stagnant Liver $Q i$ may disrupt the Spleen. A weak (an insufficient) Spleen system becomes more deficient and cannot transform and transport Water/Dampness inside the body. As Water/Dampness is retained in the skin and muscles, it will manifest as oedema (Liu 2000). When Water/Dampness moves downward and is retained in the Large Intestines, it may give rise to diarrhoea ( $\mathrm{Li} 2000$ ). If $Q i$ is deficient and dampness is retained in the body, it may give rise to tiredness, fatigue, general weakness, hypersomnia, dizziness and/or proneness to infection (Xiao 1999).

When the Fire/heat in the Liver is transferred to the Stomach system, the heat affects the Stomach's governing of intake, thus causing swift digestion and manifesting as increased appetite and rapid hungering (Song 1996). If the down-bearing of Stomach $Q i$ is affected, it may give rise to nausea and vomiting (Wu \& Zhang 2001).

\section{$\underline{\text { Kidney system and PMS }}$}

Kidney and Liver are known to have a very close relationship. Liver stores blood while Kidney stores Jing-essence (Maciocia 1989). When blood fails to nourish the Kidney system, the Kidney $Q i$ which includes both Kidney Yang 
and Yin is impaired. Kidney Yang is the root of Yang Qi and provides warmth for the organs of the entire body (Deng 1999). Both Liu (2000), Zhang and Li (2004) also point out that an insufficient Kidney Yang which fails to warm the Spleen and transform water may further advance oedema and diarrhoea in PMS.

In the case of Kidney Yin insufficiency, both Yin-blood and essence are further deficient and may fail to nourish the internal organs during premenstrual period, and all associated symptoms may become more severe. Zhou (1999) and Zhu (2001) acknowledge that Liver and Kidney insufficiency may lead to $Q i$ and Blood stagnation in the meridians as well as failure to nourish the brain causing headaches. Kidney-Yin deficiency may generate internal Heat/Fire. This deficient Heat may give rise to hot flushes and may advance those symptoms which are caused by Heat, such as headaches, irritability, oral ulcer, body rashes, and insomnia (Peng 1999).

\section{Heart system and PMS}

Liver Fire/Heat may affect the Heart, transferring Heat/Fire in the Heart system thus giving rise to symptoms such as oral ulcer, irritability, frustration and insomnia (Gao 2005). If the Blood fails to nourish the Heart system, manifestations such as forgetfulness, palpitation, crying, insomnia or lethargy may occur since the spirit of the body is controlled by the Heart system (Cai \& Zhou 1997; Gao 2005). 


\section{Qi and Blood systems and PMS}

Regular blood losses during menstruation may render women more susceptible to Yin-blood deficiency. Further deficiency in $Q i$ and Blood before and during menstruation increases the chances of pathogenic invasion.

Zhou and colleagues (2002) propose that the appearance of rashes and itchiness during menstruation is caused by the deficiency of Blood and the external attack of pathogenic Wind that gives rise to such symptoms. Lin (2000) considers that headaches during premenstrual and menstrual period are due to the invasion of Coldness. Pathogenic Cold slows the movement of $Q i$ and congeals the Blood in the meridians in the head region giving rise to headaches.

\subsubsection{Summary}

The above theoretical views of TCM seek to explain the appearance and disappearance of PMS symptoms in different menstrual phases, and also the idiosyncratic nature of PMS complaints. However, since these TCM theories were conceptualized as the interaction between human body and the universe, it is difficult for these theories to be tested using western research methods. Although some experimental studies have been carried out, for example the study on the ratio between cAMP/cGMP and the balance of Yin and Yang (Kuang et al. 1984), and the studies (Hu \& Mo 1998; Zhou \& Zhu 1984; Huang $\&$ Zhou 1998) on the relations between the deficiency of Heart $Q i$ and Serum Endogenous Digitalas like Factor (sEDF), Serum $\mathrm{TXB}_{2}$ 6-keto-PGFa and 
micro-elements (zinc, copper, iron and calcium), these studies were fairly primitive and limited in scope.

\subsection{Diagnosis of Premenstrual Syndrome in Traditional Chinese Medicine}

\subsubsection{TCM Diagnostic Methods}

TCM diagnosis is based on the concept that the human body is an organic integral whole. The body consists of a number of internal organs, tissues and meridians with different functions. The functions of all internal organs and tissues are interconnected with meridians distributed over the entire body, which together are presumed to maintain the harmony of the physiological activities of the body. Meridians run on the surface of the body, enter their

pertaining organs and are linked with one another, and as such they connect the exterior, interior, upper and lower portions of the body (Deadman et al. 1998). Any disharmony within the internal organs is reflected by external signs and symptoms, and detailed information about the state of the whole body can be derived from examination of local areas. These are known as "Inspecting the exterior to examine the interior" and "A part reflects the whole" (Maciocia 1989). TCM diagnosis therefore considers not only the chief signs and symptoms, but all the other manifestations of the disorder (Zhang 1990). 


\section{Pattern Identification}

The TCMdiagnostic process is known as 'Pattern Identification' which is called Bian Zheng in Chinese It involves a synthesis of all symptoms and signs that are collected through the four methods of diagnosis in a meaningful pattern of disharmony to guide subsequent treatment (Maciocia 1989). Pattern Identification of disharmony blends diagnosis, pathology and treatment principles all into one. It allows the practitioner to identify the nature, the character, the location, the treatment principle and the prognosis of the condition. There are several methods that can be used to identify patterns. These include:

- Pattern Identification according to the Eight Principles (Yin,

Yang ,Exterior, Interior, Coldness, Heat, Excess and Deficiency),

- Pattern Identification according to the state of Qi and Blood,

- Pattern Identification according to the state of the internal organs,

- Pattern Identification according to pathogenic factors,

- Pattern Identification according to the Twelve Meridians,

- Pattern Identification according to the Six Channels, and

- $\quad$ Pattern Identification according to three Burners and four Stages (Wei, Qi, Ying and Xue)

These methods can be applied on their own individually or together in different situations. The last two methods are seldom used for the diagnosis of PMS.

\section{Disorder Identification}

Pattern Identification groups the signs and symptoms into one or more patterns of disharmony. One disorder may include several different patterns of 
disharmony, while the same pattern may appear in different disorders. A complete TCM diagnostic process also involves the diagnosis of the disorder. Identification of a disorder suggests the entire course of pathological changes, where the pattern generalizes the pathology of a disorder in its certain stage. Combining identification of disorder with identification of patterns, the nature, progress and prognosis of the disorder can be positively ascertained thus enabling correct treatment and prevention to be carried out. The name of a TCM disorder is usually defined according to either it's causes, locations or symptoms.

\section{The Four Methods of TCM Diagnosis}

The four methods are inspection, auscultation and olfaction (these two words are the same word in Chinese), interrogation and palpation. By combining the information obtained through the four diagnostic methods, a comprehensive and systematic understanding of the situation and nature of the disorder can be gained and a correct diagnosis can be made.

\section{Inspection}

Inspection is a technique which the practitioner uses to collect information about the exterior condition of the patient's health and illness through observation (looking). This includes observation of the spirit, complexion, general appearance, demeanour, tongue, skin, secretions and excreta. Colour is one of the important diagnostic pointers for inspection. In TCM, the five colours are corresponding to the five elements. For example, colour red pertaining to fire, indicates a Heat syndrome which includes either excess or 
deficient Heat in the body. In PMS, many symptoms such as headache, anger, irritability, frustration, increased appetite, oral ulcer, and insomnia are associated with Heat. Observation of a red complexion/flush face, malar flush, red tongue, red tongue tip/sides, red eyes or red lips of the women may assist the TCM practitioner to identify the patterns of PMS.

\section{Auscultation and Olfaction}

Auscultation involves listening to the sound of the patient's voice, breathing, coughing, vomiting, hiccup, borborygmi, groaning and the sound that expresses pain and discomfort. Variation of sounds reflects the condition of the patient's Qi, Blood, Lung system, Heart system, Kidney system and the harmony between organs. A loud sound usually indicates an Excess pattern and a weak sound indicates a Deficiency pattern.

Olfaction is the diagnostic method to smell the abnormal odour of the patient. It involves smelling of the patient's breath, body smells, secretion and excreta. Foul smell is indicative of Heat or Damp-Heat pattern. Fishy smell is usually found in Cold or Damp-Cold pattern, and some extraordinary smells indicate certain diseases.

\section{Interrogation}

Interrogation is the discussion between the practitioner and the patient, which is the most important technique of TCM diagnosis. Since the onset, progress, course of treatment received, and present symptoms are important for the diagnosis of a disorder, all of this information can be derived through 
questioning. The interrogation involves asking the patient's chief complaint, history of the present disease and the traditional ten areas of questioning (1. Chills and fever, 2. Sweating, 3. Head and body, 4. Stool and urine, 5. Diet and taste, 6. Chest and abdomen, 7. Hearing and vision, 8. Sleep, 9. Past illness, 10. Possible cause: such as life style, emotion, living and working environment). In the case of PMS, clients are female patients, therefore questions concerning menstruation, pregnancy and childbirth should be included.

\section{Palpation}

TCM palpation includes pulse examination and general palpation.

\section{Pulse examination}

Pulse examination is the technique by which the practitioner judges the patient's condition by feeling the patient's arterial pulse with fingers. In TCM, the blood vessels are the house of $Q i$ and blood. It is possible to assess the patient's condition and any changes by feeling the length, size, rate and rhythm of the pulse to determine the state of $Q i$ and Blood of the body. Palpating the pulse on different positions and depths also provide information on the location and nature of the disorder. Other than the normal pulse, there are 28 common pulse images which reflect different conditions in the body. Wiry pulse, the most common pulse in PMS, indicates that there is either disharmony of the Liver and the Gallbladder, all kinds of pain, Phlegm retention, or malaria (Wiseman 1994). Since TCM believes that the Liver is the organ which governs and regulates the free flow of $Q i$ in the body, that pain indicates blockage, and that Phlegm retention may cause obstruction in the body, a wiry 
pulse can be interpreted as the manifestation of the retention of $Q i$ in the meridians. Liver $Q i$ stagnation is the primary cause of PMS, hence wiry pulse is often detected in PMS women.

Thin pulse indicates that there is deficiency of $Q i$, Blood or Yin-fliud which fails to replenish or fill the blood vessels, or Dampness retention in the body that obstructs the vessels; slippery pulse indicates retention of Phlegm-fluid, food and Damp-Heat in the body; choppy pulse has an uneven and thin flow which indicates the deficiency of $Q i$, Blood, Yin and/or Jing, stagnation of $Q i$, Blood stasis, accumulation of Phlegm or retention of food; rapid pulse indicates Heat in the body, if the pulse is rapid but without strength, it can be a deficiency pattern; and weak pulse indicates deficiency of $Q i$ and Blood (Wiseman 1994). Since the deficiency of Qi, Blood and Yin, the retention of Damp, Phlegm, and Heat, and Blood stasis are common causes of PMS, these pulses are also very commonly seen in PMS.

\section{General palpation}

General palpation involves feeling of the surface with hands to determine the heat, cold, moistness, texture, distension, pain and reaction to pressure. The examination consists of five parts: palpitation of the skin, the limbs, the chest, the abdomen and the acu-points. 


\subsubsection{Diagnosis of PMS in China}

The diagnosis of 'Peri-menstrual Disorders' in Chinese medicine is made according to the cyclical timing of symptoms that are associated with menstruation, using information collected through the four diagnostic methods during consultation. Traditionally, TCM practitioners diagnose peri-menstrual disorders into patterns of disharmony within each major symptom disorder. From the last century, some practitioners follow the western classification of diseases and recognise peri-menstrual disorder as a TCM disorder. They diagnose peri-menstrual disorder into patterns according to general complaints.

Since peri-menstrual disorders include disharmonies which appear before, during and after menstruation, they may cover some conditions that are associated with disorders other than PMS, e.g. endometriosis and dysmenorrhea. While TCM does not differentiate between the diagnosis and treatments of these disorders, confusion may arise among western medical scientists about the TCM approaches regarding their understanding of the Chinese medicine nosology, diagnosis and subsequent treatment. Therefore, most of the researches of peri-menstrual disorders in Chinese medicine in recent years in fact comprise studies of symptoms/disorders of premenstrual syndrome. 


\subsubsection{Diagnosis Standards in TCM Studies in China}

Researchers of peri-menstrual disorders / PMS in Chinese medicine set the diagnosis standard for their studies by following the Guidelines for Clinical Research in New TCM Medicines of the Ministry of Health (1997), the Professional Standards of Chinese Medicine of the State Administration of TCM (1994), the Clinical Diagnosis Standard of Diseases of the Chinese People's Liberation Army (1998), the American Psychiatric Association (APA), National Institute of Mental Health (NIMH), and/or medical text books of western medicine or TCM. However, most researchers did not mention how their diagnosis standards were set in their reports.

\section{The Guidelines for Clinical Research in New TCM Medicines for}

\section{Pre-menstrual tension syndrome, Ministry of Health, People's Republic of}

\section{China (Appendix A)}

The Guidelines for Clinical Research in New TCM Medicines for Pre-menstrual tension syndrome were first established in 1997. A number of new editions of Clinical Research Guidelines have since been published, but they do not include premenstrual syndrome. These guidelines cover the general symptoms of PMS, the western medicine clinical diagnosis standard and TCM pattern identification of the syndrome. They are often adopted by studies which consider this disorder as a syndrome or which follow the western definition of PMS. 
Qian J (1999) set his diagnosis standard according to western medicine clinical diagnosis in the Guidelines for Clinical Research in New TCM Medicines for his study on 32 Pre-menstrual tension syndrome participants with Liver and $Q i$ stagnation.

All 21 cases in Chen's study (2005) met the symptoms and TCM pattern identification of the Guidelines. There were 13 cases of Liver stagnation transforming into Heat, 2 cases of $Q i$ and Blood stagnation, 4 cases of Yin deficiency with Hyperactivity of Yang, one case of Yin deficiency with Hyperactivity of Fire and Spleen and Kidney Yang deficiency.

Zhu and Xu (2003) set the diagnostic standard for their study according to the Guidelines for Clinical Research in New TCM Medicines for Pre-menstrual tension syndrome and the Gynaecology and Obstetrics (Le, 1997). The diagnosis standard includes: 1/ at least four of the following symptoms appear within 14 days before menstruation: sore and distended breasts, headache, migraine, anxiety and agitation, swelling of face and eye-lid, general aches, difficulty in concentrating, dry month and thirst. $2 /$ the symptoms are related to the women's menstrual cycle and disappear after menstruation. The symptoms have to be present during at least three consecutive cycles and they must be severe enough to disrupt the woman's work or study. 3/ at least two abnormal findings in: estradiol / progestin ratio, premenstrual prolactin $>30 \mathrm{ng} / \mathrm{ml}$, examination of vegetative nerve function, or BBT (Basal Body Temperature) showing a luteal phase defect. However, the researchers did not mention how to measure these variables in detail. 
The diagnostic criteria of the Guidelines for Clinical Research in New TCM Medicines for Pre-menstrual tension syndrome take into account symptoms which appear 7-14 days before menstruation, as compared to a much shorter period of only 5-7 days before menstruation in the diagnostic standards in western society, i.e. ACOG, NIMH and APA. Consequently, the scope of the study is unnecessarily widened as those women who have symptoms in the early luteal phase are also included.

\section{Professional Standards of Chinese Medicine, State Administration of}

\section{Traditional Chinese Medicine, People's Republic China (Appendix B)}

There are only seven symptoms associated with the peri-menstrual period mentioned in this professional standard of Chinese medicine . These are peri-menstrual breast distension; feverish sensation; headache; bodyache; diarrhoea; oral ulcer and bleeding that are recorded in the Criteria of Diagnosis and Therapeutic Effect of Gynaenecologic Diseases and Syndromes in Traditional Chinese Medicine in the Professional Standards of Chinese Medicine, People's Republic of China. However, peri-menstrual bleeding is usually caused by endometriosis and is not associated with PMS. Diagnostic criteria and pattern identification of these peri-menstrual symptoms are clearly stipulated in the standards which are often followed by studies that adopt the traditional classification method according to specific symptoms of peri-menstrual disorders. 
By applying these diagnostic criteria, Zhang and Li (2004) diagnosed 52 clients in their study of peri-menstrual diarrhoea. The modified Shen Ling Bai Zhu San was used to treat both the patterns of Spleen and Kidney Yang deficiency.

Again, in the study of Qiao and colleagues (2002), their PMS diagnosis standard followed the Guidelines for Clinical Research in New TCM Medicines for Pre-menstrual Tension Syndrome. However, the pattern identification standard for Liver $Q i$ Invasion in the PMS study was set according to the Professional Standards of Chinese Medicine, State Administration of Traditional Chinese Medicine, People's Republic China.

\section{Criteria of Clinical Diagnosis and Standard of Therapeutic Effect on Diseases, the Health Ministry, General Logistics Department, the Chinese \\ People's Liberation Army}

The criteria of diagnosis under the topic of Premenstrual Tension simply state

that symptoms such as headache, dry mouth, insomnia, agitation, distended and sore breasts, swelling over face and limbs should appear one week before menstruation, and should disappear automatically after commencement of menses. No criteria on the severity of the disorder or TCM patterns are mentioned

Tan and Li (1999) diagnosed the participants suffering from premenstrual oral ulcer in their study based on the Criteria of Clinical Diagnosis of Premenstrual Tension, Health Ministry, Chinese People's Liberation Army. All 180 cases of 
Premenstrual Tension syndrome in Yu and Liu's study (2004) met the standard set in the Criteria of Clinical Diagnosis of Premenstrual Tension.

\section{Western Medicine Associations}

Li and Colleagues (2002) examined 600 cases of PMS and Yue and Chen (2005) examined 500 cases of PMS. Both studies set their diagnostic standards according to the criteria in the Diagnostic and Statistical Manual of Mental Disorders (DSM-IV), $4^{\text {th }}$ edition of the American Psychiatric Association (APA) and the National Institute of Mental Health (NIMH). NIMH suggests that the diagnosis of PMS should be dependent on the occurrence of an increased symptom severity by at least $30 \%$ in the six days before menstruation compared with that up to five days after, and these symptoms have to be present during at least two consecutive cycles (Dickerson et al. 2003). The DSM-IV (APA 1994) requires the presence of at least five specified symptoms for premenstrual dysphoric disorder (PMDD), which is a severe form of PMS. However, there was no mention of any inclusion or exclusion criteria in detail, or the form of PMS in both study reports.

Of all the Chinese studies discussed in this research, the above two studies had the largest sample size of subjects. It would appear that Chinese researchers of studies with a large sample size pay more attention to or are more likely to be influenced by western medical standards. 


\section{TCM Text Books}

In 2002, Zhou set the diagnosis standard for premenstrual painful and distended breasts according to The Gynecology of TCM (Lo 1986), while Yang set the pattern identification standard for peri-menstrual abdominal pain according to the Chinese Internal Medicine (1985).

Jiang and colleagues applied the diagnostic criteria and the pattern identification on premenstrual tension syndrome according to the text book Practical Diagnosis and Treatment-Integration of Western and Chinese Medicine (Chen \& Yang 1991). They assigned the participants into three TCM patterns:

(1) Liver stagnation: irritability, agitation, hypochondriac/breasts pain and distension, headache, dizziness, red tongue and slippery pulse.

(2) Spleen deficiency: tiredness, fatigue, premenstrual diarrhoea, premenstrual swelling, pale and plump tongue, and slippery pulse.

(3) Blood deficiency: Heavy headed, headache, irritability, insomnia, feverish sensation during menstruation, pale tongue, deep, thin and weak pulses.

\section{Western Medicine Text Books}

Both Li and colleagues (2003), and Wang and Yin (2004) selected their subjects according to the Gynaecology and Obstetric (Le, 2000). Hong (2002), and Sun and Guo (2004) made the diagnosis of PMS according to the text book Modern Gynecology in Chinese and Western Medicine (Niu 1996). The diagnosis standard of premenstrual tension syndrome in An and colleagues' 
study (2005) was set according to Diagnosis and Differential Diagnosis of Gynecological and Obstetrical Diseases (Ding 1999).

These studies either investigated the efficacy of patent herbs or the effectiveness of acupuncture treatment on PMS. It seems that acupuncturists and researchers who would like to promote their products are more reliant on western medical information.

\subsection{Classification of TCM Patterns in Premenstrual Syndrome}

Chinese medicine researchers do not always adopt the same approach for pattern classification in their studies. Some identify patterns from the whole syndrome, others do so based on individual symptoms, whilst a number of researchers consider the best strategy is to identify and apply treatment to the hypothesised root causes of the disorder.

\subsubsection{TCM Patterns according to General Manifestation of PMS}

In a clinical report (Xu 1982) of 170 cases of PMS, treatment was applied according to Pattern Identification. The cases were grouped into 4 patterns, i.e. Liver $Q i$ stagnation - 117 cases; Heat in the internal organs - 35 cases; $Q i$ and Yang deficiency - 16 cases; Blood and Phlegm stagnation - 2 cases. Another PMS study (Cheng et al) in the same year, grouped 102 clients into six TCM 
patterns, i.e. Liver stagnation with Kidney deficiency (30 cases); Disharmony between Liver and Spleen (24 cases); Liver $Q i$ stagnation (7 cases); Liver stagnation transforming into Heat (17 cases); Yin deficiency with hyperactive Liver (16 cases); Spleen and Kidney Yang deficiency (8 cases). The patterns identified in these two early studies became the groundwork for today's pattern identification in PMS. Most practitioners have followed these classifications to diagnose and apply treatment to their PMS patients.

Yang (1993) collated all the previous studies and discussions of PMS of both western and Chinese medicine, and proposed that the difference in the constitution of each individual leads to differences in PMS symptoms, thus treatment should be considered according to the following six different most common patterns:

1. Liver $Q i$ stagnation transforming into Heat;

2. Liver and Kidney Yin deficiency causing Liver Yang to flare up;

3. Liver and Kidney Yang deficiency giving rise to Water/ Dampness stagnation;

4. Exuberance of Heart Fire causing failure of the Heart and Kidney to communicate;

5. Qi and Blood deficiency causing disharmony between Ying Qi and Wei Qi;

6. Qi and Blood Stagnation causing Blockage in the Channels and Collaterals.

Gao and Chen (1994) categorised PMS according to 22 TCM patterns of disharmony (Table 3.1) from medical journal articles of China over a period of ten years. The most common patterns encountered were Liver $Q i$ stagnation 
and Liver and Kidney Yin deficiency causing Heat in the internal organs. The next common patterns were Spleen and Kidney Yang deficiency, Spleen and Heart deficiency, and Phlegm/Blood stasis blocking the meridians.

In research on the prevalence of TCM patterns in PMS, Qiao and colleagues (1997) found that the of Liver $Q i$ invasion, Liver $Q i$ stagnation, Liver Fire flaring up, and Heart and Spleen deficiency make up 95\% of PMS cases, of which the first and second patterns amount to $58.9 \%$ and $27.5 \%$ respectively. There are also other patterns, such as Liver and Kidney Yang deficiency, Spleen and Kidney Yang deficiency, and Heart and Kidney failing to communicate which however account for less than $1 \%$.

In a clinical research study (You 1997) using acupuncture to deal with PMS symptoms, 56 cases were grouped into two patterns, i.e. Liver $Q i$ stagnation (36 cases) and Spleen and Kidney Yang deficiency (20 cases). In another study (Ran 1997) on PMS using auricular point pressing as treatment method, 37 clients were grouped into three TCM patterns: Liver $Q i$ stagnation (18 cases),

Liver and Kidney Yin deficiency (11 cases) and Spleen and Kidney Yang deficiency (8 cases). Wang and colleagues (2003) used Chinese herbal medicine and medroxyprogestorone acetate together to treat PMS. The Chinese herbal medicine was applied according to pattern identification. 26 women in the treatment group were identified into four TCM patterns: Liver $Q i$ stagnation and Blood deficiency blocking Collaterals (15 cases); Spleen deficiency with Liver exuberance and Damp Heat being retained interiorly (4 cases); $Q i$ and 
Blood deficiency with Wind pathogen retaining (4 cases); and Liver stagnation transforming into Fire and Yin deficiency causing Yang to flare up (3 cases). The prevalence of TCM patterns in these studies, which were carried out by different professionals of Chinese medicine, showed similar occurrence and frequency to Gao and Chen's finding in 1994 and Qiao and colleagues' finding in 1997.

\section{Table 3.1 22 TCM Patterns of PMS}

\section{TCM Patterns of Disharmony}

1 Liver Qi stagnation

2 Liver Qi stagnation transforming into Fire

3 Disharmony between Liver and Spleen

$4 \quad$ Liver and Kidney Yin deficiency causing Liver Yang to flare up

$5 \quad$ Blood deficiency causing hyperactivity of Fire

6 Qi and Blood stagnation causing blockage in the Channels and Collaterals

7 Phlegm and Qi binding together / Blood Stasis causing obstruction

$8 \quad$ Spleen and Kidney Yang deficiency

9 Exuberance of Heart Fire causing failure of Heart and Kidney to communicate

10 Qi and Blood deficiency causing disharmony between Ying Qi and Wei Qi

11 Lung and Spleen Qi deficiency with Liver Fire flaring up

12 Dampness retaining in the Lung and Spleen

13 Sluggishness of Lung Qi

14 Yin deficiency causing hyperactivity of Fire that burns the Lung Collaterals

15 Kidney deficiency causing stagnation of Liver

16 Heart and Spleen deficiency

17 Liver and Kidney deficiency giving rise to Coldness that blocks the Liver Channels

18 Blood Stasis and Heat stagnating in the Chong and Ren Channels

19 Blood Stasis stagnating interior causing Liver Fire to flare up

20 Stomach and Kidney Yin deficiency causing the up rush of Qi

21 Yang deficiency and Qi insufficiency

22 Heat in the internal Organs 


\subsubsection{TCM Patterns according to Specific Symptoms of Peri-menstrual Disorders}

\section{$\underline{\text { Peri-menstrual Headache }}$}

Huang (2000) proposed that headache is a "branch-symptom" and the hypothesized disharmony between Liver, Spleen, Kidney, $Q i$ and Blood which is the root cause of peri-menstrual headache. Cases were grouped into 4 TCM patterns according to Pattern Identification and herbal treatment applied accordingly:

1. Yin deficiency and Liver exuberance ( 9 cases with a $89 \%$ effective rate);

2. $Q i$ and Blood stagnation (11 cases, with a $90 \%$ effective rate);

3. $Q i$ and Yin deficiency (6 cases, with a $83 \%$ effective rate); and

4. Yin-blood deficiency (4 cases, with a 100\% effective rate).

Zhong and Chen (2001) believed that "Disharmonies in Liver system are the primary causes of all women's diseases", and advocated a strategy to deal with peri-menstrual headache by treating only the Liver system. The most common Liver disharmonies in peri-menstrual headache are: Liver $Q i$ stagnation; Liver Blood deficiency; Liver and Kidney Yin deficiency; Liver $Q i$ stagnation giving rise to Blood Stasis blocking Collaterals, Liver Yang / Fire flaring up; and Phlegm-Dampness obstruction. Li and colleagues (2002) applied Liver coursing and nourishing methods to treat peri-menstrual headache in 40 cases with good result. The patients recovered in 28 cases, improved in 11 cases and had no effect in 1 case after 1-2 months of herbal treatment. Yang (2003) used 
Long Dan Xie Gan Tang, which is a traditional formula for TCM pattern of Liver Fire flaring up, to treat 30 cases of peri-menstrual headache with this pattern. A good result with $93.3 \%$ total effective rate was achieved.

\section{Peri-menstrual Breasts Distension and Pain}

Bi (2004), Gao (1999), Liang and colleagues (1996) and Wang and Xu (1984) all proposed that peri-menstrual breast distension and pain are mainly related to $Q i$ and Blood disharmony in the Liver meridians. Liver $Q i$ stagnation gives rise to Blood stasis which blocks the collaterals and results in water retention in the soft tissues, and this is the primary pattern.

\section{Peri-menstrual Oral Ulcer}

Wang and Zhang (1998) proposed to treat 48 cases of peri-menstrual oral ulcer in their study with herbal medicine according to 3 patterns: Yin deficiency causing exuberant Fire (31 cases, with recovery in 27 cases and improvement in 4 cases), Exuberant Heat in Stomach (12 cases, all with full recovery), and Spleen deficiency with Damp-Heat retention (5 cases, with recovery in 4 cases and improvement in 1 case).

Mo (1999) and Wu (2001) both recognised that peri-menstrual oral ulcer is due to Kidney deficiency in that Kidney-Water fails to cool the Fire in the Heart and Stomach, and the Fire flares up to their related external orifices. There were 26 cases of Yin deficiency causing exuberant Fire and 14 cases of Exuberant Heat in Stomach in Mo's study. A total effective rate of over 90\% 
were reported in both studies after Heat clearing herbs were prescribed 8 days before menstruation for 2-3 menstrual cycles.

According to these findings, peri-menstrual oral ulcer is mainly caused by Heat being retained in the body. The Heat can either be deficient or excess Heat. The most common pattern in this disorder is Kidney Yin deficiency.

\section{Peri-menstrual Oedema}

In a journal article, Yue (1994) discussed the etiology, classification and treatment method of premenstrual swelling in a Chinese medical perspective. The disorder was categorized, according to associated symptoms, into three TCM patterns: Liver Qi stagnation, Damp-Turbid blockage internally, and Blood Stasis stagnation. Treatment of the disorder was based on the identified pattern.

Liu (2000) hypothesised that Spleen and Kidney Yang deficiency which fails to drive away Water is the primary cause of peri-menstrual oedema, and that Liver $Q i$ stagnation, Blood stagnation and Blood deficiency during menstruation are the secondary causes. He proposed that the treatment for the disorder should deal with the weak/imbalanced systems of the body, i.e. the primary cause. A traditional herbal formula (Ling Gui Zhu Gan Tang) which has the action of warming the Spleen and Kidney Yang was applied to 46 cases of peri-menstrual oedema in his study. The success rate of this treatment was $86.7 \%$ in that the patients in 23 cases recovered fully, improved in 17 cases and had no effect in the remaining 6 cases. This result supported his hypothesis. 


\section{Peri-menstrual Emotional Abnormalities}

In a discussion paper of diagnosis and treatment of peri-menstrual emotional abnormalities, Wu \& Tan (2001) advocated that the clinical treatment for peri-menstrual emotional abnormalities should be categorised into 3

pattern-types: Liver $Q i$ stagnation, Phlegm-Fire flaring up, and Heart Blood insufficiency.

Both Liu and Wang (2002) and He and Li (2000) used the modified Gan Mai Da Zao Tang for the treatment of Heart Blood insufficiency in peri-menstrual emotional abnormalities. The herbal formula has the action to nourish the Heart and Liver and to calm the spirit. Liu and Wang (2002) applied the formula with psychotherapy to treat 30 cases of Heart Blood insufficiency of peri-menstrual emotional abnormalities in their study, whilst He and Li (2000) applied the formula with auricular point pressing to treat 30 women in their study. On the other hand, Hua (2000) used another formula that tonifies $Q i$ and Blood and nourish the Heart and Spleen to treat peri-menstrual emotional abnormalities. Gui Pi Tang was prescribed to 36 women in his study. The treatment strategies in these studies focus primarily on the Heart system which TCM believes it governs the spirit and the Blood systems. All these studies reported outstanding effect as their success rates were over $90 \%$.

\section{Peri-menstrual Fever/feverish Sensation}

Luo (1989) proposed that premenstrual fever is due to Liver $Q i$ stagnation, $Q i$ stagnation giving rise to Blood stagnation, and Blood stasis leading to Heat and 
manifesting as fever. Treatment using Liver coursing and Blood moving strategies was applied to 36 cases of premenstrual fever. A formula modified from Chai Hu Shu Gan Tang and Tao Hong Si Wu Tang was prescribed to these women before menstruation. The patients' temperature returned to normal before menstruation in 32 cases and conditions improved in 4 cases after treatment.

Zhao and colleagues (2000) applied herbal tablet Fu Ke Qian Jin Pian to treatment 55 cases of peri-menstrual fever/feverish sensations. In the study, 15 cases of Yin deficiency with internal Heat, 19 cases of $Q i$ and Blood deficiency, 8 cases of disharmony between Ying and Wei and 13 cases of Stasis and Heat stagnation were identified. The finding indicated that more than $50 \%$ of the women had deficient patterns. This finding is inconsistency with those of other studies where Liver Qi stagnation and Blood stasis are the most common patterns.

\subsubsection{TCM Patterns according to the Primary Causes}

\section{$\underline{\text { Liver System }}$}

Dong (2003) diagnosed cases according to 4 Liver disharmonies: Liver $Q i$ stagnation; Liver stagnation transforming into Fire; Stagnated Fire in the Liver meridians leading to reckless Blood; and Liver and Kidney Yin deficiency. 
Cai (1997) grouped women of PMS in 5 patterns clinically, which are Liver stagnation transforming into Fire; Liver stagnation and Phlegm accumulation; Liver stagnation consuming Blood; Liver stagnation attacking Spleen; and Liver stagnation damaging Kidney.

Huo (1995) treated PMS cases in 4 patterns: Liver stagnation; Liver stagnation transforming into Fire; Spleen deficiency leading to $Q i$ and Blood deficiency; and Yang $Q i$ deficiency causing blood stasis in the meridians.

\section{Yin Blood Deficiency}

Zhang and Chen (1998) concluded that PMS is either due to:-

1. $Q i$ and Blood disharmony which is caused either by a weak constitution or lack of replenishment after menstruation /pregnancy /delivery; or

2. Liver $Q i$ Stagnation which is caused by emotional disturbances.

Zhou and colleagues (2002) hypothesized that Yin Blood deficiency with Wind invasion is the primary pattern of menstrual related urticaria.

Lin (2000) hypothesized that Coldness and Blood stagnation is the chief pattern in peri-menstrual headache, which occurs mainly in women with Yin-Blood deficiency in that $Q i$ and Blood fail to protect the Ying and Wei and open to Cold invasion. 


\subsubsection{Summary}

The present TCM classification of patterns for PMS are extremely repetitious and tedious. Variation in the approach adopted by different researchers leads to considerable confusion. Irrespective of whether pattern identification is carried out based on the whole syndrome, specific symptoms, or the root causes, the ultimate objective is to identify the chief complaints and categorise them into the appropriate patterns or patterns of disharmony. The present process and procedures for pattern classification should therefore be rationalized and simplified so as to facilitate more effective application for the benefit of future research and treatment of PMS.

\subsection{TCM Treatment Approaches in Premenstrual Syndrome}

Prior to the 1960's, in contemporary Chinese medicine relatively little was known about PMS as a specific syndrome/disorder and treatment was generally based on identification of individual premenstrual complaints. Simple research began in the 1960's when PMS was recognised and described as a syndrome by Chinese medical professionals, and as a result a new topic "peri-menstrual disorders" was included in Chinese medical textbooks in the 1970's. Further research continued in the 1980's leading to various Chinese medical treatment strategies for PMS. Chinese herbal medicine, which has been the principal means for internal treatment in TCM (Tian and Zou 2000), includes treatment for the whole syndrome, the primary symptoms, the organ systems and 
treatment according to menstrual cycle phases. Other therapies are available such as acupuncture, auricular point pressing, massage, and external application.

\subsubsection{Treatment for the Syndrome of PMS}

In the early 1980's, two large pioneering TCM studies described the basic patterns for PMS. Xu (1982) treated 170 cases of PMS by applying herbal medicine according to TCM pattern identification. The PMS sufferers were divided into 4 pattern types and treatment was applied accordingly. Modified Chai Hu Shu Gan Tang was applied to 117 cases of Liver Qi stagnation; herbs Shang Di, Mai Dong, Mu Dan Pi, Shan Zhi Zi, Huang Qin, Huang Lian and Da Huang were applied to 35 cases of Exuberant Internal Heat; modified formulae Zhen Wu Tang, Shen Ling Bai Zhu San, Wu Ling San, and /or Yu Ping Feng San were applied to 16 cases of Yang deficiency with Insufficient $Q i$; and herbs Zhi Shi, Da Huang, Tao Ren, Mu Dan Pi, Shan Zhi Zi, Yuan Zhi, Dang Gui, Nan Xing, Gou Teng, Jiang Can, and Xuan Xie were applied to 2 cases of Blood and Phlegm blocking Collaterals. The results indicated that full recovery was achieved in 104 cases, treatment was effective in 55 cases whereas no effect whatsoever was observed in 11 cases. The success rate was $93.53 \%$.

In another study in 1982, Cheng and colleagues applied treatment based on pattern identification for 102 cases of premenstrual tension syndrome. 100\% 
success rate with 81 cases of full recovery was reported. The clients were grouped into six TCM patterns:-

- Liver stagnation with Kidney deficiency (30 cases): Yi Shen Jie Yu Tang (Shu Di, Shan Yao, Chai Hu, Dang Gui, Lu Jia Pian, Xian Ling Pi, Tu Si Zi, Xu Duan, Xiang Fu, Ba Yue Zha, Fu Shen and Mei Gui Hua) were applied.

- Disharmony between Liver and Spleen (24 cases): modified Xiao Yao San was applied

- $\quad$ Liver $Q i$ stagnation (7 cases): modified Chai Hu Shu Gan Tang was applied.

- Liver stagnation transforming into Heat (17 cases): modified Dan Zhi Xiao Yao San was applied.

- Yin deficiency with hyperactive Liver (16 cases): modified Qi Jue Di Huang Tang was applied.

- Spleen and Kidney Yang deficiency (8 cases): modified You Gui Tang, Bu Zhong Yi Qi Tang, Li Zhong Tang, and/or Juan Bi He Xue Tong Luo Tang were applied.

However, these were uncontrolled studies and their results were measured with subjective methods only, so the findings must be viewed with caution and perhaps some skepticism.

In recent years, Chen (2001) applied herbal treatment to 188 cases of PMS. The subjects were divided into four TCM pattern groups i.e. Liver $Q i$ stagnation; Exuberant Heat in the Heart and Liver; Liver and Kidney Yin deficiency; and Spleen and Kidney Yang deficiency. Formulae used were:- modified Chai Hu 
Shu Gan San, Long Dan Xie Gan Tang, Yi Guan Jian, and Shen Ling Bai Zhu San with Ling Gui Zhu Gan Tang.

Zhang (2004) considered that Liver $Q i$ stagnation with Heat is the primary cause of PMS, and applied a traditional Liver coursing and clearing formula: modified Dan Zhi Xiao Yao San as the basic formula for the treatment of PMS in her study. Additional herbs were added according to different TCM patterns:

- Liver and Kidney Yin deficiency/ Liver Yang flaring up: Nu Zhen Zi, Han Lian Cao and Jue Hua were added

- Yin deficiency causing hyperactive of Fire/ Heart and Kidney failing to communicate: Chao Zao Ren, Sha Shen and Mai Dong were added

- Spleen and Kidney Yang deficiency/ Deficient Fire flaring up recklessly: Bai He, and Sheng Di were added

- Heart and Spleen both deficiency: Dang Shen, Chao Zao Ren and Bai He were added.

Outstanding results were also achieved in these two studies and no side effect was observed. Again, these studies were uncontrolled study and only subjective measures were used. 


\subsubsection{Treatment for Specific Symptoms of PMS}

A number of TCM researchers categorised PMS according to the dominant complaints of the sufferers in line with the classic texts. Some Chinese medical practitioners follow this approach when applying treatment for PMS.

\section{Peri-menstrual Breast Distending Pain}

Gao (1999), Li (2000), Yang (2000),Wang and Zheng (2002), Zhang (2003a), Tian and colleagues (2003), Bi (2004), and Zhao and colleagues (2005) all share the same view that peri-menstrual breast distending pain has a very closed relation with the Liver system. However, they have different opinions on the proposed treatment for PMS which is based on the strategies of Liver coursing, Qi regulating, Blood moving and Collaterals unblocking. The herbs commonly used for this disorder are Chai Hu, Bai Shao, Xiang Fu, Qing Pi, Ju Ye, Wang Bu Liu Xing, and/or Lu Lu Tong.

Bi (2004) prescribed a self-designed formula Shu Gan Tong Luo Tang for 50 women reporting peri-menstrual distending pain. Outstanding effect was shown after 2 months of treatment in that there were 40 cases with fully recovery, 8 cases with improvement, 2 cases with no effect and a total effective rate of $96 \%$. The ingredients of the formula include Chai Hu, Chuan Xiong, Ju Ye, Qing Pi, Gua Lou Pi, Chuan Lian Zi, Xiang Fu, Dang Gui, Chi Shao and Si Gua Luo. Bi acknowledged that following herbs:- Chai Hu, Ju Ye, Qing Pi, Gua Lou Pi, Chuan Lian Zi, Xiang Fu, and Si Gua Luo have the action to course Liver, regulate $Q i$, and unblock collaterals, and herbs: Chuan Xiong, Dang Gui, and 
Chi Shao have the action to move blood and unblock Collaterals. All these herbs when working together enable the $Q i$ and Blood of the Liver meridians to move smoothly without any blockage, and the distension pain of the breasts will vanish. The herbal medicine was prescribed to participants premenstrually for two menstrual cycles.

Wang and Zheng (2002) used patent herbal pills: Xiao Yao Wan to treat 30 cases of premenstrual breast distending pain, and Zhang (2003a) also applied Xiao Yao San with Xia Ku Cao for sufferers of premenstrual breasts distension pain with a satisfactory result. Both studies reported a total effective rate of over $96 \%$. Since that the breasts are located along the Liver meridians, that pain is caused by stagnation, and that Xiao Yao San is a traditional formula to deal with Liver $Q i$ stagnation, they acknowledged that this simple traditional formula, even in the forms of pills, can be used to treat cases of premenstrual breast distension pain effectively.

\section{Peri-menstrual Headache}

Most TCM practitioners agree that deficiency of Liver blood is the primary cause of peri-menstrual headache, and that the blockage in the collaterals by $Q i$, Blood, phlegm or stasis is the secondary cause. The key treatment strategy is to unblock the Collaterals and reduce pain by nourishing and moving Blood. Additional herbs are added according to TCM patterns, the location of the headache, and the traditional TCM action and/or modern pharmacology of the herb to enhance the treatment effect. 
Sun (2003) and Mu (2004) both applied the strategies of nourishing Blood and Liver, extinguishing Wind, and unblocking Collaterals to treat premenstrual headache. Sun (2003) prescribed a self-designed formula which included Dang Gui, Bai Shao, Chuan Xiong, Ju Hua, Man Jing Zi, Yu Jin, Shi Chang Pu, Sheng Di Huang, Gui Ban, Tian Dong, Di Long, Dang Shen, Huang Qi, Xuan Shen, Wu Gong, Quan Xie, and Gan Cao to 48 women in the treatment group. The other 48 women in the control group received ergotamine and caffeine tablets, aspirin, and flunarizine capsules. There was a statistically significant difference $(\mathrm{p}<.01)$ favouring the herbal treatment in the total effective rate between the two groups, and no adverse effects were reported. Mu (2004) also prescribed a self-designed formula to treat 68 cases of premenstrual headache. The formula which contains herbs: Dang Gui, Ju Hua, Sheng Di, Gou Teng, Di Long, Dan Cao, Bai Shao, Shan Zhu Yu, Chuan Xiong, Chai Hu, Long Gu, Mu $L i, N i u X i$, and $W u$ Gong was prescribed 10 days before menstruation for three months. A total effective rate of $97.2 \%$ was achieved, and also no adverse effect was reported.

Cao and Qin (2001) applied a self-designed Chuan Xiong Tang with adjuvant herbs according to the location of the headache to treat 105 cases of premenstrual headache. Bai Zhi was added for frontal headache, Ma Huang and Ge Gen were added for headache radiating to the back of the neck, Chai $\mathrm{Hu}$ was added for headache on the sides of the head, and Man Jing Zi and Wu Yao were added for parietal headache. 
Huang (2000a) applied treatment according to pattern identification to 30 women of peri-menstrual headache. Participants were divided into four groups by TCM patterns. Four different self-designed formulas were prescribed to women in these groups for 2 weeks. However, Wu Yao, Yan Hu Suo, and Bai Shao were included in all four formulas and these three herbs have the action to raise the pain threshold (cited in $\mathrm{Mu}, 2003$ ).

Cai and Wang (2005) used a formula named Xiong Zhi Shi Gao Tang to treat 50 cases of peri-menstrual headache. The formula contains herbs: Chuan Xiong, Bai Zhi, Gao Ben, Jiang Can, Shi Gao, Ju Hua, Dan Shen, Bai Shao, and Xi Xin. The application of these herbs to treat headache was in accordance with both the TCM and modern pharmacological action of the herbs. Cai and Wang pointed out that Dan Shen and Chuan Xiong have a vasodilative mechanism that may across the blood brain barrier, so they can improve the circulation, have a sedative effect and can alleviate headache. Bai Zhi stimulates the vasomotor and vasovagal nerves, and has an analgesia effect. Хi Хin, Ји Ниа, Bai Shao and Gao Ben all have the actions of sedation, analgesia, and anti-spasm. Jiang Can has an action of hypnosis. All these herbs together may regulate the contraction and relaxation function of the blood vessels.

\section{Peri-menstrual Diarrhoea}

Li (2000), Zhang and Li (2004) concluded that the exhausting use of body and mind may give rise to a weak Spleen and Stomach. The Spleen is further weakened through lack of nourishment and being deficient before and during 
menstruation when more blood is drawn to the lower body. This is the primary cause of peri-menstrual diarrhoea. Herbs that have the action of tonifying and warming the Spleen and Kidney, together with astringent herbs should be used to treat this condition. Formula Shen Ling Bai Zhu San was chosen and applied to participants of their studies with good results.

Zhang (2003b), however, recognized that Spleen and Kidney Yang deficiency was the primary cause of peri-menstrual diarrhea, and applied a traditional Spleen and Kidney tonic, the modified You Gui Wan, as the basic formula to treat 19 women of peri-menstrual diarrhoea. The modified You Gui Wan which contains Shou Di, Shan Yao, Gou Qi Zi, Lu Jiao Jiao, Tu Si Zi, Du Zhong, Shan Zhu Yu, Dang Gui, Rou Gui, and Zhi Fu Zi was prescribed 10 days for two menstrual cycles to those women in his study.

\section{Peri-menstrual Swelling}

TCM practitioners have different opinions and may apply different strategies to deal with cases of peri-menstrual swelling. However, most of them include in their formulas such herbs as Bai Zhu and Fu Ling which have the action of tonifying $Q i$, strengthening Spleen, removing Dampness and promoting diuresis.

Both Liu (2000) and Xu (2003) used the traditional Ling Gui Zhu Gan Tang as the basic formula to treat women with peri-menstrual swelling in their studies. Ling Gui Zhu Gan Tang has the action of warming the Kidney, strengthening the Spleen, transforming Dampness and promoting diuresis. Liu (2000) applied 
Ling Gui Zhu Gan Tang with adjuvant herbs to address different patterns in 46 cases of peri-menstrual swelling with a success rate of $86.7 \%$. The herbal formula was prescribed 7 days before menstruation and stopped once menses started. Xu (2003) prescribed Ling Gui Zhu Gan Tang with vitamin B6 to participants in the treatment group, and a diuretic-dihydrocholorothiazide, with Vitamin B1 and B6 to the control group. The medicines were to be taken 7 days before menstruation and stopped when menses started for 3 menstrual cycles. There was significant difference $(\mathrm{p}<.05)$ in the total effective rate between the two groups. The treatment group had a total effective rate of $93.3 \%$ and the control group $65.5 \%$.

Zhang (1995) believed that the blockage of stasis was the major cause of swelling before menstruation and used modified Dang Gui Shao Yao Tang to treat 15 cases of premenstrual swelling in a study. Zhang believed that the three main herbs i.e. Dang Gui, Shao Yao and Chuan Xiong had the actions to nourish and move the Liver blood and to address the disorder.

\section{Peri-menstrual Emotional Change}

Wu and Tan (2002) concluded that peri-menstrual emotional changes were primarily caused by the weak Kidney system of women. When there is not enough Yin during the peri-menstrual period to nourish the Liver, the Liver becomes fired, and further affects the Heart and Spleen system. The most common TCM patterns of the disorder are Liver Qi stagnation, Phlegm or Fire 
in Liver and Heart, and insufficient Heart Blood, and the most important treatment strategies are to nourish the Heart and to calm the Shen (spirit).

Liu and Huang (2002) combined herbal medicine, which was modified from Gan Mai Da Zao Tang and Yang Xin Tang, and psychotherapy to treat 30 cases of peri-menstrual emotional changes with Heart Blood insufficiency. The patients recovered in 27 cases and improved in 3 cases.

Hua (2000) applied Gui Pi Tang to nourish the Heart and to strengthen the Spleen in treating peri-menstrual emotional changes. Six packs of the herbal formula were prescribed to women in the study before menstruation for three cycles. The women recovered in 29 cases (80.6\%), improved in 6 cases (16.7\%) and no effect in 1 case $(2.8 \%)$.

\section{Peri-menstrual Oral Ulcer}

Wu (2001), Gao and Chen (2001) admitted that peri-menstrual oral ulcer had a very close relationship with Heart Fire and Stomach Heat of the body. They applied treatment methods aiming at nourishing Yin, clearing Stomach and purging Fire to deal with peri-menstrual oral ulcer. Wu (2001) used a self-designed formula named Qin Lian Er Di Tang to treat 40 cases of peri-menstrual oral ulcer in a study. The herbal formula has ingredients including Huang Lian, Huang Qin, Zhi Mu, Dang Gui, Shang Di, Shou Di, Zi Cao, Shan Zhu Yu, Bai Shao, Nu Zhen Zi, Mu Li, Bao Fu Zi, and Gan Cao. The herbal formula was prescribed six days before menstruation for 6 months. Gao and Chen (2001) also used a self-designed herbal formula named Qin Zhi Yin to 
treat 42 cases of peri-menstrual oral ulcer. The herbs: Huang Qin, Zhi Mu, Zhu Ye, Sheng Di, Shi Hu, Zhen Zhu Fen, Rou Gui Fen and Gan Cao were prescribed to the treatment group, and an antibacterial lozenge was prescribed to the control group of the study. There was significant difference $(\mathrm{p}<.01)$ in the treatment outcome between the two groups in that herbal medicine had a better effect.

Wang (2004) applied a treatment strategy that addresses both Liver and Kidney. It also tonifies both Yin and Yang for the treatment of peri-menstrual oral ulcer with remarkable effect. Wang applied some sweet and nourishing herbs to deal with the Liver and Kidney Yin, and some warming Yang herbs simultaneously to guide the Yin fire back to its original place. Herbal medicine: Xuan Shen, Mai Dong, Rou Gui, Chuan Lian Zi, Sheng Di, Gou Qi Zi, Fu Zi, DaiZhe Shi, Yu Zhu, Dang Gui, Niu Xi and Shou Wu were used.

\section{$\underline{\text { Peri-menstrual Fever/feverish Sensation }}$}

In Zhou and Cao's paper (2000), they identified that the etiology of peri-menstrual fever/feverish sensation can be concluded as: exterior invasion, Liver stagnation transforming into heat, insufficient Zhong $Q i$, and/or stasis stagnation transforming into Heat. However, in most cases of peri-menstrual fever/feverish sensation, there may be several causes intertwining and consequent upon one another giving rise to the disorder. The best treatment strategy is to use a harmonizing and releasing method. In the case of exterior invasion, Gui Zhi Si Wu Tang or Gui Zhi Chai Hu Tang can be applied. For Liver stagnation, Xiao Chai Hu Tang or Dan Zhi Xiao Yao San can be applied. 
For insufficient Zhong Qi, modified Bu Zhong Yi Qi Tang can be applied. For stasis stagnation transforming into Heat, Tao Hong Si Wu Tang or Tao He Cheng Qi Tang plus Xiao Chai Hu Tang can be applied.

Huang (2000b) and Lin (2005) applied the classic harmonizing formula: Xiao Chai Hu Tang to treat peri-menstrual fever/feverish sensation irrespective of any patterns. Huang (2000b) used the traditional Xiao Chai Hu Tang with additional herbs; Dang Gui, Chi Bai Shao, Chuan Xiong, Tao Ren, Qian Cao and Dan Pi. Lin (2005) used the traditional Xiao Chai Hu Tang with different adjuvant herbs according to different patterns.

\subsubsection{Treatment for the Organ Systems}

According to TCM, it is believed that the excess or deficiency of different organ systems of the body may give rise to different symptoms of PMS. Practitioners apply different treatment strategies to correct the imbalance of the organ systems.

Gao (2005) recognized that PMS is a complex and multi-symptom disease, so he advocated that treatment methods for the disease should be varied and flexible. In terms of the relation between PMS symptoms and the five organs i.e. Liver, Kidney, Heart, Spleen and Lung, He acknowledged that treatment for PMS should fall into five categories. Modified Xiao Yao San was used to deal with Liver $Q i$ stagnation transforming into Heat; modified $E r$ Zhi Wan for 
Liver and Kidney Yin deficiency with hyperactive Kidney Yang; modified Sheng Mai San plus Gan Mai Da Zao Tang for Yin deficiency with exuberant Heat causing failure of the Heart and Kidney to communicate; modified Ling Gui Zhu Gan Tang for the Spleen which has lost its function of transportation giving rise to retention of fluid and dampness in the body; and Ye Ju Hua, Lian Qiao, Bo He, Jin Yin Hua, Lu Gen, Huang Qin, Qing Dai, and Er Cha for Heat in the Lung and Stomach with Ying-Wei disharmony.

Most TCM researchers agree that treating the Liver is the primary strategy for PMS. Dong (2003) concluded that there are four methods for regulating the Liver with traditional formulas in the treatment of PMS: - 1) Coursing the Liver by modified Xiao Yao San; 2) Calming the Liver by modified Ling Yang Gou Teng Tang and Qing Gan Tang; 3)Clearing the Liver by modified Long Dan Xie Gan Tang; and 4) Nourishing the Liver by modified Qing Jing Tang. Formula should be applied according to the state of the Liver.

Some researchers use Liver coursing formulas for PMS with an effective rate $>$ 90\%. Wang (2000) applied herbs: Chua Hu, Dang Gui, Bai Shao, Xiang Fu, Yu Jin, Bai Zhu, Fu Ling, Yi Mu Cao, and Gan Cao as the basic formula, and added additional herbs based on the symptoms to 62 cases of PMS sufferers. Tang (2005) applied self-prescribed Shu Gan Tiao Jing Tang which was composed of Dang Gui, Chuan Xiong, Cang Zhu, Shan Zhi Zi, Shen Qu, Zhi Qiao, Shou Di, Yu Jin, Chen Pi and Gan Cao. 
In contrast, while applying treatment for PMS, some practitioners specifically target other organ systems. Zhou (2002) recognized that Kidney is the mother of Liver. In order to nourish the Kidney-water so as to enrich the Liver-wood, Zhou prescribed Liu Wei Di Huang Tang after menstruation to nourish Kidney Yin, and Xiao Yao San before menstruation to course the Liver $Q i$ to 96 cases of PMS sufferers in the treatment group. Women in the control group only received Xiao Yao San before menstruation. The result indicated a significant difference $(p<.01)$ between the two groups in that the treatment group had a better outcome. Zhang (2003b) believed that Kidney and Spleen yang deficiency is the most common cause for peri-menstrual diarrhoea, and prescribed You Gui Wan to warm the Kidney Yang for 19 cases of peri-menstrual diarrhoea.

Liu (2000) considered that the pathogenesis of peri-menstrual swelling is related to the Spleen system. Ling Gui Zhu Gan Tang was prescribed to 46 women suffering peri-menstrual swelling in Liu's study. Liu and Huang (2002) believed that insufficient Heart Blood is the most common pattern in peri-menstrual emotional changes. They used a formula, which is an integration of Gan Mai Da Zao Tang and Yang Xin Tang, for 30 cases in their research to study the Chinese herbal effect on peri-menstrual emotional changes. Since Yin Blood insufficiency is another underlying cause that gives rise to PMS, Zhang (2004) treated 36 cases of PMS using a traditional formula for harmonising Blood and regulating Liver, namely modified Dang Gui Shao Yao San. Herbs include Bai Shao, Dang Gui, Chuan Xiong, Bai Zhu, Ze Xie, Fu Ling, Yu Jin, Dan Shen, Tu Si Zi, Mai Ya, and Gan Cao. All these studies which covered 
different symptoms, proposed etiologies, TCM patterns and herbal formulas, reported an effective rate of over $86 \%$. This should provide evidence that Pattern Identification of TCM is a right diagnostic tool for syndrome disorders such as PMS.

\subsubsection{Treatment according to Menstrual Cycle Phases}

In 1982, Cheng and colleagues advocated to apply TCM menstrual cycle therapy for the treatment of PMS. The basis for this method is still the application of the entire range of TCM including theories, pattern identification, disease identification and Chinese herbal medicine. However, by integrating the theory of hormonal phase in a menstrual cycle of western medicine, a treatment plan including formulas with different actions and herbs was designed for the various phases of a menstrual cycle. The principles of the plan are: Follicular phase- nourishing Kidney, tonifying Blood, and assisting Kidney $Q i$; Pre-ovulatory phase- also nourishing Kidney and tonifying Blood, but adding herbs to assist Yang and to move $Q i$ and Blood; Luteal phase- assisting the Kidney Yang and regulating the balance between Yin and Yang; Late Luteal phase and Menstruation: Moving Qi and Blood.

Chen and Chai (2001) applied TCM menstrual cycle therapy to treat PMS.

During the follicular phase, Cu Pai Luan Tang (ingredients: Shou Di, Dan Shen, Shan Yao, Bai Shao, Xiang Fu, He Shou Wu, Bai Zhu, Tu Si Zi, and Rou Cong Rong) was prescribed for 7-10 days. During the ovulation time, Pai Luan Tang 
(ingredients: Dan Shen, Tao Ren, Chuan Niu Xi, Dang Gui, Lu Lu Tong, Che Qian Zi, Xiang Fu and Zhi Qiao) was prescribed for 2-3 days. During the luteal phase, Yi Qi Zi Shen Tang (ingredients including Huang Qi, Bai Zhu, Shan Yao, Huang Jing, Sang Ji Sheng, Xu Duan, Nu Zhen Zi, Han Lian Cao, Tu Si Zi, and Yin Yang Huo) was prescribed for the PMS sufferers until the commencement of their next menstruation. This method showed an outstanding effect in that there were 52 cases $(81.3 \%)$ with full recovery, 10 cases $(15.6 \%)$ with improvement, 2 cases (3.1\%) with no effect and a total effective rate of $98.44 \%$.

Zhang (2003c) also applied herbal treatment for PMS in line with menstrual cycle. However, Zhang applied different herbs to deal with the symptoms before menstruation and the primary causes after menstruation. In the study, herbal formula Dan Zhi Xiao Yao San was prescribed before menstruation to resolve the stagnation, which is believed to be the cause of most PMS symptoms. Also, herbal formula Yi Quan Jian was prescribed after menstruation to nourish the Kidney and enrich the Liver.

\subsubsection{Treatment with Integrated Traditional Chinese and Western Medicines}

The idea of integrating traditional Chinese medicine and western medicine was conceived around the middle of the 20th century (Shin 1999). It was considered that better results could be achieved by combining and thereby exploiting the 
uniqueness of medical treatment in TCM and new technologies/knowledge in modern western medicine. From the 1990s, many researchers have explored the possibility of treating PMS with integrated TCM and western medicine.

In 2001, Kou and Wang evaluated the treatment effectiveness of integrated TCM and western medicine on 52 PMS women. The TCM treatment was based on a four-herbs formula Da Ying Tang (San Leng, Da Huang, Dan Pi, and Chi Zhu) with additional herbs for different TCM patterns. The western medicines were Alprazolam and Oryzanol. The combined use of Chinese herbal formula, antianxiolytic and autonomic drugs gave a result of $76.9 \%$ total effective rate.

Li and colleagues (2002), Zhang and Sun (2003), and Wang and colleagues (2003) compared the treatment effect of integrated traditional Chinese and western medicine with western medicine. Medroxyprogestorone acetate was prescribed for the control groups in these studies. For the integrated TCM and western medicine groups, $\mathrm{Li}$ and colleagues (2002) prescribed medroxyprogestorone acetate with herbal formula Jie Yu Xiao Zhang Jian (herbal ingredients: Chai Hu, Long Dan Cao, Sheng Di, Dan Pi, Yan Hu Suo, Xiang Fu, Bai He Hua, Yuan Zhi, Dang Gui, Liu Ji Nu, Yi Mu Cao, Mai Ya, Bai Shao, Chuan Xiong, Fu Ling, Bai Zhu and Zhi Gan Cao); Zhang and Sun (2003) prescribed medroxyprogestorone acetate with a self designed formula Shu Jin Tang (herbal ingredients: Xiang Fu, Chai Hu, Dang Gui, Chuan Xiong, Huai Niu Xi, Tao Ren, Shou Di, Shan Zhu Yu, Bai Shao, Sheng Long Gu, Sheng Mu $L i$, and $Y i \mathrm{Mu} C a o$ ); and Wang and colleagues (2003) prescribed medroxyprogestorone acetate with herbal medicine based on TCM patterns. 
There were significant differences $(\mathrm{p}<.05)$ between the integrated groups and the western medicine groups in all three studies, favouring the integrated medical treatment.

Shao and Guo (2005) used integrated TCM and western medicine to treat 98 cases of PMS with an outstanding result. A basic TCM herbal formula of Dang Gui, Chai Hu, Sheng Bai Shao,Yu Jin, Fu Ling, Jiao Bai Zhu, Xiang Fu, Ze Lan, Chao Suan Zao ren, Sheng Long Gu, Sheng Mu Li, Chen Pi, and Gan Cao with additional herbs for different symptoms, and western medicine comprising ibuprofen, diazepam and spironolactone were prescribed 7-14 days before menstruation for 3 menstrual cycles.

\subsubsection{Types of TCM Herbal Formulas and Herbs}

In recent years, many clinical practitioners have adopted relevant formulas prescribed in TCM medical classics as the basis for treating PMS, with additional herbs to address different symptoms, whilst others have created their own formulas through their clinical experiences.

Most TCM practitioners apply herbal formulas and/or herbs either following individual symptoms, pattern identification, disease identification or modern pharmacological research. The basic types of TCM herbal formulas and herbs for the treatment of PMS can be grouped as follows: 


\section{Liver Coursing}

The most commonly used herbs for this method are Chai $\mathrm{Hu}$, Dan Pi, Xiang Fu, Qing Pi, Yu Jin, Dang Gui, and Bai Shao. Most of these herbs, according to western pharmacology (Chang \& But 1996; Huang 1994), have sedative, analgesic, antipyretic, spasmolytic, anti-inflammatory and anti-bacterial effects (Table 3.2).

The most common formulas for this method are Xiao Yao San, Dan Zhi Xiao Yao San, Chai Hu Shu Gan San, and Shao Chai Hu Tang.

Xiao Yao San is a formula originated from Tai Ping Hui Min He Ji Ju Fang of the Song Dynasty (A.D. 960-1279). It has the action to course the Liver, to release stagnation, to strengthen the Spleen and to harmonise Blood. Most practitioners agree that Xiao Yao San is the most appropriate classic formula for treating PMS. In 1999, Yan applied modified Xiao Yao San ( Dang Gui, Bai Shao, Shao Chua Hu, Fu Ling, Shao Bai Shao, Xu Duan, Gou Teng, Dan Pi, Shan Zhi Zi, Xiang Fu, Qing Pi, Chen Pi and Jing Jie) to treat PMS caused by Liver stagnation transforming into Heat. The result indicates that there were significant differences $(\mathrm{p}<.01)$ in the ratios between the progesterone and estradiol before and after treatment (before treatment $\bar{x} \pm s=69.2 \pm 31.4$, after treatment $=118.1 \pm 5.02)$, and the prolactin level (before treatment $=53.4 \pm$ $27.1 \mu \mathrm{g} / \mathrm{L}$, after treatment $=19.7 \pm 14.3 \mu \mathrm{g} / \mathrm{L})$

Wang and Cui (1997), Yang and Zhao (2003), Fu (2003), Gao (2003), Wang and Zhang (2003), and Yue and Chen (2005) used Modified Xiao Yao San to 
treat PMS in their studies. Yang and Zhao (2003) prescribed the modified Xiao Yao San with herbs: Chua Hu, Dang Gui, Bai Shao, Fu Ling, Yu Jin, Ju He, Dan Bai Zhu, Bo He, Qing Pi and Gan Cao to treat 40 cases of PMS. Additional herbs Ju Ye, Wang Bu Liu Xing, Xia Ku Cao, Chuan Lian Zi, Quan Gua Lou, and Mai Ya were added for women with sore and distended breasts. Dan Pi, Zhi Zi, Yu Jin, Tian Hua Fen, and Long Dan Cao were added for bitter mouth, dry throat, emotional disturbance, a tongue with red sides and wiry, slippery and rapid pulse. Gou Teng, Ci Ji Li, and Di Long were added for headache. Suan Zao Ren, Da Zao, Long Chi, and Ye Jiao Teng were added for insomnia. Shan Yao and Da Zao were added for Liver stagnation with Spleen deficiency. Yue and Chen (2005) also applied modified Xiao Yao San to evaluate the efficacy and clinical significance of the formula to PMS sufferers. A herbal formula with herbs Chua Hu, Long Dan Cao, Sheng Di, Dan Pi, Yan Hu Suo, Bai He Hua, Yuan Zhi, Dang Gui, Yi Mu Cao, Mai Ya, Bai Shao, Chuan Xiong, Fu Ling, Bai Zhu, and Zhi Gan Cao was prescribed 14 days before menstruation for 10 days for 3 menstrual cycles to 300 cases of PMS women in the treatment group. The women in the control group (200 cases) were prescribed with medroxyprogesterone acetate and Vitamin B6. There was significant difference $(\mathrm{p}<.01)$ between the two groups, favouring the herbal formula.

Li and colleagues (2003) applied a patent herbal mixture Jing Qian Shu Yi, and Huang (1999) applied a patent formula Kun Yue Zhu to treat PMS with satisfactory result. Both formulas are modified from Xiao Yao San. 
Whilst Xiao Yao San has been used to treat PMS, some practitioners have also used it to treat peri-menstrual symptoms. Zhong and Chen (2001) applied Xiao Yao San to treat peri-menstrual headache caused by Liver Qi stagnation. Wang and Zheng (2002) used Xiao Yao Wan to treat peri-menstrual breasts pain and distension, and Zhang (2003a) prescribed Xiao Yao San with Xia Ku Cao for peri-menstrual breasts pain and distension.

Other Liver coursing formulas have also been used by practitioners to deal with PMS. Zhang (2004a) and Chen (2005) applied the modified Dan Zhi Xiao Yao San for PMS in their studies. Both studies had a total effective rate of over $98 \%$. Chen (2001) applied Chai Hu Shu Gan Tang to treat PMS women with Liver Qi stagnation. Chen and Chen (1996) used Chai Hu Shu Gan Tang and Sheng Hua Tang to treat peri-menstrual emotional changes. Huang (2000b) and Lin (2005) prescribed modified Xiao Chai Hu Tang for peri- menstrual feverish sensation sufferers. Yi (2004) applied Xiao Chai Hu Tang combined with Hei Xiao Yao San to treat peri-menstrual emotional disturbances.

\section{$\underline{\text { Liver Clearing and Heat Purging }}$}

Yuan (2002) and Dong (2003) both advocated the use of Long Dan Xie Gan Tang to deal with Liver Fire in PMS. Jiang (2003) applied modified Long Dan Xie Gan Tang to treat 84 cases of pre-menstrual acne. Qiu (2003) prescribed modified Long Dan Xie Gan Tang to alleviate pre-menstrual distending headache in a study. Total effective rates of over $90 \%$ were reported in both studies. 
Herbal ingredients in the formula are Long Dan Cao, Sheng Di Huang, Dang Gui, Huang Qin, Zhi Zi, Chai Hu, Ze Xie, Dan Shen, Yi Yi Ren, Bai Xian Pi, Che Qian Zi, Mu Tong and Gan Cao. Most of these herbs, according to western pharmacology (Chang \& But 1996; Huang 1994), have sedative, hypotensive, diuretic, analgesic, antipyretic, hypothermic, liver protective, anti-inflammatory and anti-bacterial effects (Table 3.3 ).

\section{Liver and Kidney Nourishing}

The commonly used herbs for this method are Dang Shen, Fu Ling, Bai Zhu, Che Qian Zi, Gan Cao, Gu Ya, Mai Ya, Cang Zhu and, Ze Xie. Most of these herbs, according to western pharmacology (Chang \& But 1996; Huang 1994), have hypoglycemic and anti-bacterial effects (Table 3.4).

The common formulas for this treatment method for PMS are Yi Guan Jian, Shen Qi Wan, Qi Ju Di Huang Wan, You Gui Wan, Er Xian tang, Bai He Zhi Mu Di Huang Tang etc.

Chen (2001), Zhong and Chen (2001) applied Yi Guan Jian for PMS sufferers with Liver and Kidney Yin deficiency. Chen (2001) prescribed Yi Guan Jian plus Sang Ji Sheng, Sang Shen, and Bai Shao to clients of her study, and added Dan Pi, Yu Jin and Zhi Zi for cases with anxiety or feverish sensation, and Man Jing Zi and Bai Ji Li for cases with headache. Zhong and Chen (2001) used 
modified Yi Guan Jian to treat peri-menstrual headache with Liver and Kidney Yin deficiency.

Cao and colleagues (2000) applied Shen Qi Wan and You Gui Wan combination for pattern of Kidney Yang deficiency in PMS, whilst Zhang (2003b) used modified You Gui Wan to treat 19 cases of peri-menstrual diarrhoea.

Zhou (2002) applied the strategy of nourishing the Kidney to enrich the Liver to treat women with peri-menstrual distending pain in their breasts. Liu Wei Di Huang Wan was prescribed after menstruation and Xiao Yao Wan was prescribed before menstruation for the treatment group. The control group only received Xiao Yao Wan premenstrually. There was a significant difference ( $\mathrm{p}$ $<.01)$ between the two groups in that the treatment group had a better result. Zhou (2003) applied the method of Liver coursing and Kidney tonifying to treat PMS. Si Ni San and Qi Ju Di Huang Tang were prescribed before menstruation and Liu Wei Di Huang Tang after menstruation.

\section{Spleen Strengthening}

The common herbs for this method are Dang Shen, Fu Ling, Bai Zhu, Ze Xie, Che Qian Zi, Gan Cao, Gu Ya, Mai Ya, and Cang Zhu. Most of these herbs, according to western pharmacology (Chang \& But 1996; Huang 1994), have diuretic, hypoglycemic and liver protective effects (Table 3.5).

The common formulas for this method are Gui Pi Tang, Shen Ling Bai Zhu San, and Fang Ji Huang Qi Tang. 
Li (2000), Zhang and Li (2004) applied Shen Ling Bai Zhu San to treat peri-menstrual diarrhoea in their study with a total effective rate of over $90 \%$. Zhang and Li (2004) recognized that women suffering from peri-menstrual diarrhoea are weak in Spleen and Kidney Yang and the condition will become worse during menstrual period. Shen Ling Bai Zhu San has an action to warm and strengthen the Spleen and the Kidney, and can therefore transform dampness and stop diarrhoea.

Liu (2000) concluded that peri-menstrual swelling has a very close relationship with the Spleen, and that Spleen $Q i$ Deficiency is the major cause of swelling. Liu recognized that Ling Gui Zhu Gan Tang may warm and transform the fluid retained in the body, and prescribed Ling Gui Zhu Gan Tang with additional herbs based on individual patterns to peri-menstrual swelling sufferers. Xu (2003) also applied Ling Gui Zhu Gan Tang with Xian Ling Pi, Ba Ji Tian, Chen $\mathrm{Pi}$ and $\mathrm{Yi} \mathrm{Mu} \mathrm{Cao}$ to 60 women in the treatment group of a study on peri-menstrual swelling. Women in the control group received dihydrocholorothiazide, vitamin B6 and vitamin B1. There was significant difference $(\mathrm{p}<.05)$ between the two groups in the total effective rate, favouring the herbal medicine.

Cao and colleagues (2000) used Gui Pi Tang which is a classic Spleen tonic to treat 20 cases of PMS sufferers with Heart and Spleen deficiency in their study. Whilst Chen (2000) applied Si Ni San with Liu Jun Zi Tang to support the 
Spleen and regulate the Liver for cases of premenstrual diarrhoea caused by the reckless Liver $Q i$ attacking the Spleen.

\section{Blood Moving and Stasis Transforming}

For this treatment method, the herbs that are commonly used are Hong Hua, Tao Ren, Ze Lan, Chuan Shan Jia, and Ji Xue Teng. Most of these herbs, according to western pharmacology (Chang \& But 1996; Huang 1994), have anticoagulant and anti inflammatory effects and can lower blood viscosity (Table 3.6).

Formulas such as Xue Fu Zhu Yu Tang, Shao Fu Zhu Yu Tang, Tao Hong Si Wu Tang, Di Dang Tang, He Tao Cheng Qi Tang, and Dang Gui Shao Yao San are commonly used for this treatment method for blood moving and stasis transforming.

Chen and Chen (2003) monitored the efficacy of the Blood moving and Stasis transforming herbal medicine on premenstrual insomnia in their study. They prescribed the traditional formula Xue Fu Zhu Yu Tang to treat 127 women with this disorder. Chen and colleagues (2003) also applied the Xue Fu Zhu Yu Tang in capsule form to treat 52 cases of PMS women with $Q i$ and Blood stagnation.

Zhao and colleagues (2005) accepted that Liver $Q i$ stagnation and Stomach meridian blockage were the etiology and pathogenesis of PMS. They designed 
a formula with Liver coursing, Stomach strengthening and Blood moving actions to release the stagnation and distension in the Liver and Stomach meridians for 58 women with premenstrual distending pain in their breasts. The formula was modified from Chai Hu Shu Gan Tang and Tao Hong Si Wu Tang. Herbal ingredients include: Chai Hu, Xiang Fu, Chuan Xiong, Zhi Qiao, Chi Shao, Tao Ren, Hong Hua, Qing Pi, Ju Ye, Gua Lou, Gan Cao, Dan Shen, Yu Jin and Shan Yao.

Lin (2000) used Dang Gui Si Ni Tang and Niu Xi as the basis, and added additional herbs to deal with special symptoms to treat 100 cases of peri-menstrual headache. She believes that this formula can warm the Yang $Q i$ and unblock the vessels and meridians, and when the blockage is cleared the pain will be eliminated. Zhang (2004b) recognized that Dang Gui Shao Yao San may regulate the Liver and Spleen, harmonise the Blood and regulate menstruation, thus Dang Gui Shao Yao San with additional herbs was prescribed to 36 PMS sufferers in his study. All the above studies which used Blood moving formulas for the treatment of PMS claimed a success rate over $92 \%$. 
Table 3.2 Pharmacological Effects of Liver Coursing Herbs

\begin{tabular}{|c|c|c|c|c|c|c|c|}
\hline \multirow[b]{3}{*}{ Pharmacological Effect* } & \multicolumn{7}{|c|}{ Liver Coursing Herbs } \\
\hline & Chai $\mathrm{Hu}$ & Dan Pi & Xiang $F u$ & Qing $P i$ & $Y u$ Jin & Dang Gui & Bai Shao \\
\hline & & & & & & & \\
\hline Sedative & $\sqrt{ }$ & $\sqrt{ }$ & $\sqrt{ }$ & & & $\sqrt{ }$ & $\sqrt{ }$ \\
\hline Analgesic & $\sqrt{ }$ & & $\sqrt{ }$ & & & $\sqrt{ }$ & $\sqrt{ }$ \\
\hline Antipyretic & $\sqrt{ }$ & $\sqrt{ }$ & $\sqrt{ }$ & & & & $\sqrt{ }$ \\
\hline Hypothermic & $\sqrt{ }$ & & & & & & \\
\hline Sex hormone like & & & $\sqrt{ }$ & & & & \\
\hline Diuretic & & & & & & $\sqrt{ }$ & \\
\hline Spasmolytic & & $\sqrt{ }$ & $\sqrt{ }$ & $\sqrt{ }$ & & $\sqrt{ }$ & $\sqrt{ }$ \\
\hline Anticoagulant & & & & & $\sqrt{ }$ & $\sqrt{ }$ & \\
\hline Lower blood viscosity & & & & & $\sqrt{ }$ & & \\
\hline Hypoglycemic & & & & & & & \\
\hline Hypotensive & & $\sqrt{ }$ & $\sqrt{ }$ & & & $\sqrt{ }$ & \\
\hline Peripheral vasodilator & & & & & & $\sqrt{ }$ & \\
\hline Coronary vasodilator & & & & & & $\sqrt{ }$ & \\
\hline Antiarrhythmic & & & & & & $\sqrt{ }$ & \\
\hline Liver protective & $\sqrt{ }$ & & & & & $\sqrt{ }$ & $\sqrt{ }$ \\
\hline Anti inflammatory & $\sqrt{ }$ & & $\sqrt{ }$ & & $\sqrt{ }$ & $\sqrt{ }$ & $\sqrt{ }$ \\
\hline Antibacterial & $\sqrt{ }$ & $\sqrt{ }$ & $\sqrt{ }$ & & $\sqrt{ }$ & & $\sqrt{ }$ \\
\hline Antiviral & $\sqrt{ }$ & & & & & & \\
\hline
\end{tabular}

* Chang and But (1996), Huang T (1994) 
Table 3.3 Pharmacological Effects of Liver Clearing and Heat Purging Herbs

\begin{tabular}{|c|c|c|c|c|c|c|c|c|c|c|c|}
\hline \multirow[b]{2}{*}{ Pharmacological Effect* } & \multicolumn{11}{|c|}{ Liver Clearing and Heat Purging Herbs } \\
\hline & $\begin{array}{l}\text { Long } \\
\text { Dan } \\
\text { Cao }\end{array}$ & $\begin{array}{c}D i \\
\text { Huang } \\
\text { (Sheng) }\end{array}$ & $\begin{array}{c}\text { Huang } \\
\text { Qin }\end{array}$ & Zhi Zi & $\begin{array}{c}\text { Chai } \\
\mathrm{Hu}\end{array}$ & Ze Xie & $\begin{array}{l}\text { Dan } \\
\text { Shen, }\end{array}$ & $\begin{array}{l}Y i Y i \\
\text { Ren }\end{array}$ & $\begin{array}{c}\text { Che } \\
\text { Qian } \\
\text { Zi }\end{array}$ & $\begin{array}{c}\text { Хia Ku } \\
\text { Cao }\end{array}$ & $\begin{array}{c}\text { Jи } \\
\text { Ниа }\end{array}$ \\
\hline Sedative & $\sqrt{ }$ & $\sqrt{ }$ & $\sqrt{ }$ & $\sqrt{ }$ & $\sqrt{ }$ & & $\sqrt{ }$ & $\sqrt{ }$ & & & $\sqrt{ }$ \\
\hline Analgesic & & & & $\sqrt{ }$ & $\sqrt{ }$ & & $\sqrt{ }$ & $\sqrt{ }$ & & & \\
\hline Antipyretic & & & $\sqrt{ }$ & & $\sqrt{ }$ & & $\sqrt{ }$ & $\sqrt{ }$ & & & \\
\hline Hypothermic & $\sqrt{ }$ & & & $\sqrt{ }$ & $\sqrt{ }$ & & & $\sqrt{ }$ & & & \\
\hline Sex hormone like & & & & & & & $\sqrt{ }$ & & & & \\
\hline Diuretic & $\sqrt{ }$ & $\sqrt{ }$ & $\sqrt{ }$ & & & $\sqrt{ }$ & & & $\sqrt{ }$ & & \\
\hline Spasmolytic & & & $\sqrt{ }$ & & & $\sqrt{ }$ & & & $\sqrt{ }$ & & \\
\hline Stomachic and digestant & $\sqrt{ }$ & & & & & & & & & & \\
\hline Anticoagulant & & & & & & & $\sqrt{ }$ & & & & \\
\hline Hypoglycemic & & $\sqrt{ }$ & & & & $\sqrt{ }$ & $\sqrt{ }$ & & & & \\
\hline Hypotensive & $\sqrt{ }$ & & $\sqrt{ }$ & $\sqrt{ }$ & & $\sqrt{ }$ & $\sqrt{ }$ & & & $\sqrt{ }$ & \\
\hline Peripheral vasodilator & & & & & & & $\sqrt{ }$ & & & & \\
\hline Coronary vasodilator & & $\sqrt{ }$ & & & & $\sqrt{ }$ & & & & & \\
\hline Liver protective & $\sqrt{ }$ & $\sqrt{ }$ & & $\sqrt{ }$ & $\sqrt{ }$ & $\sqrt{ }$ & & & & & \\
\hline Anti inflammatory & & $\sqrt{ }$ & $\sqrt{ }$ & & $\sqrt{ }$ & & $\sqrt{ }$ & & & & \\
\hline Antibacterial & $\sqrt{ }$ & & $\sqrt{ }$ & $\sqrt{ }$ & $\sqrt{ }$ & & $\sqrt{ }$ & & & $\sqrt{ }$ & \\
\hline Antiviral & & & $\sqrt{ }$ & & $\sqrt{ }$ & & & & & & $\sqrt{ }$ \\
\hline
\end{tabular}

* Chang and But (1996), Huang T (1994) 
Table 3.4 Pharmacological Effects of Liver and Kidney Nourishing Herbs

\section{Liver and Kidney Nourishing Herbs}

\begin{tabular}{|c|c|c|c|c|c|c|c|c|}
\hline Pharmacological Effect* & $\begin{array}{c}\text { Di Huang } \\
\text { (Shu) }\end{array}$ & Shan Yao & $\begin{array}{c}\text { Shan Zhu } \\
Y u\end{array}$ & $\begin{array}{c}\text { Gou } Q i \\
Z i\end{array}$ & $\begin{array}{l}\text { Shan } \\
\text { Shen }\end{array}$ & $\begin{array}{c}\text { Mai } \\
\text { Dong }\end{array}$ & $\begin{array}{c}N u \text { Zhen } \\
Z i\end{array}$ & $\begin{array}{c}\text { Yin Yang } \\
\text { Нио }\end{array}$ \\
\hline Sedative & $\sqrt{ }$ & & & & & $\sqrt{ }$ & & \\
\hline Analgesic & & & & & $\sqrt{ }$ & & & \\
\hline Antipyretic & & & & & $\sqrt{ }$ & & & \\
\hline Sex hormone like & & & & & & & & $\sqrt{ }$ \\
\hline Diuretic & $\sqrt{ }$ & & $\sqrt{ }$ & & & & & \\
\hline Hypoglycemic & $\sqrt{ }$ & $\sqrt{ }$ & $\sqrt{ }$ & $\sqrt{ }$ & & & & $\sqrt{ }$ \\
\hline Hypotensive & & & $\sqrt{ }$ & & & & & $\sqrt{ }$ \\
\hline Coronary vasodilator & $\sqrt{ }$ & & & & & & $\sqrt{ }$ & $\sqrt{ }$ \\
\hline Antiarrhythmic & & & & & & & & $\sqrt{ }$ \\
\hline Liver protective & $\sqrt{ }$ & & & $\sqrt{ }$ & & & & \\
\hline Anti inflammatory & $\sqrt{ }$ & & $\sqrt{ }$ & & & & & \\
\hline Antibacterial & & & $\sqrt{ }$ & & & $\sqrt{ }$ & $\sqrt{ }$ & $\sqrt{ }$ \\
\hline Antiviral & & & & & & & & $\sqrt{ }$ \\
\hline
\end{tabular}

* Chang and But (1996), Huang T (1994) 
Table 3.5 Pharmacological Effects of Spleen Strengthening Herbs

\begin{tabular}{|c|c|c|c|c|c|c|c|c|c|}
\hline \multirow[b]{3}{*}{ Pharmacological Effect* } & \multicolumn{9}{|c|}{ Spleen Strengthening Herbs } \\
\hline & Dang & Fu Ling & Bai Zhu & Che & Gan & Gu Ya & Mai Ya & Cang & Ze Xie \\
\hline & Shen & & & Qian Zi & $\mathrm{Cao}$ & & & Zhu & \\
\hline Sedative & & $\sqrt{ }$ & & & & & & & \\
\hline Analgesic & & & & & $\sqrt{ }$ & & & & \\
\hline Antipyretic & & & & & $\sqrt{ }$ & & & & \\
\hline \multicolumn{10}{|l|}{ Sex hormone like } \\
\hline Diuretic & & $\sqrt{ }$ & $\sqrt{ }$ & $\sqrt{ }$ & & & & $\sqrt{ }$ & $\sqrt{ }$ \\
\hline Spasmolytic & & & & & $\sqrt{ }$ & & & & $\sqrt{ }$ \\
\hline Stomachic and digestant & & & & & & $\sqrt{ }$ & $\sqrt{ }$ & & \\
\hline Hypoglycemic & & & $\sqrt{ }$ & & & & $\sqrt{ }$ & $\sqrt{ }$ & $\sqrt{ }$ \\
\hline Hypotensive & & & $\sqrt{ }$ & & & & & & $\sqrt{ }$ \\
\hline Peripheral vasodilator & & & $\sqrt{ }$ & & & & & & \\
\hline Coronary vasodilator & $\sqrt{ }$ & & & & & & & & $\sqrt{ }$ \\
\hline Liver protective & & $\sqrt{ }$ & $\sqrt{ }$ & & $\sqrt{ }$ & & & & $\sqrt{ }$ \\
\hline Anti inflammatory & & & & & $\sqrt{ }$ & & & & \\
\hline Antibacterial & & & & & $\sqrt{ }$ & & & $\sqrt{ }$ & \\
\hline
\end{tabular}

* Chang and But (1996), Huang T (1994) 
Table 3.6 Pharmacological Effects of Blood Moving and Stasis Transforming Herbs

\begin{tabular}{|c|c|c|c|c|c|}
\hline \multirow[b]{2}{*}{ Pharmacological Effect* } & \multicolumn{5}{|c|}{ Blood Moving and Stasis Transforming Herbs } \\
\hline & Hong Hua & Tao Ren & Ze Lan & Chuan Shan Jia & Ji Xue Teng \\
\hline Sedative & $\sqrt{ }$ & & & & \\
\hline Analgesic & $\sqrt{ }$ & & & & \\
\hline Anticoagulant & $\sqrt{ }$ & $\sqrt{ }$ & & & \\
\hline Lower blood viscosity & & & $\sqrt{ }$ & $\sqrt{ }$ & \\
\hline Hypoglycemic & $\sqrt{ }$ & & & & \\
\hline Hypotensive & & & & & $\sqrt{ }$ \\
\hline Peripheral vasodilator & & & $\sqrt{ }$ & & \\
\hline Anti inflammatory & $\sqrt{ }$ & $\sqrt{ }$ & & $\sqrt{ }$ & \\
\hline
\end{tabular}

* Chang and But (1996), Huang T (1994) 


\subsubsection{Outcome of Herbal Treatment Trials in PMS}

All these PMS clinical studies reported a positive and outstanding result. Gao and Chen (1994) summarised the results of 30 studies of PMS using Chinese herbal medicine in China. The summary shows 651 cases (75.6\%) with full recovery, 177 cases $(20.6 \%)$ with some effect and $33(3.8 \%)$ cases with no effect out of a total of 861 cases of PMS.

The author reviewed 124 clinical studies using Chinese herbs for the treatment of PMS from 1996 to 2005 (see Chapter 5). The summary of the 2179 cases in the treatment groups of these trials shows $62.1 \%$ with full recovery, $8.1 \%$ with outstanding outcome, $24.9 \%$ with some improvement and $4.9 \%$ with no effect. The total effective rate was reported in 21 controlled trials. The average total effective rates of the treatment and control groups were $92.2 \%$ and $64.2 \%$ respectively. The analysis also revealed that the change effect of the active treatment was larger than the control treatment in the 'full recovery' and 'outstanding outcome' categories.

\subsubsection{Other Therapies}

Besides the treatment strategies described above which involve the application of Chinese herbal medicine internally, other methods which include the application of acupuncture, auricular point pressing, massage and external applications are also documented. Some reports claim that not only are external 
treatment strategies just as effective as Chinese and western internal medication, they are much more economical and simple in operation.

\section{$\underline{\text { Acupuncture }}$}

Sun and Guo (2004) compared the clinical therapeutic effects of acupuncture and western medicine on PMS. Sixty-one cases were divided into two groups. The acupuncture group was treated with acupuncture at main points Baihui (Ren 20), Guanyuan (Ren 4), Danzhong (Ren 17), Neiguan (P. 6), Taichong (Liv.3), Sanyinjiao (Sp. 6) and Back-shu points as adjuvant points, and the medicine control group was treated with Provera (medroxyprogestorone acetate). After three months of treatment, there was a significant difference ( $\mathrm{P}$ $<.05)$ between the two groups in the total symptom scores indicating that acupuncture therapy is superior to the Provera treatment in relieving PMS symptoms.

Zhang and Chen (2000) applied acupuncture and Chinese herbal medicine to treat PMS. Fifty PMS women received acupuncture [main Points: Zhongji (Ren 3), Guanyuan (Ren 4), Zusanli (St. 36), Sanyinjiao(Sp. 6)] and Chinese herbal medicine (modified Xiao Yao San) three to four days before menstruation, and another forty PMS women in the study took oryzanol, vitamin B6 and Vitamin A. The acupuncture and herbal medicine group had a total effective rate of $96 \%$ while the control group had a total effective rate of only $82.5 \%$. An and colleagues (2005) used the same main points plus psychotherapy to treat 42 PMS sufferers. The women $(n=42)$ in the control group also received oryzanol, vitamin B6, Vitamin A and Xiao Yao Wan. In both studies, the results indicated 
that the acupuncture groups achieved better total effective rates than the control groups.

$\mathrm{Yu}$ and colleagues carried out an evaluation of the clinical therapeutic effects and the safety aspect of acupuncture treatment for premenstrual syndrome in 2005. Eight controlled studies involving 807 women were analysed. They concluded that acupuncture can be effective in treating PMS. However, they had doubts in the standard and quality of these studies and indeed all the studies conducted up to this date. It is considered that more randomized controlled trials are necessary to assess the actual effectiveness and to identify any adverse effect of acupuncture for the treatment of PMS.

\section{$\underline{\text { Ear Reflex Points }}$}

According to the Holographic information, each area of the ear lobe corresponds to a different anatomical part of the body. Stimulation of the ear reflex points exerts certain therapeutic effects on those parts of the body with which they are associated.

Jiang and colleagues performed a clinical study on treatment of PMS by auricular point pressing in 2002. Sixty-three participants were divided into three groups i.e. the auricular point pressing group, Chinese patent medicine group and western medicine group. The ear reflex points: Liver, Kidney Heart, Spleen, Endocrine, Uterus, Ovary, Sympathetic and Subcortex were used as main points, with adjuvant points Occiput, Forehead and Thoracic vertebrae for participants with Liver stagnation; Sanjiao and Cymba-conchae for participants 
with Spleen deficiency; Shenmen and Pituitary for participants with Blood deficiency. Patent herbal medicine Xiao Yao San was prescribed to participants with Liver stagnation, Shen Ling Bai Zhu San was prescribed to participants with Spleen deficiency, and Dang Gui Bu Xue Wan was prescribed to participants with Blood deficiency in the Chinese patent medicine group. Oryzanol was prescribed to participants of the western medicine group. Significant differences $(\mathrm{p}<.05)$ were observed in the total effective rates among the three groups. The total effective rates were $100 \%, 73.33 \%$ and $68.75 \%$ in the auricular point pressing group, the Chinese patent medicine group and the western medicine group respectively. They concluded that auricular point pressing is safe and effective for the treatment of PMS.

Zheng and colleagues (2002) applied auricular point pressing (ear points: Liver, Kidney, Uterus, Endocrine, Ovary and Shenmen) and herbal medicine (modified Xiao Yao San) together to treat PMS in 100 cases. He and Li (2000) applied auricular point pressing (ear points: Shenmen, Uterus, Endocrine, Subcortex, Liver, Heart and Kidney) and herbal medicine (modified Gan Mai Da Zao Tang) to treat peri-menstrual emotional disturbances. Zhang (2002) used auricular point pressing together with acupuncture to treat peri-menstrual breast pain and distension. All these studies reported good results.

\section{$\underline{\text { Massage }}$}

Zhang (2003d) recognized that PMS has a close relationship with the Ren Meridians and the Urinary Bladder Meridians. The Ren Meridians govern Yin and the reproductive system of the body. The Urinary Bladder Meridians 
contains Back-Shu points of organs. A study based on the application of massage along these two meridians on one hundred and eight PMS women before menstruation was carried out with good result. Wang and colleagues (2003) applied massage followed by acupuncture before menstruation to treat PMS.

\title{
$\underline{\text { External Applications }}$
}

Jiang and colleagues (1995) used external herbal packs to treat PMS. Raw herbs including Chai Hu, Xiang Fu, Xuan Hu, Dang Gui, Chuan Xiong, Ai Ye, and She Chuang $Z i$ were packed in a cotton bag and applied to the women's umbilical region 3-5 days before and during menstruation for the treatment group, whilst bran skin was packed in the bag for the control group.

\subsection{Summary}

\begin{abstract}
Although TCM only identified and discussed the premenstrual or peri-menstrual symptoms in the classic literatures, the descriptions of the symptoms mirror the western biomedical phenomena of PMS very closely.
\end{abstract}

While the exact cause and pathophysiology of PMS is not completely understood in western science, TCM theorizes that Liver $Q i$ stagnation together with Yin-blood deficiency in female are the root causes of pathological changes in PMS. The cyclic occurrence of the symptoms is explained as being related to the changes of the excess and deficiency of the blood and $Q i$, and the fullness 
and emptiness of the Chong and Ren meridians during this phase. The variety of symptoms and symptom severity are related to the state of $Q i$, Blood, Yang, Yin, and individual organs of the woman sufferers.

Traditionally, TCM practitioners diagnose peri-menstrual disorders into patterns of disharmony within each major symptom disorder. However, contemporary Chinese PMS researchers follow diagnostic criteria from different sources, and this may affect the reliability and the result of treatment efficacy in applying Chinese medicine for PMS.

TCM concept emphasizes the unity within the human body, and as such the functions of all the internal organs are interconnected and interdependent. TCM practitioners are able to apply pattern identification and treatment in many ways based on their own experiences and personal preferences. Classification of TCM patterns in premenstrual/peri-menstrual disorders can be based on the general manifestation of PMS, specific symptoms of peri-menstrual disorders, or the primary causes of PMS. While western medicine applies acute hormones, Selective serotonin reuptake inhibitors (SSRIs) and symptomatic treatment for the disorder (Freeman and Halbreich 1998). TCM treatment strategies include treatment for the whole syndrome, the primary symptoms and the organ systems and also treatment according to menstrual cycle phases. Treatment therapy varies from Chinese herbal medicine, acupuncture, auricular point pressing, massage, to external application. 
It is clear that PMS is a condition experienced by numerous women worldwide for which Chinese medicine may be a most suitable addition as an effective alternative treatment to western medicine. However, the conflict between traditional and modern methods of diagnosis in PMS resulting in too many classifications and treatment strategies may confuse practitioners. Also, the confidence in this treatment is largely based on clinical experience that accords unquestioning respect to traditional practices. Although some research has been carried out, more rigorous clinical trials on the use of Chinese herbs to treat PMS are necessary to satisfy western demands for hard, scientifically and thoroughly researched evidence. 
Page

\section{Chapter 4: Methodological Issues for Research on Premenstrual Syndrome}

4.1 Research Design

4.4.1 Outcome Measures according to Statistical Significance 134

4.4.2 Outcome Measures according to Clinical Significance 135

4.4.3 Retrospective and Prospective Measures 137

$\begin{array}{lll}\text { 4.4.4 Instruments } & 138\end{array}$

4.4.4.1 Instruments for Menstrual Symptoms $\quad 139$

4.4.4.2 Instruments for Psychological Factors 140

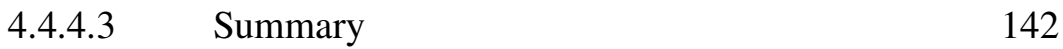




\subsection{Research Design}

Research design is used to structure the research project, to bring together all major parts of the project namely, samples, methods of assignment, measures and treatment work, and to aid in the solution of the research question, and also to maintain control (Trochim, 2000).

Experimental designs are regarded as the most rigorous of all research designs. They are the strongest design with respect to internal validity/causal assessment. Experimental designs are characterized by the randomization of subjects into treatment and control groups. The experimental group receives the program/treatment and the control group/s receives no, placebo, conventional or other program/treatment. The same measures and observations are applied to all groups and statistical comparisons are made.

One of the most common designs utilizing two groups of clients in clinical trials is the crossover design (Spilker, 1996). In this design, each client receives both treatments being compared in the trial. In effect, each subject is their own control. The treatment outcomes are compared using repeated measurements taken on each subject at pre-determined times. If one of the treatments is a placebo, then the effects of the trial medicine or substance may be expressed as the difference between subjects' responses to the two treatments. For the same sample size, the crossover design is more sensitive in detecting differences between two treatment groups than a parallel groups trial. The use of a 
cross-over design in a study assures that everyone would eventually get the treatment to avoid compensatory rivalry and/or resentful demoralization. However, the statistical power from a crossover design study is adversely affected by attrition of participants and missing data when participants withdraw due to disappointment or disillusion with withheld treatment or other control conditions. To overcome expected attrition it is usual to recruit more participants than the minium required to provide adequate power to detect a significant difference at the $5 \%$ level.

\subsubsection{Stratified Allocation}

In Chinese medicine, each disorder is understood according to different TCM (traditional Chinese medicine) patterns of disharmony. In a Randomized Block Design, the samples are divided into homogeneous blocks/subgroups, and the experimental design of the program is implemented within each block. The variability within each block is less than the variability of the entire sample, thus each estimate of the treatment effect within a block is more efficient than the estimates across the entire sample (Trochim, 2000). The advantage of using this sampling design in a TCM research is that both the treatment effects within subgroups (TCM patterns of the disorder) and the overall treatment effects (the disorder) between groups can be estimated. 


\subsection{Diagnostic Process of Premenstrual Syndrome}

There is no hormonal or other laboratory test available to confirm a diagnosis of premenstrual syndrome (PMS), and no singular consensus for a diagnosis of clinically significant PMS (Halbreich 2004).

The essential components of a PMS diagnosis include confirming

- $\quad$ that the symptoms occur premenstrually and are recurrent with menses;

- that the symptoms impair usual functioning to a self reported mild, moderate or severe level;

- that the symptoms are not due to another physical or mental disorder.

The first step in the diagnostic process is the prospective confirmation of cyclicity and severity. Daily symptom reports may serve two functions, (i) confirmation of the patient's overall subjective statement, and (ii) confirmation of the patient's report of cyclicity of symptoms and their synchronicity with the menstrual cycle. Daily symptom reports on pre-developed forms maintained by the patient for a minimum of two cycles are required to confirm the reported PMS symptoms and their cyclical nature. When a client responds to recruitment invitations for a PMS treatment trial, daily rating forms should be provided, with verbal and written instructions on completion requirements. The charted forms should be brought to the initial visit, so that the symptoms can be reviewed at the first visit.

The symptoms should be assessed carefully in both the postmenstrual and premenstrual phases of the cycle. The postmenstrual symptoms can range from 
asymptomatic levels to subclinical levels in women with PMS. The degree of change in symptom severities between postmenstrual and premenstrual symptoms needs to be clearly defined. Some researchers compare symptom severities experienced over a variable number of premenstrual days (usually from -7 to -4 days prior to menstruation) to those experienced over a variable number of postmenstrual days (typically from 1 to 7 days). Others compared symptoms over a number of premenstrual days to symptoms experienced over the full cycle (Budeiri et al. 1994). The most commonly accepted practice used in PMS studies for confirmation is that symptom ratings must increase by $30 \%$ or more during the week prior to menstruation compared with the first week following cessation of menses in at least two consecutive cycles (Gallant et al. 1992).

Functional impairment is another required criterion for the diagnosis of PMS. This information should include the domains (physical, emotional, behavioural) affected, the severity of the impairment and its occurrence in relation to the menstrual cycle (Freeman 2003). The woman's perception of her impairment due to PMS can be obtained by clinical inquiry during the initial visit.

Differential diagnosis and ruling out other conditions can also be made in the initial visit by careful inquiry into the physical, mental, obstetric and gynecologic histories of the client. If possible, blood and urine tests should be carried out to rule out general systemic competing diagnoses such as diabetes, thyroid or adrenal disorders. 


\subsection{Selection Criteria}

Diagnostic criteria are important for distinguishing between PMS and other disorders, determining the prevalence of the disorder, and most importantly, for determining effective treatment (Freeman 2003). Since there are no universally accepted diagnosis guidelines/standards for quantifying the three important components of PMS diagnostics, researchers must provide this information in order to understand the symptom status of study groups.

Diagnostic criteria must specify the broad range of symptoms, the temporal and cyclic pattern of the symptoms and the degree of symptom severity, which differentiates clinically significant PMS from normal menstrual cycle changes and other physical or mental disorders. Halbreich $(1997 ; 2004)$ proposed the main diagnostic inclusion and exclusion criteria for PMS as follows:-

The inclusion criteria include:

- Any mood, behavior or physical symptom(s) or cluster(s) of symptoms that occur recurrently and cyclically in the luteal phase of the menstrual cycle;

- The symptom(s) subside with menses and remain in remission for at least one week of the follicular phase in most menstrual cycles

- The symptom(s) cause emotional or physical distress, suffering or impairment in daily functioning;

- The recurrence, cyclicity, and timing in the cycle, the severity of symptoms, and the symptom-free period are documented by daily symptom monitoring and/or reports. 
The exclusion criteria include:

- Not menstruating;

- Any chronic major mental disorder;

- Physical disorders severe enough to warrant treatment;

- Past history or family history of vulnerability to possible adverse effects of the medication studied;

- Alcoholism or substance abuse within 6-12 months prior to the trial;

- Use of oral contraceptives or other hormonal interventions within 3 months prior to screening;

- Use of most medications within a time frame of five half-life periods of each medication;

- Laboratory values of required testes outside the well-defined normal range.

\subsection{Outcome measures}

Measures of severity of specific symptom clusters are important for determining which symptoms respond to treatment. PMS is characterized by both psychological and physical symptoms. PMS studies of Australia and China (Gotts et al. 1995; Zhao et al. 1998; Walsh \& Polus 1999) revealed that affective symptoms irritability/anger, depression and anxiety were the most frequently reported symptoms. Additional standardized measures of mood dimensions such as irritability, depression and anxiety, impairment of functioning and quality of 
life are important supplements to the menstrual symptom reports and make it possible to compare symptoms and severity with other study groups.

\subsubsection{Outcome Measures according to Statistical Significance}

Currently, proof of efficacy of an active treatment component in most research of PMS is considered as demonstration of a statistically significant better-than-placebo improvement in key rating measures. The treatment results between the pretest, the posttest, and the follow-up of both experimental and placebo treatment are measured and compared on the degree of changes in the occurrence and severity of the premenstrual somatic, mood and behavioral symptoms. Some researchers measure the success of treatment based on a percentage decrease at post-treatment in total scores. Halbreich (2004) recommends that the primary outcome measure in clinical trials should be based on 'not meeting entry criteria post-treatment'. The widely cited criterion of $30 \%$ marked change between postmenstrual (follicular phase) and premenstrual (late luteal phase) symptoms levels was suggested by the NIMH in 1983 (Hamilton et al., 1984). However, this criterion is intended to relate to the scale used, and a definition of the minimal late luteal total score on symptom severity is needed to meet the criteria for PMS. 


\subsubsection{Outcome Measures according to Clinical Significance}

Statistical significance relates to the question of whether or not the results of a statistical test meet an accepted criterion level, while clinical significance relates to whether the treatment has a successful outcome and/or the clients feel improved or cured. Kendall and Grove (1988) define clinical significance as when "once troubled and disordered clients are now, after treatment, not distinguishable from a meaningful and representative non-disturbed reference group”. Clinical significance can be demonstrated through the calculation on the reduction of scores, percent improvement and /or the effect size.

\section{Calculation of the reduction of scores}

Becker (1997) points out that if the mean of the treated group falls within \pm 1 SD (Standard Deviation ) of the mean of the normative group then the treated group is undistinguishable from the normative group, and an individual who falls to or below this cut-off score is viewed as having successful outcome or can be perceived as "cured". At the same time, if the mean of a group is beyond $+1 \mathrm{SD}$ of the mean of the normative group then this group is disturbed (troubled and disordered).

A change in clinical significance can be demonstrated when scores of a group of clients who are troubled and disordered prior to the treatment and the mean of their post treatment scores falls at or to within 1 SD from the mean of that group. In other words, a "cure" can be claimed to have occurred when the post 
treatment scores of those clients have moved from beyond +1 SD of the normative group's mean to within $\pm 1 \mathrm{SD}$.

\section{Calculation of Percent Improvement}

Another way to determine the significance of clinical change is the calculation of the percent improvement between baseline and after treatment or between experimental group and control group where a $25 \%$ improvement or greater represents, by convention, a clinical significant difference (Long, 1995). Percent improvement between pretest and posttest is estimated by the ratio of the mean difference between the two groups divided by the mean of the pretest group. The percent improvement between experimental and control groups is estimated by the difference between the percent improvement of the experimental and control groups.

\section{Calculation of Effect Size}

The standardized difference in clinical treatment effect between two groups is commonly determined by the calculation of the effect size (ES). Effect size which was proposed by Cohen (1988) is estimated by the ratio of the mean difference between two groups divided by the standard deviation of either group. Although Cohen (1988) argued that the standard deviation of either group can be used when the variances of the two groups are homogeneous, in practice the pooled standard deviation is commonly used (Rosnow \& Rosenthal, 1996). The pooled standard deviation is the square root of the average of the squared standard deviation. The sizes of Effect were defined as "small, $d=.2$ ", “medium, $d=.5 "$ and large, $d=.8$ " by Cohen (1988). 


\subsubsection{Retrospective and Prospective Measures}

Both retrospective and prospective measures have their own advantages and disadvantages. Prospective reports usually demonstrate a more accurate estimation of symptom cyclical occurrence and severity which helps to confirm the diagnose and provides an easier categorization of patients with PMS (Budeiri et al. 1994). Prospective reports are the most popular and acceptable method used today as they have less reliance on memory. The current diagnostic standard requires confirmation of subjective symptom reports by prospective daily diaries. However, daily rating may result in biased samples through nonadherence and is rather demanding for participants leading to high drop-out rates in studies (Haywood et al. 2002). Richter and colleagues (1984) reported that most of their subjects found the daily record keeping unacceptable and withdrew from the trial; Gise and colleagues (1990) reported that $63 \%$ of their participants failed to comply with a two month daily prospective record keeping. Miller and colleagues (1999) reported that only 2 out of the 16 participants completed the observation period of their study. Sveindottir and Backstrom (2000) reported that 15 of the 98 participants in their study only charted the menstrual diary for no more than 15 days and cited the charting as too much of a problem. Gise and colleagues (1990) pointed out that this bias is more likely if the charting is longer than 2 months, which is the minimum rating time for a diagnosis of PMS. Retrospective symptom reports reflect average experiences and can distinguish between premenstrual and postmenstrual symptoms (Hart et al. 1987), but they have been criticised for 
providing over-estimations of symptom severity (Endicott and Halbreich 1982) biased by cultural expectations and heavily reliant upon participants' memories of past menstrual related symptoms (Richardson 1990; Woods et al. 1982a). On the other hand, Hart and colleagues (1987) suggested that retrospective reporting is relatively valid for interpreting the severity of premenstrual symptoms, Kim (2004) and Steiner and colleagues (2005) pointed out that symptoms on the prospective and retrospective reports were found to be similar or no significant difference in their studies. There is value in retrospective reports, other than being less demanding for participants, for consistency with other well controlled trials already reported in the public domain.

\subsubsection{Instruments}

The adequacy of the method for measurement is a crucial aspect of PMS research. Menstrual symptoms have been measured by a variety of instruments including both retrospective and prospective symptom reports. A number of instruments have been used for rating PMS symptoms (Moos, 1968; Abraham, 1980; Mortola et al., 1990; Thys-Jacobs et al., 1995; Freeman et al., 1996). There is no data-based evidence of superiority of one type of rating scale over another in determining a diagnosis of PMS, although there is considerable variability in their scope (Freeman 2003). 


\subsubsection{Instruments for Menstrual Symptoms}

\section{Moo's (1985) Menstrual Distress Questionnaire:}

The Menstrual distress Questionnaire (Moos, 1968, 1969) is the most widely used record to measure menstrual cycle symptoms. It consists of 47 items arranged into eight sub-scales namely Pain, Water Retention, Autonomic Reaction, Negative Affect, Impaired Concentration, Behavioural Change, Arousal and Control (see Table 2.2). There are two forms of the revised version of Menstrual Distress questionnaire (1985). Form C enables a woman to report her menstrual cycle experiences in three phases of her last menstrual cycle whereas Form $\mathrm{T}$ enables a women to describe her reactions or symptoms for one or more specific days. Form $\mathrm{T}$ is suitable for repeated assessment of women's reactions over time.

The symptoms in Form $\mathrm{T}$ are rated on a 5-point Likert scale, ranging from (0) no reaction; (1) present mild; (2) present moderate; (3) present strong to (4) present severe. The internal consistencies of the eight clusters sub-scales of Form $\mathrm{T}$ are moderate to high, varying from $\alpha=.94$ (Negative affect) to $\alpha=.63$ (Control) (Moos, 1985). The test-retest reliability of the Menstrual Distress questionnaire is moderately good with $r=0.88$ (Markum 1976).

\section{Abraham's (1980) Menstrual Symptom Diary:}

The menstrual symptom diary is a prospective menstrual symptom diary modified from Moo's menstrual distress questionnaire by Abraham (1980a,b) that showed the greatest changes premenstrually. It includes only 19 symptoms 
(Nervous tension; Mood swings; Irritability; Anxiety; Weight gain; Swelling of extremities; Breast tenderness; Abdominal bloating; Headache; Craving for sweet; Increased appetite; Heart pounding; Fatigue; Dizziness or faintness; Depression; Forgetfulness; Crying; Confusion and Insomnia).

The 19 PMS symptoms are grouped into four cluster categories of PMT-A (first four symptoms), PMT-H ( $5^{\mathrm{TH}}$ to $8^{\mathrm{TH}}$ Symptoms), PMT-C ( $9^{\text {th }}$ to $14^{\text {th }}$ symptoms) and PMT-D (last five symptoms) in the original menstrual symptom diary of Abraham (see Table 2.3). The symptoms are graded on a 4 point Likert scale where $0=$ non existent, $1=$ mild present but does not interfere with activities, 2 $=$ moderate present and interferes with activities but not disabling, and $3=$ severely disabling, unable to function. Internal consistency of the diary based upon Cronbach's coefficient alpha $(\alpha)$ is 0.82 (Chau \& Chang 1999).

\subsubsection{Instruments for Psychological Factors}

\section{Short Form Beck Depression Inventory:}

The short form Beck depression inventory is a self-reported screening instrument introduced by Beck and Beck (1972) to detect and measure the presence of depressed cognitive, emotional and psychological symptoms in clinical practice. It consists of 13 symptoms or attitudes including Sadness, Pessimism, Sense of failure, Dissatisfaction, Guilt, Self-dislike, Self-harm, Social withdrawal, Indecisiveness, Self-image change, Work difficulty, Fatigability, and Anorexia. Each of these items is rated on a 4-point Likert 
scale ranging from zero to three in terms of severity where $0=$ None/Not at All and $3=$ Severe. The total score of the BDI was obtained by simple adding the scores of all items. The estimated degree of depression according to the scores obtained from the short form BDI ranges from: none or minimal depression (0-4); mild depression (5-7); moderate depression (8-15); to severe depression (16 and over). This form requires about five minutes to complete. The internal consistency (coefficient alpha) of the short form BDI is $\alpha=.81$ (Knight, 1984) and $\alpha=.83$ (Reynolds \& Gould, 1981).

\section{Spielberger's (1979) State-Trait Personality Inventory}

The state-trait personality inventory consists of six subscales: state and trait anxiety, state and trait curiosity, and state and trait anger. The state items of the inventory examine the feeling at a particular moment in time while the trait items test the general feelings. The state-anxiety and state-anger subscales have been widely used for measuring the negative characteristics during premenstrual period.

Both state-anxiety and state-anger inventories consist of a 10-item and a 4-point Likert scale. Where $1=$ not at all, 2 = somewhat; 3 = moderately so; and $4=$ very much so. The range of possible scores for each subscale can vary from a minimum of 10 to a maximum of 40 . Higher scores indicate higher levels of the emotion. The internal consistency is satisfactory with $\alpha=.88$ for the anger subscale, and $\alpha=.94$ for the anxiety subscale (Spielberger 1979). 


\section{Perceived Stress Scale (Cohen, Kamarck \& Mermelstein 1983):}

The Perceived Stress Scale (PSS) measures the degree to which situations in one's life are appraised as stressful. (Remor \& Carrobles, 2001). The PSS has been widely used as a psychological instrument for measuring the role of non-specific appraised stress in the aetiology of disease and behavioural disorders and as an outcome measure of experienced levels of stress. It is a 14-item self report 5 -point Likert type scale $(0=$ never; $1=$ almost never; $2=$ sometimes; 3 = fairly often; and 4 = very often). The PSS scores are obtained by reversing the scores on the seven positive items, and then summing across all 14 items. A high summary score indicates high psychological distress. Coefficient alpha ranges between $0.84-0.86$, test-retest correlation is 0.85 (after 2 days) and 0.55 (after six weeks) (Cohen, Kamarck \& Mermelstein 1983).

\subsubsection{Summary}

The Moo's Menstrual Distress Questionnaire is the most widely used measure for menstrual cycle symptoms. Form $\mathrm{T}$ can be used for repeated assessment of the women's symptoms occurrence and severity during premenstrual phase over time, and for consistency with other trials in comparison of the results. Abraham's Menstrual Symptom Diary can be used as a prospective record keeping to meet the requirement of current diagnostic standard (The American College of Obstetricians and Gynecologists and National Institute of Mental Health), for accurate estimation of individual symptoms, and for comparison of symptoms between the nutritional aetiological patterns with TCM patterns. 
Additional psychological instruments which are widely used such as the short

form Beck Depression Inventory, the Spielberger's state-anxiety and state-anger inventories and the Perceived Stress Scale may further assess the negative characteristics of the PMS sufferers during premenstrual period. 
Chapter 5: A Review of Clinical Research of Premenstrual Syndrome

Page Treatment Using Herbal Medicine in Chinese Medical Journals 1996-2005

5.1 The Objective of Review

$5.2 \quad$ Study Selection

5.3 Data Sources

$\begin{array}{llr}5.4 & \text { Results } & 148\end{array}$

$\begin{array}{lll}\text { 5.4.1 General Description } & 148\end{array}$

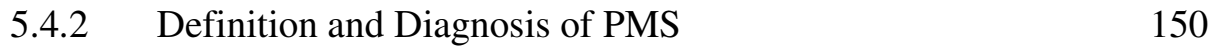

$\begin{array}{ll}5.4 .3 & 150\end{array}$

5.4.4 Measure of Studies 153

5.4.5 Outcome of Studies 154

$\begin{array}{lll}\text { 5.4.6 Treatment Methods } & 161\end{array}$

$\begin{array}{lll}\text { 5.5 Discussion and Future Directions } & 163\end{array}$ 


\subsection{The Objective of Review}

The history of herbal medicine being the primary medicine for the treatment of diseases in China spans over thousands of years. Specific formulas for various diseases have been developed mainly through experience, or more accurately, by trial and error. Many trials have been carried out to verify the effectiveness of Chinese herbal medicine for the treatment of premenstrual syndrome (PMS)

in China in recent years. However, these trials have an image to be defective or lacking in scientific basis especially in the eyes of professionals in the western world. The author is therefore conducting a review of the clinical studies on PMS using traditional Chinese medicine (TCM) in Chinese medical journals to clarify the present standard and need of randomised and controlled experimental trial for Chinese herbal medicine for PMS.

\subsection{Study Selection}

Studies are eligible for analysis if they:-

- are directly relevant to medical management of PMS

- are clinical trials

- report the result of the study in detail

- use Chinese herbal medicine as treatment media

- are not combined with any other treatment method

- are published in Chinese TCM journals 
- are not reviews, tutorials, discussion papers or conference proceedings

\subsection{Data Sources}

The author carried out an electronic search for articles on PMS from 1996 to 2005 in Chinese TCM journals through two electronic data bases i.e. CNKI net (www.cnki.net) and Wanfang data (www.wanfangdata.com.cn), and the Chinese Bio-Medicine Disc (Institute-of-Medical-Information, 2000). The terms 'PMS' 'PMT' 'Peri-menstrual' 'Premenstrual' combined with 'Chinese medicine' were used as key words for the search. However, search for clinical trials using Chinese medicine had to be carried out by hand searching. If the nature of the articles was unclear from the title and abstract, the full article was reviewed. Altogether, 184 trials were identified out of 1362 records of TCM articles on PMS, peri-menstrual disorders, and/or premenstrual disorders. Further screening resulted on the elimination of 26 trials using other treatment media e.g. acupuncture, ear points pressing, massage, 22 trials using combination of Chinese and western medicine, one trial used herbs in a compress applied externally and 11 articles reporting studies that had already been published. Ultimately, a total of 124 clinical trials met the eligibility criteria of this review and the search result is shown in Figure 5.1. 


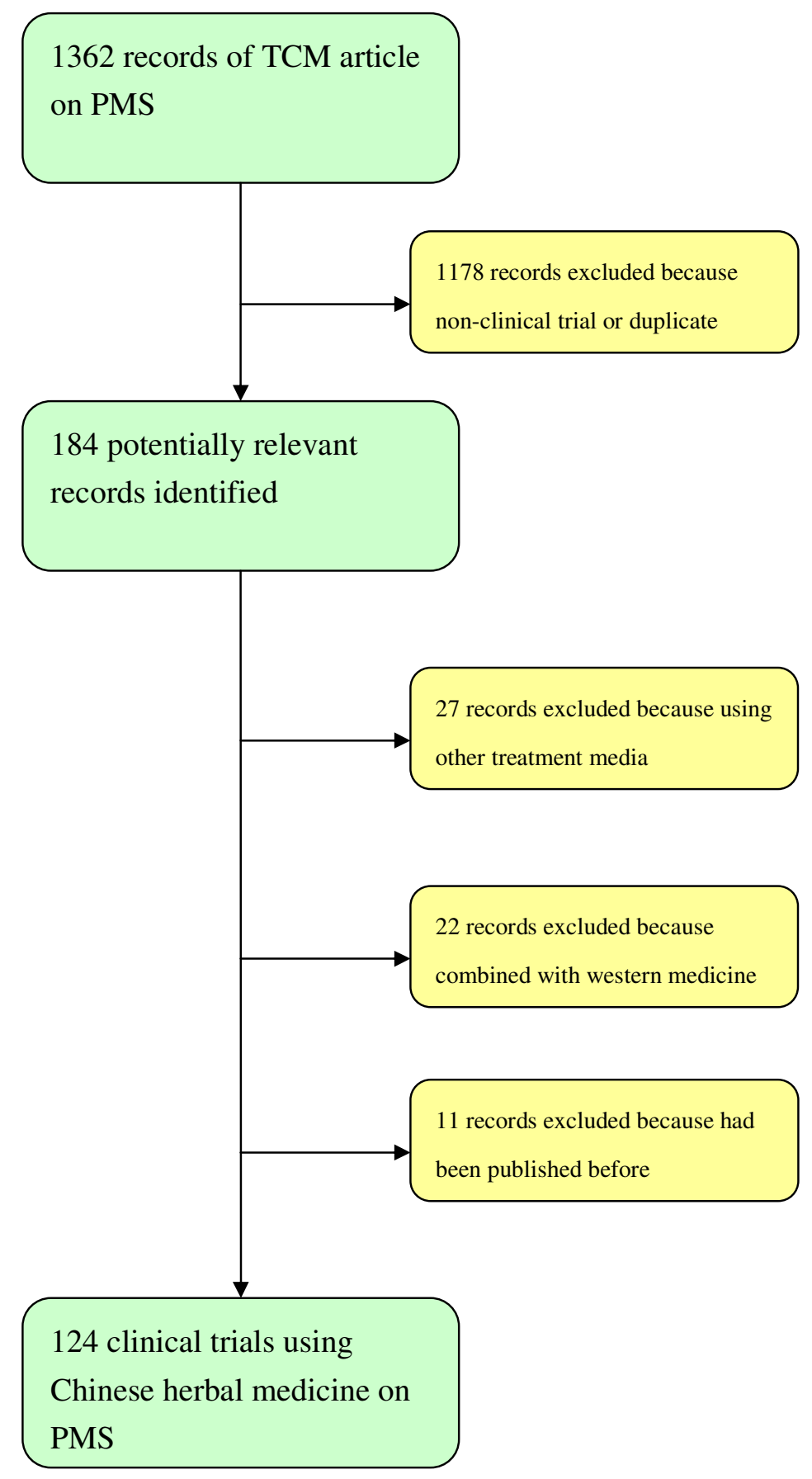

Figure 5.1 Flowchart of search results 


\subsection{Results}

\subsubsection{General description}

Following exhaustive search, 124 clinical trials (Appendix C) with a total of 10600 participants using Chinese herbal medicine for the treatment of PMS were identified. There were 40 trials on premenstrual syndrome or premenstrual tension syndrome, 48 on peri-menstrual symptoms, 31 on premenstrual symptoms, and 5 on one TCM pattern of PMS. Details of trials are shown in Table 5.1.

These trials were reported in 53 different Chinese TCM journals. Sample sizes vary from 9 to 600 . In which, the majority were between 21 to 100 participants, only 5 studies had a sample size of 300 subjects or more. Table 5.2 indicated the distribution of sample sizes. Participants in 61 trials were patients of the out-patient department, whilst participants in 6 other trials were either out-patients or in-patients of hospitals, the remaining trials neither mentioned if the participants were treatment-seeking women nor from where the participants were recruited. 
Table 5.1 Premenstrual syndrome or symptoms studied at the $\mathbf{1 2 4}$ trials

\begin{tabular}{|c|c|c|c|}
\hline \multicolumn{2}{|c|}{ Syndrome / symptoms } & No of trials & Total trials \\
\hline \multicolumn{2}{|c|}{ Premenstrual syndrome } & & 40 \\
\hline \multirow{8}{*}{ Premenstrual } & symptoms & & 31 \\
\hline & headache & 6 & \\
\hline & acne & 5 & \\
\hline & emotional disturbance & 1 & \\
\hline & sore/distended breasts & 15 & \\
\hline & insomnia & 1 & \\
\hline & swelling & 2 & \\
\hline & oral ulcer & 1 & \\
\hline \multirow[t]{12}{*}{ Perimenstrual } & symptoms & & 48 \\
\hline & headache & 18 & \\
\hline & emotional disturbance & 1 & \\
\hline & sore/distended breasts & 3 & \\
\hline & swelling & 1 & \\
\hline & oral ulcer & 7 & \\
\hline & diarrhoea & 5 & \\
\hline & coughs & 1 & \\
\hline & rashes & 3 & \\
\hline & General aches & 2 & \\
\hline & flu & 2 & \\
\hline & Sore throat & 1 & \\
\hline \multicolumn{2}{|c|}{ One TCM pattern } & & 5 \\
\hline \multicolumn{2}{|c|}{ Liver $Q i$ stagnation } & 2 & \\
\hline \multicolumn{2}{|c|}{$Q i$ and Blood stagnation } & 1 & \\
\hline \multicolumn{2}{|c|}{ Liver $Q i$ invasion } & 1 & \\
\hline \multicolumn{2}{|c|}{ Liver $Q i$ stagnation transforming into Heat } & 1 & \\
\hline
\end{tabular}


Table 5.2 Samples sizes of the 124 trials

\begin{tabular}{ll}
\hline Sample size & Trials \\
\hline$>300$ & 5 \\
$201-300$ & 4 \\
$101-200$ & 15 \\
$51-100$ & 45 \\
$21-50$ & 49 \\
$<20$ & 6 \\
\hline
\end{tabular}

\subsubsection{Definition and Diagnosis of PMS}

A definition of the disease/disorder was mentioned only in 49 out of the 124

trials. Most of them did not reference source of the definition, and there was no standardized definition of the disease/disorders.

53 trials mentioned about the standard for diagnosis of the disease, of which 2 trials diagnosed PMS according to international standard (ASA and NIMH), 11 trials followed the western medicine text books, 22 trials followed the TCM text books, and 18 trials set their own standard. Forty trials stated the inclusion and exclusion criteria of their studies.

\subsubsection{Design of the Studies}

Within the 124 clinical trials on PMS, 24 controlled studies were identified. A two group comparison design was adopted in 22 trials. One trial used a three 
group comparison, and no other types of design such as cross-over were used. Thirteen trials compared the result of their herbal medicine with a range of biomedicine including bromocriptine mesylate, estradiol, progesterone, oryzanol, natural products of soy powder, and bran skin. Two trials compared with vitamins, 8 compared with other Chinese herbal medicine, and one compared with placebo. All trials except 3 had brief descriptions of baseline characteristics. Most of them included information of age and duration of the disorder. Seven out of the 24 controlled trials did not report the significant differences between groups.

Of these 24 trials, randomization of allocation was used in 22 trials but no allocation concealment was mentioned. Double blinding was used in only 2 trials. As the traditional Chinese herbal decoctions are decocted with raw herbs, it has been a problem to conduct a controlled or double blind clinical trial for treatment with decoction. In recent years, new forms of TCM drugs such as pre-packed decoction, granules, capsules and tablets have been developed, and they render the controlled and double blind trials more feasible. In this review, only two controlled and double blind trials were identified in the past decade. One was carried in 2002 (B4 Qiao and colleagues) which examined the efficacy of herbal granules on Liver Qi Invasion of PMS and the other one was carried out in 2003 (O44 Li and colleagues) which examined the effect of herbal medicine on the depressed type PMS. B4 provided herbal granules and identical pills of other TCM herbal medicine for the control group, and O44 used identical pre-packed TCM decoction (herbal solution). However, no 
double or single blind randomized controlled trial (RCT) has been identified in 2004 and 2005.

The sizes between the treatment and control groups of most of these trials were unequal. Only 7 trials had groups of equal size. The rest of the trials used groups that were rather unequal, 7 trials with a ratio of $3: 2$, another 7 trials with a ratio of $2: 1$, and the most extreme studies had ratios as uneven as 7:2, 10:3 and 3:1.

Most trials focused on short or intermediate rather than long term outcome. Duration of the trials varied from one to six menstrual cycles/months. More than half were three-month trials only.

No details were provided of compliance, competence, or attrition of the experimental period and little or no follow-up was reported. No intention to treat analysis was mentioned.

Only a few studies provided data on the side effects of the herbal medicine. Twelve trials reported that there was no case of side effect. Only one patient in 1 trial reported minor throat discomfort and for that no treatment was needed. The rest of the trials did not mention if there was any report on side effect, or were participants questioned about any adverse effect or any new symptoms during or after treatment. 
Follow up data were available for only 6 trials. Of which, 3 trials recorded follow up data six months after completion of the treatment and the recurrent rates were $12.2 \%, 13.2 \%$ and $14.8 \%$ (Pu32-Yue and Chen 2005, Ye29-Feng and colleagues 2005 , G3-Li and colleagues 2002). In the other three trials, follow up data were recorded three months after treatment completed in Qiao's (B4 2002) report, and the follow up total effective rate only came down to 95.76\% from 96.04\%. In Lu's study (G20 2001), follow up data recorded six months after the treatment, only revealed one case of recurrence out of 25 fully recovered participants while follow up data recorded one year after treatment revealed 4 cases of relapse. In Zhang's study (Pu29 2003), only 3 out of the 12 fully recovered women had symptoms recurring 2 years after treatment. However, some researchers (51 trials) included follow up results as effective indicators for their studies.

\subsubsection{Measure of Studies}

The results of most trials were measured with subjective methods. Eighty studies rated the efficacy of the herbal medicine into three grades, i.e. full recovery, improvement (some improvement) and no effect. Thirty-one studies rated the efficacy into 4 grades i.e. full recovery, outstanding effect (substantial improvement), improvement (some improvement) and no effect. Six studies also rated the efficacy into 3 grades but differently as outstanding effect, improvement and no effect, and 7 studies only had two grading of effectiveness. 
Only fourteen trials used the changes in hormone tests (estradiol, progesterone and prolactin) before and after menstruation as a supporting measure. In 9 trials were participants asked to score their symptom severity. However, all symptom assessment charts used were individually designed. None of them was internationally or widely used, and no internal consistency or reliability of the questionnaire was mentioned

The standards for effectiveness of over $70 \%$ (91 studies) of the trials were self-designed. Eleven trials did not set any effective standard. Fifteen trials followed the criteria of therapeutic effect for peri-menstrual symptoms in the Professional Standards of Chinese Medicine, the Republic of China (Appendix B). The rest of the trials ( 7 trials) followed western textbooks. Only 11 trails had brief descriptions of their data analysis method. Twenty-nine trials reported statistical significance using p-value of their result. Clinical significance was not mentioned in any of the 124 trials.

\subsubsection{Outcome of Studies}

Ninety percent of the 124 trials reported their total effective rates. All except 5 claimed that their effective rate was over 90 percent, fifteen trials even had a $100 \%$ total effective rate. 
Out of the 24 controlled trials, effective outcome in details of the treatment and control groups were available in 22 trials. The remaining two trials only reported the significance differences between groups. Total effective rate were reported in 21 trials. The average total effective rates of the treatment and control groups of these 21 trials are $92.2 \%$ and $64.2 \%$ respectively. The total effect rates of these trials show in Table 5.3.

Table 5.3 Total effective rates of 21 trials

\begin{tabular}{lllll}
\hline & \multicolumn{3}{l}{ Total Effective Rate $(\%)$} & \\
\cline { 2 - 4 } Study & Rx & Control & Differences & P value \\
\hline Pu32 & 98.5 & 68.0 & 30.5 & $<.01$ \\
Pu20 & 90 & 52 & 38 & $<.01$ \\
Ye30 & 75 & 17.9 & 57.4 & $<.01$ \\
O30 & 97.5 & 88.3 & 9.2 & $<.05$ \\
O44 & 70 & 45 & 25 & $<.05$ \\
O19 & 98.8 & 61.3 & 37.5 & $<.001$ \\
O5 & 95.8 & 70.8 & 25 & $<.01$ \\
G3 & 98.5 & 68.0 & 30.5 & $<.01$ \\
B4 & 96.4 & 82.2 & 14.2 & $<.01$ \\
P3 & 93.3 & 53.3 & 40 & $<.01$ \\
Ye35 & 92.6 & 43.1 & 49.5 & - \\
Ye36 & 91.3 & 65.0 & 26.3 & $<.05$ \\
O25 & 83.3 & 60.0 & 23.3 & $<.05$ \\
O18 & 95.8 & 70.2 & 25.6 & $<.01$ \\
G44 & 86.0 & 58.8 & 27.2 & $<.05$ \\
Ye47 & 95.8 & 86.3 & 9.5 & $<.01$ \\
Ye48 & 92.7 & 81.1 & 11.6 & $<.05$ \\
Ye70 & 95.0 & 71.0 & 24 & $<.01$ \\
Pu44 & 95.8 & 70.8 & 25 & $<.01$ \\
Pu45 & 100 & 77.8 & 22.2 & $<.01$ \\
O63 & 94.0 & 56.7 & 37.3 & - \\
\hline & & & &
\end{tabular}


There were 1354 cases $(62.1 \%)$ with full recovery, 177 cases $(8.1 \%)$ with outstanding outcome (subatantial improvement), 542 cases $(24.9 \%)$ with some improvement, and 106 cases (4.9\%) cases with no effect out of a total 2179 cases in the treatment groups of the 22 controlled trials. The fixed-effects model of Review Manager Version 4.2.8 of the Cochrane Collaboration was used to calculate odds ratios (ORs) of outcomes between the treatment and control groups of these trials. The result revealed that the active treatment had a better ratio than the control treatment in the outcomes of 'full recovery' and 'outstanding' (Figure 5.2 and Figure 5.3), whilst the control treatment had a better ratio in the outcomes of 'improvement' and 'no effect' (Figure 5.4 and Figure 5.5). 
Review: Outcome of controlled trial of PMS

Comparison: $\quad 01$ Chines herbal medicine vs Control 01 Full recovery

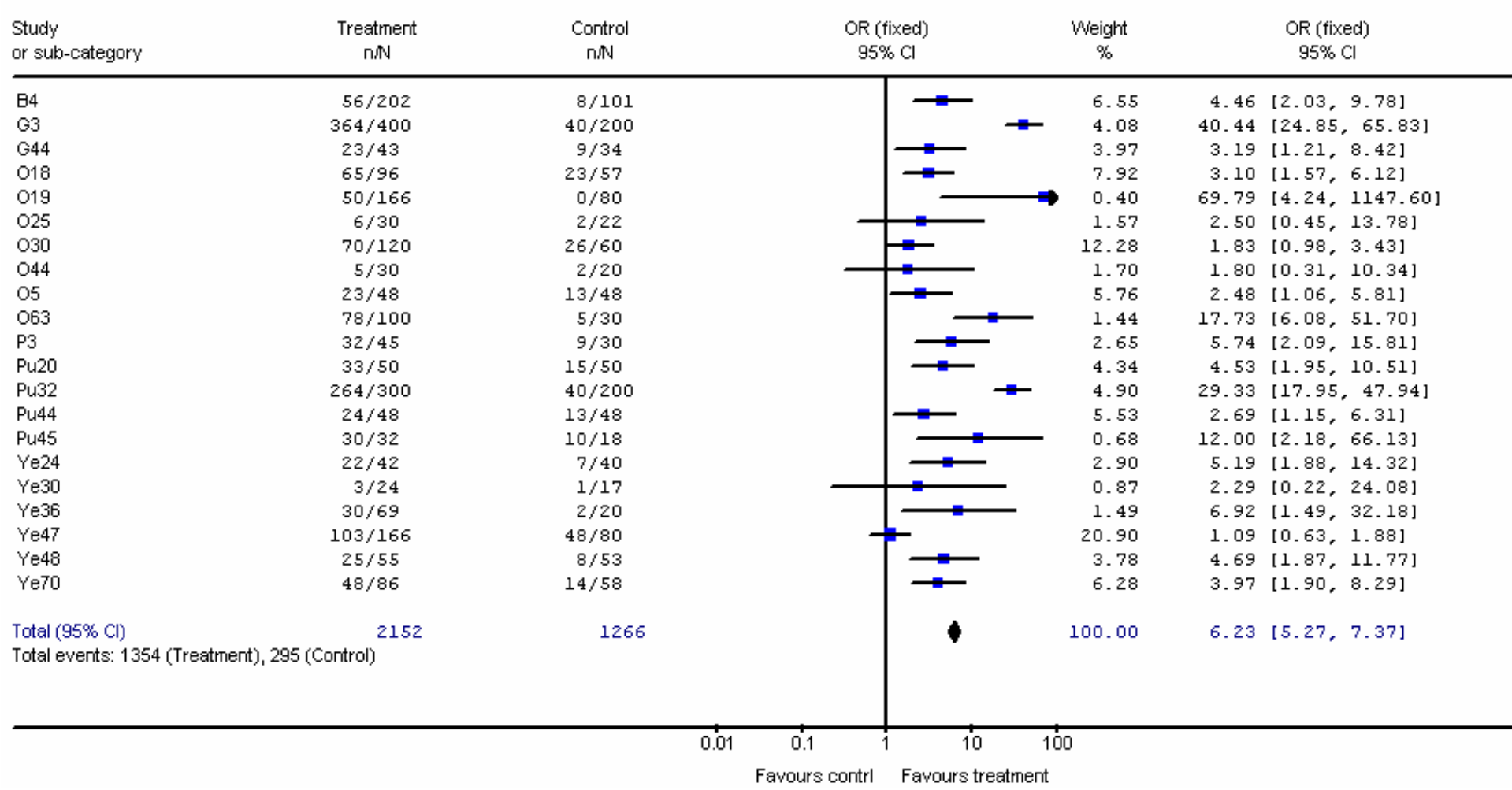

Figure 5.2 Comparison between Chinese herbal medicine and control in Full Recovery from fixed-effects model 
Review:

Comparison:

Outcome:

Outcome of controlled trials of PMS

01 Chinese herbal medicine vs control

02 Outstanding effect

Study

or sub-categor

Treatment

$n N$

84

019

044

063

P3

Pu44

Ye36

Ye48

Total $(95 \% \mathrm{Cl})$

Total events: 268 (Treatment), 112 (Control).

Test for heterogeneity: Chi $?=27.33, \mathrm{df}=8(\mathrm{P}=0.0006), \mathrm{l}=70.7 \%$

Test for overall effect: $Z=4.01(P<0.0001)$

$101 / 202 \quad 32 / 10$

$13 / 48$

$16 / 100$
$6 / 45$

\section{3}

$32 / 101$

$64 / 166 \quad 14 / 80$

$16 / 30 \quad 7 / 20$

$12 / 48$

$4 / 30$
$12 / 48$

$12 / 48$

$20 / 55 \quad 16 / 53$

$3 / 69$
$16 / 53$

101
80
20
48
30
30
48
69
53
479

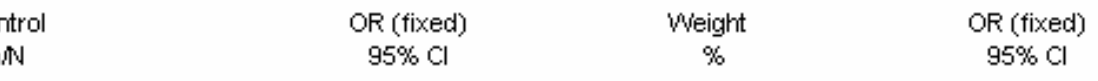

$95 \% \mathrm{Cl}$

24.64

$2.16[1.31,3.56]$

$13.41 \quad 2.96[1.54,5.70]$

$\begin{array}{rr}13.41 & 2.96 \\ 4.53 & 2.12[0.66,6.81]\end{array}$

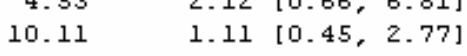

$17.91 \quad 0.29[0.12,0.71]$

$4.81 \quad 1.00[0.26,3.89]$

$10.1101 .11[0.45,2.77]$

$2.51 \quad 8.36[2.34,29.83]$

$11.98 \quad 1.32[0.59,2.95]$

100.00

$1.72[1.32,2.24]$

$0.01 \quad 0.1$

Favours control Favours treatment

Figure 5.3 Comparison between Chinese herbal medicine and control in Outstanding Outcome from fixed-effects model 
Review: Outcome of controlled trials of PMS

Comparison: 01 Chinese herbal medicine vs Conrol 03 Improvement

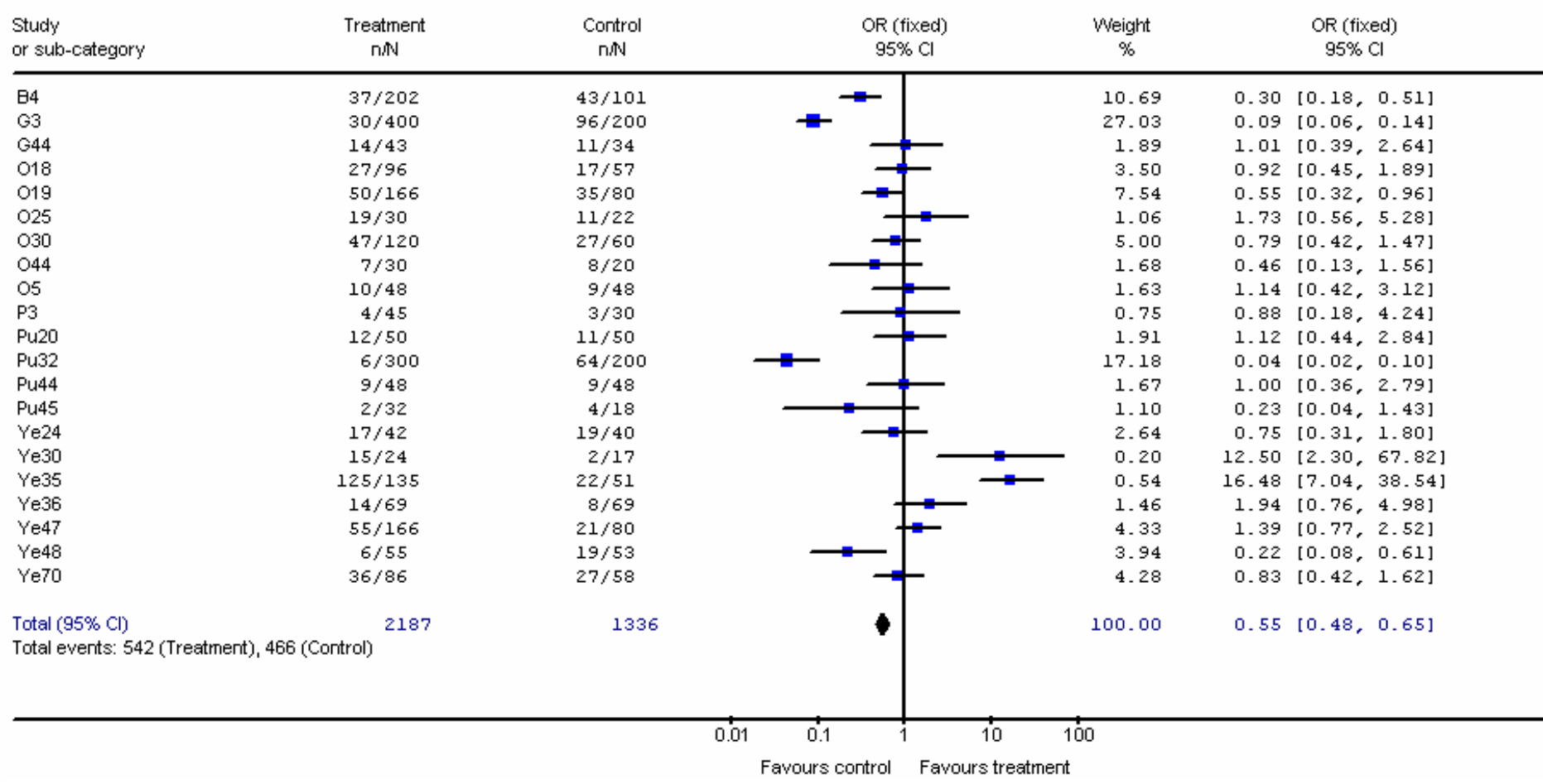

Figure 5.4 Comparison between Chinese herbal medicine and control in Some Improvement from fixed-effects model 
Review: Outcome of controlled trials of PMS

Comparison: $\quad 01$ Chinese herbal medicine vs Control

Outcome: 04 No effect

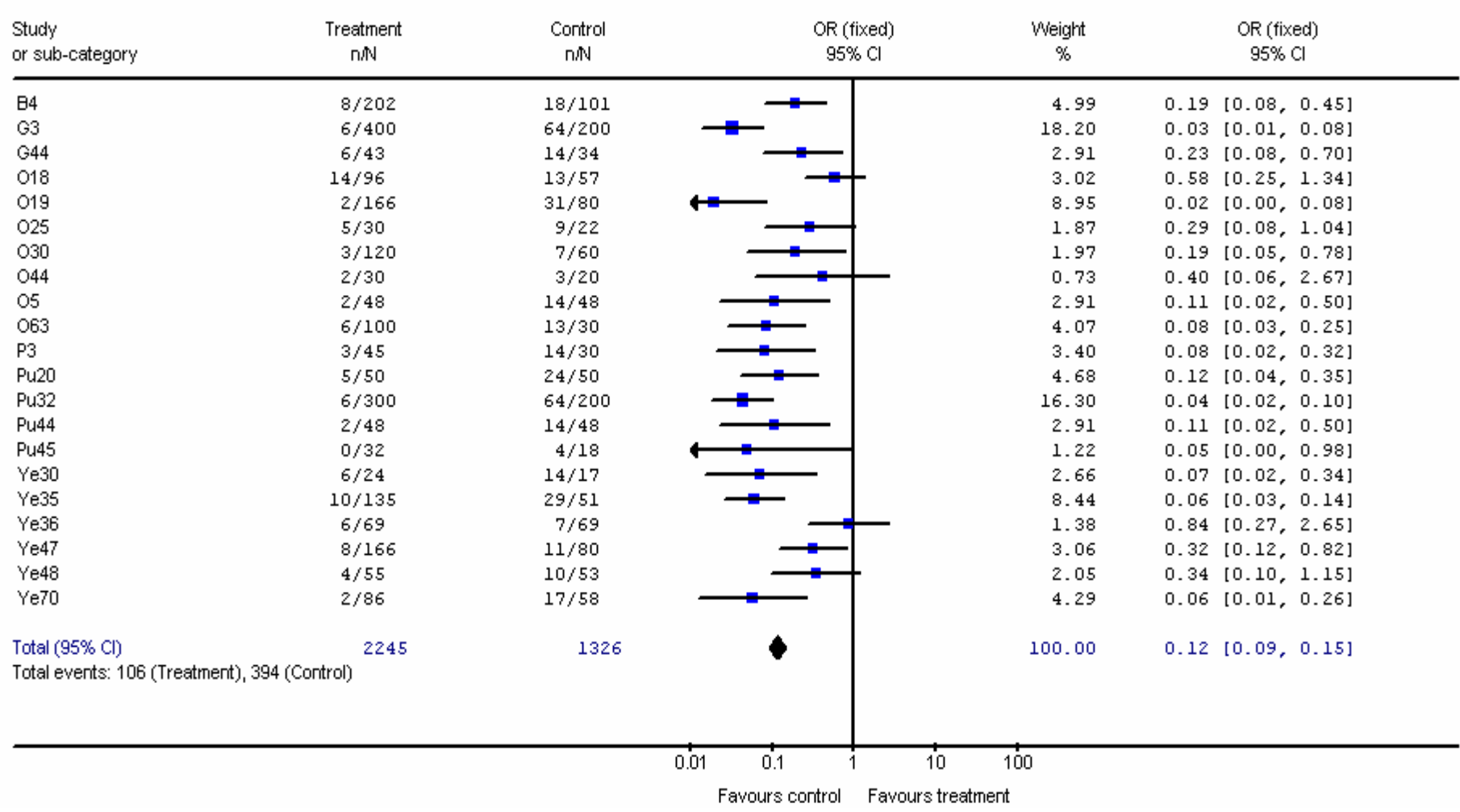

Figure 5.5 Comparison between Chinese herbal medicine and control in No Effect from fixed-effects mode 


\subsubsection{Treatment methods}

Most trials (111 studies) applied herbal medicine in the form of decoction. Seven trials used granules, 3 trials used pills, 2 trials used capsules, and one trial used herbal medicine in tablet form.

Twenty trials applied treatment according to pattern identification, of which 15 trials used modified classic herbal formulas, 1 used traditional classic formula, and 4 prescribed self-designed formulas. However, none of these trials was controlled trials. The other 104 trials used one selected formula as their basic formulas to treat the syndrome, symptoms or TCM pattern. Within these 104 trials, more than half (61 studies) used self-designed formulas, 29 used modified classic formulas, and 14 used traditional classic formulas.

In the 124 trials, a total of 41 classic formulas had been used as the basic formula. The most commonly used formulas were Xiao Yao San, Dan Zhi Xiao Yao San, Yi Guan Jian, Chai Hu Shu Gan San and Long Dan Xie Gan Tang. Table $5.4 \mathrm{t}$ to show the frequency of formulas used.

In all the trials, 53 used the set basic formula without any additional herbs, 49 trials added additional herbs to the basic formula according to symptom complaints, 17 according to TCM patterns, and 5 trials according to both symptoms and patterns. 
Table 5.4 Frequency of formulas used (more than once) in the trials

\begin{tabular}{|c|c|c|}
\hline Name & $\begin{array}{l}\text { Times used } \\
\text { in } 124 \text { trials }\end{array}$ & $\begin{array}{l}\text { Times used in } \\
45 \text { Premenstrual } \\
\text { syndrome trials }\end{array}$ \\
\hline Xiao Yao San & 16 & 11 \\
\hline (modified) & (12) & (9) \\
\hline (traditional) & (4) & (2) \\
\hline Dan Zhi Xiao Yao San & 7 & 5 \\
\hline (modified) & (6) & (4) \\
\hline (traditional) & (1) & (1) \\
\hline Yi Guan Jian- modified & 6 & 3 \\
\hline Chai Hu Shu Gan San -modified & 5 & 2 \\
\hline Long Dan Xie Gan Tang & 5 & 2 \\
\hline (modified) & (3) & (2) \\
\hline (traditional) & (2) & \\
\hline Shen Ling Bai Zhu San & 4 & 2 \\
\hline (modified) & (3) & (2) \\
\hline (traditional) & $(1)$ & \\
\hline Хие Fu Zhu Yu Tang & 4 & \\
\hline modified & (1) & \\
\hline traditional & (3) & \\
\hline Si Wu Tang -modified & 4 & \\
\hline Qi Ju Di Huang Wan -modified & 3 & 3 \\
\hline Dang Gui Shao Yao San -modified & 2 & 1 \\
\hline Gui Pi Tang -modified & 2 & 2 \\
\hline Liu Wei Di Huang Wan -traditional & 2 & 1 \\
\hline Zhi Bai Di Huang Wan -modified & 2 & 1 \\
\hline Tian Ma Gou Teng Yin -modified & 2 & \\
\hline Jian Gu Tang -modified & 2 & 2 \\
\hline Ling Gui Zhu Gan Tang -traditional & 2 & \\
\hline Si Ni San-modified & 2 & 1 \\
\hline
\end{tabular}




\subsection{Discussion and Future Directions}

Chinese medicine has used herbal medicines to treat the symptoms of the premenstrual and menstrual phases for centuries, and the results of efficacy evaluation of all these clinical trials were strongly positive. However, from this review, most clinical trials on the use of Chinese herbs to treat PMS had serious methodological flaws, and there were no rigorous clinical trials on treatment of the syndrome. The accuracy of the outstanding results is therefore questionable in the minds of many people especially among western scientists and medical practitioners.

All the 124 trials in the current critical review reported a highly positive outcome, suggesting that Chinese herbal medicine is effective for PMS. However, as most of these results suffered from unrobust experimental designs the accuracy of the claimed rates of treatment effectiveness must be viewed with caution (Smith 1975). These extremely outstanding results also call up the idea that publication bias may be present (Vickers et al. 1998). Xie and Li (1995) pointed out that publication biases may come from either the authors or the editors, and researchers and journal editors of TCM are usually not willing to publish negative results, which may reflect badly on their competence and proficiency. These findings are consistent with Gao and Chen's study (1994) which revealed that the result of the 30 studies they summarised was outstanding, but most were lacking in systematic observation and standardised diagnosis. Some of the studies were only single case reports, and very few studies had control groups. They also pointed out that all these discrepancies 
clearly affect the evaluation value of the claimed rates of treatment effectiveness. The results of Gao and Chen's review and that of the current review are similar although there has been a time lapse of 11 years between them. It is obvious that rigorous clinical trials and evidence based research are urgently needed for TCM to gain the confidence from the modern scientific world.

A true experimental study includes three properties i.e. randomisation, control and manipulation that would allow for other explanations of the phenomenon to be ruled out and thereby provide the strength of the design for testing cause-and-effect relationships (LoBiondo-Wood \& Haber 1990). A double-blinded design is generally considered appropriate to provide the most reliable data from a clinical trial (Spilker 1996). The only two double-blinded RCTs identified in this review, one was examining the efficacy of Chinese herbs on just one TCM pattern of PMS, and the other trial was just examining the depressed type of PMS.

Diagnosis and treatment application based on an overall analysis of signs and symptoms i.e. Pattern Identification is one of the basic characteristics and unique method for treatment in TCM. In this review, pattern identification was applied in less than one sixth of the trials, and none of them was a controlled trial.

For the most robust consideration, a double-blind clinical RCT with treatment based on the traditional TCM diagnosis method-Pattern Identification, is one 
highly desirable approach to scientifically evaluate the effectiveness of Chinese herbal medicine and its generalization on PMS, and to satisfy the demands of both modern Chinese and western medicine for hard, thoroughly researched evidence. 
Chapter 6: Objectives and Hypotheses of the Study

Page

6.1 Objectives of the study

6.1.1 Implications of the study

167

6.1.2 Contribution to knowledge

168

6.2 Hypotheses of the study

169 


\subsection{Objectives of the study}

The objectives of this study are:

(1) to scientifically evaluate the effectiveness of Chinese herbal medicine for the treatment of premenstrual syndrome (PMS) within the theoretical frame-work of traditional Chinese medicine (TCM),

(2) to evaluate its efficacy on Australian women in providing symptomatic relief.

(3) To provide evidenced-based data to support the treatment of PMS with Chinese herbal medicines.

\subsubsection{Implications of the study}

The study will:

- evaluate the effectiveness of Chinese herbal medicine for PMS symptoms on a sample of Australian women

- offer an alternative treatment method to western drugs for PMS sufferers who either do not accept the current treatment options or are having unwanted side-effects with the current treatment.

- add to the knowledge about the treatment of PMS for the medical profession.

- promote the application of Chinese herbal medicine in Australia

- Inform the community/ profession of the benefits that will gain from this work. 


\subsubsection{Contribution to knowledge}

1. The proposed study will address an emerging issue, that of evaluating the efficacy of a long-standing Chinese herbal medicine for a common women's health issue i.e. PMS.

2. There has been no rigorous scientific study to investigate the efficacy of Chinese herbal medicine in the treatment of PMS in either China or Australia, and the proposed study will provide a thorough evaluation of the Chinese herbal medicine in the treatment of PMS utilizing robust research methods, so this study will cover new ground.

3. The results of the study, if successful, will offer an alternative treatment method to western drugs for PMS sufferers and contribute to evidence-based knowledge about such alternative methods of treatment. 


\subsection{Hypotheses of the study}

It is hypothesized that:

1. The posttest means of the symptom occurrence and severity from the Chinese herbal medicine treatment condition will be lower than the pretest means in the same group of women.

2. The posttest means of the symptom occurrence and severity from the Chinese herbal medicine treatment group will be lower than the reports obtained from the control (placebo) group.

3. The changes in symptom occurrence and severity of PMS will be maintained at short term follow-up at three months.

4. The rating of clients' satisfaction with treatment will be positively related to treatment outcome.

No adverse effects will be anticipated as all the herbs to be used are commonly accepted and applied to a range of health problems, and are approved by the Therapeutic Goods Administration (TGA). 
Chapter 7: Methodology of the Intervention Study Using Chinese Page Herbal Medicine vs. Placebo

7.1 Design

$\begin{array}{lll}7.2 & \text { Participants } & 176\end{array}$

$\begin{array}{lll}\text { 7.2.1 Sample Size } & 176\end{array}$

$\begin{array}{lll}\text { 7.2.2 Selection Criteria } & 177\end{array}$

$\begin{array}{lll}7.2 .3 & \text { Recruitment } \text { of Subjects } & 178\end{array}$

$\begin{array}{ll}\text { 7.2.4 Diagnosis of PMS } & 179\end{array}$

$\begin{array}{ll}7.3 & 180\end{array}$

7.3.1 Analytic Approaches for Randomization of Groups $\quad 180$

7.3.2 Analytic Approaches for Menstrual Cycle Related 180 Symptoms

7.3.3 Analytic Approaches for Psychological Profiles 181

7.3.4 Analytic Approaches for Follow-up 181

7.3.5 Analytic Approaches for Statistical Significance 181

7.3.6 Analytic Approaches for Clinical Significance 183

7.3.7 Analytic Approaches for Client Satisfaction 183

7.3.8 Analytic Approaches for Adverse Effect 183

$\begin{array}{lll}7.4 & \text { Instruments } & 184\end{array}$

7.4.1 Menstrual Distress Questionnaire (MDQ) 184

7.4.2 Menstrual Symptom Diary (MSD) 185

7.4.3 Beck Depression Inventory (BDI) 186

7.4.4 State Personality Inventory (STPI) 186

7.4.5 Perceived Stress Scale (PSS) 187

$\begin{array}{lll}\text { 7.4.6 Demographic Questionnaire } & 187\end{array}$

7.4.7 Client Satisfaction Evaluation Question 187 
$\begin{array}{llr}7.5 & \text { Procedures } & 188\end{array}$

$\begin{array}{lll}\text { 7.5.1 Assessment Stage } & 188\end{array}$

$\begin{array}{ll}\text { 7.5.2 Initial Interview } & 189\end{array}$

7.5.3 Chinese Medical Consultations 189

$\begin{array}{lll}\text { 7.5.4 Treatment Application } & 191\end{array}$

7.5.5 Herbal Granules/ Placebo Preparation 192

$\begin{array}{lll}\text { 7.5.6 Data Collection } & 193\end{array}$

$\begin{array}{lll}\text { 7.6 } & \text { Ethical Approval } & 193\end{array}$

$\begin{array}{lll}\text { 7.6.1 Confidentiality } & 194\end{array}$

$\begin{array}{lll}\text { 7.6.2 Potential Risks } & 194\end{array}$ 


\subsection{Design}

The present research was a double-blind randomized placebo-controlled crossover clinical trial conducted by a qualified Chinese herbal practitioner and pharmacist. In order to assign the participants randomly into two groups of the crossover trial within different TCM (traditional Chinese medicine) patterns, the randomized block design was used.

On the basis of the information gathered, the researcher/practitioner made a diagnosis of the TCM pattern of disharmony of each participant. The woman was then grouped into one of the most common premenstrual syndrome (PMS) pattern subgroups:- namely, Patten A (Liver $Q i$ stagnation), Pattern CA (Liver and Kidney Yin deficiency), Pattern H (Liver \& Spleen disharmony), Pattern D (Heart \& Spleen deficiency) and Pattern O (any other patterns). In each of these pattern-groups, sequential allocation (Appendix D) to Group I or Group II was carried out. Thus the treatment effect within a subgroup/TCM pattern could be assessed (Figure 7.1), and with the pooled data of the subgroups, an overall effect could be assessed (Figure 7.2).

Herbal medicine and placebo were provided to the subjects in both groups for a period of three months each. The participants in Group I received herbal medication for the first three months and placebo for the following three months. The participants in Group II received placebo for the first three months and herbal medication for the following three months (see Figure 7.3). The 
randomization list was kept and the treatment condition allocation was carried out by the herbal pharmacy staff. They were instructed to keep the list private and to only reveal a treatment allocation after receiving information demonstrating that the participant was eligible and had consented to the trial. Both the investigator/TCM practitioner and the subjects did not know whether the participants were assigned to Group I or Group II. The researcher was only informed of the treatment allocation after the study. Data collected before, during and after the study were statistically analysed to evaluate the efficacy of Chinese herbal medicine on these Australian women with PMS.

Data used for the analyses were taken at each luteal phase at baseline assessment prior to randomization into a group, at each of the three cycles of herbal and placebo treatment of Groups I and Group II, and at the three months follow-up after completion of treatment. 


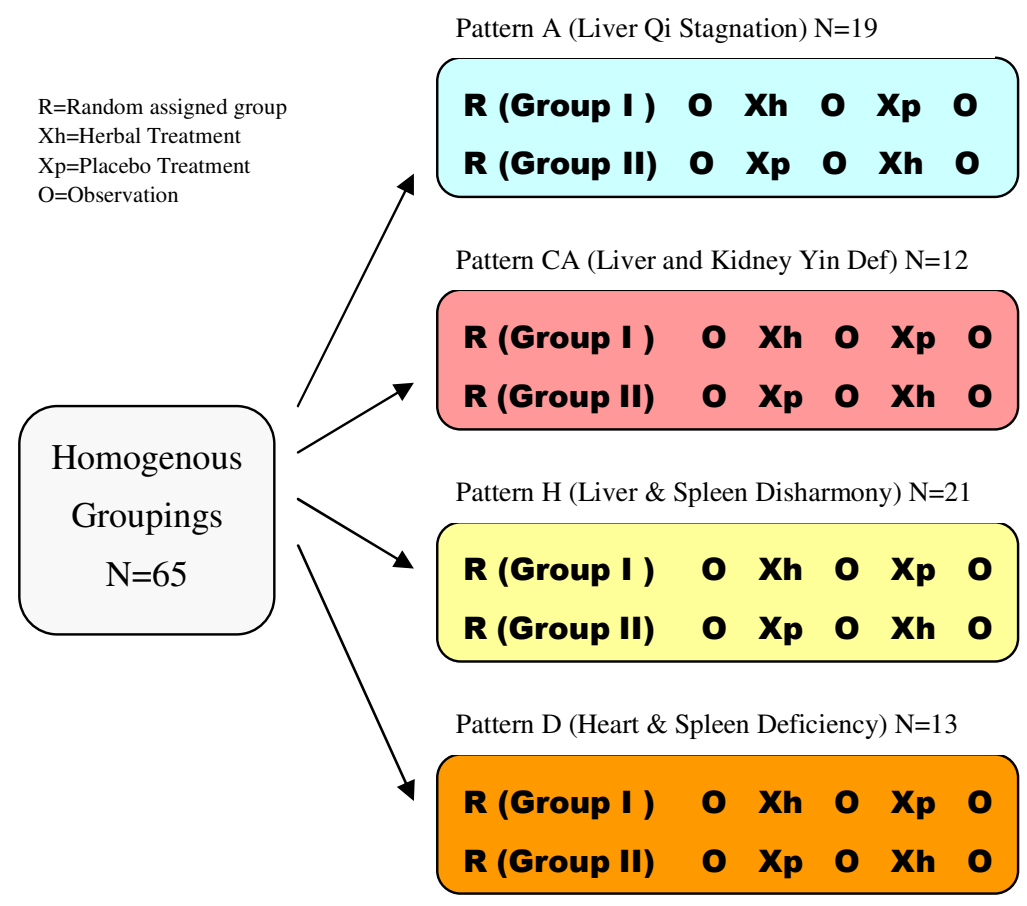

Figure 7.1 Subject Pattern assignment flow diagram 1

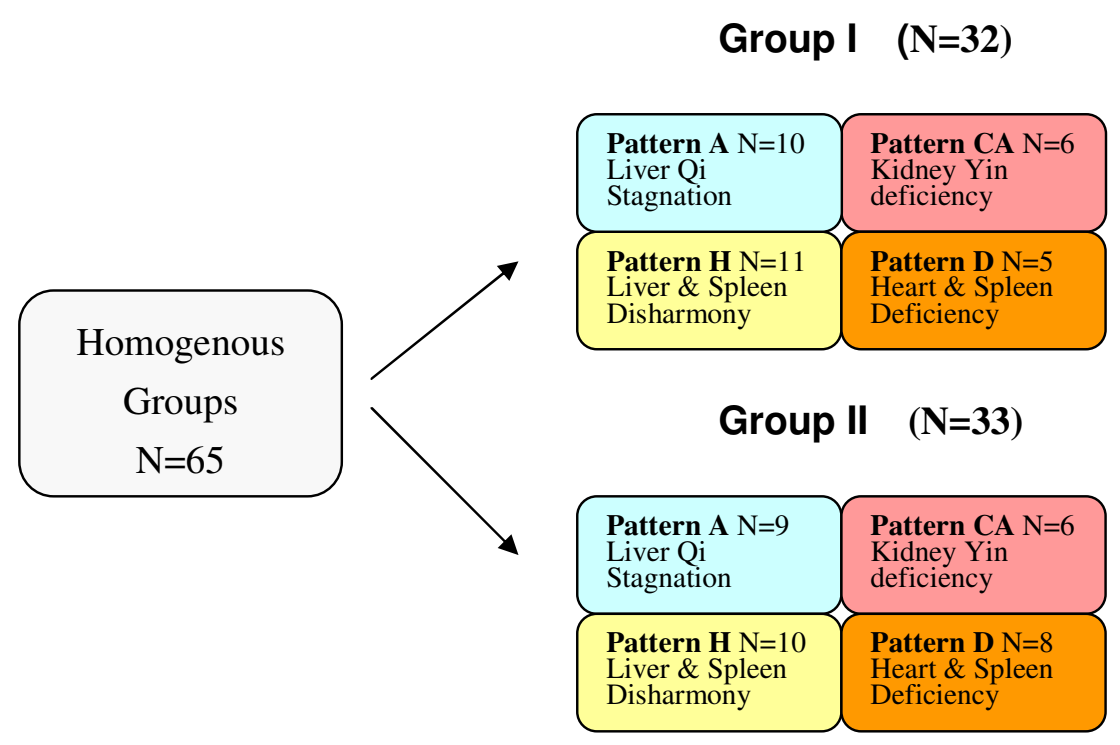

Figure 7.2 Subject Pattern assignment flow diagram 


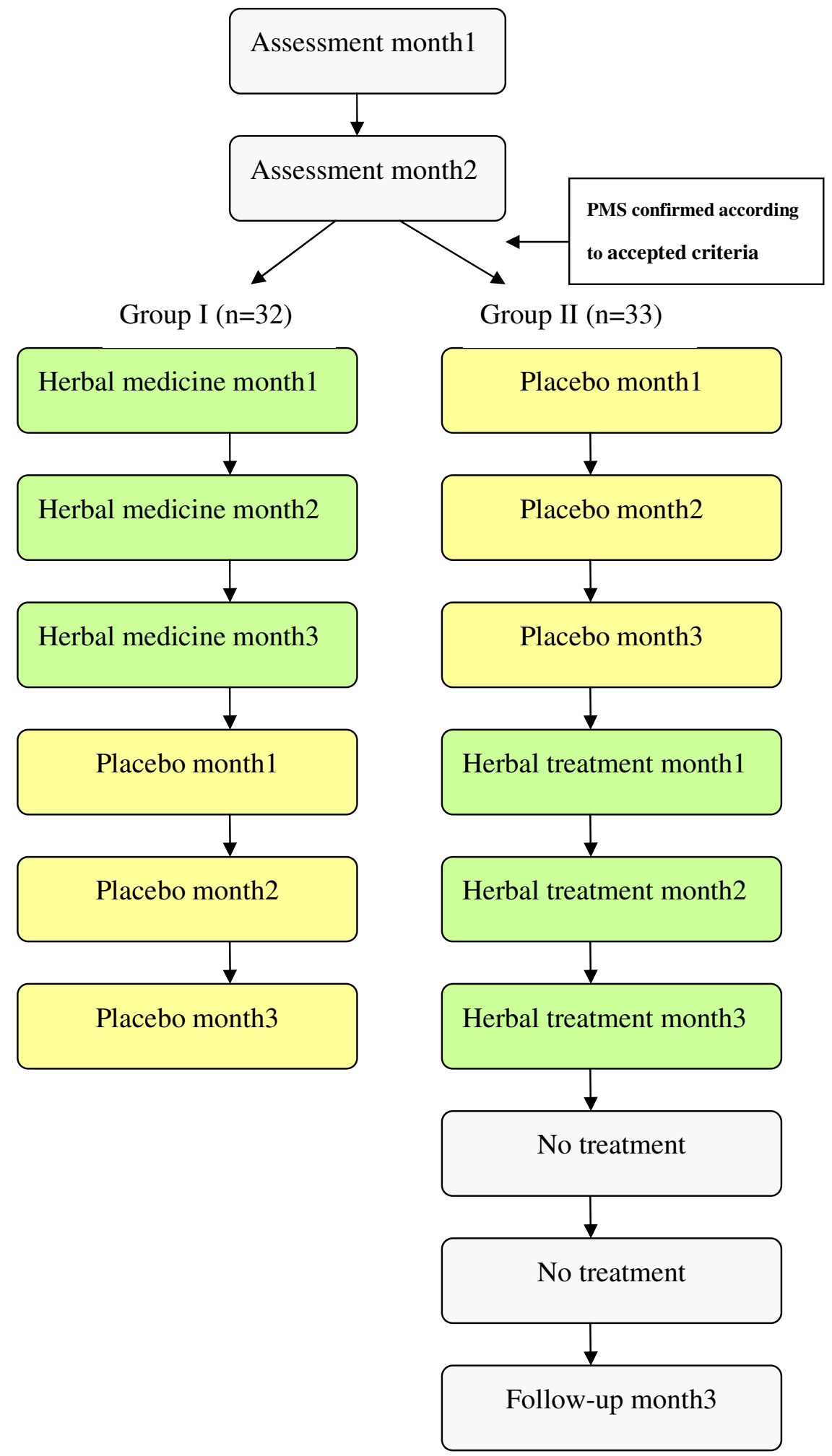

Figure 7.3 Study Timeline 


\subsection{Participants}

\subsubsection{Sample size}

Power analysis using Power and Precision (Borenstein et al. 2000) was carried out to ascertain the minimum number of subjects required for each group. A sample size of 33 subjects in each treatment group is required to provide a power of $80 \%$, in order to detect a medium clinical effect size (0.3SD) at a significance level of 0.05 for the primary outcome measures of the Menstrual Distress Questionnaire (MDQ) symptom scores.

One hundred and fifty two women were recruited who then joined the assessment phase of the study. Five women did not meet the inclusion criterion that at least $30 \%$ increase in the MDQ scores during the pre-menstrual phase as compared to the post-menstrual phase. However, due to the high attrition in the assessment period, only sixty-five treatment seeking women with premenstrual symptoms remained and participated in the actual experiment. Within the 82 withdrawals (drop-out rate 54\%), 36 could not keep records due to forgetfulness; 14 did not want to wait and required immediate treatment; 10 could not be reached; 5 were to too busy; 6 due to personal reasons, 5 developed other medical problems, 4 had little or no symptom and 2 due to moving. The final numbers were 32 women in Group I, and 33 in Group II. Among them, 19 were in Pattern A, 12 in Pattern CA, 21 in Pattern H and 13 in Pattern D (see Figure 7.2). 


\subsubsection{Selection Criteria}

A number of inclusion criteria were applied to ascertain women's eligibility to take part, drawn from internationally agreed standards (WHO 1992; APA 1994; ACOG 2000; NIMH cited in Parker 1994) to confirm the presence of the condition: a) The woman has suffered PMS symptoms for at least the prior six months. b) The premenstrual complaints must occur in the luteal phase, resolve within one or two days after the onset of menstrual bleeding, and have a symptom-free period in the follicular phase. c) These complaints must be recurrent and severe enough to affect a woman's normal lifestyle. d) The PMS symptoms must not be caused by any other physiologic or psychological disorder. e) Disorders such as major depression, anxiety, hypothyroidism, and diabetes must be excluded. f) PMS diagnosis is dependent on the occurrence of an increase in symptom severity of at least $30 \%$ in the seven days before menstruation as compared with the days after menses. g) These symptoms must be present during at least two consecutive cycles.

The present research was open to Australian women residing in Melbourne who met the following selection criteria:-

1. have a history of premenstrual complaints for at least six months

2. be aged 18 or over

3. have been living in Australia for over two years

4. have regular menses, and is not pregnant or lactating

5. be physically healthy

6. have no gynaecological or endocrine illness 
7. have no history of psychiatric disorders

8. have no chronic medication for at least 6 months

9. have no current use of oral contraceptives, or any other hormones, antidepressants, tranquillisers, diuretics, hypertension medicine or corticosteroids

10. experience at least $30 \%$ increase in symptom scores during the pre-menstrual (late-luteal) phase as compared to the post-menstrual (mid-follicular) phase demonstrated in the prospective assessments over two consecutive months, and a minimum 30 score points of the MDQ at the premenstrual phases.

\subsubsection{Recruitment of Subjects}

Recruitment of treatment seeking women was carried out with advertisements being placed through various means to maximise the number of respondents.

1. Recruitment news releases were published in the national newspapers, Women's Health Victoria, Chinese Medical Associations' newsletters (Australian Acupuncture \& Chinese Medicine Association; Federation of Chinese Medicine and Acupuncture Societies of Australia) and seven local newspapers.

2. Advertisements (Appendix E) were also placed on noticeboards in the health clinics of the University and around the campuses of other universities in Melbourne. 
3. Recruitment fliers (Appendix F) were placed at community health centres, private Chinese medical clinics, conferences, seminars, and the Australian Complementary Health Association.

4. Electronic recruitment messages were also sent to all staff and research students through the Unimail Global Message System of the University.

Due to the high drop-out rate in the assessment period, recruitment of subjects was slower than originally planned and the above strategies had to be repeated three times.

\subsubsection{Diagnosis of PMS}

The mean scores of all daily symptoms of the participant were recorded on the menstrual symptom diary (MSD) during the premenstrual phase (the seven days before menstruation) and the follicular phase (the seven days following the cessation of menses) for two consecutive cycles. The mean severity scores of the three most severe days of symptoms were assessed for both the premenstrual and post-menstrual phases. A minimum of $30 \%$ increase of the mean score in the premenstrual phase was required compared with the postmenstrual phase on the MSD (Parker 1994). A minimum of 30 points

difference in the Menstrual Distress Questionnaire comparing premenstrual and follicular phases were required to fulfil the recommended inclusion criteria. 


\subsection{Analytic Approaches}

\subsubsection{Analytic Approaches for Randomization of Groups}

The demographic characteristics and baseline levels of composite physical and psychological menstrual symptoms from MDQ, depression, state-anxiety, state-anger, and perceived stress of the women in Group I and II were compared, using MANCOVA (multiple analysis of covariance), to ascertain whether randomization of groups was successful, i.e. there was no difference at entry was found between the two groups.

\subsubsection{Analytic Approaches for Menstrual Cycle Related Symptoms}

Data collected from the MDQ on Day 2 of the cycle covering their experiences in the week before menstruation (premenstrual phase) and the mean score on each of the symptoms across the seven days before menstruation in the MSD were used for statistical analysis. The between-subjects and within-subjects differences of herbal and placebo treatment condition in the scores of the physical symptoms and psychological symptoms were measured and compared using repeated measures ANOVAs (analysis of variance) with Bonferroni adjustments (1) at baseline and during the first three months of Group I and Group II and (2) at baseline and all six treatment months of Group II. 


\subsubsection{Analytic Approaches for Psychological profiles}

The changes in psychological profiles i.e. depression (BDI), anxiety (ANX), anger (ANG) and perceived stress (PSS ) were also assessed repeatedly using data collected from the Beck Depression Inventory, Spielberger's State-Trait Personality Inventory and Perceived Stress Scale. The same analysis procedure and tests as for the analysis of MDQ and MSD were performed on BDI, ANX, ANG and PSS.

\subsubsection{Analytic Approaches for Follow-up}

The score differences of the physical symptoms and psychological symptoms before herbal treatment, during herbal treatment condition and three months

after the completion of the experimental period were analysed using repeated measure ANOVAs with Bonferroni adjustments of Group II, those whose second treatment condition was the active constituent.

\subsubsection{Analytic Approaches for Statistical Significance}

By using the raw scores of the symptom severity as the dependent variables, and treatment as the independent variable, SPSS (Statistical Package for the Social Sciences) was utilized for statistical analysis of data. All data were coded. Descriptive analysis of all variables was carried out. Univariate or 
multivariate analysis using repeated measure MANOVA (multivariate analysis of variance), ANOVA and/or independent t-test were performed. Pairwise comparisons were undertaken to compare all different combinations between times of the treatment condition/groups. Bonferroni adjustments were used for pairwise comparisons to control type I error. The conventional alpha level of .05 was used for all statistical tests.

Prior to statistical analysis, all variables were examined through various SPSS program 11.0, (2001) for accuracy of data entry, normality, missing values, and fit between their distributions and the assumptions for multivariate analysis. There were very few random missing data on the clinical assessment scores that included the MDQ, BDI, STPI and PSS where the subjects failed to provide some information on certain questions. Mean substitution was performed when less than $5 \%$ of data was missing for the clinical assessment scores. Missing data in the follow-up using the MDQ revealed 14.8\% (n=9) of the subjects in Group II did not return the questionnaire at the short term assessment three months after treatment. Deletion of cases pairwise was carried out when the follow-up data were analysed. Only 29 women in total had returned and completed their MSD during the premenstrual phase at the herbal treatment periods. As a result, only the data of these 29 subjects were used for the analysis of individual premenstrual symptoms. 


\subsubsection{Analytic Approaches for Clinical significance}

The clinical significance of successful clinical treatment outcome was examined using combined data collected from the MDQ, BDI, STPI and PSS. The clinical changes were measured for the reduction of scores, the percentage of improvement within subjects and the effect sizes after herbal and placebo treatments.

\subsubsection{Analytic Approaches for Client satisfaction}

The relationship between client satisfaction and the treatment outcome was examined. An evaluation question eliciting client satisfaction will be constructed as a 5-points likert scale where $1=$ Not at all satisfied and 5= Very satisfied. Clients will be asked to respond to this item at the completion of the herbal treatment condition and the placebo treatment condition. The clients' satisfaction ratings were compared with the treatment scores on PHYS (composite scores of physical symptoms), PSYC (composite scores of psychological symptoms), BDI, ANX, ANG and PSS using Kendall's Tau-b correlation coefficient at the third and sixth months of the study.

\subsubsection{Analytic Approaches for Adverse effect}

Any adverse effects or complaints were invited but none was reported. 


\subsection{Instruments}

The treatment outcome between the pretest, the posttest, and the after effect of both herbal and placebo medicine of the study were measured by the occurrence and severity of the premenstrual physical and psychological symptoms using the following instruments.

\subsubsection{Menstrual Distress questionnaire (MDQ)}

In the present study, Form T of the revised version of Moo's MDQ (1985) was used to repeatedly assess the symptoms occurrence and severity of PMS. Participants were asked to complete the MDQ on Day 2 of their menstrual cycle to rate their experiences the week before the period of each of the 47 symptoms listed in the form.

Scores of the symptom clusters on the MDQ were separated into physical and psychological symptom groups for analysis. Since Moos (1968) suggests that the Arousal and Control scales do not appear to be particularly reflective of cyclical changes related to the menstrual cycle, the mean scores on the Arousal and Control scales at premenstrual and postmenstrual periods of the women in the present study at their first assessment month were analysed using Paired Samples t-test, and there is no significant difference between the two time periods on both scales. The Arousal and Control symptoms were not included 
for the present analysis. The remaining six symptom clusters were separated into physical and psychological symptom groups. Scores of the Pain, Water Retention and Autonomic Reaction symptom scales on MDQ were combined together and named as Physical symptoms (PHYS), whilst scores of the Concentration, Behavioral Change and Negative Affect symptom scales were combined together and named as Psychological symptoms (PSYC). (A copy of the MDQ is attached at Appendix G).

\subsubsection{Menstrual Symptom Diary (MSD)}

The Abraham's Menstrual Symptom Diary (1980) was used for recording menstrual symptoms prospectively in the study. The titles of the cluster categories in the form were deleted to minimize confusion.

Participants were asked to start rating their symptoms from the first day of their cycle, and to rate each symptom every evening referring to the presence or absence and the intensity of the symptom in the previous 24 hours. The mean severity score was then obtained for seven post-menstrual days and for seven premenstrual days. The mean of the three most severe days was calculated for both the premenstrual and post-menstrual phases, and a mean difference score was determined. A copy of the MSD is printed in Appendix H. 


\subsubsection{Beck Depression Inventory (BDI)}

The short form Beck depression inventory (1972) of 13 items was used to measure the depressive symptoms of the women in the study. Each of the items is rated on a 4-point Likert scale (range 0-3). Participants were asked to circle the number next to the particular statement for each symptom which best describes the way they had been feeling the week before the last period. The total score of the BDI was obtained by adding the scores of all items. A copy of the BDI is attached in Appendix I.

\subsubsection{State Personality Inventory (STPI)}

The state-anxiety (1979) and state-anger subscales (1979) were combined and named as STPI in the present study. It was used to assess the level of anxiety and anger of the women at their premenstrual period. Participants were asked to read the statement and then circle the appropriate grade for each of the 20 items on the form to indicate how they had felt in the week before the last period. Each of the items is rated on a 4-point Likert scale (range 1-4). (see Appendix J) 


\subsubsection{Perceived Stress Scale (PSS)}

The perceived stress scale (Cohen, Kamarck \& Mermelstein 1983) was used in the study to measure the level of psychological distress. Participants were asked to rate their reaction on the 14 items and a 5-point Likert type scale (range 0-4) of the form monthly during the study. (see Appendix K)

\subsubsection{Demographic Questionnaire}

Demographic variables of interest included age, marital status, occupation, employment, education, income, race and attitude towards TCM were assessed by single item questions on the questionnaire. (see Appendix L)

\subsubsection{Client satisfaction evaluation question}

Clients were asked to respond to an evaluation question eliciting client satisfaction, ratings according to a 5-points likert scale where $1=$ Not at all satisfied, 2= Somewhat, 3= Moderately, 4= Quite and 5= Very satisfied, at the completion of the experimental period and the placebo period. 


\subsection{Procedures}

\subsubsection{Assessment Stage}

Following a telephone contact when primary screening of basic criteria was conducted and the study was briefly explained, prospective participants who met the inclusion criteria 1-9 were requested to prospectively complete a menstrual symptom record for two consecutive menstrual cycles. An Information Statement for Participants (Appendix M) which explains the project and an Informed Consent Form (Appendix N), 3 copies of the MSD and the MDQ, an envelop with returning address and an instruction sheet were posted to prospective participants. The prospective participants were asked to read the information, complete the forms as instructed, and return the completed forms to the researcher by post with the attached envelop.

Prospective participants who completed the diaries and whose symptom profiles worsened/ameliorated by $30 \%$ during the premenstrual period in comparison to the post menstrual period were accepted into the trial. Formal interviews and assessments including a Chinese medical consultation were arranged to take place at the post-menstrual phase during cycle days 5-12. 


\subsubsection{Initial Interview}

At the initial interview, information was provided concerning the aims, nature and procedures of the study, the nature of the Chinese herbal medicine to be used, any potential adverse effects that might occur, confidentiality with regard to the identity and personal information of the subjects, freedom to ask questions, the right to withdraw at any time, and all risks and safeguards of the project were explained to all prospective participants. Demographic data, medical and psychosocial history, and PMS symptoms were collected during the interview after written informed consent had been obtained. A Chinese medical consultation was then carried out after all the paper work had been processed.

\subsubsection{Chinese medical consultations}

A Chinese medical consultation was performed by the investigator, a qualified Chinese herbal practitioner, in the Health Clinic of the University for the convenience of the participants. During the consultation, participants were asked about their past health histories, chief symptoms, duration of PMS symptoms, menstruation, vaginal discharges, parity histories, diet and digestion, bowel and urine, sleep, emotion, energy, fluid, cold/hot sensation, discomfort and pains. Each participant's general appearance, spirit, facial complexion and tongue condition were also directly observed, and the radical pulses were taken. All the information was recorded in the TCM Consultation Form. The 
investigator, on the basis of the information gathered through the four methods of TCM diagnosis (see Subdivision 3.4.1), a diagnosis of the TCM pattern of disharmony of each participant was made, and each woman's condition was then grouped into one of the patterns of disharmony of PMS (see Table 7.1). Independent assessments were carried out by another qualified TCM practitioner at the initial and final consultation to gauge outcomes and provide quality assurance

Table 7.1 Signs and symptoms of TCM patterns of disharmony of PMS

\begin{tabular}{|c|c|c|}
\hline Pattern of Disharmony & Signs and Symptoms & TCM diagnosis \\
\hline \multicolumn{3}{|l|}{ Pattern A } \\
\hline Liver Qi Stagnation & $\begin{array}{l}\text { Anxiety, irritability, depression, sadness, mood } \\
\text { swing, agitated, headache, breast tenderness, } \\
\text { hypochondrian pain, sighing, period pain, }\end{array}$ & $\begin{array}{l}* \mathbf{P} \text { - wiry } \\
* \mathbf{T} \text { - normal or dull } \\
* \mathbf{M} \text { - irregular, dark red with } \\
\text { small clots }\end{array}$ \\
\hline $\begin{array}{l}\text { Liver Qi Stagnation } \\
\text { transforming into Fire }\end{array}$ & + bitter mouth, dry throat, red eyes & $\begin{array}{l}\text { P- wiry and rapid } \\
\text { T- red sides, yellowish coating } \\
\text { M- profuse, thick, dark red }\end{array}$ \\
\hline \multicolumn{3}{|l|}{ Pattern CA } \\
\hline $\begin{array}{l}\text { Liver Qi Stagnation } \\
\text { Liver and Kidney Yin } \\
\text { Deficiency causing heat } \\
\text { in Internal Organs }\end{array}$ & $\begin{array}{l}\text { Same as Pattern A } \\
\text { +head ache, dizziness, tinnitus, oral ulcers, } \\
\text { acne, red eyes, feverish sensation, increase } \\
\text { appetite, cravings, malar flush, dry throat and } \\
\text { mouth, insomnia, nocturnal sweating, sore } \\
\text { back, constipation }\end{array}$ & $\begin{array}{l}\text { P-wiry, thin and rapid } \\
\text { T-red, thin and yellow coating } \\
\text { M-early, profuse, bright red } \\
\text { with small clots }\end{array}$ \\
\hline \multicolumn{3}{|l|}{ Pattern $\mathbf{H}$} \\
\hline Liver Qi Stagnation & Same as Pattern A & \\
\hline $\begin{array}{l}\text { Liver \& Spleen } \\
\text { Disharmony } \\
\text { Spleen Deficiency causing }\end{array}$ & $\begin{array}{l}\text { +fluid retention, swollen of limbs, loose stool, } \\
\text { fatigue, abdominal bloating, reduce appetite, } \\
\text { breast distension, hypersomnia, weight gain }\end{array}$ & $\begin{array}{l}\text { P- slippery or soft } \\
\text { T- pale, fat, with teeth marks } \\
\text { M- light in colour }\end{array}$ \\
\hline \multicolumn{3}{|l|}{ Dampness Retention } \\
\hline \multicolumn{3}{|l|}{ Pattern D } \\
\hline Liver Qi Stagnation & Same as Pattern A & \\
\hline Heart \& Spleen & + depression, crying, forgetfulness, difficulty & P- thin \\
\hline Deficiency & $\begin{array}{l}\text { concentrating, confusion, insomnia (disturbed } \\
\text { sleep), palpitation, reduce appetite, lethargy, } \\
\text { abdominal bloating }\end{array}$ & $\begin{array}{l}\text { T- pale, thin white coat } \\
\text { M- pale and thin }\end{array}$ \\
\hline \multicolumn{3}{|l|}{ Pattern O } \\
\hline Any other patterns & According to different patterns & \\
\hline
\end{tabular}




\subsubsection{Treatment Application}

Participants within each pattern of disharmony were assigned randomly either to Group I or Group II. The participants in Group I received herbal medication for the first three months and placebo for the following three months. The participants in Group II received placebo for the first three months and herbal medication for the following three months. Herbal or placebo treatment for individual participants was prescribed according to their TCM patterns of disharmony (Table 7.2). Prescriptions for individual participants therefore differed in combination and strength of herbs. Herbal medicine or placebo $10 \mathrm{~g}$ per day, the recommended standard dose of herbal granules (Sun Ten 2001), was given for a duration of 10 days before each menstruation for three consecutive cycles. No information was provided to the participants where taste was expected to differ between herbal granules and placebo. The taste may change as different herbs were used in different formulas during the course of study.

\section{Table 7.2 Treatment for TCM patterns of disharmony}

\begin{tabular}{llll}
\hline Pattern & Pattern of Disharmony & Treatment Principle & Herbal Medicine \\
\hline A & $\cdot$ Liver Qi Stagnation & Soothe Liver & Modified Xiao Yao San \\
& $\cdot$ Liver Qi Stagnation & Regulate Liver Qi & +/- YuJin, GouLou, \\
& transforming into Fire & Resolve Stagnation & \\
& & +Clear Heat & Modified Dan Zhi Xiao Yao San \\
CA & $\cdot$ Liver Qi Stagnation & Nourish Yin & Modified Xiao Yao San \\
& $\cdot$ Liver and Kidney Yin & Clear Heat & +/- DanPi, ZhiZi, JuHua, YuZhu, \\
& deficiency causing heat in & Regulate Liver Qi & MaiDong, Shang/ShouDiHuang, \\
& Internal Organs & & \\
& $\cdot$ Liver Qi Stagnation & Strengthen Spleen & Modified Xiao Yao San, \\
& $\cdot$ Liver \& Spleen Disharmony & Remove Dampness & + Shen Ling Bai Zhu San \\
& $\cdot$ Spleen Deficiency causing & Soothe Liver & +/- GuiZhi, ZhuLing, ZheLan \\
& Dampness Retention & Regulate Liver Qi & \\
& $\cdot$ Liver Qi Stagnation & Tonify Spleen & Modified Xiao Yao San, \\
& $\cdot$ Heart \& Spleen Deficiency & Nourish Heart & + Gui Pi Tang \\
& & Soothe Liver & +/- FuXiaoMai
\end{tabular}




\subsubsection{Herbal granules/ placebo preparation}

Herbal granules were used in the study. The herbal granules which were concentrated herbal extracts manufactured through modern technology were imported from GMP (Good Manufacturing Practices) Chinese herbal suppliers of Taiwan. The granules had been tested by an Atomic Absorption Spectrophotometer to ensure that they were free of heavy metal and pesticide residues, and were monitored through a High Performance Liquid Chromatograph Quantitative Analysis to ensure a consistent concentration of active ingredients. All the herbs used in the study were commonly accepted and applied to a wide range of health problems, and were approved by the Therapeutic Goods Administration (TGA), Australia. The placebo used was made from baked corn starch with a small amount of added liquorices powder (1\%) which was identical in appearance to the herbal granules in size, shape and colour, and had a bitter and liquorices taste. Corn starch is commonly used as binder for the manufacture of herbal granules, and liquorices has a neutral nature and a harmonizing action in TCM. So the placebo made by these ingredients should have no adverse action on PMS symptoms. All the herbal medicine used in the study was prepared by a qualified TCM pharmacist. 


\subsubsection{Data Collection}

Demographic data, medical and psychosocial history, and PMS symptoms were collected during the first interview. Each participant was also interviewed and received a Chinese consultation by the investigator every month during her post menstrual phase from pre-treatment to end of the $2^{\text {nd }}$ treatment condition. Participants in Group I were requested to chart a menstrual diary recording their menstrual symptom every cycle, and to complete the MDQ, BDI, STPI, and PSS during the week before their last period at Day 2 of their cycles for the whole course of the study to provide data on negative moods, symptoms and concurrent stress. Group II subjects were requested to do the same, and at three months after the completion of herbal treatment to provide follow up data. All participants were asked to respond to an evaluation questionnaire eliciting client satisfaction at the completion of the experimental period and the placebo period.

\subsection{Ethical Approval}

Formal approval for the study to proceed was obtained from the Human Research Ethic Committee of Victoria University in 2001. 


\subsubsection{Confidentiality}

All information and data collected from the questionnaires and during the TCM consultations were kept in a locked filing cabinet. Codes were used to identify participants. The list of names and codes was kept in another locked filing cabinet separate from the data. Only the investigators knew the names of the participants. Ethical guidelines set out by the National Health and Medical Research Council (NH\&MRC 1992; 1999) were strictly followed. No person other than the investigators had access to the data. All data will be destroyed five years after the completion of the study following publication of data.

\subsubsection{Potential Risks}

No physical adverse effect was anticipated as all the herbs or herbal formulas used in this study were commonly accepted and applied to a wide range of health problems, and the herbal granules to be used were approved by the Therapeutic Goods Administration (TGA) of Australia. They were free of heavy metal and pesticide residues. No animal products or any endangered species were used in this study. Whilst it is unusual for any participant to experience any allergic effect from the herbal granules, the most possible symptoms of allergies that might occur are skin rashes, abdominal discomfort or general malaise. The participants were advised that should any unusual discomfort or symptoms appear after taking herbal granules, they should stop the medication, contact the investigator, and/or seek medical advice from their 
G.P., if condition persisted after medication had been discontinued. The identity of the granules could be determined immediately to review which granules had been given. In the event of an anaphylactic shock, she should seek medical help as soon as possible.

Some participants experienced an undesirable taste that was only temporary, and posed no long-term adverse effect on the participants. Herbal granules could be put into capsules, or could be taken with honey, jam or juice to reduce or minimize the undesirable taste. If a participant still could not tolerate the taste of the herbal granules, she was free to withdraw from the study.

Each participant was advised that if she was concerned or felt distressed about any part of this study, counselling services could be provided. 
$\begin{array}{llr}\text { 8.1 Overview } & 197\end{array}$

$\begin{array}{lll}\text { 8.2 Demographic Profiles } & 199\end{array}$

8.2.1 General Health Behaviours 200

8.2.1.1 Smoking and Drinking Frequency and 200

Amount

8.2.1.2 Happiness At Work and Family Life 201

8.2.2 Menstrual Experiences 202

8.2.3 PMS History 203

$\begin{array}{lll}\text { 8.3 Scores on all Measures at Baseline } & 206\end{array}$

8.3.1 Psychological Characteristics 208

8.3.2 Traditional Chinese Medicine Patterns 209

$\begin{array}{ll}\text { 8.3.3 Tongue Sizes and Pulse Symptoms } & 210\end{array}$ 


\subsection{Overview}

A total of 236 women responded to the advertisements and were considered for comprehensive and clinical assessment for two consecutive months. One hundred and fifty two women met the inclusion criteria 1-9 and were willing to join the two month assessment phase of the study. Eighty seven of these women either failed to return the mailed questionnaires or did not meet criterion 10 (see subdivision 7.2.2). Sixty-five women who remained following assessment, were randomly allocated to either Group I or II and started treatment, but 4 withdrew (6.2\%) during the treatment period (one due to pregnancy and the other three did not like the taste of herbs/placebo). A total of 61 women eventually completed the entire treatment course with 31 in Group I, and 30 in Group II. A flow chart shown in Figure 8.1 next page schematically illustrates the management procedures for subject allocation and treatment delivery of the study.

At entry, there were no significant differences $(p>.05)$ between Group I and Group II on the participants' demographic characteristics and all baseline data on clinical assessment variables indicated that the two groups of women were drawn from the same population of Premenstrual Syndrome (PMS) sufferers. 


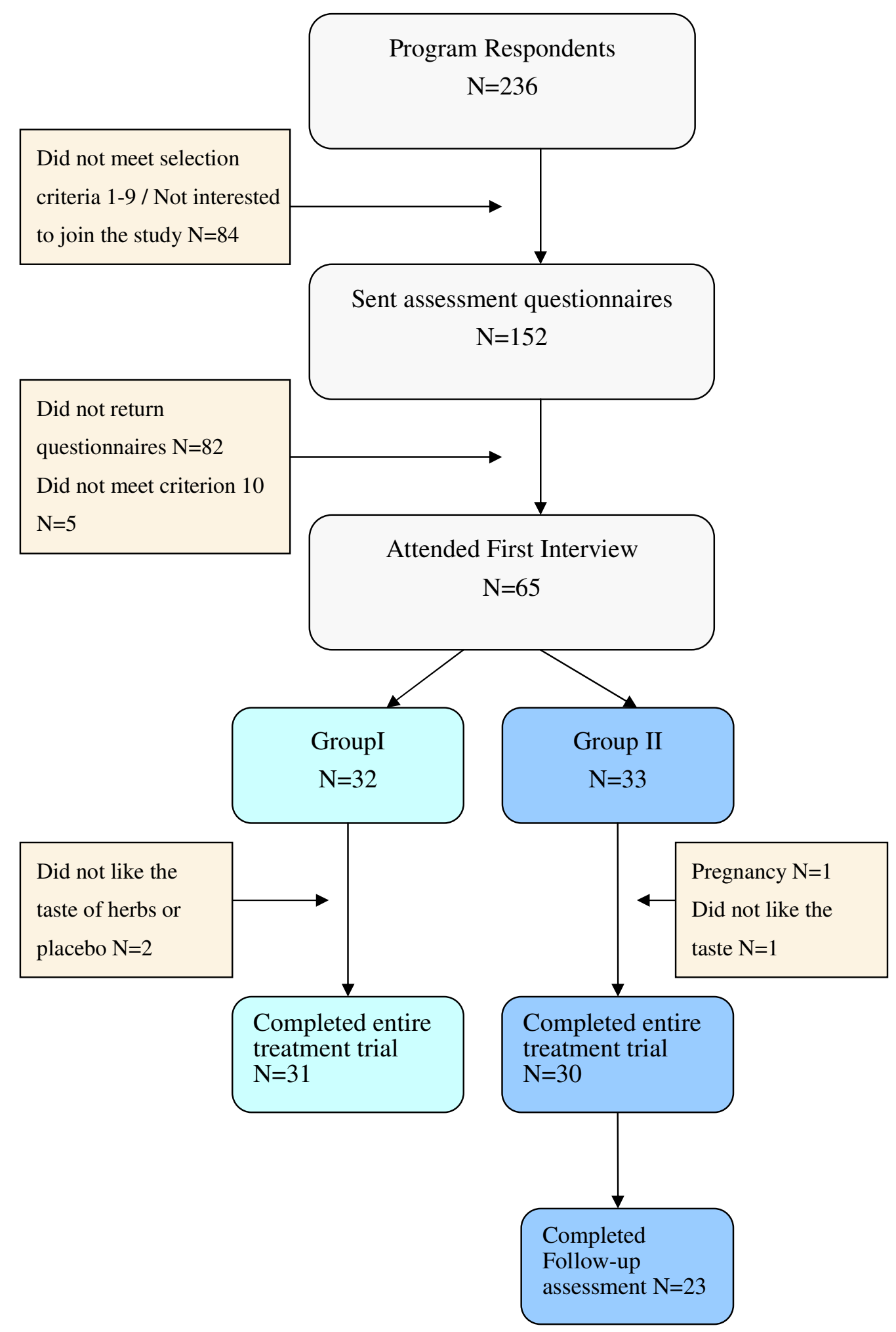

Figure 8.1 Summary diagram of participant flow 


\subsection{Demographic Profiles}

The demographic findings of all women $(n=65)$ who started treatment are outlined in Table 8.1 below:-

Table 8.1 : Demographic Profiles $(n=65)$

\begin{tabular}{|c|c|c|c|}
\hline Variables & Group I (n=32) & Group II (n=33) & All $(n=65)$ \\
\hline \multicolumn{4}{|l|}{ Age (yrs): } \\
\hline Mean (SD) & $32.4(6.13)$ & $31.1(6.07)$ & $31.8(6.08)$ \\
\hline Range & $19-40$ & $21-42$ & $19-42$ \\
\hline \multicolumn{4}{|l|}{ Marital status: n(\%) } \\
\hline Married & $13(40.6)$ & $14(42.4)$ & $27(41.5)$ \\
\hline Single & $19(59.4)$ & $19(57.6)$ & $38(58.5)$ \\
\hline \multicolumn{4}{|l|}{ Parity: n(\%) } \\
\hline Have child/children & $19(59.4)$ & $13(39.4)$ & $32(49)$ \\
\hline No child & $13(40.6)$ & $20(60.6)$ & $33(51)$ \\
\hline Median (Range) & $1(0-5)$ & $0(0-3)$ & $2(0-5)$ \\
\hline \multicolumn{4}{|c|}{ Highest education: $\mathbf{n}(\%)$} \\
\hline Secondary school & $8(25.0)$ & $7(21.2)$ & $15(23.1)$ \\
\hline Post-secondary & $5(15.6)$ & $9(27.3)$ & $14(21.5)$ \\
\hline University & $18(56.3)$ & $16(48.5)$ & $34(52.3)$ \\
\hline Others & 1( 3.1$)$ & $1(3.0)$ & $2(3.1)$ \\
\hline \multicolumn{4}{|l|}{ Occupation: n(\%) } \\
\hline Professional & $10(31.3)$ & $14(42.4)$ & 24(39.6) \\
\hline Semi-professional & $8(25.0)$ & $5(15.2)$ & $13(20)$ \\
\hline Small business & $2(6.3)$ & $1(3.0)$ & $3(4.6)$ \\
\hline Clerk or salesperson & $2(6.3)$ & $5(15.2)$ & $7(10.8)$ \\
\hline Unskilled & 0 & $3(9.1)$ & $3(4.6)$ \\
\hline Other & $10(31.3)$ & $5(15.2)$ & $15(23.1)$ \\
\hline \multicolumn{4}{|l|}{ Work: n(\%) } \\
\hline Full time & $12(37.5)$ & $15(45.5)$ & $27(41.5)$ \\
\hline Part time & $13(40.6)$ & $10(30.3)$ & $23(35.5)$ \\
\hline Unemployed & $1(3.1)$ & 0 & $1(1.5)$ \\
\hline Homemaker & 0 & $3(9.1)$ & $3(4.6)$ \\
\hline Student & $5(15.6)$ & $5(15.2)$ & $10(15.4)$ \\
\hline Other & $1(3.1)$ & 0 & $1(1.5)$ \\
\hline \multicolumn{4}{|l|}{ Ethnicity: n(\%) } \\
\hline Australasian & $9(28.1)$ & $11(33.3)$ & $20(30.8)$ \\
\hline British & $9(28.1)$ & $11(33.3)$ & $20(30.8)$ \\
\hline European & $12(37.5)$ & $8(24.2)$ & $20(30.8)$ \\
\hline Asian & $2(6.3)$ & $3(9.1)$ & $5(7.7)$ \\
\hline
\end{tabular}


The sixty-five women who started treatment had a mean age of 31.8 years with a range from 19-42 years. Twenty-seven (41.5\%) of them were married, and $38(58.5 \%)$ remained single. More than half $(51 \%)$ had no children. The majority $(52.31 \%)$ were university graduate, professionals, and were working full time. Of the 65 respondents, over $60 \%$ were from English speaking countries nearly a third (37.5\%) were European and a minority (6.3\%) were Asian.

\subsubsection{General Health Behaviours}

General health behaviours were assessed as substance use (nicotine, alcohol). Self reported satisfaction with family life and work was also assessed.

\subsubsection{Smoking and Drinking Frequency and Amount}

The majority (86.2\%) were non-smokers, and more than half of the sample (56.9\%) did not consume alcohol everyday whilst 11 did not drink at all, and no one reported having more than 3 glasses in a day. However, $24.6 \%$ women consumed 1-2 glass of alcoholic drinks per day (see Table 8.2). 
Table 8.2 Cigarette and alcohol consumption $(n=65)$

\begin{tabular}{lrrr}
\hline \multirow{2}{*}{\multicolumn{1}{c}{ Variables }} & \multicolumn{3}{c}{ Results } \\
\cline { 2 - 4 } & Group I & Group II & All \\
& $\mathrm{N}=32$ & $\mathrm{~N}=33$ & $\mathrm{~N}=65$ \\
\hline Cigarettes per day: n(\%) & & & \\
None & $26(81.3)$ & $30(90.9)$ & $56(86.2)$ \\
$1-10$ & $5(15.6)$ & $1(3.0)$ & $6(9.2)$ \\
$11-20$ & 0 & $2(6.1)$ & $2(3.1)$ \\
More than 20 & $1(3.1)$ & 0 & $1(1.5)$ \\
Alcoholic drinks per day (standard & & & \\
glasses): n(\%) & & & \\
None & $5(15.6)$ & $6(18.2)$ & $11(16.9)$ \\
Less than one (not everyday) & $18(56.3)$ & $19(57.6)$ & $37(56.9)$ \\
$1-2$ & $9(28.1)$ & $7(21.2)$ & $16(24.6)$ \\
$2-3$ & 0 & $1(3.0)$ & $1(1.5)$ \\
\hline
\end{tabular}

\subsubsection{Happiness At Work and Family Life}

Over $80 \%$ of the total sample reported they were happy at work and their family life as happy (Participants were only required to give a “yes' or 'no' answer). Data are shown in Table 8.3.

Table 8.3 Happiness at work and family life

\begin{tabular}{lrrc}
\hline \multirow{2}{*}{\multicolumn{1}{c}{ Variables }} & \multicolumn{3}{c}{ Result } \\
\cline { 2 - 4 } & Group I & Group II & All \\
& $\mathrm{N}=32$ & $\mathrm{~N}=33$ & $\mathrm{~N}=65$ \\
\hline Happy family life $\mathbf{n = 6 5 :} \mathbf{n}(\boldsymbol{\%})$ & & & \\
No & $7(21.9)$ & $2(6.1)$ & $9(13.8)$ \\
Yes & $25(78.1)$ & $31(93.9)$ & $56(86.2)$ \\
Happy at work $\mathbf{n = 6 5 : ~} \mathbf{n}(\%)$ & & & \\
No & $8(25.0)$ & $3(9.1)$ & $11(16.9)$ \\
Yes & $24(75.0)$ & $30(90.9)$ & $54(83.1)$ \\
\hline
\end{tabular}




\subsubsection{Menstrual Experiences}

The median age of menarche was 13 years and most (84.6\%) women experienced their first menstruation between 11 and 14 years of age. One case indicated delayed puberty as menarche did not occur until 19 years of age but her menstrual cycle was within the normal range, every 27 days with 5 days menstrual bleed. The length of the total menstrual cycle and days of menstruation were within the normal range with a median of 28 days (range 24-35days) and the median of bleeding days was 5 days (range 2-8). Menstrual History Profiles are provided in Table 8.4.

Table 8.4 Menstrual history $(n=65)$

\begin{tabular}{lrrr}
\hline \multicolumn{1}{c}{ Variables } & \multicolumn{3}{c}{ Results } \\
\cline { 2 - 4 } & Group I & \multicolumn{1}{c}{ Group II } & All \\
& $\mathrm{N}=32$ & $\mathrm{~N}=33$ & $\mathrm{~N}=65$ \\
\hline Menarche (age): & 13 & 13 & 13 \\
Median & $8(11-19)$ & $7(9-16)$ & $10(9-19)$ \\
Range & & & \\
Length of cycle (days): & 28 & 28 & 28 \\
Median & $10(25-35)$ & $9(24-33)$ & $11(24-35)$ \\
Range & & & \\
Length of menses (days) & 5 & 5 & 5 \\
Median & $6(2-8)$ & $4(3-7)$ & $6(2-8)$ \\
Range & & & \\
\hline
\end{tabular}


Self reported subjective menstrual characteristics before treatment are shown in Table 8.5 . The majority $(87.7 \%$ ) of these women usually or always had regular menstruation. More than half (53.8\%) reported a "normal" amount of bleeding, and the colour was mainly bright red and dark red (80\%). Most of them reported a "normal" thickness of bleeding and clots in the menses. $43.1 \%$ reported large clots and $55.4 \%$ reported small clots in their menses.

\subsubsection{PMS History}

At the first interview, the women were asked to describe their premenstrual experiences in terms of self-reported onset, severity and symptom relief. A median of 10 years (range 1.5-25) of PMS experience was reported.

Twenty-seven women (41.6\%) reported serious or severe adverse effects on their daily life, and 33 women $(50.8 \%)$ reported moderate adverse effects. The onset of symptoms ranged from 1 to 11 days with a median of 7 days, and the disappearance of symptoms occurred from the day before menses to the end of menstruation. The total days of PMS symptom experience ranged from 2 to 12 days with a median of 9 days. Thirty-seven of the women (56.9\%) had sisters (23), mothers (15), or daughters (2) who also suffered from PMS. Findings are shown in Table 8.6. 
Table 8.5 Menstrual characteristics $n(\%)$

\begin{tabular}{|c|c|c|c|}
\hline \multirow{3}{*}{ Variables } & \multicolumn{3}{|c|}{ Results } \\
\hline & Group I & Group II & All \\
\hline & $\mathrm{N}=32$ & $\mathrm{~N}=33$ & $\mathrm{~N}=65$ \\
\hline \multicolumn{4}{|l|}{ Regularity: n(\%) } \\
\hline Always & $10(31.3)$ & $13(39.4)$ & $23(35.4)$ \\
\hline Usually & $18(56.3)$ & $16(48.5)$ & $34(52.3)$ \\
\hline Occasionally & $4(12.5)$ & $4(12.1)$ & $8(12.3)$ \\
\hline \multicolumn{4}{|l|}{ Perceived Blood Loss: $n(\%)$} \\
\hline Very heavy & $3(9.4)$ & $1(3.0)$ & $4(6.2)$ \\
\hline Heavy & $16(50.0)$ & $10(30.3)$ & $19(29.2)$ \\
\hline Normal & $9(28.1)$ & $19(57.6)$ & $35(53.8)$ \\
\hline Scanty & $3(9.4)$ & $3(9.1)$ & $6(9.2)$ \\
\hline Missing & $1(3.1)$ & 0 & $1(1.5)$ \\
\hline \multicolumn{4}{|l|}{ Colour: n(\%) } \\
\hline Red & $4(12.5)$ & $6(18.2)$ & $10(15.4)$ \\
\hline Bright red & $13(40.6)$ & $12(36.4)$ & $25(38.5)$ \\
\hline Dark red & $13(40.6)$ & $14(42.4)$ & $27(41.5)$ \\
\hline Missing & $2(6.3)$ & $1(3.0)$ & $3(4.6)$ \\
\hline \multicolumn{4}{|l|}{ Nature: $\mathbf{n}(\%)$} \\
\hline Thin & $4(12.5)$ & $1(3.0)$ & $5(7.7)$ \\
\hline Normal & $17(53.1)$ & $25(75.8)$ & $42(64.6)$ \\
\hline Thick & $10(31.3)$ & $7(21.2)$ & $17(26.2)$ \\
\hline Missing & $1(3.1)$ & 0 & $1(1.5)$ \\
\hline \multicolumn{4}{|l|}{ Clots: $\mathbf{n}(\%)$} \\
\hline Large pieces & $16(50.0)$ & $12(36.4)$ & $28(43.1)$ \\
\hline Small pieces & $15(46.9)$ & $21(63.6)$ & $36(55.4)$ \\
\hline No & $1(3.1)$ & 0 & $1(1.5)$ \\
\hline
\end{tabular}


Table 8.6 PMS experiences

\begin{tabular}{|c|c|c|c|}
\hline \multirow{3}{*}{ Variables } & \multicolumn{3}{|c|}{ Results } \\
\hline & Group I & Group II & All \\
\hline & $\mathrm{N}=32$ & $\mathrm{~N}=33$ & $\mathrm{~N}=65$ \\
\hline \multicolumn{4}{|l|}{ Interferes with daily life: $n(\%)$} \\
\hline Incapacitating & $1(3.1)$ & $1(3.0)$ & $2(3.1)$ \\
\hline Severe & $15(46.9)$ & $10(30.3)$ & $25(38.5)$ \\
\hline Moderate & $14(43.8)$ & $19(57.6)$ & $33(50.8)$ \\
\hline Slight & $2(6.3)$ & $3(9.1)$ & $5(7.7)$ \\
\hline \multicolumn{4}{|l|}{ Symptom onset (days before } \\
\hline \multicolumn{4}{|l|}{ menses): } \\
\hline Median & 8 & 7 & 7 \\
\hline range & $9(2-11)$ & $9(1-10)$ & $10(1-11)$ \\
\hline \multicolumn{4}{|l|}{ Symptom relief (days): $\mathbf{n}(\%)$} \\
\hline day before menses & $7(21.9)$ & $7(21.2)$ & $13(20)$ \\
\hline By day 1 & $5(15.6)$ & $6(18.2)$ & $11(16.9)$ \\
\hline By day 2 & $10(31.3)$ & $9(27.3)$ & $19(29.2)$ \\
\hline By day 3 & $2(6.3)$ & $6(18.2)$ & $8(12.3)$ \\
\hline By day 4 & $6(18.8)$ & $3(9.1)$ & $9(13.8)$ \\
\hline After day 4 & $2(6.3)$ & $2(6.1)$ & $4(6.2)$ \\
\hline \multicolumn{4}{|l|}{ Total days of symptom } \\
\hline \multicolumn{4}{|l|}{ experience: } \\
\hline Median & 9.5 & 9 & 9 \\
\hline Range & $12(2-14)$ & $10(3-13)$ & $12(2-14)$ \\
\hline \multicolumn{4}{|l|}{ Years of PMS experience : } \\
\hline Mean (SD) & $10.75(6.72)$ & $9.92(6.55)$ & $10.3(6.59)$ \\
\hline Median & 10 & 10 & 10 \\
\hline Range & $23.5(1.5-25)$ & $23(2-25)$ & $23.5(1.5-25)$ \\
\hline \multicolumn{4}{|l|}{ Family PMS history: n(\%) } \\
\hline No & $12(37.5)$ & $16(48.5)$ & $28(43.1)$ \\
\hline Yes & $20(62.5)$ & $17(51.5)$ & $37(56.9)$ \\
\hline Mother & $11(34.4)$ & $12(36.4)$ & $23(35.4)$ \\
\hline Sister & $8(25.0)$ & $7(21.2)$ & $15(23.1)$ \\
\hline Daughter & $1(3.1)$ & $1(3.0)$ & $2(3.1)$ \\
\hline
\end{tabular}




\subsection{Scores on all Measures at Baseline}

The occurrence and severity of the presenting symptoms of concern before treatment were assessed using the Moos' Menstrual Distress Questionnaire (MDQ) at Day 1 or 2 of the menstrual cycle. The most common symptoms reported at the first interview were "Negative Affect" items (Irritability, Mood swings, Anxiety, Depression, and Tension), followed by "Pain" and "Water Retention" items (Fatigue, Swelling, Painful or tender breasts, and Headache). The least reported symptoms were the "Control" and "Autonomic" items. There was no significant difference $(\mathrm{p}>.05)$ between Group I and Group II on all symptom clusters at first interview. Clearly, this total group reported highly different levels of symptoms compared to the compared to the published norms of Moos and colleagues (1985), on all except Arousal. Individual and cluster symptoms of concern reported before treatment at the first interview are shown in Table 8.7 and 8.8 .

Table 8.7 Comparisons of Group I and Group II on symptoms by categorical clusters Mean (SD)

\begin{tabular}{lccccccc}
\hline \multirow{2}{*}{ Clusters } & Group I & Group II & \multicolumn{2}{c}{ Sig I/II } & & All & Norm(SD) \\
& $\mathrm{n}=32$ & $\mathrm{n}=33$ & $\mathrm{~F}$ & $\mathrm{P}$ & $\mathrm{n}=65$ & $*$ \\
\hline Autonomic reaction & $2.78(2.76)$ & $2.52(2.31)$ & .179 & .674 & $\mathbf{2 . 6 5}(2.52)$ & $0.69(0.90)$ \\
Behavior change & $8.28(5.01)$ & $6.79(3.22)$ & 2.056 & .157 & $\mathbf{7 . 5 2}(4.23)$ & $2.12(1.63)$ \\
Water retention & $7.25(2.96)$ & $7.64(3.82)$ & .207 & .651 & $\mathbf{7 . 4 5}(3.41)$ & $2.21(1.52)$ \\
Concentration & $10.06(6.38)$ & $8.64(4.69)$ & 1.060 & .307 & $\mathbf{9 . 3 4}(5.59)$ & $3.05(2.32)$ \\
Negative affect & $17.22(6.35)$ & $15.91(5.81)$ & .754 & .388 & $\mathbf{1 6 . 5 5}(6.07)$ & $5.21(3.66)$ \\
Pain & $10.97(5.06)$ & $9.33(3.81)$ & 2.176 & .145 & $\mathbf{1 0 . 1 4}(4.51)$ & $3.41(2.40)$ \\
Control & $3.00(3.91)$ & $2.73(3.11)$ & .097 & .756 & $\mathbf{2 . 8 6}(3.50)$ & $0.95(1.24)$ \\
Arousal & $4.06(3.63)$ & $4.36(3.07)$ & .131 & .719 & $4.22(3.33)$ & $4.33(2.03)$ \\
\hline
\end{tabular}


Table 8.8 Most common presenting symptoms $(\mathrm{n}=65)$

\begin{tabular}{lc}
\hline Present symptoms & $\mathrm{n}(\%)$ \\
\hline Irritability & $63(96.9)$ \\
Mood swings & $63(96.9)$ \\
Fatigue & $62(95.4)$ \\
Anxiety & $59(90.8)$ \\
Depression & $58(89.2)$ \\
Tension & $58(89.2)$ \\
Swelling & $57(87.7)$ \\
Change in eating habits & $56(86.2)$ \\
Difficulty concentrating & $55(84.6)$ \\
Decreased efficiency & $55(84.6)$ \\
Restlessness & $55(84.6)$ \\
Poor school or work performance & $54(83.1)$ \\
Painful or tender breasts & $54(83.1)$ \\
Headache & $54(83.1)$ \\
Forgetfulness & $53(81.5)$ \\
Avoid social activities & $52(80.0)$ \\
Crying & $52(80.0)$ \\
Wein blemish or disorder & $51(78.5)$ \\
Geneliness & $51(78.5)$ \\
\hline
\end{tabular}




\subsubsection{Psychological Characteristics}

The results of measures of anxiety (ANX), anger (ANG), depression (BDI) and self reported stress (PSS) obtained at the first assessment interview revealed these women were mildly to moderately depressed (range 10 to 18). The most frequent presenting reports on the BDI were Fatiguability, Sadness and Work difficulty. These women also scored higher than the norm means (18.64 and 13.71) for working female adults on State-anxiety and State-anger. Although the mean stress score (28.11) was slightly higher than the norm mean (PSS, 28.11 vs. 25.6) for females in the community, this was within one standard deviation of the norm (see Table 8.9).

Table 8.9: Psychological Characteristics $(n=65)$

\begin{tabular}{lccc}
\hline Measure & Mean & SD & $\begin{array}{l}\text { Norm* } \\
\text { M(SD) }\end{array}$ \\
\hline $\begin{array}{l}\text { STPI: } \\
\text { State-Anxiety }\end{array}$ & 25.88 & 6.05 & $18.64(6.84)$ \\
State-Anger & 19.75 & 6.30 & $13.71(5.72)$ \\
& & & \\
PSS: & & & \\
Perceived stress & 28.11 & 7.67 & $25.6(8.24)$ \\
& & & \\
BDI: & 11.25 & 5.35 & $4.73(4.73)$ \\
Total scores & & & \\
*STPI State-Anxiety and State-Anger norms( Spielberger 1979) & \\
*PSS norm (Cohen, Kamarck \& Mermelstein, 1983) & *BDI norm (Beck \& Beck 1972)
\end{tabular}




\subsubsection{Traditional Chinese Medicine Patterns}

The Pattern Identification method of traditional Chinese medicine (TCM) was revealed that Pattern H (32.3\%) and Pattern A (29.2\%) were the two dominant configurations, with Pattern CA and Pattern D accounting for $18.5 \%$ and $20 \%$ respectively. The distribution of TCM patterns is shown in Figure 8.2 and Table 8.10.

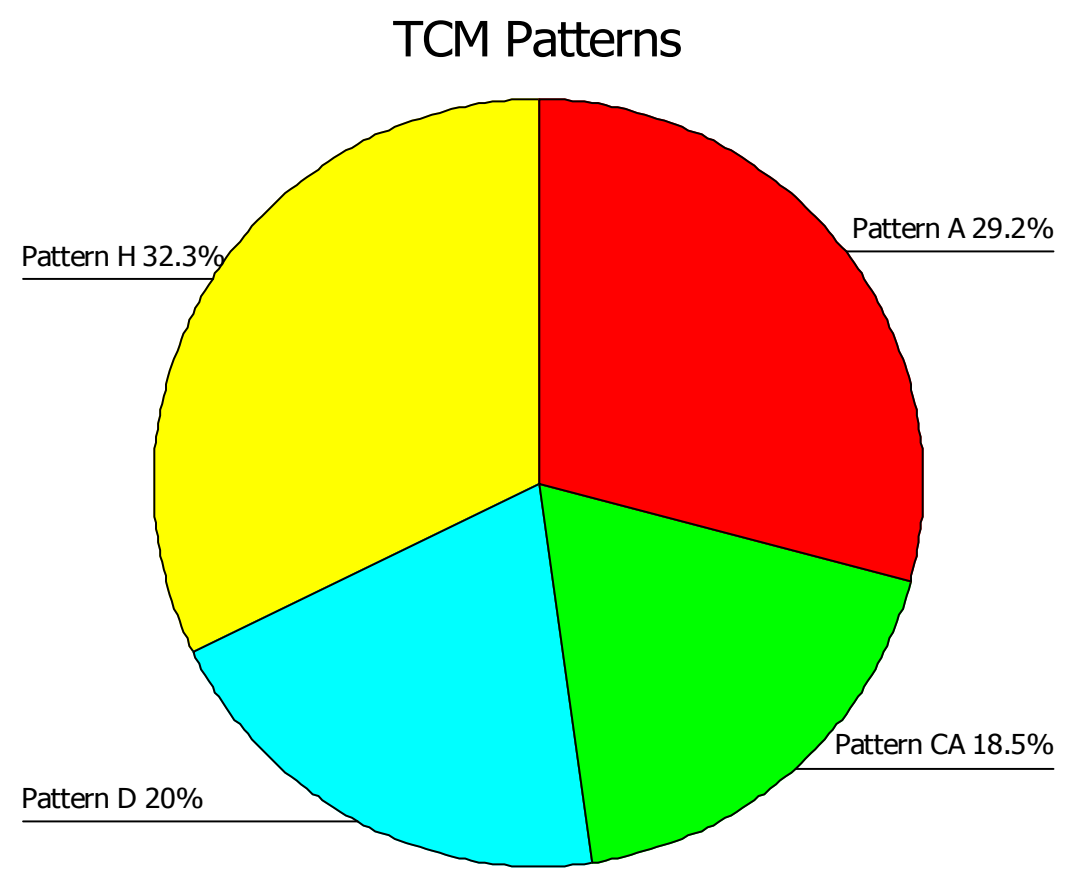

Figure 8.2 Distribution of TCM patterns 
Table 8.10 The distribution of Chinese patterns in groups $\mathrm{n}(\%)$

\begin{tabular}{llrrr}
\hline Patterns & Chief Symptoms & $\begin{array}{r}\text { Group I } \\
(\mathrm{n}=32)\end{array}$ & $\begin{array}{r}\text { Group II } \\
(\mathrm{n}=33)\end{array}$ & $\begin{array}{c}\text { All } \\
(\mathrm{n}=65)\end{array}$ \\
\hline Pattern A & Anxiety, irritability, nervous & $10(31.3)$ & $9(27.3)$ & $19(29.2)$ \\
(Liver Qi Stagnation) & tension, mood swing, breast & & & \\
& tenderness, hypochondrian & & & \\
& pain, lower abdominal pain & & & \\
Pattern CA & Head ache, dizziness, & $6(18.8)$ & $6(18.2)$ & 12(18.5) \\
(Liver Qi Stagnation, & tinnitus, oral ulcers, acne, & & & \\
Liver and Kidney Yin & red eyes, low grade fever, & & & \\
Deficiency) & increase appetite, cravings, & & & \\
& agitated, & & & \\
Pattern D & Fluid retention, swollen of & $5(15.6)$ & $8(24.2)$ & $13(20)$ \\
(Liver Qi Stagnation, & limbs, loose stool or & & & \\
Heart and Spleen & diarrhoea, fatigue, & & & \\
Deficiency) & abdominal bloating, reduce & & & \\
& appetite, breast distension, & & & \\
hypersomnia, weight gain & & & \\
Pattern H & Depression, crying, & $11(34.4)$ & $10(30.3)$ & 21(32.3) \\
(Liver Qi Stagnation, & forgetfulness, insomnia & & & \\
Liver and Spleen & (disturbed sleep), palpitation, & & & \\
Deficiency causing & reduce appetite, lethargy, & & & \\
fluid retention) & abdominal bloating & & & \\
\hline
\end{tabular}

\subsubsection{Tongue Sizes and Pulse Symptoms}

Examination of tongue sizes and pulse symptoms revealed that $45(69.2 \%)$ had a pink tongue, $27(41.5 \%)$ had teeth marks, 17 (26.2\%) showed some swelling, $21(32.3 \%)$ had vertical cracks and $28(43.1 \%)$ showed purplish areas (stagnation) on their tongues. The results also revealed that $44(67.7 \%)$ women 
had a thin tongue coating, $50(76.9 \%)$ had a white tongue coating, and 15 (23.1\%) had a yellowish tongue coating. All the signs of tongue coatings and bodies recorded in the first interview are shown in Table 8.11.

Table 8.11 Tongue signs recorded at the first interview

\begin{tabular}{llllllll}
\hline \multirow{2}{*}{ Tongue } & & \multicolumn{2}{l}{ Group I } & \multicolumn{2}{l}{ Group II } & \multicolumn{2}{l}{ All(n=65) } \\
\cline { 3 - 8 } Coating: & & $\mathrm{N}$ & $\%$ & $\mathrm{~N}$ & $\%$ & $\mathrm{~N}$ & $\%$ \\
Thickness & Moderate & 9 & 28.1 & 10 & 30.3 & $\mathbf{1 9}$ & $\mathbf{2 9 . 2}$ \\
& Thin & 22 & 68.8 & 22 & 66.7 & $\mathbf{4 4}$ & $\mathbf{6 7 . 7}$ \\
& Thick & 1 & 3.0 & 1 & 3.0 & $\mathbf{2}$ & $\mathbf{3 . 1}$ \\
Colour & White & 23 & 71.9 & 27 & 81.8 & $\mathbf{5 0}$ & $\mathbf{7 6 . 9}$ \\
& Yellow & 9 & 28.1 & 6 & 18.2 & $\mathbf{1 5}$ & $\mathbf{2 3 . 1}$ \\
Proper & Wet/slippery & 4 & 12.5 & 2 & 6.1 & $\mathbf{6}$ & $\mathbf{9 . 2}$ \\
& Dry & 1 & 3.0 & 0 & 0 & $\mathbf{1}$ & $\mathbf{1 . 5}$ \\
& Peeled/map & 4 & 12.5 & 1 & 3.0 & $\mathbf{5}$ & $\mathbf{7 . 7}$ \\
Body: & & & & & & & \\
Colour & Pink & 23 & 71.9 & 22 & 66.7 & $\mathbf{4 5}$ & $\mathbf{6 9 . 2}$ \\
& Pale & 4 & 12.5 & 4 & 12.1 & $\mathbf{8}$ & $\mathbf{1 2 . 3}$ \\
& Red & 4 & 12.5 & 6 & 18.2 & $\mathbf{1 0}$ & $\mathbf{1 5 . 4}$ \\
& Dark red & 1 & 3.0 & 1 & 3.0 & $\mathbf{2}$ & $\mathbf{3 . 1}$ \\
& Red Side & 4 & 12.5 & 7 & 21.2 & $\mathbf{1 1}$ & $\mathbf{1 6 . 9}$ \\
& Red Tip & 5 & 15.6 & 6 & 18.2 & $\mathbf{1 1}$ & $\mathbf{1 6 . 9}$ \\
& Teeth mark & 12 & 37.5 & 15 & 45.5 & $\mathbf{2 7}$ & $\mathbf{4 1 . 5}$ \\
& Swollen & 7 & 21.9 & 10 & 30.3 & $\mathbf{1 7}$ & $\mathbf{2 6 . 2}$ \\
& Thin \& small & 3 & 9.4 & 0 & 0 & $\mathbf{3}$ & $\mathbf{4 . 6}$ \\
& Cracks (Vertical) & 12 & 37.5 & 9 & 27.3 & $\mathbf{2 1}$ & $\mathbf{3 2 . 3}$ \\
& Cracks & 1 & 3.0 & 0 & 0 & $\mathbf{1}$ & $\mathbf{1 . 5}$ \\
& (Transverse) & & & & & & \\
& Purplish area & 15 & 46.9 & 13 & 39.4 & $\mathbf{2 8}$ & $\mathbf{4 3 . 1}$ \\
\hline \multirow{3}{*}{ Appearance } & & & & & & &
\end{tabular}


The most frequently presented pulses at the first interview were the wiry, thin and slippery pulses. Sixty two $(95.4 \%)$ of them had a wiry pulse, $48(73.1 \%)$ had a thin pulse and $45(69.2 \%)$ had a slippery pulse. All pulses recorded at the first interview are shown in Table 8.12.

Table 8.12 Pulses recorded at the first interview

\begin{tabular}{lllllll}
\hline & \multicolumn{2}{l}{ Group I $(\mathrm{n}=32)$} & \multicolumn{2}{l}{ Group II $(\mathrm{n}=33)$} & \multicolumn{2}{l}{ All(n=65) } \\
\cline { 2 - 7 } Pulses & $\mathrm{N}$ & $\%$ & $\mathrm{~N}$ & $\%$ & $\mathrm{~N}$ & $\%$ \\
\hline Wiry & 31 & 96.9 & 31 & 93.9 & $\mathbf{6 2}$ & $\mathbf{9 5 . 4}$ \\
Thin & 21 & 65.6 & 27 & 81.8 & $\mathbf{4 8}$ & $\mathbf{7 3 . 1}$ \\
Slippery & 25 & 28.1 & 20 & 60.6 & $\mathbf{4 5}$ & $\mathbf{6 9 . 2}$ \\
Choppy & 10 & 31.3 & 9 & 27.3 & $\mathbf{1 9}$ & $\mathbf{2 9 . 2}$ \\
Rapid & 7 & 21.9 & 1 & 3.0 & $\mathbf{8}$ & $\mathbf{1 2 . 3}$ \\
Weak & 1 & 3.1 & 3 & 9.1 & $\mathbf{4}$ & $\mathbf{6 . 2}$ \\
Scattered & 0 & 0 & 1 & 3.0 & $\mathbf{1}$ & $\mathbf{1 . 5}$ \\
\hline
\end{tabular}



Effect between Group I and Group II

9.1.2 Comparison of Herbal Treatment and Placebo Treatment Effects within Subjects of Group II

9.1.3 Summary

9.2 Comparing Pretest (baseline) and Posttest Outcome during the

9.2.1 Comparing Pretest and Posttest Outcome on Herbal 225 Treatment in Group I

9.2.2 Comparing Pretest and Posttest Outcomes on Herbal 230 Treatment in Group II

$\begin{array}{lll}\text { 9.2.3 Summary } & 234\end{array}$

9.3 Follow-up of Premenstrual Physical and Psychological 235 Symptoms

$\begin{array}{lll}9.4 & \text { Clinical Significance } & 238\end{array}$

9.4.1 Clinical Significance at the End of Herbal Treatment in 238 Group I

9.4.2 Clinical Significance at the End of Herbal Treatment in 240

9.4.3 Clinical Significance at Follow-up 241

$\begin{array}{lll}\text { 9.4.4 Summary } & 242\end{array}$ 


\subsection{Comparing Outcome Scores between Herbs and Placebo Treatment}

To examine the differences between herbal and placebo treatment between the two groups and within subjects in Group II, repeated measures MANOVA (multivariate analysis of variance) were performed for multivariate analyses and/or repeated measures ANOVA (analysis of variance) were performed for univariate analyses on variables including physical (PHYS) and psychological (PSYC) symptoms, depression (BDI), anxiety (ANX), anger (ANG) and stress (PSS).

\subsubsection{Comparison of Herbal Treatment and Placebo Treatment Effect between Group I and Group II}

The treatment effects of Chinese herbs and placebo were investigated using repeated measures MANOVA on all clinical variables comparing scores at baseline and the first three months of treatment of Group I (herbal treatment) and Group II (placebo treatment). The results indicate that there were significant differences $(\mathrm{p}<.05)$ between the two groups in all measures except for PSS (perceived stress) favouring the herbal treatment.

Pairwise comparisons on all clinical assessments at baseline and the first three months of treatment of the two groups also show significant differences ( $\mathrm{p}$ $\leqq .001$ ) in the constructed composite scores of PHYS (Pain, Water Retention 
and Autonomic Reaction) and PSYC (Concentration, Behavioral Change and Negative Affect) of the MDQ categories and significant difference $(p<.05)$ in depression scores after two months of treatment again favouring the herbal treatment.

Treatment effects on physical symptoms, psychological symptoms, depression, anxiety, anger and perceived stress across time in both groups are shown in Figure 9.1. Scores of all variables showed a marked reduction from baseline to the end of the first treatment month. By the second month, a further reduction in scores of physical symptoms, psychological symptoms and depression was apparent in Group I. There were also very small shifts in anxiety and anger, and no change in perceived stress. During the same time period, Group II showed an increase in physical and psychological symptoms and depression, no change in anger, but a reduction in anxiety and perceived stress. At the end of the third month, there were further reductions in all measured variables in Group I although depression and perceived stress achieved only a minimal reduction over the second month. In Group II, the scores revealed an increase in psychological symptoms (PSYC), depression, anger and perceived stress, a slight reduction in physical symptoms (PHYS), and no change in anxiety. There were highly significant differences $(\mathrm{p}<.001)$ between baseline and the end of the third month in all clinical assessments except perceived stress which only showed a significant difference of $\mathrm{p}<.05$ in Group I. In Group II, there were also significant differences $(\mathrm{p}<.05)$ indicated in the composite physical and psychological symptoms, and anxiety between these two time periods. 


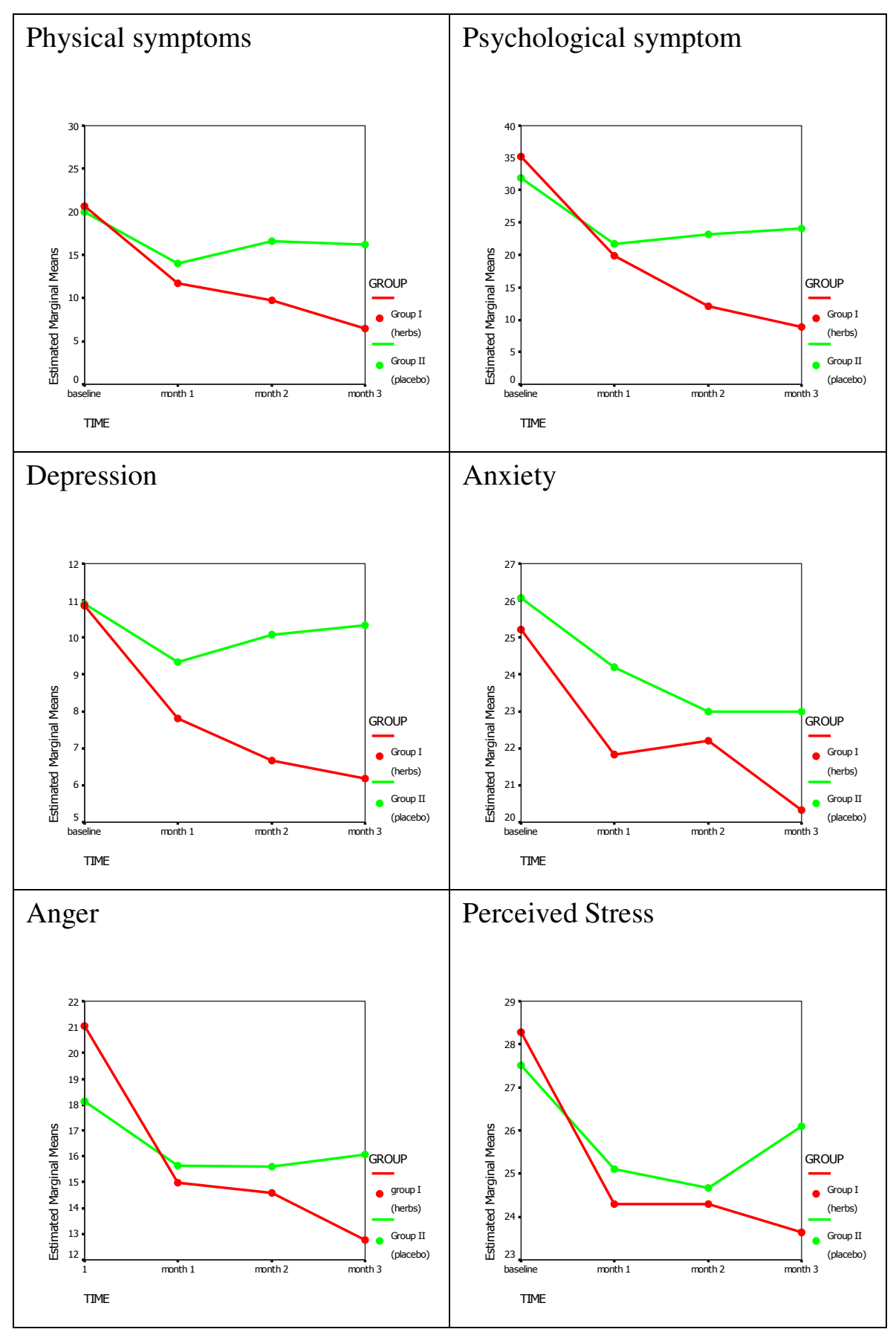

Figure 9.1: Effects of the baseline, three months herbal and placebo treatment on Physical symptoms, Psychological symptoms, Depression, Anxiety, Anger and Perceived Stress (Group I n=31, Group II n=30) 
However, the mean differences and percentages of reduction in all clinical assessment scores at the end of the third month in Group I were larger than the findings in Group II at the same time period. The largest reductions (68.9\% and $74.8 \%$ ) were shown in the composite physical and psychological symptoms of the MDQ in Group I. (see Table 9.1)

Table 9.1 Mean differences and percentages of reduction between baseline and month 3 of Group I (herb) and Group II (placebo)

\begin{tabular}{|c|c|c|c|c|c|c|}
\hline & \multicolumn{3}{|c|}{ Group I (n=31) } & \multicolumn{3}{|c|}{ Group II (n=30) } \\
\hline & $\begin{array}{l}\text { Mean } \\
\text { Differences }\end{array}$ & $\begin{array}{l}\% \text { of } \\
\text { reduction }\end{array}$ & Sig. & $\begin{array}{l}\text { Mean } \\
\text { Differences }\end{array}$ & $\begin{array}{l}\% \text { of } \\
\text { reduction }\end{array}$ & Sig. \\
\hline PHYS & 14.26 & 68.9 & .000 & 3.70 & 18.6 & .037 \\
\hline PSYC & 26.35 & 74.8 & .000 & 6.60 & 20.7 & .003 \\
\hline BDI & 4.68 & 43.1 & .000 & .57 & 5.2 & 1.000 \\
\hline ANX & 6.00 & 23.8 & .000 & 3.07 & 11.8 & .006 \\
\hline ANG & 8.26 & 39.3 & .000 & 2.07 & 7.9 & .058 \\
\hline PSS & 4.65 & 16.4 & .011 & 1.40 & 5.1 & 1.000 \\
\hline
\end{tabular}

In summary, there were highly significant differences $(\mathrm{p}<.01)$ after three months of treatment between Chinese herbs and placebo in physical and psychological symptoms, depression, anxiety and anger, but with no difference in perceived stress $(\mathrm{p}>.05)$ in these two groups of women. The results of all clinical assessments showed an initial marked reduction by the end of the first month of the experiment in both groups. With Group I (herbs), there were further reductions across month 2 and month 3 in all results except for perceived stress. Conversely, after the first month, the scores of Group II 
increased or worsened in all assessments except in anxiety which remained static after two months of treatment.

\subsubsection{Comparison of Herbal Treatment and Placebo Treatment Effects within Subjects of Group II}

The women in Group II received placebo for the first three months and herbal medication for the following three months. Data collected from Group II were used to compare the treatment effects between Chinese herbs and placebo. Data collected from Group I were not examined, since there was no washout period between the herbal and placebo treatment in the study, and carryover effect from herbal treatment might affect the results during placebo treatment.

In order to examine the differences in outcome between the herbal treatment and placebo treatment condition in the same group of subjects, MANOVA for repeated measures analyses were conducted on all variables including PHYS, PSYC, BDI, ANX, ANG and PSS at the baseline and for all six months of treatment. The findings indicate that there were significant differences across time phases [Wilks' Lambda $=.094, \mathrm{~F}(6,24)=38.95, \mathrm{p}<.001$ ], and the within-subjects interaction between measure and time [Greenhouse-Geisser F $(10.433,302.543)=22.121, \mathrm{p}<.001]$ was also significant. Outcomes of all clinical assessments at different time stages of Group II are shown in Figure 9.2. 


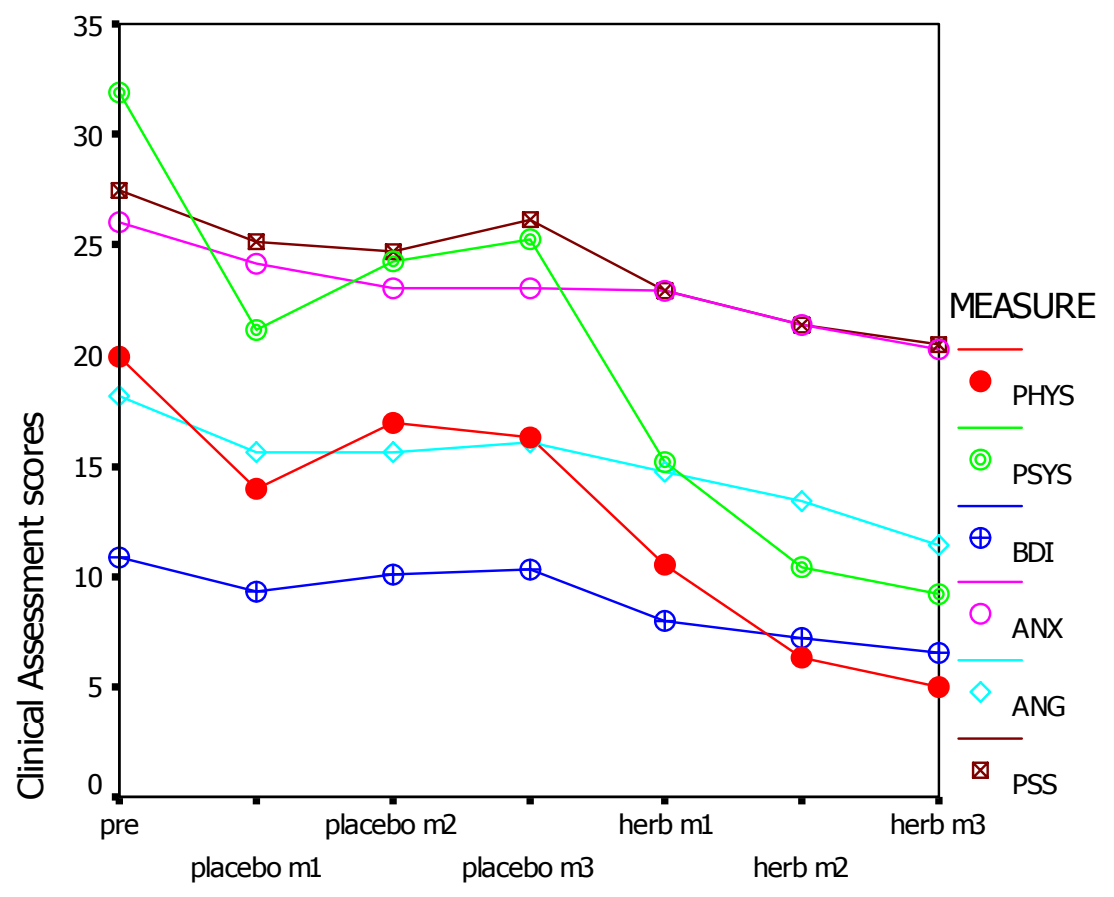

Figure 9.2 Treatment outcomes of Group II $(n=30)$

From the result of paired comparisons of time phases (Table 9.2), there were highly significant differences $(\mathrm{p}<.001)$ in scores between placebo month 3 and herbal treatment month 3 . There were also significant differences $(\mathrm{p}<.05)$ in scores between baseline and all other time phases, placebo month 3 and herbal treatment month 1, herbal treatment month 1 and month 2, and herbal treatment month 2 and month 3 . There were no significant differences in scores between placebo month 1 and month 2, or placebo month 2 and month 3 . 
Table 9.2 Significance of mean differences between time phases of Group

\begin{tabular}{|c|c|c|c|}
\hline \multicolumn{4}{|c|}{ II (n=30) } \\
\hline Time (I) & Time (J) & $\begin{array}{c}\text { Mean Differences } \\
\text { I-J }\end{array}$ & $P$ value \\
\hline pre & Placebo m1 & 4.18 & .000 \\
\hline pre & Placebo m2 & 3.30 & .003 \\
\hline pre & Placebo m3 & 2.90 & .001 \\
\hline pre & Herb m1 & 6.71 & .000 \\
\hline pre & Herb m2 & 9.06 & .000 \\
\hline pre & Herb m3 & 10.26 & .000 \\
\hline Placebo m1 & Placebo m2 & -.88 & 1.000 \\
\hline Placebo m2 & Placebo m3 & -.40 & 1.000 \\
\hline Placebo m3 & Herb m1 & 3.81 & .000 \\
\hline Herb m1 & Herb m2 & 2.36 & .001 \\
\hline Herb m2 & Herb m3 & 1.20 & .047 \\
\hline Placebo m3 & Herb m3 & 7.36 & .000 \\
\hline
\end{tabular}

Further analyses using ANOVA for repeated measures was carried out on each clinical assessment scale. The result shown in (Table 9.3) indicates that there were significant effects across time phases on all assessments in Group II.

Table 9.3 Time effects on all clinical assessments in Group II ( $\mathrm{n}=30)$

\begin{tabular}{lrrrr}
\hline Time Effect & \multicolumn{5}{c}{ Wilks' Lambda } \\
\hline & Value & F & Sig & Eta square \\
PHYS & .17 & 19.94 & .000 & .83 \\
PSYC & .11 & 31.32 & .000 & .89 \\
BDI & .25 & 11.90 & .000 & .75 \\
ANX & .34 & 7.85 & .000 & .66 \\
ANG & .27 & 10.74 & .000 & .73 \\
PSS & .41 & 5.75 & .001 & .59 \\
\hline
\end{tabular}


Pairwise comparisons of all clinical assessment scores between various time levels during both treatment application of placebo and herbs of Group II were undertaken.

The results demonstrated a reduction between baseline and the first month of placebo treatment in all scores (see Table 9.4). However, statistically significant differences $(\mathrm{p} \leqq .001)$ were only shown in the scores of the two composite physical and psychological variables (PHYS, PSYC) as measured on the MDQ. The scores in physical symptoms, psychological symptoms, depression, anger and perceived stress showed an increase or were static $(\mathrm{p}=$ 1.000) during the next two placebo treatment months, although anxiety scores showed a slight decrease in placebo month 2 , and then remained at the same level in placebo month 3 . There was no significant difference between baseline scores and placebo treatment scores at month 3 in physical symptoms, depression, anger and perceived stress, but significant differences $(\mathrm{p}<.05)$ were found in psychological symptoms and anxiety between these two time stages.

In the herbal treatment condition, there was marked reduction in the scores of physical symptoms and psychological symptoms at all measurement phases. Statistically significant differences $(\mathrm{p}<.05)$ were observed between placebo month 3 and herbal treatment month 1 (the third and fourth treatment month of the study) in most measured variables except anxiety and anger. There was also marked reductions $(\mathrm{p}<.005)$ in physical and psychological symptoms between 
herbal month 1 and 2 (the forth and fifth treatment month). Thereafter, reduction in scores continued although at a slower pace except in depression where there was a slight increase between herbal treatment month 2 and 3 (the fifth and sixth treatment month). There were highly significant differences ( $p$ $\leqq .001$ ) between scores comparing baseline with the end of herbal treatment in all assessments favouring the active substance. There were also significant differences between scores on most variables by the end of the placebo treatment and herbal treatment $(\mathrm{p}<.001)$ except in anxiety $(\mathrm{p}<.05)$. The results of the mean differences between the end of placebo treatment and the end of herbal treatment are highlighted in Table 9.4. 
Table 9.4 Mean differences of clinical assessment scores between baseline, placebo and herbal treatment months in Group II $(\mathrm{n}=30)$

\begin{tabular}{|c|c|c|c|c|}
\hline & Time (I) & Time $(\mathrm{J})$ & Mean Diff I-J & Sig. \\
\hline \multirow[t]{9}{*}{ PHYS } & Baseline & Placebo month 1 & 5.93 & .001 \\
\hline & Placebo month 1 & Placebo month 2 & -2.97 & .044 \\
\hline & Placebo month 2 & Placebo month 3 & .73 & 1.000 \\
\hline & Baseline & Placebo month 3 & 3.70 & .136 \\
\hline & Placebo month 3 & Herbal month 1 & 5.70 & .000 \\
\hline & Herbal month 1 & Herbal month 2 & 4.20 & .000 \\
\hline & Herbal month 2 & Herbal month 3 & 1.37 & .537 \\
\hline & Baseline & Herbal month 3 & 14.97 & .000 \\
\hline & Placebo month 3 & Herbal month 3 & 11.27 & .000 \\
\hline \multirow[t]{9}{*}{ PSYC } & Baseline & Placebo month 1 & 10.8 & .000 \\
\hline & Placebo month 1 & Placebo month 2 & -3.17 & 1.000 \\
\hline & Placebo month 2 & Placebo month 3 & -1.03 & 1.000 \\
\hline & Baseline & Placebo month 3 & 6.60 & .009 \\
\hline & Placebo month 3 & Herbal month 1 & 10.17 & .000 \\
\hline & Herbal month 1 & Herbal month 2 & 4.73 & .002 \\
\hline & Herbal month 2 & Herbal month 3 & 1.23 & 1.000 \\
\hline & Baseline & Herbal month 3 & 22.73 & .000 \\
\hline & Placebo month 3 & Herbal month 3 & 16.13 & .000 \\
\hline \multirow[t]{9}{*}{ BDI } & Baseline & Placebo month 1 & 1.57 & .667 \\
\hline & Placebo month 1 & Placebo month 2 & -7.33 & 1.000 \\
\hline & Placebo month 2 & Placebo month 3 & -2.67 & 1.000 \\
\hline & Baseline & Placebo month 3 & .57 & 1.000 \\
\hline & Placebo month 3 & Herbal month 1 & 2.37 & .001 \\
\hline & Herbal month 1 & Herbal month 2 & -.77 & 1.000 \\
\hline & Herbal month 2 & Herbal month 3 & -.67 & 1.000 \\
\hline & Baseline & Herbal month 3 & 4.37 & .000 \\
\hline & Placebo month 3 & Herbal month 3 & 3.80 & .000 \\
\hline \multirow[t]{9}{*}{ ANX } & Baseline & Placebo month 1 & 1.87 & .517 \\
\hline & Placebo month 1 & Placebo month 2 & 1.20 & 1.000 \\
\hline & Placebo month 2 & Placebo month 3 & .00 & 1.000 \\
\hline & Baseline & Placebo month 3 & 3.07 & .022 \\
\hline & Placebo month 3 & Herbal month 1 & .10 & 1.000 \\
\hline & Herbal month 1 & Herbal month 2 & 1.57 & .309 \\
\hline & Herbal month 2 & Herbal month 3 & 1.03 & 1.000 \\
\hline & Baseline & Herbal month 3 & 5.77 & .000 \\
\hline & Placebo month 3 & Herbal month 3 & 2.70 & .020 \\
\hline \multirow[t]{9}{*}{ ANG } & Baseline & Placebo month 1 & 2.50 & .248 \\
\hline & Placebo month 1 & Placebo month 2 & -.03 & 1.000 \\
\hline & Placebo month 2 & Placebo month 3 & -.47 & 1.000 \\
\hline & Baseline & Placebo month 3 & 2.07 & .203 \\
\hline & Placebo month 3 & Herbal month 1 & 1.30 & .362 \\
\hline & Herbal month 1 & Herbal month 2 & 1.37 & 1.000 \\
\hline & Herbal month 2 & Herbal month 3 & 1.97 & .198 \\
\hline & Baseline & Herbal month 3 & 6.70 & .000 \\
\hline & Placebo month 3 & Herbal month 3 & 4.63 & .000 \\
\hline \multirow[t]{9}{*}{ PSS } & Baseline & Placebo month 1 & 2.40 & .583 \\
\hline & Placebo month 1 & Placebo month 2 & .37 & 1.000 \\
\hline & Placebo month 2 & Placebo month 3 & -1.37 & 1.000 \\
\hline & Baseline & Placebo month 3 & 1.40 & 1.000 \\
\hline & Placebo month 3 & Herbal month 1 & 3.20 & .013 \\
\hline & Herbal month 1 & Herbal month 2 & 1.50 & .530 \\
\hline & Herbal month 2 & Herbal month 3 & .93 & 1.000 \\
\hline & Baseline & Herbal month 3 & 7.03 & .001 \\
\hline & Placebo month 3 & Herbal month 3 & 5.63 & .000 \\
\hline
\end{tabular}




\subsubsection{Summary}

There were highly significant differences $(p<.001)$ on scores between the end of the placebo and herbal treatment within all clinical assessment variables favouring the active herbal substance in women of Group II. In the univariate analysis, there were also highly significant differences $(\mathrm{p} \leqq .001)$ between scores by the end of the placebo and herbal treatments in most assessments except on anxiety $(\mathrm{p}<.05)$ again favouring the active herbal substance. There was no significant difference between baseline scores and end of placebo treatment scores in physical symptoms, depression, anger and perceived stress. Subsequently, there were highly significant differences $(\mathrm{p} \leqq .001)$ between scores at baseline and by the end of herbal treatment in all assessments favouring herbal treatment in this group of women.

From both comparisons between groups and within subjects, there were statistically significant differences in outcomes between herbal and placebo treatments between Group I and Group II and within subjects of Group II overtime.

\subsection{Comparing Pretest (baseline) and Posttest Outcome during the Herbal Treatment Period}

To examine the score differences between pre-treatment and the herbal treatment period at three months, repeated measures MANOVA were performed for multivariate analyses and/or repeated measures ANOVA were 
performed for univariate analyses on all assessment variables including PHYS, PSYC, BDI, ANX, ANG and PSS.

\subsubsection{Comparing Pretest and Posttest Outcome on Herbal Treatment in Group I}

The women in Group I received herbal treatment in the first three months of the study. Data collected the month before commencement of treatment and during the first three months of the study on these women were used for analysis.

The treatment effects of herbal medicine at different time phases were investigated using repeated measures MANOVA on all clinical variables comparing baseline and each of the first three months of the herbal treatment condition of Group I. The results reveal that there were significant differences $(p<.001)$ between these four time phases and measures by time interactions (Table 9.5).

Table 9.5 Time and Measurement Effects between baseline and three months herbal treatment in Group I $(n=31)$

\begin{tabular}{lcccc}
\hline Effect & \multicolumn{4}{c}{ Wilks' Lambda } \\
\hline \multirow{2}{*}{ Time } & Value & F & Sig & Eta square \\
Measure x Time & .118 & $69.47(3,28)$ & .000 & .882 \\
\hline
\end{tabular}


Further analysis, using repeated measures MANOVA, on each of the clinical assessments of Group I was carried out to reveal the differences in effect on all the symptoms. The results of the multivariate tests indicate that there were highly significant differences $(\mathrm{p}<.005)$ over the four time periods in all six clinical assessment scores. These results are shown in Table 9.6.

Table 9.6 Effects of herbal treatment on symptom reports in Group I

\begin{tabular}{lcccc}
\multicolumn{2}{c}{$(\mathrm{n}=31)$} & & & \\
\hline Variable & Wilk's $\Lambda$ & $\mathrm{F}(3,28)$ & Sig & Eta square \\
\hline PHYS & .17 & 46.30 & .000 & .83 \\
PSYC & .16 & 48.97 & .000 & .84 \\
BDI & .50 & 9.17 & .000 & .99 \\
ANX & .47 & 10.45 & .000 & .996 \\
ANG & .34 & 17.95 & .000 & .66 \\
PSS & .62 & 5.76 & .003 & .92 \\
\hline
\end{tabular}

The treatment outcomes of the clinical assessments which are shown in Figure 9.3 indicate that there was a marked reduction from the baseline through to the end of the first month of herbal treatment in all clinical assessment scores.

The constructed composite scores of physical and psychological symptoms of the MDQ (PHYS, PSYC) both showed marked reductions from baseline to the end of herbal treatment month 1 with a modest continued reduction to the end of month 3. All psychological assessment scores including depression (BDI), anxiety (ANX), anger (ANG) and perceived stress (PSS) also showed substantial reduction between baseline and the end of first herbal treatment 
month. The reduction in these psychological symptom scores showed very small shifts by the end of the second month of herbal treatment. After that, there was a further reduction at the end of the third month in all these scores except PSS.

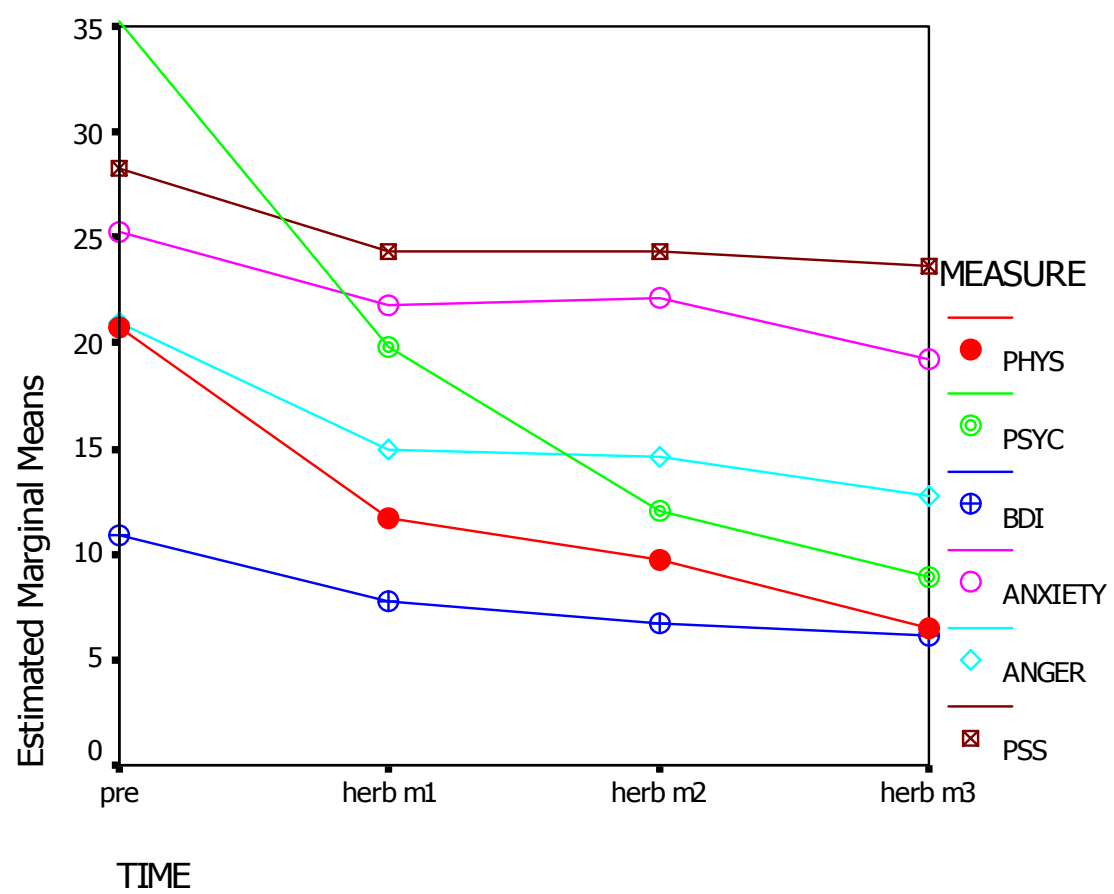

Figure 9.3 Treatment outcomes comparing baseline and three months of herbal treatment in Group I $(n=31)$

Pairwise comparisons of all clinical assessment scores between various time levels during treatment application of herbs of Group I were undertaken. The results are shown in Table 9.7. 
Table 9.7 Comparisons between baseline and three months of herbal treatment on Physical symptoms, Psychological symptoms, Depression, Anxiety, Anger and Perceived Stress in Group I

\begin{tabular}{|c|c|c|c|c|c|}
\hline & Time (I) & Time (J) & $\begin{array}{r}\text { Mean Diff } \\
\text { I-J } \\
\end{array}$ & Sig & $\begin{array}{l}\% \text { of } \\
\text { reduction }\end{array}$ \\
\hline \multirow[t]{4}{*}{ PHYS } & Baseline & Herbal month 1 & 8.97 & .000 & 43.3 \\
\hline & Herbal month 1 & Herbal month 2 & 2.03 & .415 & 17.3 \\
\hline & Herbal month 2 & Herbal month 3 & 3.26 & .001 & 33.6 \\
\hline & Baseline & Herbal month 3 & 14.26 & .000 & 68.9 \\
\hline \multirow[t]{4}{*}{ PSYC } & Baseline & Herbal month 1 & 15.39 & .000 & 43.7 \\
\hline & Herbal month 1 & Herbal month 2 & 7.84 & .001 & 39.5 \\
\hline & Herbal month 2 & Herbal month 3 & 3.13 & .027 & 26.1 \\
\hline & Baseline & Herbal month 3 & 26.36 & .000 & 74.8 \\
\hline \multirow[t]{4}{*}{ BDI } & Baseline & Herbal month 1 & 3.07 & .018 & 28.2 \\
\hline & Herbal month 1 & Herbal month 2 & 1.13 & .721 & 14.5 \\
\hline & Herbal month 2 & Herbal month 3 & .48 & 1.000 & 7.2 \\
\hline & Baseline & Herbal month 3 & 4.68 & .000 & 43.1 \\
\hline \multirow[t]{4}{*}{ ANX } & Baseline & Herbal month 1 & 3.88 & .012 & 15.4 \\
\hline & Herbal month 1 & Herbal month 2 & -.36 & 1.000 & -1.6 \\
\hline & Herbal month 2 & Herbal month 3 & 2.97 & .020 & 13.4 \\
\hline & Baseline & Herbal month 3 & 6.00 & .000 & 23.8 \\
\hline \multirow[t]{4}{*}{ ANG } & Baseline & Herbal month 1 & 6.07 & .000 & 28.9 \\
\hline & Herbal month 1 & Herbal month 2 & .387 & 1.000 & 2.6 \\
\hline & Herbal month 2 & Herbal month 3 & 1.81 & .038 & 12.4 \\
\hline & Baseline & Herbal month 3 & 8.26 & .000 & 39.3 \\
\hline \multirow[t]{4}{*}{ PSS } & Baseline & Herbal month 1 & 4.00 & .012 & 14.1 \\
\hline & Herbal month 1 & Herbal month 2 & .000 & 1.000 & 0 \\
\hline & Herbal month 2 & Herbal month 3 & .645 & 1.000 & 4.4 \\
\hline & Baseline & Herbal month 3 & 4.65 & .011 & 16.4 \\
\hline
\end{tabular}


There were significant reductions $(\mathrm{p}<.05)$ in all clinical assessment scores between baseline and the end of first herbal treatment month. By the end of the second month, there was no further significant reduction $(\mathrm{p}>.05)$ in all assessment scores except psychological symptoms of the MDQ. At the end of herbal treatment month 3 , significant reductions $(\mathrm{p}<.05)$ from month 2 were shown in scores of premenstrual physical and psychology symptoms of the MDQ, anxiety and anger, but no significant differences $(p>.05)$ in depression and perceived stress. However, there were highly significant reductions ( $\mathrm{p}$ $<.001$ ) in premenstrual physical and psychological symptoms, depression, anxiety and anger, and also a significant reduction $(\mathrm{p}<.05)$ in perceived stress between baseline and end of the third herbal treatment month. The largest reductions were shown in physical and psychological symptoms $(68.9 \%$ and $74.8 \%$ ) as measured on the MDQ.

In summary, these findings show that there were marked treatment effects on all clinical symptoms at the end of the herbal treatment in comparison to symptom reports before the commencement of treatment. The largest treatment effect was shown in the first month of herbal treatment when the percentage of reduction in symptom scores was most prominent. It then slowed down but was still obvious and continued in the direction of improvement. The herbal treatment was shown to be most effective for premenstrual physical and psychological symptoms, and also in reducing anxiety, anger and depression. Self reported stress also reduced over the same time period to a more modest extent. 


\subsubsection{Comparing Pretest and Posttest Outcomes on Herbal Treatment in Group II}

The women in Group II received placebo in the first three months of the study and herbal treatment from the fourth to sixth months. Data collected before commencement of treatment and during herbal treatment were examined to investigate the treatment effect of herbal medicine on PMS women. Since there was no washout period between the placebo and herbal treatment, data collected from the last month of the placebo treatment time period (i.e. the third month of the study) was also examined to reflect the latest symptom severity of the subject. Data were collected on PHYS, PSYC, BDI, ANX, ANG, and PSS at baseline, and in the third, fourth, fifth and sixth months of the study on these women and used for analysis using MANOVA for repeated measures. The results reveal significant differences $(\mathrm{p}<.001)$ between these time phases and measures by time interactions (Table 9.8).

Table 9.8 Time and Measures Effects and Measures by Time Interactions between baseline and three months herbal treatment in Group II

\begin{tabular}{lcccc}
\multicolumn{5}{c}{$(\mathrm{n}=30)$} \\
\hline Effect & Value & F & Sig & Eta square \\
& .113 & $50.82(4.26)$ & .000 & .89 \\
Time & .043 & $110.82(5,25)$ & .000 & .96 \\
Measures & .047 & $10.08(20,10)$ & .000 & .95 \\
Measures x Time & & &
\end{tabular}


Further analysis using repeated measures MANOVA on each of the clinical assessments in Group II was carried out to examine the differences in effect on all the symptoms. The results of the multivariate analyses indicate highly significant differences $(\mathrm{p}<.001)$ over the time periods in all six clinical assessment scores (PHYS, PSYC, BDI, ANX, ANG, PSS). These are shown in Table 9.9.

Table 9.9 Significant differences over the four time periods (the last month of placebo treatment and the three months of herbal treatment $)$ in Group II $(\mathrm{n}=30)$

\begin{tabular}{lcccc}
\hline Variable & Wilk's $\Lambda$ & $\mathrm{F}(4,26)$ & Sig & Eta square \\
\hline PHYS & .18 & 30.26 & .000 & .82 \\
PSYC & .13 & 43.57 & .000 & .87 \\
BDI & .26 & 18.22 & .000 & .74 \\
ANX & .36 & 11.64 & .000 & .64 \\
ANG & .31 & 14.51 & .000 & .69 \\
PSS & .46 & 7.70 & .000 & .54 \\
\hline
\end{tabular}

The treatment outcomes of these clinical assessments are shown in Figure 9.4 and indicate a reduction from the baseline and the last month of placebo treatment through to the end of the herbal treatment on all clinical assessment scores. Marked reductions occurred in all clinical assessment scores at the end of the first month of herbal treatment. The reduction then slowed but continued over the next two months 


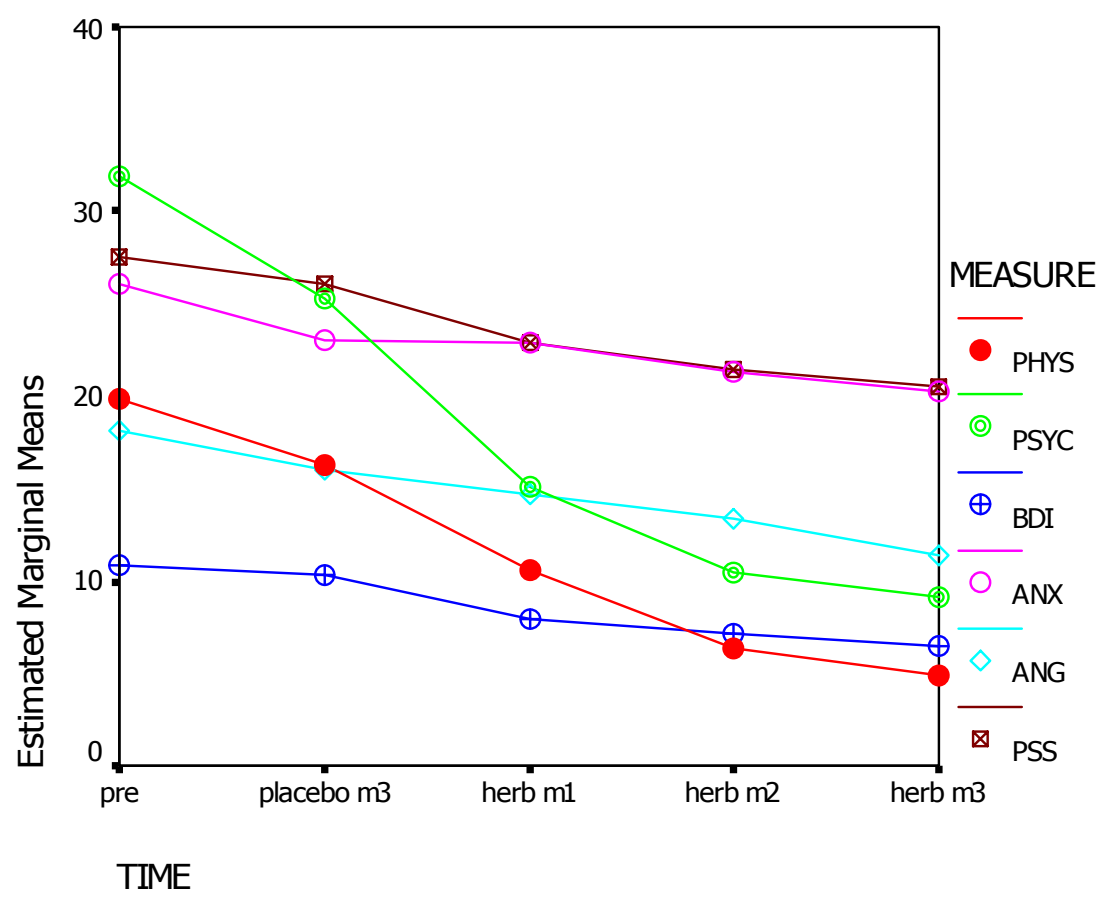

\section{Figure 9.4 Treatment outcomes of pre-treatment and three months herbal treatment in Group II $(n=30)$}

Pairwise comparisons between time levels during treatment application of herbs were explored on all assessment scores. The results shown in Table 9.10 indicate that comparisons between baseline and end of herbal treatment month 1 (month 4 of the study) were statistically significant $(\mathrm{p}<.01)$ in reducing scores on all measures. Between the end of herbal treatment month 1 and 2 (month 4 and 5 of the study), significant reductions ( $\mathrm{p} \leqq .001$ ) were demonstrated only in the composite physical (PHYS) and psychological (PSYC) symptoms. Between the end of second and third herbal treatment month (month 5 and 6 of the study), no significant differences $(p>.05)$ were 
Table 9.10 Comparisons between baseline and three months of herbal treatment period of Physical symptoms, Psychological symptoms, Depression, Anxiety, Anger and Perceived Stress in Group II $(\mathrm{n}=30)$

\begin{tabular}{|c|c|c|c|c|c|}
\hline Variable & Time (I) & Time (J) & $\begin{array}{r}\text { Mean Diff } \\
\text { I-J }\end{array}$ & Sig & $\begin{array}{l}\% \text { of } \\
\text { reduction }\end{array}$ \\
\hline \multirow[t]{5}{*}{ PHYS } & Baseline & Herb m1 & 9.40 & .000 & 47.2 \\
\hline & Herb m1 & Herb m2 & 4.20 & .000 & 39.9 \\
\hline & Herb m 2 & Herb m3 & 1.37 & .537 & 21.6 \\
\hline & Placebo $\mathrm{m} 3$ & Herb m3 & 11.27 & .000 & 69.9 \\
\hline & Baseline & Herb m3 & 14.97 & .000 & 72.3 \\
\hline \multirow[t]{5}{*}{ PSYC } & Baseline & Herb m1 & 16.77 & .000 & 52.6 \\
\hline & Herb m1 & Herb m2 & 4.73 & .001 & 31.3 \\
\hline & Herb m 2 & Herb m3 & 1.23 & 1.000 & 11.8 \\
\hline & Placebo m3 & Herb m3 & 16.13 & .000 & 63.8 \\
\hline & Baseline & Herb m3 & 22.73 & .000 & 64.5 \\
\hline \multirow[t]{5}{*}{ BDI } & Baseline & Herb m1 & 2.93 & .001 & 26.9 \\
\hline & Herb m1 & Herb m2 & .77 & .745 & 9.7 \\
\hline & Herb m 2 & Herb m3 & .67 & .766 & 9.31 \\
\hline & Placebo m3 & Herb m3 & 3.80 & .000 & 36.8 \\
\hline & Baseline & Herb m3 & 4.37 & .000 & 40.2 \\
\hline \multirow[t]{5}{*}{ ANX } & Baseline & Herb m1 & 3.17 & .007 & 12.2 \\
\hline & Herb m1 & Herb m2 & 1.57 & .147 & 6.9 \\
\hline & Herb $\mathrm{m} 2$ & Herb m3 & 1.03 & .622 & 4.8 \\
\hline & Placebo m3 & Herb m3 & 2.70 & .010 & 11.7 \\
\hline & Baseline & Herb m3 & 5.77 & .000 & 22.9 \\
\hline \multirow[t]{5}{*}{ ANG } & Baseline & Herb m1 & 3.37 & .001 & 18.6 \\
\hline & Herb m1 & Herb m2 & 1.37 & .815 & 9.3 \\
\hline & Herb m 2 & Herb m3 & 1.97 & .094 & 14.7 \\
\hline & Placebo $\mathrm{m} 3$ & Herb m3 & 4.63 & .000 & 28.8 \\
\hline & Baseline & Herb m3 & 6.70 & .000 & 31.9 \\
\hline \multirow[t]{5}{*}{ PSS } & Baseline & Herb m1 & 4.60 & .007 & 16.7 \\
\hline & Herb m1 & Herb m2 & 1.50 & .252 & 6.6 \\
\hline & Herb $\mathrm{m} 2$ & Herb m3 & .93 & .897 & 4.3 \\
\hline & Placebo m3 & Herb m3 & 5.63 & .006 & 21.6 \\
\hline & Baseline & Herb m3 & 7.03 & .000 & 24.8 \\
\hline
\end{tabular}


observed in any assessment scores. However, the comparisons between the baseline and end of the third herbal treatment month were highly significant ( $p$ $<.001$ ), as were the comparisons between the end of the placebo treatment month 3 and the final herbal treatment month $(\mathrm{p} \leqq .01)$ in all measurement scores favouring the active substance.

\subsubsection{Summary}

These findings indicate marked treatment effects on all clinical symptoms at the end of the herbal treatment in comparison to scores obtained before the commencement of treatment. Highly significant reductions were demonstrated in all assessment scores between baseline and the end of the final herbal treatment month $(\mathrm{p}<.001)$, and between the end of placebo treatment at month 3 and the last herbal treatment at month $6(\mathrm{p} \leqq .01)$ of the study. The largest herbal treatment effect on all clinical assessments occurred in the first month of the herbal treatment. The improvement then slowed down and the effect was still noticeable although not statistically significant in the last month.

From both comparisons between Group I and Group II, there were statistically significant differences in outcomes between prettest (baseline) and posttest (end of herbal treatment) during the herbal treatment period favouring the active herbal substances. 


\subsection{Follow-up of Premenstrual Physical and Psychological Symptoms}

The women in Group II received herbal treatment in the fourth, fifth and sixth months of the study. Following questionnaires were sent out by mail 10 weeks after the completion of the treatment, only 23 out of the 30 women in Group II returned their follow-up questionnaires (MDQ). The differences in treatment effects of herbal medicine on the composite premenstrual physical and psychological symptoms (PHYS, PSYC) of the MDQ at baseline, herbal treatment month 3 and three months after the completion of the treatment period (follow-up) of these 23 women were examined applying MANOVA .

The results revealed that there were significant differences $(\mathrm{p}<.01)$ between all these time phases and time by measures interactions (Table 9.11).

Table 9.11 Time and measures effects and measures by time interactions between baseline, herbal treatment month 3 and follow-up on physical and psychological symptoms of Group II (n=23)

\begin{tabular}{lcccc}
\hline Effect & \multicolumn{4}{c}{ Wilks' Lambda } \\
\hline \multirow{2}{*}{ Measures } & Value & $\mathrm{F}$ & Sig & Eta square \\
\cline { 2 - 5 } Time & .458 & $26.01(1,22)$ & .000 & .542 \\
Measures x Time & .121 & $75.99(2,21)$ & .000 & .879 \\
\hline
\end{tabular}

Further analysis using repeated measures ANOVA, on the composite physical and psychological symptoms was carried out to reveal the differential effects on physical and psychological symptoms. There were highly significant 
differences $(\mathrm{p}<.001)$ over the three time periods in scores of these two clinical symptom variables. These are shown in Table 9.12.

Table 9.12 Effects between baseline, herbal treatment month 3 and follow-up on physical and psychological symptoms of Group II $(n=23)$

\begin{tabular}{lcccc}
\hline Variable & Wilk's $\Lambda$ & $\mathrm{F}(2,21)$ & Sig & Eta square \\
\hline PHYS & .195 & 43.21 & .000 & .805 \\
PSYC & .138 & 65.38 & .000 & .862 \\
\hline
\end{tabular}

The results of the pairwise comparisons on time phases, shown in Table 9.13, indicate that there were highly significant reductions $(\mathrm{p}<.005)$ between baseline and follow up, but significant increases $(\mathrm{p}<.001)$ between herbal treatment month 3 and follow-up in scores of both physical and psychological symptoms.

Table 9.13 Comparisons between baseline, herbal treatment month 3 and follow-up of Physical and Psychological symptoms in Group II

\begin{tabular}{lllcl}
\multicolumn{2}{c}{$(\mathrm{n}=23)$} & & & \\
\hline & Time $(\mathrm{I})$ & Time $(\mathrm{J})$ & Mean Differences I-J & Sig \\
\hline PHYS & Baseline & h3 & 14.435 & .000 \\
& h3 & Follow-up & -7.652 & .000 \\
& Baseline & Follow-up & 6.783 & .002 \\
\hline PSYC & Baseline & h3 & 22.000 & .000 \\
& h3 & Follow-up & -9.391 & .000 \\
& Baseline & Follow-up & 12.609 & .000 \\
\hline
\end{tabular}


Treatment effects on physical and psychological symptoms across these three time phases are shown in Figure 9.5. Graphic descriptions of these two measures indicate that their mean scores at the follow-up period increased in comparison to the scores at the last month of the herbal treatment period. However, the scores at follow-up remained lower than the scores at baseline.

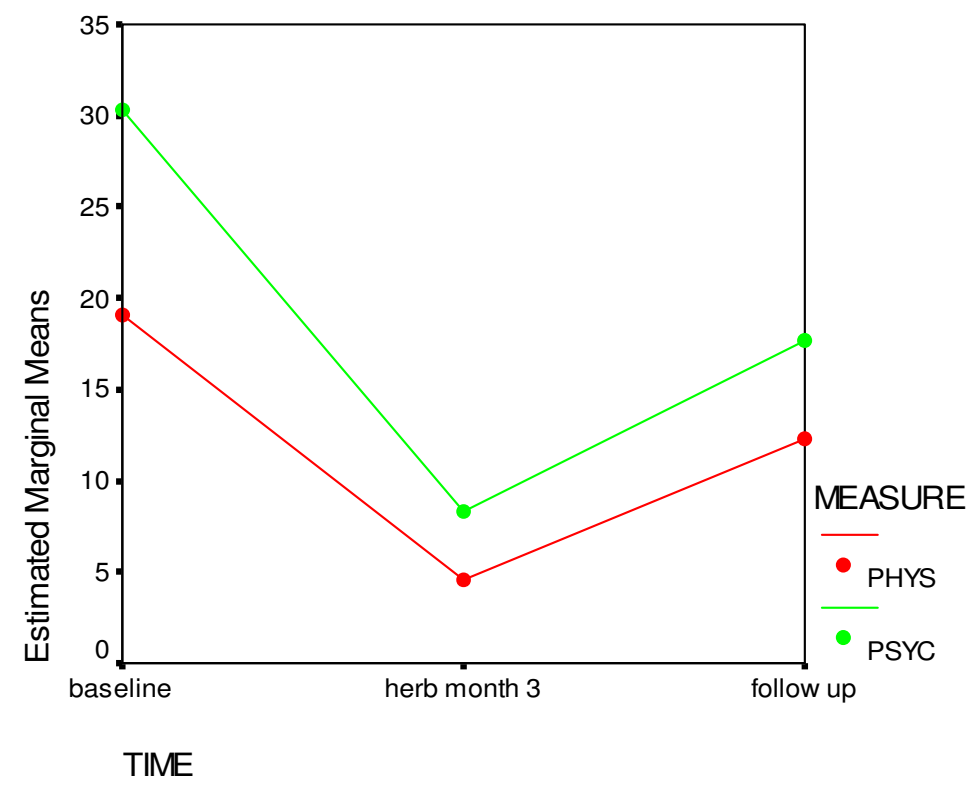

Figure 9.5 Mean scores at baseline, the third month of herbal treatment and follow-up on physical and psychological symptoms (MDQ) in Group II $(\mathrm{n}=23)$

In summary, the findings indicate that the herbal treatment effects on the composite physical and psychological symptoms of the MDQ at the follow-up period decreased in comparison to the effect at the last month of the herbal treatment period in this group of women. However, improvement was still maintained over pre-treatment in both symptoms. 


\subsection{Clinical Significance}

The significance of successful clinical treatment outcome was evaluated through examining the reduction in scores and the percentage of improvement between baseline and after treatment, and the calculation of the effect size between herbal and placebo treatment groups.

\subsubsection{Clinical Significance at the End of Herbal Treatment in Group I}

The mean scores of all clinical assessments on women of this group $(n=31)$ at baseline were examined for troubled and disordered groups (see Subdivision 4.4.2). The mean scores of the women in Group I on the composite premenstrual physical and psychological symptoms of the MDQ, depression and anger at the baseline were beyond one standard deviation (SD) of the mean of the normative group for that outcome measure, so the women of this group were recognized as a troubled group in these aspects before treatment. Significant clinical changes were reported only for those 'disturbed' according to the definition.

The mean scores of the composite premenstrual physical and psychological symptoms, depression and anger at herbal treatment month 3 were compared to the mean of the normative groups. The results indicate significant clinical changes (fallen to within $1 \mathrm{SD}$ of the mean of the norm) and over $77 \%$ women 
"having successful outcome" in these symptoms after the three months of herbal treatment.

The percent improvements in the composite physical and psychological symptoms, depression and anger between baseline and after herbal treatment were calculated. The results indicate that more than $39 \%$ improvements were showed in these symptoms in this group of women.

The mean effect sizes of these clinical assessments between baseline and end of herbal treatment were also calculated, and results indicate that effect sizes of these assessments were ranged from .90 to 2.48 . Clinically significant differences after herbal treatment of Group I are shown in Table 9.14.

Table 9.14 Significant clinical changes after three months herbal treatment

\begin{tabular}{|c|c|c|c|c|c|c|}
\hline \multirow[t]{2}{*}{ Var } & \multicolumn{3}{|c|}{$\operatorname{Mean}(\mathrm{SD})$} & \multirow{2}{*}{$\begin{array}{l}\text { Successful } \\
\text { outcome }\end{array}$} & \multirow{2}{*}{$\begin{array}{c}\% \\
\text { improvement }\end{array}$} & \multirow{2}{*}{$\begin{array}{r}\text { Effect }^{\triangle} \\
\text { Size }\end{array}$} \\
\hline & Norm* & Pre-treatment & Herbs-month 3 & & & \\
\hline PHYS & $6.31(4.82)$ & $20.71(7.99)^{3 / 2}$ & $6.45(4.21) * *$ & 83.9 & $68.9 * *$ & 2.23 \\
\hline PSYC & $10.38(7.61)$ & 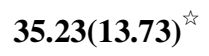 & $8.87(6.11) * *$ & 90.3 & $74.8 * *$ & 2.48 \\
\hline BDI & $4.73(4.73)$ & $10.87(5.10)^{\frac{12}{2}}$ & $6.19(5.28) * *$ & 77.4 & $43.3 * *$ & .90 \\
\hline ANX & $18.64(6.84)$ & $25.23(6.41)$ & $19.23(5.17)$ & & & \\
\hline ANG & 13.71(5.72) & $21.03(7.11)^{\sqrt[t h]{4}}$ & $12.77(2.85) * *$ & 96.8 & $39.3 * *$ & 1.52 \\
\hline PSS & $25.6(8.24)$ & $28.29(7.30)$ & $23.65(8.2)$ & & & \\
\hline
\end{tabular}

Troubled or disordered group * Clinically significant change

$\triangle .2=$ small effect, $.5=$ medium effect, $.8=$ large effect (Cohen, 1988)

*MDQ PHYS and PSYS (Moos 1985), BDI (Beck \& Beck, 1972))

*STPI State-Anxiety and State-Anger ( Spielberger 1979), PSS (Cohen, Kamarck \& Mermelstein, 1983) 


\subsubsection{Clinical Significance at the End of Herbal Treatment in Group II}

The mean scores on all clinical assessments of the women of this group $(n=30)$ at baseline were examined for troubled and disordered groups. The mean scores on the composite premenstrual physical and psychological symptoms, depression and anxiety at the baseline were beyond one standard deviation (SD) of the mean of the normative group for that outcome measure. Significant clinical changes were reported only for those 'disturbed' according to the definition.

The mean scores of the composite premenstrual physical and psychological symptoms, depression and anxiety at herbal treatment month 3 (month 6 of the study) in Group II were compared to the mean of the normative groups. The results indicate that significant clinical changes (fallen to within $1 \mathrm{SD}$ of the mean of the norm) and over than $76 \%$ women "having successful outcome" in these symptoms after the three months of herbal treatment.

The percent improvements in the composite physical and psychological symptoms, depression and anxiety between baseline and after herbal treatment were calculated. The results indicate that more than $40 \%$ improvements were showed in physical and psychological symptoms and depression, and only $22.1 \%$ improvement was shown in anxiety in this group of women.

The mean effect sizes of the composite physical and psychological symptoms, depression and anxiety between baseline and end of herbal treatment were also 
calculated, and results indicate that effect sizes of these assessments were ranged from .86 to 2.48 . Clinically significant differences after herbal treatment of Group II are shown in Table 9.15.

Table 9.15 Significant clinical changes after three months herbal treatment

\begin{tabular}{|c|c|c|c|c|c|c|}
\hline \multirow[t]{2}{*}{ Var } & \multicolumn{3}{|c|}{ Mean(SD) } & \multirow{2}{*}{$\begin{array}{l}\text { Successful } \\
\text { outcome }\end{array}$} & \multirow{2}{*}{$\begin{array}{c}\% \\
\text { improvement }\end{array}$} & \multirow{2}{*}{$\begin{array}{c}\text { Effect }^{\triangle} \\
\text { Size }\end{array}$} \\
\hline & Norm* & Pre-treatment & Herbs-month 3 & & & \\
\hline PHYS & $6.31(4.82)$ & $19.93(8.18)^{2 / 2}$ & $4.95(3.81) * *$ & 93.3 & $75.2 * *$ & 2.35 \\
\hline PSYC & $10.38(7.61)$ & $31.90(10.71)$ & $9.17(7.31) * *$ & 86.7 & $71.3 * *$ & 2.48 \\
\hline BDI & 4.73(4.73) & $10.90(5.58)^{\not / 2}$ & $6.53(4.55) * *$ & 76.7 & $40.1 * *$ & .86 \\
\hline ANX & $18.64(6.84)$ & $26.07(5.97)$ & $20.30(4.57)^{* * *}$ & 86.7 & 22.1 & 1.08 \\
\hline ANG & $13.71(5.72)$ & $18.13(5.31)$ & $11.43(1.31)$ & & & \\
\hline PSS & $25.6(8.24)$ & $27.50(8.50)$ & $20.47(7.36)$ & & & \\
\hline
\end{tabular}

\subsubsection{Clinical Significance at Follow-up}

The percent improvement in premenstrual physical symptoms and psychological at the follow-up month 3 in Group II remained greater than $35 \%$. The effect sizes of these two clinical assessments between the two time phases were .80 and 1.30, and over $52 \%$ "successful outcomes" showed in these two clinical assessments (see Table 9.16). Clinical changes remained significant at follow-up on premenstrual psychological symptoms which had fallen to within $1 \mathrm{SD}$ of the mean of the normative group. However, the mean scores of 
premenstrual physical symptoms though reduced, had not fallen to within $1 \mathrm{SD}$ from the norm mean.

Table 9.16 Clinically significant changes at follow-up of Group II (n=23)

\begin{tabular}{|c|c|c|c|c|c|c|}
\hline \multirow[t]{2}{*}{ Var } & \multicolumn{3}{|c|}{$\operatorname{Mean}($ SD) } & \multirow{2}{*}{$\begin{array}{l}\text { Successful } \\
\text { outcome }\end{array}$} & \multirow{2}{*}{$\begin{array}{c}\% \\
\text { improvement }\end{array}$} & \multirow{2}{*}{$\begin{array}{r}\text { Effect }^{\triangle} \\
\text { Size }\end{array}$} \\
\hline & Norm* & Pre-treatment & Follow-up & & & \\
\hline PHYS & $6.31(4.82)$ & $19.04(8.79)$ & $12.26(8.14)$ & 52.2 & $35.61 * *$ & .80 \\
\hline PSYC & $10.38(7.61)$ & $30.30(10.27)^{\text {th }}$ & $17.70(10.10) * *$ & 52.2 & $41.58 * *$ & 1.30 \\
\hline
\end{tabular}

\subsubsection{Summary}

These findings indicate that significant clinically relevant changes occurred with over $76 \%$ success rate, a large treatment effect size $(>.80)$ and over $39 \%$ improvement on premenstrual physical and psychological symptoms, depression and anger in participants of Group I $(n=31)$, and on premenstrual physical and psychological symptoms, depression and anxiety in participants of Group II ( $n=30)$ except only $22 \%$ improvement was shown on anxiety in Group II. However, the mean scores of perceived stress at baseline on women in both Group I and Group II indicate that they cannot be recognized as "disordered" groups, and as such clinical changes were not calculated. During the follow-up, over 35\% improvement and large effect size were maintained, and a 52.2\% success rate was achieved in both premenstrual physical and psychological symptoms in Group II. However, the mean score of the physical symptoms at follow-up was beyond one SD of the norm and was still within the range of “disordered". 
Chapter 10: Individual Symptoms Ratings, TCM Issues and Others $\quad$ Page

$\begin{array}{lll}\mathbf{1 0 . 1} \text { Individual Symptoms Ratings } & 244\end{array}$

10.1.1 Daily Symptoms Ratings 244

10.1.2 Comparison between MSD and MDQ Symptoms Ratings 245

10.1.3 Differences between Those Women Who Completed All 247

MSD Diaries and Those Who Did Not

$\begin{array}{lll}10.1 .4 & \text { Summary } 248\end{array}$

10.2 Relation between Patient Satisfaction and Treatment Outcome 248

10.3 Considerations of Treatment Effects by Ethnicity 250

$\begin{array}{llr}10.4 & \text { TCM Issues } & 250\end{array}$

10.4.1 TCM Patterns and Treatment Effect 250

10.4.2 Tongue and Pulses and Treatment Effect 253

10.4.2.1 Tongue Signs and Treatment Effect 253

10.4.2.2 Pulse Symptoms and Treatment Effect 254

10.4.2.3 Tongue Signs in TCM Patterns 256

10.4.2.4 Pulse Symptoms in TCM Pattern 258

$\begin{array}{lll}10.4 .3 & \text { Summary } & 259\end{array}$ 


\subsection{Individual Symptoms Ratings}

\subsubsection{Daily Symptoms Ratings}

Total scores of the daily symptoms ratings (DSR) recorded during the seven days prior to menstruation on each of 19 symptoms during the month before herbal treatment commenced and the three herbal treatment months were examined to investigate the treatment effects on these symptoms. Unfortunately, during the treatment period, most women failed to return a complete menstrual dairy for some months. Only 29 women in total (17 in Group I and 12 in Group II) charted their menstrual dairies completely in their premenstrual phases during the entire herbal treatment periods.

ANOVAs (analysis of variance) were performed on each of the 19 symptoms reported by these 29 women. The findings indicate that there was a highly significant reduction $(\mathrm{p}<.005)$ in nervous tension, mood swings, irritability, anxiety, breast tenderness, fatigue and depression, a significant reduction ( $\mathrm{p}$ $<.05)$ in swelling of extremities, increased appetite, crying, confusion and insomnia, and no significant difference in weight gain, abdominal bloating, headache, craving for sugar, heart pounding, dizziness and forgetfulness in the women of Group I ( $n=17)$. In the women of group II $(n=12)$, there was a highly significant reduction $(\mathrm{p}<.01)$ in irritability, anxiety, and fatigue, a significant reduction $(\mathrm{p}<.05)$ in nervous tension, swelling of extremities, breast tenderness, abdominal bloating, and insomnia, and no significant differences in 
mood swings, weight gain, headache, craving for sugar, heart pounding, dizziness, depression, forgetfulness, crying and confusion. The results are shown in Table 10.1. These findings must be considered with caution given the small numbers.

\subsubsection{Comparison between MSD and MDQ Symptoms Ratings}

Data recorded on the MDQ (Menstrual Distress Questionnaire) were collected retrospectively on Day 2 of the cycle for symptom severities in the week before menstruation (premenstrual phase) while data on the MSD (Menstrual Symptom Diary) were recorded every day prospectively. The scores of the 16 premenstrual symptoms in the MDQ (given that no abdominal bloating, sweet cravings and increased appetite are included in the MDQ) which were also assessed in the MSD of these 29 subjects, were calculated and compared to examine any difference in effect from herbal treatments between prospective and retrospective reports. Repeated measure ANOVAs were conducted on each of the 16 symptoms at baseline and the three herbal treatment months as scored of the MSD and the MDQ.

The results from the MSD and MDQ showed similarity in treatment effects emanating from herbs on both Groups I and Group II. In Group I, larger treatment effects were demonstrated on the MSD in nervous tension, mood swings, irritability, anxiety, breast tenderness, depression, crying and insomnia. Larger treatment effects were noted in the results of the MDQ in swelling of 
extremities, headache, fatigue, forgetfulness and confusion. In Group II, larger treatment effects were demonstrated in the MSD results on irritability, anxiety, swelling of extremities, breast tenderness, fatigue and insomnia. Larger treatment effects were noted on nervous tension, mood swings, weight gain, and depression. The results are shown in Table 10.1 .

Table 10.1 Treatment effects on premenstrual symptoms collected from MSD and MDQ at baseline and the three herbal treatment months in Group I and Group II

\begin{tabular}{|c|c|c|c|c|c|c|c|c|}
\hline \multirow{3}{*}{$\begin{array}{l}\text { Premenstrual } \\
\text { Symptoms }\end{array}$} & \multicolumn{4}{|c|}{ Group I (n=17) } & \multicolumn{4}{|c|}{ Group II (n=12) } \\
\hline & \multicolumn{2}{|c|}{ MSD } & \multicolumn{2}{|c|}{ MDQ } & \multicolumn{2}{|c|}{ MSD } & \multicolumn{2}{|c|}{ MDQ } \\
\hline & $F(3,14)$ & $\mathbf{P}$ & $\mathbf{F}(3,14$ & $\mathbf{P}$ & F $(3,9)$ & $\mathbf{P}$ & $F(3,27)$ & $\mathbf{P}$ \\
\hline Anxiety & 23.16 & $<.001$ & 2.84 & ns & 7.85 & $<.007$ & 1.72 & ns \\
\hline Mood swings & 16.62 & $<.001$ & 6.39 & $<.006$ & 3.68 & ns & 6.17 & $<.015$ \\
\hline Irritability & 15.92 & $<.001$ & 6.53 & $<.005$ & 6.94 & $<.010$ & 4.25 & $<.040$ \\
\hline Breast tenderness & 15.30 & $<.001$ & 6.84 & $<.005$ & 5.13 & $<.024$ & .76 & ns \\
\hline Fatigue & 8.79 & $<.002$ & 9.10 & $<.001$ & 11.41 & $<.002$ & 7.49 & $<.008$ \\
\hline Nervous tension & 8.79 & $<.002$ & 5.53 & $<.010$ & 3.91 & $<.049$ & 2.77 & $<.001$ \\
\hline Depression & 7.45 & $<.003$ & 5.96 & $<.008$ & 3.47 & ns & 8.18 & $<.006$ \\
\hline Crying & 4.91 & $<.015$ & 2.96 & ns & 2.72 & ns & .40 & ns \\
\hline Swelling of extremities & 4.69 & $<.018$ & 12.86 & $<.001$ & 4.13 & $<.043$ & .42 & ns \\
\hline Confusion & 4.60 & $<.019$ & 6.81 & $<.005$ & 2.88 & ns & 1.90 & ns \\
\hline Insomnia & 4.33 & $<.023$ & 3.34 & $<.050$ & 4.15 & $<.042$ & .32 & ns \\
\hline Increased appetite & 4.19 & $<.026$ & - & - & 2.68 & ns & - & \\
\hline Weight gain & 2.69 & ns & .57 & ns & 3.04 & ns & 4.32 & $<.038$ \\
\hline Dizziness or faintness & 2.58 & ns & 2.47 & ns & 1.07 & ns & 1.29 & ns \\
\hline Abdominal bloating & 2.25 & ns & - & - & 5.18 & $<.024$ & - & - \\
\hline Headache & 1.51 & ns & 3.46 & $<.046$ & 1.73 & ns & 1.60 & ns \\
\hline Heart pounding & 1.35 & ns & 1.60 & ns & .59 & ns & 1.67 & ns \\
\hline Forgetfulness & 1.24 & ns & 8.38 & $<.002$ & 2.32 & ns & 5.76 & ns \\
\hline Craving for sweet & .55 & ns & - & - & 2.00 & ns & - & \\
\hline
\end{tabular}

$* \mathrm{~ns}=\mathrm{p}>.05$ 


\subsubsection{Differences between Those Women Who Completed All MSD Diaries and Those Who Did Not}

Only 29 out of the sixty one women in the study managed to chart and return their menstrual dairies completely during the entire herbal treatment periods. ANOVA were performed on each of the assessment scores at baseline to compare the differences between those women $(n=29)$ who completed their charting of premenstrual symptoms in the menstrual diaries and those $(n=32)$ who failed to complete all diaries. The findings (Table 10.2) indicate that there were no significant differences $(\mathrm{p}>.05)$ in all assessment scores except for depression at the baseline between the two groups of women. Women who failed to complete charting their premenstrual symptoms in all menstrual diaries during the herbal treatment period in the study showed higher scores on the depression questionnaire (BDI) than the other group of women.

Table 10.2 Comparison of baseline scores between women who completed all MSD diaries and those not

\begin{tabular}{ccccc}
\hline Baseline scores & $\begin{array}{c}\text { Complete all diaries } \\
(\mathrm{n}=29)\end{array}$ & $\begin{array}{c}\text { Not complete diaries } \\
(\mathrm{n}=32)\end{array}$ & $\mathrm{F}$ & Sig. \\
\hline ANG & 20.55 & 18.75 & 1.21 & .276 \\
ANX & 25.55 & 25.72 & .011 & .917 \\
BDI & 9.41 & 12.22 & 4.51 & .038 \\
PHYS & 19.14 & 21.41 & 1.22 & .274 \\
PSS & 26.07 & 29.56 & 3.12 & .083 \\
PCYS & 31.55 & 35.44 & 1.52 & .223 \\
\hline
\end{tabular}




\subsubsection{Summary}

The findings from the MSD indicate that the largest herbal treatment effects were recorded on irritability, anxiety and fatigue ( $\mathrm{p} \leqq .01)$, followed by breast tenderness, nervous tension, swelling of extremities and insomnia ( $p$ $<.05)$ in both groups. The herbal treatment effects on premenstrual symptoms shown in the prospective report (i.e. MSD) and the retrospective report (i.e. MDQ) were very similar, and the similarity was more prominent when the sample size was larger in Group I.

\subsection{Relation between Patient Satisfaction and Treatment Outcome}

Kendall's Tau-b correlation coefficient was applied to examine the relation between the clinical assessment scores and the clients' satisfaction rating on the third and sixth month of the experimental period.

All correlations reported in Table 10.3a and Table $10.3 \mathrm{~b}$ were highly

significant $(\mathrm{p}<.01)$. The women's satisfaction rating was negatively related to all clinical assessment scores. Thus, the higher the rating of the clients' satisfaction the better was the treatment outcome in terms of their satisfaction.

The mean of the total group $(n=61)$ of women satisfaction rating which was recorded in the third month of herbal treatment was compared, using one sample t-test, to the rating of the third month of placebo treatment. The result 
shows that there were highly significant differences $(\mathrm{p}<.001)$ between the ratings at these two time phases, and the mean rating at the herbal treatment month 3 was higher than the mean rating at the placebo treatment month 3 .

This finding indicates that herbal treatment achieved a better treatment outcome than the placebo and was reflected in the women's self reports of satisfaction with the treatment received.

Table 10.3a Correlation between clients' satisfaction rating and clinical assessment scores at the third month of experimental period $(\mathrm{n}=61)$

\begin{tabular}{llcccccc}
\hline & & $\begin{array}{c}\text { Physical } \\
\text { symptoms }\end{array}$ & $\begin{array}{c}\text { Psychological } \\
\text { symptoms }\end{array}$ & Depression & Anxiety & Anger & $\begin{array}{c}\text { Perceived } \\
\text { Stress }\end{array}$ \\
\hline Clients' & Kendall's & -.452 & -.583 & -.366 & -.421 & -.257 & -.282 \\
satisfaction & tau-b & & & & & & \\
rating & Sig & .000 & .000 & .000 & .000 & .010 & .004 \\
& (2-tailed) & & & & & & \\
\hline
\end{tabular}

Table 10.3b Correlation between clients' satisfaction rating and clinical assessment scores at the sixth month of experimental period

\begin{tabular}{llcccccc}
\multicolumn{1}{c}{$(\mathrm{n}=61)$} & & & & \\
\hline & & $\begin{array}{c}\text { Physical } \\
\text { symptoms }\end{array}$ & $\begin{array}{c}\text { Psychological } \\
\text { symptoms }\end{array}$ & Depression & Anxiety & Anger & $\begin{array}{c}\text { Perceived } \\
\text { Stress }\end{array}$ \\
\hline $\begin{array}{l}\text { Clients' } \\
\text { satisfaction } \\
\text { rating }\end{array}$ & Kendall's & -.454 & -.537 & -.363 & -.326 & -.453 & -.443 \\
& tau-b & & & & & & \\
& Sig & .000 & .000 & .000 & .001 & .000 & .000 \\
& & & & & & & \\
\hline
\end{tabular}




\subsection{Considerations of Treatment Effects by Ethnicity}

Ethnicity was recoded into two groups, i.e. women of group A $(n=37)$ were English-speaking Australasian or British, and women in group B ( $\mathrm{n}=24)$ were European or Asian. The clinical assessment scores at each month of the herbal treatment period were investigated using repeated measures ANCOVA (analysis of covariance) on the two ethnic groups to consider whether any ethnic effect might have influenced the treatment result.

There was no significant effect on any assessment or time phase for the ethnic groups [Wilks' Lambda $=.858, \mathrm{~F}(10,50)=.826, \mathrm{p}>.5$ ], and there was also no significant difference [Greenhouse-Geisser $\mathrm{F}(50146,303.641)=.975, \mathrm{p}>.05$ ] between all clinical assessments and between all time phases in the univariate test.

\subsection{TCM Issues}

\subsubsection{TCM Patterns and Treatment Effect}

Repeated measures MANOVA (multivariate analysis of variance) was carried out to examine the score differences between TCM patterns at the three months of herbal treatment period. Data from PHYS (Composite scores of physical symptoms), PSYC (Composite scores of psychological symptoms), BDI (depression), ANX (anxiety), ANG (anger), and PSS (perceived stress) 
collected in the herbal treatment period of the study on women of the four patterns (see Subdivision 8.6.2), namely Pattern A ( $n=18)$, Pattern CA ( $n=10)$, Pattern H (n=21) and Pattern D (n=12) were used for analysis.

The multivariate analysis showed that there were no significant differences ( $p$ $>.05$ ) between patterns over times, and patterns over times by measures (Table 10.4).

Table 10.4 Time, Measure and Pattern interactions between three herbal treatment month $(n=61)$

\begin{tabular}{lrrrc}
\hline Effect & \multicolumn{5}{c}{ Wilks' Lambda } \\
\hline \multirow{2}{*}{ Time } & Value & F & Sig & Eta square \\
\cline { 2 - 5 } Measure & .397 & $42.59(2,56)$ & .000 & .603 \\
Measure x Pattern & .051 & $197.60(5,53)$ & .000 & .949 \\
Time x Pattern & .622 & $1.84(15,146)$ & .035 & .146 \\
Measure x Time x Pattern & .825 & $1.88(6,112)$ & .090 & .092 \\
\hline
\end{tabular}

Further analysis was carried out using pairwise comparisons on treatment effects between TCM patterns at the three months of herbal treatment condition. The result indicates that there were no significant differences between any of these patterns at the three months of herbal treatment condition. Women of Pattern A had the largest treatment effect from herbal treatment, Pattern $\mathrm{H}$ and CA ranked second and third respectively, and women of Pattern D had the smallest effect (Table 10.5a).

Follow up analysis using pairwise comparisons were also performed to evaluate the changes in scores on all clinical assessments at the end of the herbal 
treatment condition. The results indicate that there were no significant

differences between any of these patterns at the end of the herbal treatment condition (Table 10.5b).

Table 10.5a Treatment effects between TCM patterns at the three months of herbal treatment condition $n=61$

\begin{tabular}{cccr}
\hline Pattern $(\mathrm{I})$ & Pattern $(\mathrm{J})$ & Mean Differences I-J & Sig \\
\hline A & H & -1.52 & 1.000 \\
A & CA & -2.73 & .591 \\
A & D & -4.18 & .051 \\
H & CA & -1.21 & 1.000 \\
H & D & -2.66 & .474 \\
CA & D & -1.453 & 1.000 \\
\hline
\end{tabular}

Table 10.5b Scores differences of all measures between TCM patterns at the end of herbal treatment condition (herbal treatment month 3) $n=61$

\begin{tabular}{|c|c|c|c|c|c|c|c|c|c|c|c|c|c|}
\hline \multirow{3}{*}{$\begin{array}{l}\text { Pattern } \\
\text { (I) }\end{array}$} & \multirow{3}{*}{$\begin{array}{l}\text { Pattern } \\
\text { (J) }\end{array}$} & \multicolumn{2}{|c|}{ PHYS } & \multicolumn{2}{|c|}{ PSYC } & \multicolumn{2}{|c|}{ BDI } & \multicolumn{2}{|c|}{ ANX } & \multicolumn{2}{|c|}{ ANG } & \multicolumn{2}{|c|}{ PSS } \\
\hline & & Mean & & Mean & & Mean & & Mean & & Mean & & Mean & \\
\hline & & I-J & Sig & I-J & Sig & I-J & Sig & I-J & Sig & I-J & Sig & I-J & Sig \\
\hline A & CA & -2.933 & 374 & -5.156 & .286 & -3.756 & .295 & .144 & 1.000 & 1.011 & 1.000 & -3.967 & 1.000 \\
\hline A & D & -3.583 & .102 & -4.806 & .304 & -4.056 & .152 & -1.889 & 1.000 & -1.306 & .817 & -1.583 & 1.000 \\
\hline A & $\mathrm{H}$ & -2.524 & .296 & -.817 & 1.000 & -1.460 & 1.000 & -1.341 & 1.000 & .254 & 1.000 & .619 & 1.000 \\
\hline CA & $\mathrm{D}$ & -.650 & 1.000 & .350 & 1.000 & -.300 & 1.000 & -2.033 & 1.000 & -2.317 & .139 & 2.383 & 1.000 \\
\hline $\mathrm{CA}$ & $\mathrm{H}$ & .410 & 1.000 & 4.338 & .515 & 2.295 & 1.000 & -1.486 & 1.000 & -.757 & 1.000 & 4.586 & .826 \\
\hline D & $\mathrm{H}$ & 1.060 & 1.000 & 3.988 & .560 & 2.595 & .813 & .548 & 1.000 & 1.560 & .409 & 2.202 & 1.000 \\
\hline
\end{tabular}




\subsubsection{Tongue and Pulses and Treatment Effect}

\subsubsection{Tongue Signs and Treatment Effect}

The data on tongue coatings and tongue bodies before and after herbal treatment were calculated and compared. After three months of herbal treatment, general improvement in tongues signs was observed in the current study. About $90 \%$ of women had a pink tongue with thin and white tongue coating which is recognized as a tongue with normal conditions in TCM, and none of them had a red or dark red tongue that signifies a heat sign of the body in TCM. Purplish areas on the tongue which indicate stagnation in the body were reduced from $41 \%$ to $8.2 \%$ ( $80 \%$ improvement). The teeth marks which indicate retention of water were reduced from $44.3 \%$ to $27.9 \%$ (37\% improvement). Swollen tongues which indicate dampness retention were reduced from $26.6 \%$ to $11.5 \%$ (56.3\% improvement), and vertical cracks on the tongue which indicate deficiency of blood, Yin or Stomach $Q i$ were reduced from 21 cases to 11 cases (47.6\% improvement). All tongue signs before and after herbal treatment are shown in Table 10.6. Paired t-tests were conducted to compare the differences between pretest and posttest on tongue colour, colour and thickness of tongue coating. All results revealed significant differences ( $p$ $<.05)$ between baseline and after herbal treatment. 
Table 10.6 Comparison between baseline and after herbal treatment on

\begin{tabular}{|c|c|c|c|c|c|c|c|}
\hline \multirow[b]{2}{*}{ Tongue } & & \multicolumn{2}{|c|}{ Baseline } & \multicolumn{2}{|c|}{ Herbs month 3} & \multirow{2}{*}{$\begin{array}{c}\% \\
\text { improvement }\end{array}$} & \multirow[t]{2}{*}{ Sig. } \\
\hline & & $\mathbf{N}$ & $\%$ & $\mathbf{N}$ & $\%$ & & \\
\hline \multicolumn{8}{|l|}{ Coating: } \\
\hline \multirow[t]{3}{*}{ Thickness } & Moderate & 18 & 29.5 & 7 & 11.5 & & .038 \\
\hline & Thin & 41 & 67.2 & 54 & 88.5 & & \\
\hline & Thick & 2 & 3.3 & $\mathbf{0}$ & $\mathbf{0}$ & & \\
\hline \multirow[t]{2}{*}{ Colour } & White & 41 & 67.2 & 57 & 93.5 & & .003 \\
\hline & Yellow & 20 & 32.7 & 4 & 6.6 & 80.0 & \\
\hline Abnormal & Wet/slippery & 4 & 6.6 & 1 & 1.6 & 75.0 & NA \\
\hline \multirow[t]{2}{*}{ Proper } & Dry & 1 & 1.6 & 1 & 1.6 & $\mathbf{0}$ & \\
\hline & Peeled/map & 5 & 8.2 & 1 & 1.6 & 80.0 & \\
\hline \multicolumn{8}{|l|}{ Body: } \\
\hline \multirow[t]{4}{*}{ Colour } & Pink & 42 & 68.9 & 57 & 93.4 & & .000 \\
\hline & Pale & 8 & 13.1 & 4 & 6.6 & & \\
\hline & Red & 9 & 14.8 & $\mathbf{0}$ & $\mathbf{0}$ & & \\
\hline & Dark red & 2 & 3.3 & $\mathbf{0}$ & $\mathbf{0}$ & & \\
\hline Abnormal & Red Side & 10 & 16.4 & 6 & 9.8 & 40.0 & NA \\
\hline \multirow[t]{7}{*}{ Appearance } & Red Tip & 10 & 16.4 & 6 & 9.8 & 40.0 & \\
\hline & Teeth mark & 27 & 44.3 & 17 & 27.9 & 37.0 & \\
\hline & Swollen & 16 & 26.2 & 7 & 11.5 & 56.3 & \\
\hline & Thin \& small & 3 & 4.9 & 1 & 1.6 & 66.6 & \\
\hline & Cracks (V) & 21 & 34.4 & 11 & 18.0 & 47.6 & \\
\hline & Crack $(T)$ & 1 & 1.6 & 1 & 1.6 & $\mathbf{0}$ & \\
\hline & Purplish area & 25 & 41.0 & 5 & 8.2 & 80.0 & \\
\hline
\end{tabular}

\subsubsection{Pulse Symptoms and Treatment Effect}

The data on pulses before and after herbal treatment were calculated and compared. General improvement in pulse symptoms was again observed at the 
end of the herbal treatment in the current study. There was a $60.4 \%$ reduction in the presentation of slippery pulse which indicates retention of dampness and heat in TCM, a $49.1 \%$ reduction in the presentation of choppy pulse which indicates $Q i$ stagnation or deficiency of $Q i$, a $38.6 \%$ reduction in the presentation of thin pulse which indicates deficiency of $Q i$ or retention of dampness and a $15.6 \%$ reduction in the presentation of wiry pulse which indicates Liver $Q i$ stagnation or diseases in the Liver system.. There was not any rapid pulse which indicates a heat or deficiency syndrome, weak pulse which indicates deficiency of $Q i$ and blood, or scattered pulse which indicates exhaustion of $Q i$ or deficiency of $Q i$ and blood detected at the end of herbal treatment. All pulses recorded at the first interview and at the end of herbal treatment are shown in Table 10.7.

Table 10.7 Comparison between baseline and after herbal treatment on Pulses $(n=61)$

\begin{tabular}{|c|c|c|c|c|c|}
\hline \multirow[b]{3}{*}{ Pulses } & ses & $=61)$ & & & \\
\hline & \multicolumn{2}{|c|}{ Baseline } & \multicolumn{2}{|c|}{ Herbal month 3} & \multirow{2}{*}{$\begin{array}{c}\text { Less } \\
\%\end{array}$} \\
\hline & $\mathbf{N}$ & $\%$ & $\mathbf{N}$ & $\%$ & \\
\hline Wiry & 58 & 95.1 & 49 & 80.3 & 15.6 \\
\hline Thin & 44 & 72.1 & 27 & 44.3 & 38.6 \\
\hline Slippery & 43 & 70.5 & 17 & 27.9 & 60.4 \\
\hline Choppy & 18 & 29.5 & 9 & 14.8 & 49.1 \\
\hline Rapid & 8 & 13.1 & 0 & - & 100 \\
\hline Weak & 4 & 6.6 & 0 & - & 100 \\
\hline Scattered & 1 & 1.6 & 0 & - & 100 \\
\hline
\end{tabular}




\subsubsection{Tongue Signs in TCM Patterns}

Over $80 \%$ of the women in Pattern A (Liver Qi Stagnation) of the study had a pink tongue and a thin coating, $72.2 \%$ had a white coating, $44.4 \%$ had vertical cracks, $27.7 \%$ had teeth marks, and $22.2 \%$ had red tips and purplish areas on their tongues at the first interview. After three months of herbal treatment, all these women had a pink tongue, about $90 \%$ had thin and white coating, $11.1 \%$ had purplish areas, one third of them still had vertical cracks, one quarter had teeth marks and no change on red tips of their tongues.

In pattern CA (Liver Qi Stagnation, Liver and Kidney Yin Deficiency), half of the women sufferers had a pale tongue, $40 \%$ had a red tongue and $10 \%$ had a tongue of dark red in colour. Eighty percent of them had thin and yellowish coating, $70 \%$ had purplish areas, and $40 \%$ had vertical cracks and red sides on their tongues at the first interview. After three months herbal treatment, all these women had a pink tongue with thin and white coating, no one had purplish area while $10 \%$ still had vertical cracks and $30 \%$ still had red sides on their tongues.

In pattern D (Liver Qi Stagnation, Heart and Spleen Deficiency), 75\% of the women sufferers had a pale tongue and white coating, $50 \%$ had moderate and 50\% had thin coating. $41.7 \%$ had a swollen tongue and purplish areas, $33.3 \%$ had red tip and teeth marks on their tongues at the first interview. At the end of the herbal treatment, $91.7 \%$ of these women had a pink tongue, and $83.3 \%$ had 
a thin and white coating. Swollen tongues and red tips and purplish areas of their tongues were reduced to $16.6 \%$, however, no changed was observed on teeth marks of their tongues.

In pattern H (Liver Qi Stagnation, Liver and Spleen Deficiency causing fluid retention), $61.9 \%$ of the women suffers had a pink tongue, $23.8 \%$ had a red tongue and $14.2 \%$ had a pale tongue, $81 \%$ had white coating, $57.1 \%$ had thin coating, $42.9 \%$ had moderate coating, $71.4 \%$ had teeth marks, $42.9 \%$ had purplish areas on their tongues and $38.1 \%$ had a swollen tongue at the first interview. After herbal treatment, over $85 \%$ had a pink tongue with thin white coating, only $4.8 \%$ had purplish areas on their tongues, and swollen tongues and teeth marks had around 50\% reduction. All tongue signs in the four TCM patterns are shown in Table 10.8 and Table 10.9.

Table 10.8 Tongue coating in TCM patterns before and after herbal treatment

\begin{tabular}{|c|c|c|c|c|c|c|c|c|c|c|c|c|c|c|c|c|}
\hline \multirow{3}{*}{$\begin{array}{l}\text { Pattern (n) } \\
\text { Before/After }\end{array}$} & \multicolumn{6}{|c|}{ Thickness } & \multicolumn{4}{|c|}{ Colour } & \multicolumn{6}{|c|}{ Proper } \\
\hline & \multicolumn{2}{|c|}{ Mod } & \multicolumn{2}{|c|}{ Thin } & \multicolumn{2}{|c|}{ Thick } & \multicolumn{2}{|c|}{ White } & \multicolumn{2}{|c|}{ Yellow } & \multicolumn{2}{|c|}{ Wet } & \multicolumn{2}{|c|}{ Dry } & \multicolumn{2}{|c|}{ peeled } \\
\hline & B & $\mathbf{A}$ & B & $\mathbf{A}$ & B & $\mathbf{A}$ & B & $\mathbf{A}$ & B & $\mathbf{A}$ & B & $\mathbf{A}$ & B & & B & $\mathbf{A}$ \\
\hline A (18) & 2 & 2 & 15 & 10 & 1 & 0 & 13 & 17 & 5 & 1 & 2 & 1 & 1 & & 2 & $\mathbf{0}$ \\
\hline CA (10) & 1 & 0 & 8 & 10 & & 0 & 2 & 10 & 8 & 0 & 1 & 0 & 0 & & 1 & $\mathbf{0}$ \\
\hline $\mathrm{D}(12)$ & 6 & 2 & 6 & 10 & 8 & 0 & 9 & 10 & 3 & 2 & 1 & 0 & $\mathbf{0}$ & & $\mathbf{0}$ & $\mathbf{0}$ \\
\hline $\mathrm{H}(21)$ & 9 & 3 & 12 & 18 & $\mathbf{0}$ & 0 & 17 & 20 & 4 & 1 & $\mathbf{0}$ & 0 & $\mathbf{0}$ & 0 & 2 & 1 \\
\hline
\end{tabular}


Table 10.9 Tongue body in TCM patterns before and after herbal treatment

\begin{tabular}{|c|c|c|c|c|c|c|c|c|c|c|c|c|c|c|c|c|c|c|c|c|c|c|c|c|}
\hline \multirow{3}{*}{$\begin{array}{l}\text { Pattern } \\
\text { (n) } \\
\text { B/A }\end{array}$} & \multicolumn{8}{|c|}{ Colour } & \multicolumn{16}{|c|}{ Appearance } \\
\hline & \multicolumn{2}{|c|}{ Pink } & \multicolumn{2}{|c|}{ Pale } & \multicolumn{2}{|c|}{ Red } & \multicolumn{2}{|c|}{ Dark red } & \multicolumn{2}{|c|}{ Swollen } & \multicolumn{2}{|c|}{$\begin{array}{c}\text { Thin \& } \\
\text { small }\end{array}$} & \multicolumn{2}{|c|}{$\begin{array}{c}\text { Cracks } \\
\text { (V) }\end{array}$} & \multicolumn{2}{|c|}{$\begin{array}{c}\text { Cracks } \\
\text { (T) }\end{array}$} & \multicolumn{2}{|c|}{ Red side } & \multicolumn{2}{|c|}{ Red tip } & \multicolumn{2}{|c|}{$\begin{array}{l}\text { Teeth } \\
\text { mark }\end{array}$} & \multicolumn{2}{|c|}{$\begin{array}{c}\text { Purplish } \\
\text { area }\end{array}$} \\
\hline & B & A & B & A & B & A & B & A & B & $\mathbf{A}$ & B & A & B & A & B & A & B & A & B & A & B & A & B & $\mathbf{A}$ \\
\hline A (18) & 15 & 18 & 1 & 0 & 1 & 0 & 1 & $\mathbf{0}$ & 3 & o & 2 & 2 & 8 & 6 & 1 & 1 & 3 & 2 & 4 & 4 & 5 & 4 & 4 & 2 \\
\hline CA (10) & 0 & 10 & 5 & 0 & 4 & 0 & 1 & 0 & 0 & 1 & 0 & 0 & 4 & 1 & $\mathbf{0}$ & 0 & 4 & 3 & $\mathbf{0}$ & 0 & 3 & 1 & 7 & 0 \\
\hline D (12) & 2 & 11 & 9 & 1 & 1 & 0 & 0 & 0 & 5 & 2 & 0 & 0 & 3 & 1 & 0 & 0 & 1 & 0 & 4 & 2 & 4 & 4 & 5 & 2 \\
\hline H (21) & 13 & 18 & 5 & 3 & 3 & 0 & 0 & 0 & 8 & 4 & 1 & 0 & 6 & 3 & o & 0 & 2 & 1 & 2 & 0 & 15 & 8 & 9 & 1 \\
\hline
\end{tabular}

\subsubsection{Pulse Symptoms in TCM Patterns}

These PMS women sufferers in all patterns had a very high percentage of wiry pulse ranging from $83.3 \%$ to $100 \%$ at their first interview. All women in Pattern CA and $\mathrm{H}$ had wiry pulses, $91.7 \%$ of the women in Pattern D had thin pulses, $76.2 \%$ of the women in Pattern $\mathrm{H}$ had slippery pulses, and $33.3 \%$ of the women in Pattern A and D had choppy pulses. A 100\% reduction was observed in rapid pulses and weak pulses in all four TCM Patterns, so was scattered pulse and choppy pulse in Pattern D after herbal treatment. There was a 76.9\% reduction and a $62.5 \%$ reduction of slippery pulses in Pattern A and $\mathrm{H}$ respectively. However, only about $10 \%$ reduction was achieved in wiry pulses in Pattern A and D after herbal treatment, no reduction in Pattern CA and about $30 \%$ reduction in Pattern $\mathrm{H}$. All pulse symptoms before and after treatment are shown in Table 10.10 . 
Table 10.10 Pulse symptoms in TCM patterns before and after herbal

\begin{tabular}{|c|c|c|c|c|c|c|c|c|c|c|c|c|c|c|}
\hline \multirow{2}{*}{$\begin{array}{l}\text { Pattern (n) } \\
\text { Before/After }\end{array}$} & \multicolumn{2}{|c|}{ Wiry } & \multicolumn{2}{|c|}{ Thin } & \multicolumn{2}{|c|}{ Slippery } & \multicolumn{2}{|c|}{ Choppy } & \multicolumn{2}{|c|}{ Rapid } & \multicolumn{2}{|c|}{ Weak } & \multicolumn{2}{|c|}{ Scattered } \\
\hline & B & A & B & A & B & A & B & A & B & A & B & A & B & A \\
\hline A (18) & 17 & 15 & 12 & 8 & 13 & 3 & 6 & 5 & 2 & 0 & 1 & 0 & 0 & o \\
\hline CA (10) & 10 & 10 & 8 & 3 & 6 & 3 & 2 & 1 & 1 & 0 & 1 & 0 & 0 & 0 \\
\hline $\mathrm{D}(12)$ & 10 & 9 & 11 & 7 & 8 & 5 & 4 & 0 & 3 & O & 1 & O & 1 & 0 \\
\hline $\mathrm{H}(21)$ & 21 & 15 & 13 & 9 & 16 & 6 & 6 & 3 & 2 & 0 & 1 & 0 & 0 & 0 \\
\hline
\end{tabular}

\subsubsection{Summary}

There were no significant differences $(p>.05)$ shown in the treatment effects of all clinical assessments between the various TCM patterns. However, women of Pattern A had the largest treatment effect from herbal treatment.

Significant improvements $(\mathrm{p}<.05)$ were shown in the thickness of tongue coating, colour of the tongue coating and body after three months of herbal treatment. A fairly high percentage of reductions was observed in most of the abnormal appearance of the tongue body and coating proper after three months of herbal treatment in the four TCM pattern groups and the pooled group. Marked reductions were observed in abnormal pulses including wiry, slippery, choppy, thin, rapid and weak pulses by the end of the herbal treatment in the four TCM pattern groups and the pooled group. Wiry pulses showed the smallest treatment effect after herbal treatment. 
11.2.1 Hypothesis $1 \quad 261$

11.2.2 Hypothesis 2

11.2.3 Hypothesis 3 265

11.2.4 Hypothesis $4 \quad 267$

$\begin{array}{lll}\text { 11.3 Adverse Effects of Herbs } & 268\end{array}$

$\begin{array}{llr}11.4 & \text { Placebo Effect } & 269\end{array}$

$\begin{array}{ll}\text { 11.5 The TCM Patterns of Disharmony } & 271\end{array}$

11.6 TCM Symptom Patterns and Western Perspective Symptom 272 Patterns

$\begin{array}{lll}\text { 11.7 Signs and Symptoms on Tongue and Pulses } & 274\end{array}$

$\begin{array}{lll}11.8 & \text { Sample characteristics } & 278\end{array}$

$\begin{array}{lll}11.9 & \text { Menstrual Characteristics } & 281\end{array}$

$\begin{array}{lll}11.10 & \text { Individual Symptoms Ratings } & 282\end{array}$

11.11 Prospective and Retrospective Reports of Symptoms 283

$\begin{array}{ll}\text { 11.12 Alcohol and smoking } 285 & 285\end{array}$

11.13 Ethnicity and Treatment Efficacy 286

$\begin{array}{lll}11.14 & \text { Subject Attrition } & 287\end{array}$

$\begin{array}{lll}11.15 & \text { Limitations } & 288\end{array}$

$\begin{array}{llr}11.16 & \text { Summary } & 289\end{array}$ 


\subsection{Overview}

The findings of the study provide positive support both statistically and clinically regarding the effectiveness of Chinese herbal medicine for Premenstrual Syndrome (PMS) symptoms on a sample of Australian women in that the posttest means of symptoms occurrence and severity in the experimental (Chinese herbal medicine) groups were lower than the pretest means; and the reduction in symptoms occurrence and severity of PMS was maintained at three month follow-up. The findings also indicate that herbal treatment achieved a better treatment outcome than the placebo in that the posttest means of symptoms occurrence and severity in the experimental (Chinese herbal medicine) groups were lower than the control (placebo) group. The effectiveness of the treatment was further reflected in the women's self reports of satisfaction with the treatment received. All hypotheses of the study have been positively supported by these findings.

\subsection{Hypothesis}

\subsubsection{Hypothesis 1, the posttest means of the symptoms occurrence and severity from herbal medicine treatment condition will be lower than the pretest means.}


This was supported in that, when significant differences were observed between baseline and experimental phases in the clinical assessment results of both Group I and Group II. At the end of the herbal treatment there were highly significant improvements in all six clinical assessments in the women from Group I and II, including physical and psychological symptoms, depression, anxiety, anger and perceived stress.

The findings of the current study have significantly demonstrated that there was marked improvement at the end of the first month of the herbal treatment. Gradual and steady improvements continued throughout the next two months leading to a substantial reduction in symptoms on completion of the herbal treatment. These findings indicate that Chinese herbal medicine produces an obvious treatment effect on premenstrual symptoms. Clearly, the largest treatment effect was shown in the first month, but the full treatment effect takes time to be realized and felt by the patient. This phenomenon illustrates one of the main principles of TCM treatment which is to restore the function of the Zang-Fu organs by readjusting the balance of yang and yin of the body (Liu et al, 1995). It is because Chinese medicine believes that the human body is an inseparable organic whole and the functions of all the internal organs are interconnected and interdependent. This is known as the Five Elements theory of TCM (Anonymous, 100BC). In the case of PMS, which has a number of physical and/or psychological symptoms, it is quite common that more than one of the Zang-Fu organs is involved. The body may need some time for the pharmaceutical elements to exert their effects, to regulate the imbalance in the Zang-Fu organs, and to restore equilibrium of yin and yang in the entire body. 
The outcomes of the current study also suggest that the significant clinical improvements made between baseline and the end of herbal treatment on premenstrual physical and psychological symptoms positively affected negative emotions of depression, anxiety and anger. The post herbal treatment mean scores on these assessments all fell to within one standard deviation of the mean of the normative groups, which demonstrated significant clinical changes (Becker 1977). The finding also indicates that over $75 \%$ of women, whose post treatment mean scores on these assessments fell to within one standard deviation of the mean of their untroubled peers, can be viewed as having a "successful outcome" (Becker 1977) in symptom reduction after the herbal treatment.

The percent improvements made between baseline and after herbal treatment in premenstrual physical and psychological symptoms, and depression after herbal treatment were ranged between $39-73 \%$. This result also indicates that there were clinically significant differences (Long, 1995) between these two time phases in these symptoms. The mean effect sizes of these clinical assessments between baseline and end of herbal treatment ranged from .86 to 2.48 which also represents a large clinically significant difference according to Cohen's (1988) definition. 


\subsubsection{Hypothesis 2, the posttest means of the symptoms and their severity in the Chinese herbal medicine treatment group will be lower than the control (placebo) group.}

This was supported as significant differences were observed between Group I (Chinese herbal medicine) and Group II (placebo) at the end of the third month of the study, and between the third month (placebo) and the sixth month (Chinese herbal medicine) of the study in Group II. In the first scenario, significant differences were noted between Groups I and II at the end of the third month of the study on the outcome measures of physical and psychological premenstrual symptoms, depression, anxiety and anger. The outcome scores of Group I in these assessments were significantly lower than those of Group II. In the second scenario, there were significant differences between scores in all assessment tools including physical and psychological premenstrual symptoms, depression, anxiety, anger and perceived stress at the end of the placebo and herbal treatment in Group II favouring the Chinese herbal treatment.

Placebo control groups in clinical trials are essential to minimize bias, to allow for discrimination of effects caused by factors such as spontaneous changes in the disease, patient expectations or from other causes, and thus to allow a stronger interpretation of the result (Ossege et al, 2005). The presence of highly significant differences between placebo treatment and Chinese herbal treatment in the current study further provides strong evidence that the therapeutic efficacy of Chinese herbal medicine is considerably stronger than that of a 
placebo. Chinese herbal medicine is therefore considered to be an effective alternative treatment method for PMS symptoms.

\subsubsection{Hypothesis 3 , the changes in symptoms occurrence and severity of PMS will be maintained at three month follow-up.}

This was partially supported indicated by the observation of as significant differences between baseline and follow-up, with significant adverse differences between end of herbal treatment and follow-up.

Outcome scores of premenstrual physical and psychological symptoms in Groups II indicate that the herbal treatment effects at the three month follow up diminished in comparison to the effect at the last month of the herbal treatment period, but still maintained significant improvement over pre-treatment. These findings confirm that the reduction in symptoms occurrence and severity of PMS was maintained at three month follow-up. Also, the absence of any significant differences between end of treatment and one month after treatment suggests that the reduction in symptoms occurrence and severity at the end of treatment was maintained after one month.

Clinical significance tests further supported that the reduction in symptoms occurrence and severity of PMS was maintained at three month follow-up. The percent improvement remained greater than $35 \%$ which indicates a clinical significant difference (Long, 1995) as compared to conditions before the herbal 
treatment, and the effect sizes (ranged from .8 to 1.30) of these symptom groups remained within the large clinical effect (Cohen 1988) three months after the completion of the herbal treatment.

This finding of the present study indicates that there was still significant treatment effect both statistically and clinically from the Chinese herbal medicine three months after the treatment had ceased, which is consistent with other studies of Qiao and colleagues (2002); and Li and colleagues (2002) in which the herbal effect was maintained significantly at 3-6 months follow-up.

However, in the present study, the treatment effect was found to be reducing from one month after herbal treatment period. Also, significant adverse differences were observed between end of herbal treatment and follow-up. A possible explanation is that the herbal mixture, which was prepared by mixing individually prepared herbal granules together before dissolving them to facilitate ingestion, used in the current study may not have been strong enough to re-establish and maintain the full balance of yang and yin in some PMS sufferers. Consequently, certain symptoms reappeared after ceasation of herbal treatment. Guo (1996) and Wang and colleagues (2002) both have pointed out that mixtures or granules prepared from decocting all herbs together are more effective than those prepared from the mixing of individual herbs. This approach can be adopted readily in China but is not achievable in western countries like Australia where Chinese herbs suppliers will not provide medicine manufactured from decocted herbs unless for very large orders. Therefore for a small study like this one, the researcher was forced to rely on 
the more readily accessible and practical method available. Even so, a clinically effective outcome was demonstrated.

\subsubsection{Hypothesis 4 , the rating of client satisfaction with treatment will be positively related to the treatment outcome.}

This was supported as all correlations between the client's satisfaction rating and all clinical assessments including premenstrual physical and psychological symptoms, depression, anxiety, anger and stress were negatively related.

Donabedian (1982 cited in Turner \& Pol 1995) pointed out that client satisfaction gives information on the provider's success at meeting those client values and expectations and is of fundamental importance as a measure of the

quality of care. McLellan and Hunkeler (1998) suggested that client satisfaction is related to treatment outcome from the client's perspective. Client satisfaction is, after all, of paramount importance to any treatment effort.

This finding in the current study also suggests that there were highly significant associations between perceived efficacy of Chinese herbal treatment and patient satisfaction on PMS, and further supports the view that Chinese herbal medicine can reduce symptoms occurrence and severity of PMS, and can bring about client satisfaction with the herbal treatment they receive. 


\subsection{Adverse Effects of Herbs}

No adverse effect was anticipated as all the herbs used are commonly accepted and applied to a wide range of health problems, and are approved by the Therapeutic Goods Administration, Department of Health and Ageing, Australian Government. In the current study, no adverse effect was reported except that some women did not like the taste of the substances, whether they were Chinese herbs or placebo. Three participants withdrew from the study because they disliked the taste. When the code was broken it was found that two of these women were in fact in the placebo arm of the study. This undesirable taste that the participants are likely to experience is only temporary, and poses no long-term adverse effect to the participants. In fact, they all learnt to overcome this difficulty eventually by taking the granules with juice or mixing them with honey, jam or apple paste. No other adverse effect or discomfort was reported.

The Therapeutic Goods Administration (TGA), a unit of the Australian Government Department of Health and Ageing, carries out a range of assessment and monitoring activities to ensure therapeutic goods available in Australia are of an acceptable standard with the aim of ensuring that the Australian community has access, within a reasonable time, to therapeutic advances.

This finding strongly supports that Chinese herbs which are approved by the TGA are safe and can provide an ideal alternative treatment method to western 
drugs for PMS sufferers, many of them are having unwanted side-effects with their current treatment.

\subsection{Placebo Effect}

Placebo effects have been demonstrated in the participants in this study as in many other studies (e.g. Yonkers et al. 1997; Walsh \& Polus 1999; Freeman \& Rickels 1999; Steiner et al. 2003; Pearlstein et al. 2005a). At the end of the first month of the study, there was a fall in the outcome scores of all clinical assessments (premenstrual physical and psychological symptoms, depression, anxiety, anger and perceived stress) in both the Chinese herbal treatment group (Group I) and the placebo treatment group (Group II). The possible explanation to this generic treatment effect is the effect from placebo. The "placebo effect" was clearly apparent in all clinical assessments as reductions of scores ranging from $7.2 \%$ to $33.9 \%$, and significant treatment effects $(\mathrm{p} \leqq .001)$ were found on the constructed composite physical and psychological symptoms of MDQ (Menstrual Distress Questionnaire) in the women of the placebo group at this time stage. Although reductions in all clinical assessment scores in the Chinese herbal medicine group were larger than the placebo group, the treatment effects between the two groups were not statistically significant in any assessment outcome $(\mathrm{p}>.05)$ at this time. This finding is consistent with previous studies of PMS. A meta-analysis of controlled treatment trials for premenstrual dysphoric disorder showed rates of placebo response ranging from $6 \%$ to $35 \%$ (Yonkers et al. 1997). An explanation of placebo responses is proposed as 
being the expectation of clinical benefit or a conditioned response (Haour 2005) that overtime subsequently fails to be supported by an active agent. There have been no conclusions drawn about the mechanisms involved in the placebo effect (Stewart-Williams \& Podd 2004). However, recent new techniques of investigation in neuroscience suggest that there is a biochemical basis for the placebo effect and that it is the result of the activation of the limbic system of the brain. Dopamine, endorphins, seratonin, opioids and other small molecules have been shown to be mediators of placebo responses (de la Fuente-Fernandez \& Stoess1 2004).

From the second month onward, there was a change in Group II in the direction of the outcome scores of all clinical assessments. The scores either went up or remained the same which indicated a turning point that the placebo effect was fading out. However, by the end of the third month, the "placebo effect" was still observed in all clinical assessments as reductions of scores ranging from $5.1 \%$ to $20.7 \%$ and there were still significant differences in comparison to pre-treatment on psychological premenstrual symptoms and anxiety. This finding is consistent with the finding in Freeman and Rickels (1999) in which over $60 \%$ of placebo treated subjects showed partial or sustained improvement at the end of a three month double blinded PMS study. However, the significant differences between placebo and Chinese herbal treatment on all outcome scores at the end of the treatment in the current study confirm that the effect of Chinese herbal medicine significantly exceeds the placebo effect. 


\subsection{The TCM Patterns of Disharmony}

Participants of the current study were allocated into four groups based on their respective patterns of disharmony identified through the process of TCM Pattern Identification. Although some variations in herbal ingredients were necessary and applied for the different TCM pattern groups of the study, no significant statistical differences in treatment effect were noted among the various pattern groups in the three months of Chinese herbal treatment. This finding further supports Pattern Identification, the traditional diagnostic method of Chinese medicine which has been used in China since the Eastern Han Dynasty (25-220AD), as a valid tool for diagnosis and treatment application in clinical Chinese medicine.

Within the four patterns of disharmony in PMS in the current study, Pattern A, Liver $Q i$ Stagnation, achieved a greater treatment effect than the other patterns after three months of treatment with herbal granules. This is consistent with Zhu and Hu's (2003) finding that $70.71 \%$ of women with Liver Qi Stagnation in their study achieved outstanding treatment effects of herbal medicine. This finding supports the theory that Liver $Q i$ Stagnation is the primary cause of PMS, and that there is no involvement of other organs, simply only the Liver system is affected. In comparison with the other patterns where more organ-systems are involved, it is a lot easier and quicker to regain the balance of one organ-system in the body. 
One of the common TCM patterns in PMS i.e. Phlegm and Qi/Blood binding together causing obstruction did not appear in the current study. The likely explanation is that this pattern usually appear in long term PMS sufferers, when long term Liver $Q i$ stagnation transforms into Fire, the Fire scorches body fluids (Water and/or Dampness) into Phlegm. Women with this pattern always report more serious and manic-like symptoms since Phlegm and Qi/Blood binding together in the channels with the Fire exacerbates the symptoms. Most sufferers of the severe type of PMS in Australia are either prescribed antidepressants or other pharmacological medications which, in this study constituted an exclusion criterion.

\subsection{TCM Symptom Patterns and Western Perspective Symptom Patterns}

The four patterns of disharmony of TCM are grouped according to Patterns Identification which is based on TCM aetiological and pathogenetic theories. From a western perspective, Abraham (1983) grouped PMS symptoms according to their nutritional aetiological factors and their pathophysiologies into four subgroups of Premenstrual Tension Syndrome, namely PMT-A, PMT-H, PMT-C and PMT-D. (see Table 2.3).

Interestingly, when compared to Abraham's subgroups of PMT, there are many similarities between the TCM patterns of the present study and the PMT subgroups. PMT-A shares similar symptoms with Pattern A of the present study. Liver $Q i$ stagnation is the primary cause of PMS, and all TCM patterns 
include symptoms of Liver Qi Stagnation, whilst PMT-A was the most common subgroup in Goei and Abraham's (1983) study, in that over 90\% of their PMS sufferers showed symptoms of PMT-A. PMT-H and Pattern H of the present study share similar symptoms. PMT-H is associated with symptoms of water and salt retentions, whereas Liver and Spleen disharmony of Pattern $\mathrm{H}$ results in the failure of the Spleen to transform and transport fluids throughout the body properly, thus giving rise to Dampness retention. PMT-C shares similar symptoms with Pattern CA of the present study in which Liver and Kidney Yin Deficiency leads to heat in the internal organs. PMT-D has similar symptoms to Pattern D of the present study in that Heart and Spleen Deficiency results in the spirit and energy of the body being disturbed. PMT-A and PMT-H were the two most common subgroups in Goei and Abraham's study (1983), which correspond to Pattern A and $\mathrm{H}$ in the present study: where $29 \%$ of women expressed Pattern A symptomatology and 32\% reported symptoms according to Pattern H.

The findings indicate that these two studies, irrespective of the method of classification, i.e. whether it be a western nutritional aetiology or TCM approach using Pattern Identification, share a high degree of similarity in the classification of PMS. Future research in these complementary approaches may wish to investigate whether or not there are similar nutritional ingredients as suggested in the Abraham study (1983) and those contained in the Chinese herbs prescribed in this study for the treatment of PMS. 
The negative affective symptoms eg anxiety, irritability, depression, sadness, mood swing, agitation, manifested in Liver Qi Stagnation / PMT-A are the most frequently reported symptoms of PMS in these two studies. These results are consistent with previous studies in Australia and China. In Australia, Gott, Morse \& Dennerstein (1995) stated that the most frequently reported symptoms were irritability and depression among 98 PMS treatment seeking women in Melbourne, Australia. Kirkby (1994) reported that the symptoms rated most often as severe were irritability (62\% of sample), depression (56\%), tension (49\%), fatigue (36\%), loneliness (31\%), and decreased efficiency (31\%) among the 48 women with "serious premenstrual problems" of his study in Melbourne. Gao and Chen (1994) collected, collated and categorised the TCM symptom patterns in PMS from journal articles over a period of ten years. The most common patterns encountered were Liver $Q i$ Stagnation. The order of frequency of the PMS symptoms was irritability, depression, anxiety, diarrhoea, lack of concentration and hypersomnia in the Zhao and colleagues' study (1998) on Chinese women in Beijing.

\subsection{Signs and Symptoms on Tongue and Pulses}

Examination of the tongue and pulse are two important diagnostic methods particular to TCM. Long term clinical TCM practice suggests that any changes in the tongue and pulse reflect the physiological and pathological condition of Qi, Blood, body fluid and the organs in the body (Ding \& Sun 2003). According to TCM theory, tongue connects other organs with the help of 
meridians throughout the body, so the conditions of the internal environment are manifested externally by the tongue. Tongue coating is formed by the rising of the $Q i$ of the Spleen and Stomach, thus any transformation process in the body is exhibited and can be determined by the tongue coating. Pulse taking in modern TCM practice refers to the feeling of the pulse of the radial arteries at the styloid processus of both wrists, which is known as "Cun Kou" in TCM, with the practitioner's fingers (Flaws 1997). It is believed by the practitioners of TCM that Cun Kou is one of the major meeting points of the vessels/ meridians. Other than the speed, the strength, the depth and the quality of the pulse are measured by the practitioner, and different pulse symptoms indicate different physiological and pathological state of the body. However, one of the drawbacks in relying on pulse taking for diagnosis is that the determination of pulse symptom is a very subjective process. Two practitioners may feel and interpret a patient's pulse differently. A special device to detect the pulse and print out the result is being developed and under testing in China. The device is called sphygmogram which should aid in standardizing the measurement of pulse symptoms (Dharmananda 2000).

In the current study, about $70 \%$ of the PMS sufferers had a normal tongue which was pink with thin white coating. However, over $44 \%$ had teeth marks on their tongues indicative of Spleen deficiency or Dampness retention in the body (Zhang, 1990b). $41 \%$ had purplish spots or areas on the tongue which indicate stagnation of $Q i$ or Blood in the body. Over one third showed vertical cracks on their tongues indicative of deficiency of blood, Yin or Stomach Qi. More than a quarter had a swollen/enlarged tongue which is a sign of dampness 
retention in the body. $32.7 \%$ had a yellowish tongue coating which indicates a syndrome of interior Heat, and $18 \%$ women had a red or dark red tongue, a sign of Heat syndrome. After three months of herbal treatment, there was no more red or dark red tongue, only $6.6 \%$ of women's tongue coating remained yellowish, $8.2 \%$ of women still had purplish spots on their tongues, and swelling, teeth marks, and vertical cracks in their tongues had reduced by more than $40 \%$. These changes in tongue symptoms reflected that those predisposing factors of the disorder i.e. Heat, Dampness, Deficiencies, $Q i$ and Blood Stagnation were dramatically reduced within these women.

According to TCM theory, pulse symptoms reflect the state of $Q i$, Blood and the organs of the body. In the current study, the most common presenting pulses were wiry, thin, slippery and choppy. More than $70 \%$ of women in the study had a combination of wiry, thin and slippery pulses at the first interview. A wiry pulse indicates disorders in the Liver and Gall-Bladder systems and $Q i$ Stagnation. It reflects Liver $Q i$ stagnation which is the primary cause of Premenstrual syndrome. A thin pulse indicates a deficient syndrome including $Q i$, Blood, or yin fluid deficiency, or a chronic/prolonged disorder. The thin pulse of these women suggests that they have been suffering from the syndrome for a long period. A slippery pulse indicates Dampness or Phlegm retention in the body, but it is recognized as normal in women during menstruation or pregnancy when their blood/fluid volumes are increased (Luo, 1986). Since the pulse symptoms of these women were taken at the interviews during or soon after their menstruation, there was profuse fluid or dampness in their body. A choppy pulse indicates either stagnation of $Q i$, Blood, food or 
phlegm, or deficiency in $Q i$, Blood, Yin or body fluid. TCM believes that women are prone to $Q i$ and yin blood deficiency due to regular blood loses during menstruation and deliveries which explains the frequent appearance of choppy pulses in these women. After three months of herbal treatment, there were $60 \%, 50 \%$ and $40 \%$ reduction in slippery, choppy and thin pulses respectively which indicate the treatment efficacy of herbal medicine. However, $80 \%$ of women still demonstrated a wiry pulse after treatment. The possible explanation is that either it is a natural reflection of the physiological changes in the body during premenstrual period as blood and $Q i$ travel down to the uterus via the meridians for the preparation of menstruation thus causing blockage of the meridians, or the herbal treatment for only three months was not long enough or the herbal mixtures that had been produced by mixing individually prepared herbal granules together was insufficient in strength to restore the full balance in the Liver systems of the PMS sufferers. A stronger and/or larger dosage commensurate to the bigger western body build and/or individual PMS severity should be explored.

The tongue signs and pulse symptoms of these women corresponded to their TCM patterns (See Table 7.1). In pattern A, Liver $Q i$ stagnation, most women demonstrated a normal pink tongue with thin white coating, and wiry and slippery/thin pulses. In pattern CA, Liver and Kidney Yin deficiency causing heat in the internal organs, over $80 \%$ of women demonstrated a red or pink tongue with thin and yellowish coating, and wiry and thin pulses. In Pattern D, Heart \& Spleen deficiency, 75\% of women demonstrated a pale tongue with white, thin or moderate thickness coating, and $91 \%$ of women had a thin pulse. 
In pattern $\mathrm{H}$, Spleen deficiency arising from Liver \& Spleen disharmony causing Dampness retention, $86 \%$ of women demonstrated a pale or pink tongue, $38 \%$ had a swollen or enlarged tongue, $71 \%$ had teeth marks on their tongues, and over $75 \%$ of women had wiry and slippery pulses. Usually, women with Spleen deficiency causing Dampness retention show symptoms mainly related to Water retention and stagnations which are less likely to occur. Interestingly, women in this group had a higher percentage $(42.9 \%)$ of purplish spots/areas, which indicated stagnation on their tongues, than other pattern groups. However, the above finding may not be compared with other PMS studies of TCM since no information on the analysis and changes of TCM tongue signs and pulse symptoms is available from TCM literature and web search.

\subsection{Sample characteristics}

The personal profile of the women in the current study showed a similarity in menstrual history to those in previous studies (Morse et al. 1991; Kirkby 1994; Campbell et al. 1997; Kirkby \& Lindner 1998; Walsh \& Polus 1999) on PMS among Australian women. This profile revealed that the median menarche is around 13 years of age, length of cycle is 28 days and length of menses is 5 days. However, there were some differences. The mean age of women in the present study was 31.8 years (ranging from 19-42), which was relatively younger than the women in the studies of Morse and colleagues (1991) and Walsh \& Polus (1999) that had a mean age of around 35. Kirkby (1994), 
however, reported a mean age of 28.9 (ranging 18-47) of those women in his study. Only $4.6 \%$ of the women in the present study reported that they were homemakers, in comparison to 25\% in 1997 (Campbell and colleagues) and $33 \%$ in 1991(Morse et al.). This finding may reflect the changes in Australian social trends. Australian Bureau of Statistics (ABS 2005) stated that around $70 \%$ of females aged between $25-44$ years participate in the labour force in the years $2003-4$ in comparison to $54 \%$ of those aged $25-34$ and $58 \%$ of aged $35-44$ in 1983-84. Just under $42 \%$ of the women in the present study were married as opposed to 90\% in Morse and colleagues' study (1991) and 62\% in Campbell and colleagues' study (1997). ABS (2001) stated that 50.3\% of Australian women were married, and $12 \%$ of all persons living as socially married were de facto partners in 2001. Unfortunately, the researcher did not categorize the marital status of "married" in detail which makes it impossible to identify marital status more finely. Women in this study were highly educated. 52.5\% were university graduates and $21 \%$ had post secondary training, whereas only $18 \%$ of women had a college education in Campbell and colleagues' study (1997). Previous research revealed that premenstrual symptoms were more severe among women engaged in professional studies (Marvan et al. 1998); career women had more premenstrual symptoms than homemakers, and marital stress contributed to the severity of PMS symptoms (Coughlin 1990). In contrast, Hsiao (2004) and colleagues reported that no relationship was found between premenstrual symptoms and years of education or marital status. These social changes may have a bearing on the occurrence and severity of PMS among women in the present society of Australia. However, further investigation of this aspect is outside the scope of this study. 
PMS experiences

In Morse and colleagues' (1991) study, carried out in the same metropolitan city as the present study, $85 \%$ of women reported symptom changes during the premenstrual period causing serious or severe adverse effects and only $15 \%$ of women reported moderate effects. Conversely, in the study of Yonkers and colleagues (2003), 35\% of women who endorsed current PMS symptoms identified their premenstrual symptoms as a "serious problem". Women in Morse and colleagues' (1991) study had a median of 10 days symptom onset and $44 \%$ of the sample reported symptom relief by the end of menstruation (around Day3-5). In the present study, symptom changes causing serious or severe adverse effects on life were reported in $41.6 \%$ of the women with over half $(50.8 \%)$ of the women reporting moderate adverse effects, and a median of 9 days of the total days of PMS experiences per cycle. Thus, the women in the present study may be considered as a medium severity group of PMS sufferers.

The women in the present study were troubled with psychological symptoms i.e. anxiety, anger and depression, and their pre-treatment scores of premenstrual physical and psychological symptoms were higher than normal on the MDQ in all symptom clusters except arousal which was in line with the norm. These findings are consistent with previous PMS studies (Moos 1985; Morse et al. 1991; Pearlstein et al. 2005b). However, the mean perceived stress of these women fell within one standard deviation from the normative group and as such cannot be viewed as indicating a stress effect. This finding is in contrast to 
other studies (Woods et al. 1998; Hylan et al. 1999; Lustyk et al. 2004) that found women with PMS reporting higher stress levels.

The leading presenting symptoms of this group of women were irritability, mood swings, fatigue, anxiety, breast tenderness, depression, tension, and swelling. These findings are very similar to the reports in studies in China (Zhao et al. 1998), Hong Kong (Holroyd et al. 1998), United States, United Kingdom and France (Hylan et al. 1999). (see Subdivision 2.5).

\subsection{Menstrual Characteristics}

Most women of the present study reported that they had regular menses with normal blood loss, colour and nature. However, the majority (98.5\%) reported that there were clots in their menses, $55.4 \%$ of women reported large pieces of clots and $43.1 \%$ reported small clots. In Chinese medicine, small pieces of clots in the menstrual bleeding are usually associated with $Q i$ and Blood Stagnation, whilst large clots are associated with Blood Stasis (Lo 1994). Since the Liver system is the regulator of $Q i$, it ensures the smooth flow of $Q i$ throughout the body, and $Q i$ is recognized as the motive force of the blood. When $Q i$ is not moving smoothly in the body, blood will also circulate sluggishly which is known as $Q i$ and Blood Stagnation in TCM. Long term $Q i$ and Blood stagnation may eventually lead to Blood stasis (Maciocia 1989). The excess of clots in PMS women of the present study was in accord with the main etiology of PMS in TCM which is Liver Qi Stagnation. 


\subsection{Individual Symptoms Ratings}

The most common presenting premenstrual symptoms in the MDQ before treatment in women $(n=65)$ of the current study were irritability $(96.9 \%)$, mood swings (96.9\%), fatigue (95.4\%), anxiety (90.8\%), depression (89.2\%), tension (89.2\%) and swelling (87.7\%). Most of these symptoms are "Negative Affect" items as measured by the MDQ (Moos 1969). This finding is consistent with previous studies in China (Gao and Chen 1994; You 1997; Ran 1997; Wang X et al. 2003) that Liver $Q i$ stagnation is the most prevalent TCM pattern of PMS and further supports the TCM theory that Liver $Q i$ stagnation is the primary cause of PMS. Since TCM proposes that one of the regulating functions of the Liver system is to smooth and regulate the mind and mood of human beings, when the Liver $Q i$ is obstructed and cannot move freely, the mind and mood are disturbed, and mental and emotional symptoms appear (Maciocia 1989).

The results recorded on the MDQ in the current study indicate that women receiving herbal treatment reported highly significant reduction in severity and occurrence of most symptoms including both psychological and physical symptoms. The findings of the daily diaries (MSD) of a reduced number of participants show very similar results to those from the MDQ. The best results of herbal treatment effect were shown in the following premenstrual symptoms: anxiety, irritability, fatigue, breasts tenderness, mood swings, nervous tension, swelling and depression. However, there was no significant treatment effect for craving of sweet, heart pounding and forgetfulness in the MSD. The possible 
explanation to these results is that palpitation is a common symptom for any woman. The analysis of symptom ratings in the MSD of the current study did not include a comparison between luteal phase and follicular phase on each of the symptoms, so it is possible that heart pounding was not associated with PMS. In TCM, craving of sweet is related to Spleen deficiency, forgetfulness is related to Heart and Spleen deficiency and heart pounding is related to Heart deficiency. These symptoms are deficient in natures which usually require longer treatment time to achieve a full balance.

\subsection{Prospective and Retrospective Reports of Symptoms}

Prospective record keeping has been claimed to be rather time-consuming for participants (van der Ploeg \& Lodder 1993) that leads to high drop-out rates in studies (Haywood et al. 2002). Alternatively, utilising recall of symptoms and their severity in retrospective reports has been criticized by researchers as less accurate, as it may be influenced by subject recall bias (Woods et al. 1982a).

From the outset of the current study, the women were asked to fill the menstrual diary prospectively, and the MDQ, STPI, BDI and PPS retrospectively every month for the duration of the study. During the assessment stage, the assessments were based on both prospective (to meet the requirement of ACOG for the diagnosis of PMS) and retrospective reports (as the basic information). Only those who had completed all the questionnaires and the menstrual dairies were invited to take part in the study. Failure to 
record the symptoms everyday was the main reason for a $54 \%$ attrition of participants during that stage which gave rise to a second round of recruitment of subjects, and an extension of the study. Furthermore, the same problem happened in the treatment period as most women failed to return a complete menstrual dairy for some months. Some had a few days' information missing, and some had even lost their menstrual diaries. Only 29 (47.5\%) women charted their menstrual dairies completely in their premenstrual phases during the entire herbal treatment periods. This large percentage of missing data, i.e. only the data of 17 women in Group I and 12 women in Group II could be used, and might give rise to unreliable results when the statistics of individual symptoms between groups are analysed. The finding of the current study that prospective records lead to high drop-out rates is consistent with those of previous studies (Richter et al. 1984; Gise et al. 1990; Sveindottir and Backstrom 2000).

Analysis of the current study indicates no difference in all assessment scores at baseline except depression between those women who were able to record their premenstrual symptoms on the menstrual diaries continuously and who were those not. Women who failed to complete charting their premenstrual symptoms in all the menstrual diaries in the study showed higher scores on the depression questionnaire (BDI) than those who completed the diaries. Future studies may wish to take into account the apparent trait and particular circumstances of each candidate with a view to predicting which type of women will have difficulty in recording symptoms on a daily basis, and whether severe depression is the predisposing cause of failing to keep records continuously. 
The results of the analysis of herbal treatment effects on PMS from the data of the MDQ which were collected retrospectively were very similar to the results from the MSD which were collected prospectively in the current study. The results were also consistent with previous studies (Kim 2004; Steiners et al. 2005). Kim (2004) stated that highly rated symptoms on the prospective and retrospective reports were found to be similar, whilst Steiners and colleagues (2005) pointed out that the results did not differ regardless of whether the premenstrual data were collected prospectively or retrospectively in their study.

\subsection{Alcohol and smoking}

In the present study, $26.1 \%$ of women consumed alcohol daily which was higher than the average percentage $(6 \%)$ of Australian women aged 14 years and over in 2002 (Australian Institute of Health and Welfare). The result was consistent with previous findings. Studies (Perry et al 2004, Chuong \& Burgos 1995; Caan 1993) suggest that higher alcohol consumption has been associated with increased premenstrual symptoms. However, none of the women in the study consumed in excess of the low risk level in the Australia Alcohol Guidelines (NHMRC 2001) i.e. up to 2 standard drinks per day.

Similarly, the results of some of the prior studies (Kritz-Silverstein et al. 1999; Mishra 2000) show that smokers have an increased risk of menstrual-related problems. In the present study, the finding that only $13.8 \%$ of the participants 
were smokers was inconsistent with the findings of other studies such as in Strine and colleagues' study (2005) in that $27.8 \%$ of PMS women were smokers, and in Masho and colleagues' study (2005) where $43 \%$ of the subjects were smokers. The percentage of women who were smokers in the current study was also lower than the average percentage (21\%) of Australian women in the National Health Survey 2001 (ABS, 2001).

\subsection{Ethnicity and Treatment Efficacy}

The range of different ethnicities was recoded into two groups. Women of Group A ( $n=37)$ were Australasian or British who have an English speaking background, and women in Group B $(n=24)$ were European or Asian where English was really their second language although they experienced no difficulties in reading, writing and speaking Standard English.

There were no significant differences in the scores of any clinical assessment or time phase for the two ethnic groups. That is to say the efficacy of Chinese herbal medicine is not influenced by the different ethnic backgrounds of the women, be it Australasian, British, European or Asian. It follows that treatment of PMS with Chinese herbs should be an ideal alternative treatment option acceptable to Australian women of any ethnicity.

Since there were only 5 Asian women among the 61 participants, it was not possible to estimate any difference between women with Asian and Western 
backgrounds. And in Group B, less than one quarter of women were Asian, and most of them were of western origin similar to those in Group A. The homogenous nature in ethnicity of these women can be an alternative explanation for the result.

\subsection{Subject Attrition}

During the two-month assessment period, there were 82 withdrawals out of a total of 152 participants giving a drop-out rate of $54 \%$. High attrition has been a common problem for some previous studies (Gise et al 1990; Miller et al 1999; Sveindottir and Backstrom 2000). Once treatment had commenced, only four women withdrew $(6.2 \%)$ during the entire treatment period - one due to pregnancy and the other three did not like the taste of the substances (Chinese herbs or placebo).

From telephone enquiries, the most common reason for withdrawal in the current study was failure to complete the menstrual dairies due to forgetfulness (further details in subdivision 7.2.1). Haspels (1981) also reported that the most frequent given reason from those who withdrew was difficulty to comply with the request to record symptoms each day. It is a requirement of the American College of Obstetricians and Gynaecologists (ACOG) and the American Psychological Association (APA) that information for the diagnosis of PMS should be based on the daily diaries kept by women charting their symptoms, preferably for two to three consecutive months. This is a very demanding task 
which is, nevertheless, necessary when strict protocols must be followed in research. The successful completion requires a very high commitment by the women concerned. This willingness to adhere to an onerous demand must render this to be somewhat atypical. The National Institute of Mental Health also suggests that the symptoms of PMS have to be present during at least two consecutive cycles. Further investigation should look for an easier and less complicated way to diagnose PMS.

\subsection{Limitations}

There are several limitations to the present study. First, the washout period of Chinese herbs was unknown as no laboratory reports can be found and some studies in China reported that treatment effects of their herbal medicines can continue up to 3-12 months (Lu 2001; Qiao 2002; Zhang 2003b). Thus the within subjects effects in Group I were not compared, and the reliability of the within subject effect in Group I of the crossover design is weakened. Consequently, the researcher mistakenly did not collect data from women of Group I at follow-up, leading to failure of comparison of the treatment effect between groups at follow-up. Second, the data were based on self-reports which were not validated by laboratory or medical examinations. There is a possibility that the reported information may be under or over estimated. The self perceptions of the normality or abnormality on menstrual characteristics might differ for each participant resulting from different personal experiences and family background. Third, due to the unavailability of herbal granule which 
has to be prepared from decocting all herbal ingredients together for this study, the effective rate of the herbal treatment on PMS may have been reduced considerably. Finally, data of personality and demographics were not appraised before the assessment period. It was impossible to evaluate the personality and the demographic profile of the withdrawals that may provide further reasons for high drop-out rates in studies.

\subsection{Summary}

In summary, analysis of data of this study identified and pinpointed the negative symptoms manifested in Liver $Q i$ Stagnation as being the most frequently reported PMS symptoms. Hypotheses 1-4 were supported both statistically and clinically which further reinforces the case for Chinese herbal medicine to be used because of their effectiveness and safeness for the treatment of PMS irrespective of the social, physical and ethnical background of the patient. Pattern Identification, tongue signs and pulse symptoms have been proved to be reliable tools of the diagnosis of PMS in Chinese medicine. Participants of this research project showed a fair degree of similarity to those of previous PMS studies in terms of social and physiological characteristics with some exceptions in such areas as educational background and stress levels. It is considered that the finding of this study will contribute in some ways towards:-

- the demonstrated effectiveness of Chinese herbal medicine for PMS symptoms; 
- further development of evidence-based scientific research in TCM;

- offering an alternative treatment option to western drugs for PMS sufferers;

and

- $\quad$ supporting/vindicating the results of many previous TCM studies on PMS

in China (such as Dou 2004; Ma 2004; Zhang 2004a; Chen 2001; Ye 1998;

Liang 1996). These researches used pattern identification to diagnose the disorder but did not follow a scientific research approach, or robust

methods by which these studies were carried out. Therefore, replication, one of the hallmarks of the scientific method, has not been possible. 
Chapter 12: Conclusion, Recommendations and Future Directions

Page

12.1 Conclusion

292

12.2 Recommendations on the TCM Treatment of PMS

293

12.3 Future Directions on PMS Studies of Chinese Medicine 


\subsection{Conclusion}

The research findings for the treatment of PMS sufferers in a western society reported here clearly demonstrate that there is an effective alternative treatment option, namely Chinese herbal medicine.

The results of the study indicate that Chinese herbal medicine can lower the symptoms occurrence and severity of PMS significantly, and the effect of the treatment can last up to three months after herbal treatment has ceased. Even though large placebo effects have been reported in PMS studies utilizing a wide range of pharmaceutical agents, the results of this study demonstrates significant differences between the treatment effects of herbal medicine and placebo treatment favouring the herbs. It is also evident that this treatment can bring about client satisfaction with the herbal medicine they receive. There was no adverse effect reported during the whole course of the study which suggests that the Chinese herbal medicine is an ideal alternative treatment method to western drugs for PMS sufferers, many of them are having unwanted side-effects with their current treatment.

The review of TCM clinical trials in Chapter 5 of this thesis has confirmed the perception held by many that most of the research in PMS performed in China using Chinese herbs was not scientifically evaluated utilizing strong research designs. The current study was carried out with a robust western scientific approach, that is, a double-blind controlled clinical trial, and the application of herbal treatment was based on Pattern Identification, a traditional method that 
has been used by TCM professionals for hundreds of years. The findings of this study provide new evidence based information using Chinese herbal medicine for the treatment of PMS and in a way supports previous TCM research which did not use a scientific research approach nor a rigorous evaluation method (Chou \& Morse, 2005).

Great emphasis was placed in this study on the analysis of pulse symptoms and tongue signs of PMS according to TCM tradition, which have proved to be valuable and reliable tools to aid diagnosis and to gauge the effectiveness of herbal medicine for the treatment of PMS. This has also opened up a new area for further TCM research. The findings should also provide the necessary statistical data to further support the use of Chinese herbs for the treatment of PMS and should contribute in some measure to the development of an evidence-based scientific research in traditional Chinese medicine.

\subsection{Recommendations on the TCM Treatment of PMS}

Findings from the present study indicate that most participants showed signs and symptoms of Liver $Q i$ stagnation. This reinforces and coincides with the proposed theory of TCM that Liver $Q i$ stagnation is the primary cause of PMS (Cai 1997; Huang 1999; Wang 2000; Tian et al. 2003; Bi 2004; Zhang 2005; Tang 2005). Thus, a primary treatment strategy in TCM for PMS should be directed towards the Liver system, supplemented by other treatment applications depending on the TCM patterns of individual patients. This 
approach focuses on both the primary and secondary causes of the disorder, adhering to the foundational theory and principals of Pattern Identification in TCM as well as being able to deal with the idiosyncratic nature of PMS.

Whilst different herbs and formulas were prescribed for the various patterns of disorder, the basic medication used in this study was the modified Xiao Yao San. Additional herbs/formulas were added to the formula according to different TCM patterns. This strategy has proved to be very effective for the treatment of PMS in the study. Xiao Yao San is a classic formula with actions of coursing the Liver $Q i$, strengthening the Spleen and tonifying Blood. It has been commonly use by TCM practitioners for over seven hundred years with no adverse effects.

Since Chinese herbal medicine usually requires a longer period of time for its full treatment potential to take effect, it is prudent to advise patients undergoing treatment with Chinese herbal medicine for severe symptoms of PMS to adopt a combined approach with other medical professionals (e.g. western medical practitioners, psychologists, acupuncturist) until the full action of Chinese herbal medicine takes place. For patients experiencing acute pain, another option is to apply acupuncture to free the obstruction in the channels to alleviate pain e.g. breasts pain, headaches, and cramps. Patients with severe premenstrual dysphoric symptoms e.g. PMDD, may combine Chinese herbal medicine treatment for the first couple of cycles with western pharmacotherapy such as antidepressants for immediate relief. Thereafter, pharmacotherapy should be withdrawn to avoid side effects, and Chinese herbal medicine 
continued. As for patients with multiple psychological symptoms, cognitive behavioural coping therapy provided by a psychologist can be a great value in managing their psychological stresses. (see Morse et al. 1991; Kirkby, 1994; Blake et al., 1998; Goodale et al., 1990; Hunter et al. 2002)

\subsection{Future Directions on PMS Studies of Chinese Medicine}

Further work is clearly required to evaluate the contributions of Chinese herbal medicine for PMS using scientific methods. Firstly, every effort should be made to undertake more properly designed random-controlled trials on TCM in general and PMS in particular with a view to promoting TCM and its proper recognition by the western scientific world. Secondly, long term follow-up on the herbal treatment effects in subjects is necessary to evaluate the after effects of herbs and to decide on the appropriate length of time of treatment required for various symptom and severity. Thirdly, more data on TCM patterns, tongue signs and pulse symptoms are required to establish a set of standard TCM diagnostic tools for PMS and other disorders. Fourthly, sub-components of the trial could be evaluated to assess the efficacy of the herbal effect, such as the optimal minimal dosage of herbal medicine in symptom severity according to body build, format of the herbal medicine (raw herbs vs. granules), and time of treatment (how many days before menstruation). Lastly, consideration should be given for the combined application of western and Chinese medicine so as to make full use of their complimentary effects in order to achieve the best 
treatment outcome. More robust experimental designed studies on combined treatment therapy in PMS should be actively pursued. 


\section{References}

Abraham, GE 1980a, 'Premenstrual tension', Current Problem in Obstetrics \& Gynaecology, vol. 3, no. 12, pp. 1-39.

Abraham, GE 1980b, 'Premenstrual tension syndrome', Contemp Obstet Gynecol Nursing, vol. 3, pp. 170-84.

Abraham, GE 1983, 'Nutritional factors in the etiology of the premenstrual tension syndromes', J Reprod Med, vol.28, no.7, pp.446-64.

ACOG Practice Bulletin 2000, 'New PMS guidelines released: recommendations focus on diagnosis and treatment', AWHONN Lifelines, vol. 4, no. 3, pp. 61-62.

American College of Obstetricians and Gynecologists (ACOG) 2000, Premenstrual Syndrome, ACOG, Washington, DC.

American Psychiatric Association (APA) 1987, Diagnostic and statistical manual of mental disorders: DSM-IIIR, 3rd edn rev, American Psychiatric Association, Washington, D.C.

American Psychiatric Association 1994, Diagnostic and statistical manual of mental disorders: DSM-IV, 4th ed., American Psychiatric Association, Washington, DC.

American Psychiatric Association 2000, Diagnostic and statistical manual of mental disorders: DSM-IV-TR, 4th ed., Text revision, American Psychiatric Association, Washington, DC.

An, Y, Mao, D \& Wei, A 2005, 'Treatment of Premenstrual Syndrome by Acupuncture plus Psychotherapy’ (针刺配合心理疗法治疗经前期紧张综合 征), Shanghai Journal of Acupuncture and Moxibustion, vol. 24, no. 8, pp. 18-20.

Andersch, B, Svensson, A \& Hansson, L 1990, 'Pre-eclampsia, hypertension and the premenstrual tension syndrome', International journal of gynaecology and obstetrics (Sweden), Vol. 32, no. 2, pp. 123-7. 
Andersch, B, Wenderstam, C \& Hahn, L 1986, 'Prevalence of premenstrual symptoms in a Swedish urban population' J Psychosom Obstet \& Gynaecol, vol. 5, pp.35-49.

APA (American Psychiatric Association ) 1994, Diagnostic and statistical manual of mental disorders: DSM-IV, 4th edn, American Psychiatric Association, Washington, D.C.

APA (American Psychiatric Association) 1987, Diagnostic and statistical manual of mental disorders: DSM-IIIR, 3rd rev edn, American Psychiatric Association Washington, D.C.

Ashby, CR Jr, Carr, LA, Cook, CL, Steptoe, MM \& Franks, DD 1988, 'Alteration of platelet serotonergic mechanisms and monoamine oxidase activity on premenstrual syndrome', Biol. Psychiatry, vol. 24, pp. 225-33.

Atmaca, M, Kumru S \& Tezcan, E 2003, 'Fluoxetine versus Vitex agnus castus extract in the treatment of premenstrual dysphoric disorder', Human Psychopharmacology, vol. 18, no. 3, pp. 191-5.

Australian Bureau of Statistics (ABS) 2005 National Health Survey, ABS, Canberra.

Australian Bureau of Statistics (ABS) 2001 National Health Survey, ABS, Canberra.

Australian Institute of Health and Welfare (AIHW), 2004, A guide to Australian alcohol data, AIHW Cat.No. PHE 52, AIHW, Canberra

Author unknown. approx.100BC, Yellow Emperor's Classic of Internal Medicine (黄帝内经), Re-print 1997, Liaoning Science and Technology Press, China.

Backstrom, T \& Hammarback, S 1991, 'Premenstrual syndrome--psychiatric or gynaecological disorder?', Ann Med, vol. 23, no. 6, pp. 625-33.

Beck, AT \& Beck, RW 1972, 'Screening depressed patients in family practice. A rapid technic', Postgraduate medicine, vol. 52, no. 6, pp. 81-5. 
Becker, LA 1997, Statistical and Clinical Significance, UCCS, USA, viewed 1 March 2005, < Http://web.uccs.edu/lbecker/>.

Berger, CP \& Presser, B 1994, 'Alprazolam in the treatment of two subsamples of patients with late luteal phase dysphoric disorder', Obstet Gynecol, vol. 84, pp. 379-85.

Berke, ML, Blumer, A \& Zack, RG 2001, 'Treating Women with Menstrual Distress', Premenstrual Institute, viewed 3 March 2006, $<$ http://www.pmsinst.com>.

Bhatia, SC \& Bhatia, SK 2002, 'Diagnosis and treatment of premenstrual dysphoric disorder', American Family Physician, vol. 66, no. 7, pp. 1239-48.

Bi, H 2004, Treating 50 cases of premenstrual breasts pain by self designed Shu Gan Tong Luo Tang' (自拟疏肝通络汤治疗经前乳痛症 50 例), Zhejiang Journal of Traditional Chinese Medicin, 2004 Jan, p.23.

Blake, F, Salkovskis, P, Gath, D, Day, A \& Garrod, A 1998, 'Cognitive therapy for premenstrual syndrome:A controlled trial', J. Psychosom. Res, vol. 45, pp. 307-318.

Blumenthal, M, Klein, J, Gruenwald, J, Hall, T, Busse, WR \& Goldberg, A (eds) 1998, The Complete German Commission E Monographs: Therapeutic Guide to Herbal Medicines, Integrative Medicine Communications, Boston, MA.

Borenstein, M, Rothstein, H \& Cohen, J 2000, Power And Precision, Version 2, viewed 4 June 2001, <http://www.power-analysis.com/>

Brayshaw, ND \& Brayshaw, DD 1986, 'Thyroid hypofunction in premenstrual syndrome', The New England Journal Of Medicine, vol. 315, no. 23 , pp. 1486-7.

Brown, DJ 1994, 'Herbal research review: Vitex agnus castus clinical monograph', Quarterly Review of Natural Medicine, Summer, pp. 111-121.

Brush, MG, Bennett, T \& Hansen, K 1988, 'Pyridoxine in the treatment of premenstrual syndrome: a retrospective survey in 630 patients', Br J Clin Pract, vol. 42, pp. 448-52. 
Budeiri, DJ, Li, AWP \& Dornan, JC 1994, 'Clinical trials of treatments of premenstrual syndrome: entry criteria and scales for measuring treatment outcomes', British Journal of obstetrics and Gynecology, vol.101, pp.689-95.

Budeiri, D, Li, AWP \& Doman, JC 1996, 'Is evening primrose oil of value in the treatment of premenstrual syndrome?', Control Clin Trials, vol. 17, no. 1, pp. 60-8.

Caan, B, Duncan, D, Hiatt, R, Lewis, J, Chapman, J \& Armstrong, MA 1993, 'Association between alcoholic and caffeinated beverages and premenstrual syndrome', The Journal of reproductive medicine, vol. 38, no. 8, pp. 630-6.

Cai, H 1997, 'Observation of treatment of premenstrual syndrome by the method of coursing liver in 36 cases' (疏肝法治疗经前期综合征 38 例观察), Journal of Practical Traditional Chinese Medicine, vol. 13, no. 5, pp. 6-7.

Cai, J \& Wang, Y 2005, 'Treatment of 50 cases of premenstrual headache by modified Xiong Zhi Shi Gao Tang' (芦芷石膏汤加减治疗经行头痛50例), New Journal of Traditional Chinese, vol. 37, no. 3, pp. 75-6.

Cai, Z \& Zhou, P (eds.) 1997, Cai's clinical experience on gynecology (蔡氏 女科经验选集), Publishing House of Shanghai College of TCM, Shanghai, China.

Campbell, EM, Peterkin, D, O’Grady, K \& Sanson-Fisher, R 1997, 'Premenstrual symptoms in general practice patients. Prevalence and treatment', Journal of Reproductive Medicine vol. 42, no. 10, pp. 637-646.

Cao, N \& Qin, Z 2001, 'Clinical observation of treatment of premenstrual headache by Chuan Xiong Tang in 105 cases’ (川芦汤治疗经前头痛 105 例 临床观察), Journal of Shaanxi College of Traditional Chinese Medicine, vol. 24 , no. 5 , p. 40.

Cao, Y, Yuan, Y \& Zuo, R 2000, 'Treatment of premenstrual tension syndrome by integrated Chinese and western medicine' (中西医结合治疗经 前期紧张综合征), Journal of Yunnan College of Chinese Medicine, vol. 23, no. 3, pp. 54-5. 
Casper, RF \& Hearn, MT 1990, 'The effect of hysterectomy and bilateral oophorectomy in women with severe premenstrual syndrome', Am J Obstet Gynecol, vol. 162, no. 1, pp. 105-9.

Chang, H \& But, PP (eds.) 1996, Pharmacology and Applications of Chinese Materia Medica Vol I \& II, World Scientific, New Jersey.

Chau, JPC \& Chang, AM 1999, 'Effects of an educational programme on adolescents with premenstrual syndrome', Health Edu Res, vol. 14, no. 6, pp. 817-30.

Chen, B \& Chai, L 2001, 'Experience in the clinical treatment of premenstrual tension syndrome' (经前期紧张综合征临床治疗体会), Heilongjiang Journal of Traditional Chinese Medicine, no. 3, p. 34.

Chen, G \& Yang, S (eds.) 1991, Practical Diagnosis and Treatment-Integration of Western and Chinese Medicine (实用中西医结合 诊断治疗学), Traditional Medical Science and Technology Press, Beijing.

Chen, J \& Chen, D 2003, 'Treatment of 127 cases of premenstrual insomnia by Xue Fu Zhu Yu Tang' (血府逐瘀汤治疗经前失眠症 127 例), Shaanxi Journal of Traditional Chinese Medicine, vol. 24, no. 5, pp. 422-3.

Chen, L 1998, 'Fundamental knowledge of Chinese medicine on premenstrual tension syndrome' (经前期紧张综合征中医浅识), Journal of Shandong University of Traditional Chinese Medicine, vol. 22, no. 4, pp. 262-3.

Chen, L \& Zhao, D 1998, 'The application of liver coursing and regulating method on common gynaecological diseases' (疏肝调肝法在常见妇科疾病 中的应用), Capital Medicine, vol. 5, no. 1.

Chen, M 2005, 'Treating 21 cases of premenstrual syndrome by modified Dan Zhi Xiao Yao San' (丹枙逍遥散加减治疗经前期综合征21例), Journal of Practical Traditional Chinese Medicine, vol. 21, no. 2, p. 89.

Chen, S, Cheng, D, Guo, L, Sun, S \& Li, Y 2003, 'Treatment of 52 cases of premenstrual tension syndrome by Xue Fu Zhu Yu capsule' (血府逐瘀胶囊 治疗经前期紧张综合征52例), Beijing Journal of TCM, vol. 22, no. 6, p. 60. 
Chen, W (ed.) Ming Dynasty (1368-1644), The Supplementary and Explanatory Notes of Chen Su-an's Gynecology (陈素庵妇科补解), China.

Chen, W 2001, 'Observation on the treatment of 188 cases of premenstrual syndrome by pattern identification’ (辨证治疗经前期综合征 188 例观察), Journal of Practical Traditional Chinese Medicine, vol. 17, no. 1, p. 4-5.

Chen X, 2000, 'Experience in the treatment of premenstrual syndrome' (经 前期综合征治验), Shaanxi Journal of Traditional Chinese Medicine, vol. 21, no. 5 , pp. 217-8.

Chen, Y 1850, The Excerpt of Chen's Obstetrics and Gynecology (沈氏女科 辑要), China.

Chen, Z \& Chen, J 1996, 'Treatment of 30 cases of premenstrual mania and depression by liver coursing, stagnation resolving, blood moving and stasis transforming method' (舒肝解郁活血化疼法治疗经前期躁狂抑郁症 30 例), New Journal of Traditional Chinese, vol. 28, no. 3, p. 26.

Cheng, J, Xie, X \& Gao, G 1982, Treatment of 102 cases of premenstrual tension syndrome by TCM cycle therapy, (中医周期疗法治疗经前期紧张 征 102 例), Liaoning Journal of Traditional Chinese Medicine, no. 6, pp. 26-8.

Chou, PB \& Morse, CA 2005, 'Understanding premenstrual syndrome from a Chinese medicine perspective', J Altern Complement Med, vol. 11, no. 2, pp. 355-61.

Cohen, J 1988, Statistic power analysis for the behavioral sciences, $2^{\text {nd }}$ edn, Lawrence Earlbaum Associates, Hillsdale, NJ.

Cohen, S, Kamarck, T \& Mermelstein, R 1983, 'A global measure of perceived stress', Journal of Health and Social Behavior, vol. 24, pp. 385-96.

Condon, JT 1993, 'The premenstrual syndrome: a twin study', $B r \mathrm{~J}$ Psychiatry, vol. 162, pp. 481-486.

Christensen, AP \& Oei, TP 1995, 'The efficacy of cognitive behaviour therapy in treating premenstrual dysphoric changes', J. Affect. Disor, vol. 33, pp. 57-63. 
Chuong, CJ \& Burgos, DM 1995, 'Medical history in women with premenstrual syndrome', Journal of psychosomatic obstetrics and gynaecology, vol. 16, no. 1, pp. 21-7.

Coughlin, PC 1990, 'Premenstrual syndrome: how marital satisfaction and role choice affect symptom severity', The Social worker, vol. 35, no. 4, pp. $351-5$.

Cross, GB, Marley, J, Miles, H \& Willson, K 2001, 'Changes in nutrient intake during the menstrual cycle of overweight women with premenstrual syndrome', The British Journal Of Nutrition, Apr, Vol. 85, no. 4, pp. 475-82.

Dalton, K 1964, The Premenstrual Syndrome, Heinemann, London.

Dalton, K 1984, The premenstrual syndrome and progesterone therapy, $2^{\text {nd }}$ edn., Yearbook Medical Publishers, Chicago.

Dalton, K, Dalton, ME \& Guthrie, K 1987, 'Incidence of the premenstrual syndrome in twins', BMJ, vol. 295, pp.1027-1028.

Daugherty, JE 1998, 'Treatment strategies for premenstrual syndrome', Am Fam Physician, vol. 58, no. 1, pp. 183-92, 197-8.

de la Fuente-Fernández, R \& Stoessl, AJ 2004, 'The biochemical bases of the placebo effect', Sci Eng Ethics, vol. 10, no. 1, pp. 143-50.

de la Gandara Martin, JJ \& de Diego Herrero, E 1996, 'Premenstrual dysphoric disorder: an epidemiological study', Actas Luso-Espanolas de Neurologia. Vol. 24, pp. 111-117.

De Souza, MC, Walker, AF, Robinson, PA \& Bolland, K A 2000, 'Synergistic effect of a daily supplement for 1 month of $200 \mathrm{mg}$ magnesium plus $50 \mathrm{mg}$ vitamin B6 for the relief of anxiety-related premenstrual symptoms: a randomized, double-blind, crossover study', Journal of Women's Health \& Gender-Based Medicine, vol. 9, no. 2, pp. 131-9.

Deadman, P, Al-Khafaji, M\& Baker K 1998, A manual of acupuncture, Journal of Chinese Medicine Publications, East Sussex. 
Deng, T 1999, Practical diagnosis in traditional Chinese medicine, Churchill Livingstone, Sydney.

Deuster, PA, Adera, T \& South-Paul, J 1999, 'Biological, social, and behavioral factors associated with premenstrual syndrome', Arch Fam Med, vol. 8, pp. 122-128.

Dharmananda, S 2000, The significance of traditional pulse diagnosis in the modern practice of Chinese medicine, viewed 2 March 2006, $<$ http://www.itmonline.org/arts/pulse.htm>.

Dickerson, LM, Mazyck, PJ \& Hunter, MH 2003, 'Premenstrual syndrome', American Family Physician, vol.67, no. 8, pp.1743-51.

Diegoli, MS, da Fonseca, AM, Diegoli, CA \& Pinotti, JA 1998, ‘A double-blind trial of four medications to treat severe premenstrual syndrome', Int J Gynaecol Obstet, vol. 62, pp. 63-7.

Dimmock, PW, Wyatt, KM, Jones, PW \& O'Brien, PM 2000, 'Efficacy of selective serotonin-reuptake inhibitors in premenstrual syndrome: a systematic review', Lancet, vol. 356, pp. 1131-6.

Ding, C \& Sun, X (eds) 2003, Illustration of Tongue diagnosis of TCM, People's Medical Publishing House, Beijing.

Ding, M 1999, Diagnosis and Differential Diagnosis of Gynecological and Obstetrical Diseases (妇产科疾病诊断与鉴别诊断), $2^{\text {nd }}$ edn, People's Medical Publishing House, Beijing.

Ding, S-d, Guan, E \& Ding, S-q 2001, Treating 68 cases of premenstrual tension syndrome by Shu Gan Zhu Shen Tang' (舒肝宁神汤治疗经前期紧 张综合症 68 例), Forum On Traditional Chinese Medicine, vol. 16, no. 4, p. 15.

Dong, L 2003, 'Four methods to treat premenstrual syndrome through liver' (经前期综合征从肝论治 4 法), Zhejiang Journal of Traditional Chinese Medicine, vol. 38, no. 7, p. 284.

Dou, Y 2004, 'Treatment of 58 cases of premenstrual syndrome by modified Dan Zhi Xiao Yao San'(丹枙逍遥散加减治疗经前期综合症 58 例), Forum On Traditional Chinese Medicine, vol. 19, no. 5, p. 21. 
Douglas, S 2002, 'Premenstrual syndrome. Evidence-based treatment in family practice', Canadian Family Physician, vol. 48, pp. 1789-97.

Dye, L \& Blundell, JE 1997, 'Menstrual cycle and appetite control: implications for weight regulation', Hum Reprod, vol. 12, no. 6, pp. 1142-51.

Endicott, J 2000, 'History, evolution, and diagnosis of premenstrual dysphoric disorder', J Clin Psychiatry, vol.61 Suppl, no.12, pp.5-8.

Endicott, J \& Halbreich, U 1982, 'Retrospective reports of premenstrual depressive changes: factors affecting confirmation by daily ratings', Psychopharmacol Bull, vol. 18, no. 3, pp. 109-12.

Facchinetti, F, Borella, P, Sances, G, Fioroni, L, Nappi, RE \& Genazzani, AR 1991, 'Oral magnesium successfully relieves premenstrual mood changes', Obstet. Gynecol, vol. 78, pp. 177-181.

Fang, G 1536, The Appendix of Dan-Xi's Experiential Methods (丹溪心法附 余), China

Feng, C, Zhang, Q \& Yin, X 2005, 'Observation of treatment effect on 36 cases of premenstrual breasts distending pain with liver qi stagnation by modified Xiao Yao San' (逍遥散加减治疗肝郁气滞型经前乳房胀痛 36 例 疗效观察), Hebei Journal of Traditional Chinese Medicine, vol. 27, no. 10, p. 785 .

Flaws, B 1997, The secret of Chinese pulse diagnosis, Blue Poppy Press, Boulder, Colo.

Frank, RT 1931, 'The hormonal causes of premenstrual tension', Arch Neurol Psychiatry, vol. 26, pp. 1053-57.

Freeman, E 2003, 'Premenstrual syndrome and premenstrual dysphoric disorder: definitions and diagnosis', Psychoneuroendocrinology, vol. 28 Suppl 3, pp. 25-37.

Freeman, EW \& Halbreich, U 1998, 'Premenstrual syndromes', Psychopharmacol Bull, vol. 34, no. 3, pp. 291-5. 
Freeman, EW \& Rickels, K 1999, 'Characteristics of placebo responses in medical treatment of Premenstrual syndrome', Am J Psychiatry, vol. 156, no. 9, pp. 1403-8.

Freeman, EW, DeRubeis, RJ \& Rickels, K 1996, 'Reliability and validity of a daily diary for premenstrual syndrome', Psychiatry Res, vol. 65, pp. 97-106.

Freeman, EW, Rickels, K, Schweizer, E \& Ting, T 1995, 'Relationships between age and symptom severity among women seeking medical treatment for premenstrual symptoms', Psychol Med., vol. 25, no. 2, pp. 309-15.

Freeman, EW, Rickels, K, Sondheimer, SJ \& Polansky, M 1995, 'A double-blind trial of oral progesterone, alprazolam, and placebo in treatment of severe premenstrual syndrome', JAMA, vol. 274, pp. 51-7.

Frye, GM \& Silverman, SD 2000, 'Is it premenstrual syndrome? keys to focused diagnosis, therapies for multiple symptoms', Postgrad Med, vol.107, no.5, pp. 51-4,157-9.

Fu, H 2003, 'Treatment of 86 cases of premenstrual syndrome by modified Xiao Yao San' (逍遥散加味治疗月经前期综合征 86 例), Practical Clinical Journal of Integrated Traditional Chinese and Western Medicine, vol. 3, no. 4, p. 5.

Fu, S 1826, Fu Qing Zhu Nu Ke Fu Qing Zhu's Obstetrics and Gynecology (傅青主女科), China.

Gallant, SJ, Popiel, DA, Hoffman, DM, Chakrabortym, PK \& Hamilton, JA 1992 'Using daily ratings to confirm premenstrual syndrome/late luteal phase dysphoric disorder. Part II. What makes a "real" difference?', Psychosomatic Medicine, vol. 54, no. 2, pp. 167-81.

Gao, H 2005, 'Differential treatment of premenstrual syndrome' (中医辨证 治疗经前期紧张综合征), Journal of Henan College of Traditional Chinese Medicine, vol. 20, no. 116, pp. 45-6.

Gao, L \& Chen, W 2001, 'Treatment of 42 cases of premenstrual oral ulcer by self designed Qin Zhi Yin' (自拟芩知饮治疗经行口糜 42 例), Fujian Journal of Traditional Chinese Medicine, vol. 32, no. 6, pp. 29-30. 
Gao, S \& Chen, J 1994, 'Brief Summary of Chinese medical researches on the treatment of premenstrual syndrome with herbs' (中药治疗经前期紧张 综合征的研究概况), Journal of Traditional Chinese medicine, vol. 35, no.7, pp. 433-5.

Gao, Z 1999, Treatment of 60 cases of premenstrual breasts distension by Jie Yu Shu Gan Tang' (疏肝解郁汤治疗经前乳胀症 60 例), Zhejiang Journal of Traditional Chinese Medicine, no. 3, p. 109.

Gao, Z 2003, 'Observation of treatment effect on 46 cases of premenstrual syndrome by modified Xiao Yao San' (逍遥散加减治疗经前期综合症 46 例疗效观察), Yunan Journal of Traditional Chinese Medicine and Materia Medica, vol. 24, no. 3, p. 24.

Gise LH, Lebovits, AH, Paddison, PL \& Strain, JJ 1990, 'Issues in the identification of premenstrual syndromes', The Journal Of Nervous And Mental Disease, vol. 178, no. 4, pp. 228-34.

Goei, GS \& Abraham, GE 1983, 'Effect of a nutritional supplement, optivite, on symptoms of premenstrual tension', The Journal of reproductive medicine, vol. 28 , no. 8 , pp. $527-31$.

Goodale, IL, Domar, AD \& Benson, H 1990, 'Alleviation of premenstrual syndrome symptoms with the relaxation response', Obstet. Gynecol, vol. 75, pp. 649-655.

Gotts, G, Morse, CA \& Dennerstein, L 1995, 'Premenstrual complaints: an idiosyncratic syndrome', J Psychosom Obstet Gynaecol, Mar, vol. 16, no. 1, pp. 29-35.

Greene, R \& Dalton, K 1953, 'The premenstrual syndrome', BMJ, vol.1, pp.1007-14.

Gu, M 2004, 'Treatment of premenstrual emotional abnormalities by the combination of Hei Xiao Yao San and Xiao Chai Hu Tang' (黑逍遥散合小 柴胡汤加减治疗经行情志异常), Chinese Community Doctors, vol. 20, no. 8, p. 37.

Guo, Y 1996, 'Comparison between decocting herbs separately and together in clearing formula' (清解方单煎与共煎比较研究), Chinese Patent Medicine, vol.12. p. 42. 
Halbreich, U 1997, 'Menstrually related disorders--towards interdisciplinary international diagnostic criteria', Cephalalgia, vol. 17 Suppl 20, pp. 1-4.

Halbreich, U., 1999, 'Premenstrual syndromes: closing the 20th century chapters', Curr Opin Obstet Gynecol, vol. 11, pp. 265-270.

Halbreich, U 2003, 'The etiology, biology, and evolving pathology of premenstrual syndromes', Psychoneuroendocrinology, Aug, vol. 28 Suppl 3, pp. 55-99.

Halbreich, U 2004, 'The diagnosis of premenstrual syndromes and premenstrual dysphoric disorder--clinical procedures and research perspectives', Gynecological Endocrinology, vol. 19, no. 6, pp. 320-34.

Halbreich, U, Rojansky, N \& Palter, S, 1991, 'Elimination of ovulation and menstrual cyclicity (with danazol) improves dysphoric premenstrual syndromes', Fertil. Steril, vol. 56, pp. 1066-1069.

Halbreich, U, Rojansky, N, Palter, S, Tworek, H, Hissin, P \& Wang, K 1995, 'Estrogen augments serotonergic activity in postmenopausal women', Biol Psychiatry, vol. 37, pp. 434-441.

Halbreich, U, Sachar, EJ, Asnis, GM, Quitkin, F, Nathan, RS, Halpern, FS \& Klein, DF 1982, 'Growth hormone response to dextroamphetamine in depressed patients and normal subjects', Arch Gen Psychiatry, vol.39, no. 2, pp. 189-92.

Hamilton, JA, Parry, BL, Alagna, S, Blumenthal, S \& Herz, E 1984, 'Premenstrual mood changes; a guide to evaluation and treatment', Psychiatr Ann, vol. 14, pp. 426-435.

Han, F 1998, 'Report on 154 cases of treatment for premenstrual tension syndrome by nourish yin and replenish blood' (滋阴养血法治疗经前期紧 张综合症 154 例), Heilongjiang Journal of Traditional Chinese Medicine, vol. 4 , p. 12.

Haour, F 2005, 'Mechanisms of the placebo effect and of conditioning', Neuroimmunomodulation, vol. 12, no. 4, pp. 195-200 
Hardy, ML 2000, 'Herbs of special interest to women', J Am Pharm Assoc (Wash), vol. 40, no. 2, pp. 234-42, quiz 327-9.

Harrison, W, Endicott, J, Rabkin, JG \& Nee, J 1984, 'Treatment of dysphoric changes: clinical outcome and methodological implications', Psychol Bull, pp.118-22.

Hart, WG, Coleman, GJ \& Russell, JW 1987, 'Assessment of premenstrual symptomatology: a re-evaluation of the predictive validity of self-report', Journal of psychosomatic research, vol.31, no. 2, pp.185-90.

Haspels, AA 1981, 'A double-blind, placebo-controlled multi-centre study of the efficacy of dydrogesterone (Duphaston)', in The premenstrual syndrome Pieter A K and Wulf, HU (eds) MTP Press LTD, Lancaster, Engliand

Haywood, A, Slade, P \& King, H 2002, 'Assessing the assessment measures for menstrual cycle symptoms: a guide for researchers and clinicians', Journal Of Psychosomatic Research, vol. 52, no. 4, pp. 223-37.

He, X \& Li, M 2000, 'Treatment of 30 cases of peri-menstrual emotional abnormalities by the combination of auricular point pressing and Gan Mai Da Zao Tang' (耳压配合甘麦大本汤治疗经行情志异常 30 例), Journal of Clinical Acupuncture and Moxibustion, vol. 16, no. 5, pp. 53-4.

He, Z \& Wang, Z 1995, 'Treating premenstrual syndrome through liver' (从肝论治经前紧张综合征), Journal of Beijing University of Traditional Chinese Medicine, vol. 18, no. 6, pp. 47-48.

Health Ministry, the General Logistics Department, the Chinese People's Liberation Army (ed.) 1998, Clinical Diagnosis and Standard of Therapeutic Effect on Diseases (临床疾病诊断依据治愈好转标准), 2nd edn, People's Military Medical Press, Beijing.

Herman, SL \& Lester, D 1994, 'Physical symptoms of stress, depression and suicidal ideation in high school students', Adolescence, Fall, 1994

Herzog, AG. 1997, 'Continuous bromocriptine therapy in menstrual migraine’, Neurology, vol. 48, pp. 101-2. 
Holroyd, E, Chang, AM \& Chau, JPC 1998, 'Assessing premenstrual syndrome in Chinese society', Social Sciences in Health: International Journal of Research \& Practice, vol. 4, no. 3, pp. 143-53.

Hong, Y 2002, 'Observation of treatment effect of scalp acupuncture on premenstrual syndrome’ (头针治疗经前期综合征的疗效观察), Shanghai Journal of Acupuncture and Moxibustion, vol. 21, no. 3, p. 24.

Hsiao, MC, Hsiao, CC \& Liu, CY 2004, 'Premenstrual symptoms and premenstrual exacerbation in patients with psychiatric disorders', Psychiatry and clinical neurosciences, vol. 58. no. 2, pp. 186-90.

Hu, J, \& Mo, Y 1998, 'Clinical significance of changes in the content of sEDF between Heart qi xu pattern in palpitation and blood stagnation'(心悸 病心气虚证与心血㾉组证患者 sEDF 含量变化的临床意义), Chinese Journal of the Practical Chinese with Modern Medicine, vol. 11, no. 10, p.888.

Hua, Z 2000, 'Experience in the treatment of 36 cases of peri-menstrual emotional abnormalities by Gui Pi Tang' (归脾汤治疗经行情志异常 36 例 体会), Gansu Journal of Traditional Chinese Medicine, no. 2, pp. 41-2.

Huang, H 2000b, 'Experience in the treatment of premenstrual feverish sensation by modified Xiao Chai Hu Tang' (小柴胡汤加减治疗经期发热体 会), Chinese Journal of Information on TCM, vol. 7, no. 11, p. 69.

Huang, L 1999, 'Clinical observation of treatment of period pain and premenstrual syndrome with Kunyuening in 68 cases' (坤月宁治痛经及经 前期综合征 68 例临床观察), Jiangxi Journal of Traditional Chinese Medicine, vol. 30, no. 1, p. 30.

Huang, M 2000a, 'Treatment of 30 cases of premenstrual headache by pattern identification' (辨证治疗经期头痛 30 例), Sichuan Journal of Traditional Chinese Medicine, vol. 18, no. 7, pp. 39-40.

Huang, T (ed.) 1994, A Handbook of the Composition and Pharmcology of Common Chinese Drugs (常用中药成分与药理手册), Chinese Medical \& Pharmaceutical Scientic and technology Publishing House, Beijing. 
Huang, X \& Zhou, X 1998, 'The relation between the heart qi patterns identification in heart diseases and the content of zinc, copper, iron and calcium in blood serum'(心病心气辨证与血清锌铜铁钙含量的关系), China Journal of Basic Medicine In Traditional Chinese Medicine, vol.4, no.3, p.41.

Hubei College of Traditional Chinese Medicine (ed.) 1980, Gynecology of TCM (中医妇科学), Shanghai Science and Technology Press, China.

Hunter, MS, Ussher, JM, Browne, SJ, Cariss, M, Jelley, R \& Katz, M 2002, 'A randomized comparison of psychological (cognitive behavior therapy), medical (fluoxetine) and combined treatment for women with premenstrual dysphoric disorder', J Psychosom Obstet Gynaecol, vol. 23, no. 3, pp. 193-9.

Huo, Q, 1995, ' The application of liver regulating method for premenstrual tension syndrome' (调肝法在经前期紧张综合征中的应用), Journal of Henan University of Chinese Medicine, vol.10, no. 2, pp. 38-9.

Hylan, TR, Sundell, K \& Judge, R 1999, 'The impact of premenstrual symptomatology on functioning and treatment-seeking behavior: experience from the United States, United Kingdom, and France', Journal Of Women's Health \& Gender-Based Medicine, Oct, vol. 8, no. 8, pp. 1043-52.

Institute-of-Medical-Information 2000, Chinese Bio-Medicine Disc (CBM Disc), Chinese Academy of Medical Science, China.

Ismail KMK \& O'Brien, S 2005, 'Premenstrual syndrome', Current Obstetrics \& Gynaecology, vol. 15, Issue 1, February, pp. 25-30.

Israel, SL 1938, 'Premenstrual tension', JAMA, vol. 110, p. 1721.

Janiger, O, Riffenburgh, R \& Kersh, R 1972, 'Cross cultural study of premenstrual symptoms', Psychosomatics Jul-Aug; Vol. 13, no.4, pp. 226-35.

Jiang, H, Lin, Y \& Wang, A 1995, 'Treatment of 100 cases of period pain and premenstrual tension syndrome by Hu Gong Tiao Jing Bao Yuan bag' (护宫调经保元袋”治疗痛经、经前期紧张综合征 100 例), Journal of Beijing University of Traditional Chinese Medicine, vol. 18, no. 6, pp. 49-50. 
Jiang, W, Li, Y \& Sun, J 2002, 'Clinical study on treatment of premenstrual syndrome with auricular point picking' (耳穴贴压治疗经前期紧张综合征临 床研究), Chinese Acupuncture and Moxibustion, vol. 22, no. 3, pp. 165-7.

Jiang, Y 2003, 'Treatment of 84 cases of premenstrual acne by modified Long Dan Xie Gan Tang' (龙胆泻肝汤加减治疗经前期痤疮 84 例), New Journal of Traditional Chinese, vol. 35, no. 9, pp. 55-6.

Johnson, TM 1987, 'Premenstrual syndrome as a western culture-specific disorder', Cult Med Psychiatry, vol.11, no.3, pp.337-56.

Johnson, SR 1998, 'Pre-menstrual syndrome therapy', Clin Obstet Gynecol, vol. 41 , no. 2 , pp. $405-21$.

Kendall, PC \& Grove, WM 1988 'Normative comparisons in therapy outcome', Behavioral Assessment, vol. 10, pp. 147-158.

Kessel, B 2000, 'Premenstrual syndrome: advances in diagnosis and treatment', Obstet Gynecol Clin North Am, vol. 27, no. 3, pp. 625-39.

Kim, H 2004 'Perimenstrual symptoms of Korean women living in the USA: applicability of the WDHD(Women's Daily Health Diary) on prospective report', Taehan Kanho Hakhoe Chi, vol. 34, no. 8, pp. 1395-401.

Kirkby, RJ 1994, Changes in premenstrual symptoms and irrational thinking following cognitive behavioral coping skills training', J. Consult. Clin. Psychol, vol. 62, pp. 1026-32.

Kirkby, RJ \& Lindner, H 1998, 'Exercise is linked to reductions in anxiety but not premenstrual syndrome in women with prospectively-assessed symptoms', Psychology, Health \& Medicine, vol 3, no. 2, pp. 211-222.

Kleijnen, J, Ter Riet, G \& Knipschild, P 1990, 'Vitamin B6 in the treatment of the premenstrual syndrome--a review', Br J Obstet Gynaecol, vol. 97, pp. 847-52.

Knight, RG 1984, 'Some general population norms for the short form Beck Depression Inventory', Journal of clinical psychology, vol. 40, no. 3, pp. 751-3. 
Korzekwa, MI, Lamont, JA \& Steiner, M 1996, 'Late luteal phase dysphoric disorder and the thyroid axis revisited', The Journal Of Clinical Endocrinology And Metabolism, Jun, vol. 81, no. 6, pp. 2280-4.

Kou, S \& Wang, W 2001, 'Treatment of 52 cases of premenstrual syndrome by the integration of Chinese and western medicine' (中西医结合治疗经前 期综合征 52 例), Journal of Practical Traditional Chinese Internal Medicine, vol. 15 , no. 4 , p. 36 .

Kritz-Silverstein, D, Wingard, DL \& Garland, FC 1999, 'The Association of Behavior and Lifestyle Factors with Menstrual Symptoms', Journal of Women's Health \& Gender-Based Medicine, vol. 8, Issue 9, pp. 1185-94.

Kuang, A, Gu, D and Gu, T 1984, 'An experimental study on yin and yang of TCM'(中医阴阳的实验性研究), Chinese journal of Integrated

Traditional and Western Medicine, vol. 4, no.12, p.7421.

Kuo, C, See, L, Hsu, L \& Lee, S 1997, 'A survey investigation of premenstrual discomfort among Chinese nurses’, Nursing Research (China), vol. 5, no. 2, pp. 149-59.

Lauritzen, C, Reuter, HD, Repges, R, Bohnert KJ \& Schmidt, U 1997, 'Treatment of premenstrual tension syndrome with Vitex agnus castus. Countrolled, double-blind study versus pyridoxin', Phytomedicine, vol. 4, pp. 183-189.

Le, J 1997, The Gynaecology and Obstetrics (妇产科学), $4^{\text {th }}$ edn, People's Medical Publishing House, Beijing.

Le, J 2000, The Gynaecology and Obstetrics (妇产科学), $5^{\text {th }}$ edn, People's Medical Publishing House, Beijing.

Legros, S, Foidart, JM, Gaspard, U \& Legros, JJ 1999, 'Premenstrual tension syndrome or premenstrual dysphoria', Revue médicale de Liège (French), vol. 54, no. 4, pp. 268-73.

Lepage, P \& Steiner, M. 1991, 'Gender and serotonergic dysregulation: implications for late luteal phase dysphoric disorder' in GB Cassano \& HS Akiskal (eds.) Serotonin-Related Psychiatric Syndromes:Clinical and Therapeutic Links, pp. 131-43, R. Soc. Med. Serv., London. 
Li, C, Zhang, X \& Zhu, Y 2002, “ Treatment of 400 cases of premenstrual syndrome by Jie Yu Xiao Zhang Jian, with additional 200 cases of western medicine control group' (解郁消胀煎治疗经前期综合征 400 例-附西药对 照组 200 例), Liaoning Journal of Traditional Chinese Medicine, vol. 29, no. 4, pp. 209-10.

Li, S 2000, Treating 24 cases of peri-menstrual diarrhoea by Shen Ling Bai Zhu San'(参苓白术散治疗经行泄泻 24 例), Journal of Traditional Chinese Medicine and Chinese Materia Medica of Jilin, no. 1, p. 40.

Li, W 2000, 'Treatment of 86 cases of premenstrual breasts distension by Xiao Zhang Yin' (消胀饮治疗经前乳胀 86 例), Hebei Journal of Traditional Chinese Medicine, vol. 22, no. 5, p. 366.

Li, X 1999, Treating 58 cases of premenstrual tension syndrome by Shu Gan Jian Pi Tang' (疏肝健脾汤治疗经前期紧张综合征 58 例), Journal of Anhui Traditional Chinese Medical College, vol. 18, no. 4, p. 41.

Li, X-h, Li X-y \& Lin, S 2002, 'Treatment of 40 cases of premenstrual headache through applying liver coursingand nourishingmethod' (疏肝养肝 法治疗经行头痛 40 例), Fujian Journal of Traditional Chinese Medicine, vol. 33, no. 1, p. 29.

Li, Y 1624, Elementary Course for Medicine (医学入门), China.

Li, Z, Zhang, Y \& Tang, Q 2003, 'Analysis of the treatment of premenstrual syndrome by Jing Qian Shu Ye’ (经前舒液治疗经前期综合征50例分析), Study Journal of Traditional Chinese Medicine, vol. 21, no. 10, pp. 1749-50.

Liang, C, Geng, S \& Li, C 1996, 'Treatment of 45 cases of premenstrual breasts distension by Shu Gan Zhu Yu Tang' (疏肝逐病汤治疗经前乳房胀 痛 45 例), Hebei Journal of Traditional Chinese Medicine, vol. 18, no. 2, p. 14.

Liang, X 1996, 'Clinical observation of TCM treatment effect on premenstrual tension syndrome 60 cases' (中医治疗经前期紧张综合征 60 例临床疗效观察), Yunan Journal of Traditional Chinese Medicine and Materia Medica, vol. 17, no. 5, pp. 9-10. 
Lin, Y 2005, 'Treatment of 18 cases of premenstrual feverish sensation by modified Xiao Chai Hu Tang' (小柴胡汤加减治疗经行发热 18 例), Journal of Practical Traditional Chinese Medicine, vol. 21, no. 6, pp. 356-7.

Lin, Z 2000, 'Treating 100 cases of peri-menstrual headache by Dang Gui Si Ni Tang'(当归四逆汤治疗经期头痛 100 例), Zhejiang Journal of Traditional Chinese Medicine, no. 10, p. 423.

Liu, C 2000, Treating 46 cases of peri-menstrual swelling by Ling Gui Zhu Gan Tang' (苓桂术甘汤治疗经行浮肿 46 例), Journal of Traditional Chinese Medicine and Chinese Materia Medica of Jilin, no. 3, p. 25.

Liu, S (ed.) 1921, Ye Tian-Shi's Gynecological and Obstetrical Records (叶 天士女科医案), China.

Liu, Y \& Wang, X 2002, 'Treatment of 30 cases of peri-menstrual emotional abnormalities by the combination of Chinese medicine and psychological therapy' (中医药内服配合心理疏导治疗经行情志异常心血不足型30例), Yunan Journal of Traditional Chinese Medicine and Materia Medica, vol. 23, no. 2, p. 18.

Liu, Y, Zhang, R \& Dong, L 1995, Advanced textbook on TCM and pharmacology: Book two Basic theory of TCM traditional Chinese medicine, New World Press, Beijing, China.

LoBiondo-Wood, G \& Haber, J 1990, Nursing research: method, critical appraisal, and utilization, $2^{\text {nd }}$ edn, The C. V. Mosby Co, St. Louis.

London, RS, Murphy, L, Kitlowski, KE \& Reynolds, MA 1987, 'Efficacy of alpha-tocopherol in the treatment of the premenstrual syndrome', The Journal Of Reproductive Medicine, vol. 32, no. 6, pp. 400-4.

London, RS, Bradley, L \& Chiamori, NY 1991, 'Effect of a nutritional supplement on premenstrual symptomatology in women with premenstrual syndrome: a double blind longitudinal study', J Am Coll Nutr, vol. 10, pp. 494-9.

Long, PW 1995, Internet Mental Health, viewed 1 March 2005, $<$ http://www.mentalhealth.com>. 
López-Rosales, C, Aguilar-Guerrero, JA \& García-Moreno, L 1996, 'Treatment of premenstrual tension syndrome with lisuride maleate', Ginecología y obstetricia de México (México), vol. 64, pp. 556-60.

Lu Q 2001, 'Treatment of 52 cases of premenstrual tension syndrome by Jing Qian Shu Tang' (经前舒汤治疗经前期紧张症 52 例), Yunan Journal of Traditional Chinese Medicine and Materia Medica, vol. 22, no. 4, p. 34.

Luo, B 1989, 'Experience in the treatment of 36 cases of premenstrual feverish sensation' (经前发热 36 例证治体会), Shanxi Journal of Traditional Chinese Medicine, vol. 5, no. 1, pp. 21-2.

Luo, Y (ed.) 1986, The Gynecology of TCM (中医妇科学), 5th edn, Shanghai Science and Technology Press, China.

Luo, Y (ed.) 1994, Practical Gynecology of TCM (实用中医妇科学), Shanghai Science and Technology Press, China.

Lustyk, MK, Widman, L, Paschane, A \& Ecker, E 2004, 'Stress, quality of life and physical activity in women with varying degrees of premenstrual symptomatology', Women \& health, vol. 39, no. 3, pp. 35-44.

Ma, B (ed.) 1997, The Gynecology of TCM (中医妇科学), 6th edn, Shanghai Science and Technology Press, China.

Ma, Z 2004, "Treatment of 78 cases of premenstrual syndrome by Chinese herbal medicine' (中药治疗经前期综合症 78 例小结), Journal of Guiyang College of Traditional Chinese Medicine, vol. 26, no. 2, p. 35.

Maciocia, G 1989, The foundations of Chinese medicine: a comprehensive text for acupuncturists and herbalists, Churchill Livingstone, Melbourne.

Markum, RS 1976, 'Assessment of the reliability of the effect of neutral instructions on the symptom ratings on the moos menstrual distress questionnaire', Pshchosomatic Med, vol.38, no.3, pp. 163-72.

Marván, ML, Díaz-Erosa, M \& Montesinos, A 1998, 'Premenstrual symptoms in Mexican women with different educational levels', The Journal of psychology, vol. 132. no. 5, pp. 517-26. 
Masho, SW, Adera, T \& South-Paul, J 2005, 'Obesity as a risk factor for premenstrual syndrome', Journal of Psychosomatic Obstetrics \& Gynecology, vol. 26, Issue 1, p.33-9.

Matsumoto, A, Arai, Y \& Osanai, M 1985, 'Estrogen stimulates neuronal plasticity in the deafferented hypothalamic arcuate nucleus in aged female rats', Neurosci Res, vol. 2, pp. 412-418.

McEwen, BS, Alves, SE \& Bulloch, K 1997, 'Ovarian steroids and the brain: Implications for cognition and aging', Neurology, vol. 48 Suppl 7, S8-S15.

McLellan, AT \& Hunkeler, E 1998, 'Patient satisfaction and outcomes in alcohol and drug abuse treatment', Psychiatric Services, vol. 49, pp.573-75.

MedicineNet.com 2007, MedTerms Medical Dictionary [online], MedicineNet, Inc. California, viewed at 28 April 2007, <http://www.medterms.com/script/main/art.asp?articlekey=33520>.

Mersy, DJ 1991, 'Health benefits of aerobic exercise', Postgraduate Medicine, vol. 90, no. 1, pp. 103-7, 110-2.

Miller, MN, McGowen, KR, Miller, BE, Coyle, BR \& Hamdy, R 1999, 'Lessons learned about research on premenstrual syndrome', Journal Of Women's Health \& Gender-Based Medicine, vol. 8, no. 7, pp. 989-93.

Ministry of Health, the Professional Standards of Chinese Medicine of the State Administration of TCM 1997, The Guidelines for Clinical Research in New TCM Medicines(中药新药临床研究指导原则), 3rd edn, The Peoples' Republic of China.

Mira, M, Stewart, PM \& Abraham, SF 1988, 'Vitamin and trace element status in premenstrual syndrome', Am J Obstet Gynecol, vol. 54, no. 4, pp. 643-7.

Mishra, GD, Dobson, AJ \& Schofield, MJ 2000, 'Cigarette smoking, menstrual symptoms and miscarriage among young women', Aust N Z J Public Health, vol.24, p. 413. 
Mo, L 1999, 'Clinical observation of treatment of premenstrual oral ulcer by self designed formula Er Di Qin Lian Yin in 40 cases' (自拟二地芩连饮治疗 经行口糜40例临床观察), Forum On Traditional Chinese Medicine, vol. 14, no. 3 , p. 25 .

Moos, RH 1968, 'The development of a menstrual distress questionnaire', Psychosomatic Medicine, vol. 30, no. 6, pp. 853-67.

Moos, RH 1969, Menstrual distress questionnaire preliminary manual, Social Ecology Laboratory, Department of Psychiatry and behavioural Sciences, Stanford University and Veterans Administration medical Center, Palo Alto, CA.

Moos, RH 1985, Perimenstrual symptoms: a manual and overview of research with the menstrual distress questionnaire, Social Ecology Laboratory, Department of Psychiatry and behavioural Sciences, Stanford University and Veterans Administration medical Center, Palo Alto, CA.

Morse, CA \& Dennerstein, L 1988, 'The factor structure of symptom reports in premenstrual syndrome', J Psychosom Res, vol.32, no.1, pp.93-8.

Morse, CA, Dennerstein, L, Farrell, E \& Varnavides, K 1991, 'A comparison of hormone therapy, coping skills training, and relaxation for the relief of premenstrual syndrome', J. Behav. Med, vol. 14, pp. 469-89.

Mortola, JF, Girton, L, Beck, L \& Yen, SSC 1990, 'Diagnosis of premenstrual syndrome by a simple, prospective, and reliable instrument: The calendar of premenstrual experiences', Obstet Gynecol, vol.76, pp. 302-7.

Mortola, JF, Girton, L \& Fischer, U 1991, 'Successful treatment of severe premenstrual syndrome by combined use of gonadotropin-releasing hormone agonist and estrogen/progestin', J. Clin. Endocrinol. Metab, vol. 72, pp. 252A-252F.

$\mathrm{Mu}, \mathrm{C} 2004$, 'Treatment of premenstrual migraine by Chinese medicine' (中 药治疗经前偏头痛), Shanxi Journal of Traditional Chinese Medicine, vol. 20 , no. 3, p. 7.

National Health \& Medical Research Council (NHMRC) 1992, National Health and Medical Research Council Act 1992, NHMRC, Commonwealth of Australia. 
National Health \& Medical Research Council (NHMRC) 1999, National

Statement on Ethical Conduct in Research Involving Humans, NHMRC, Commonwealth of Australia.

National Health \& Medical Research Council (NHMRC) 2001, Australia Alcohol Guidelines: Health Risks and Benefits, NHMRC, Commonwealth of Australia.

Nikolai, TF, Mulligan, GM, Gribble, RK, Harkins,PG, Meier, PR \& Roberts, RC 1990, 'Thyroid function and treatment in premenstrual syndrome', The Journal Of Clinical Endocrinology And Metabolism, Apr, vol. 70, no. 4, pp. 1108-13.

Niu, J (ed.) 1996, Modern Gynecology in Chinese and Western Medicine (现 代中西医妇科学), China Science and Technology Press, Beijing.

O’Brien, PMS 1987, Premenstrual syndrome, Blackwell Scientific Publications, London.

O'Brien, PMS \& Abukhalil, IEH 1999, 'Randomized controlled trial of the management of premenstrual syndrome and premenstrual mastalgia using luteal phase-only danazol', Am. J. Obstet. Gynecol, vol. 180, pp. 18-23.

O'Brien, PM, Craven, D, Selby, C \& Symonds, EM 1979, 'Treatment of premenstrual syndrome by spironolactone', Br J Obstet Gynaecol, vol. 86, no. 2 , pp. 142-7.

Oguchi, T 2003, 'Premenstrual tension syndrome', Ryoikibetsu Shokogun Shirizu (Japanese ), vol. 38, pp. 571-4.

Ossege, M, Sycha, T, Aigner, M, Schmetterer, L, Eichler, HG, Müller, M, König, F \& Bauer, P 2005, 'Effect of information on reported adverse events in a placebo-controlled trial', Drug Saf, vol. 28, no. 1, pp. 81-7.

Parker, PD 1994, 'Premenstrual syndrome', American Family Physician, vol.50, no.6, pp.1309-20.

Parry, BL 1994, 'Biological correlates of premenstrual complaints', in JH Gold and SK Severino (eds.) Premenstrual Dysphorias, American Psychiatric Press, Washington, D.C. 
Pearlstein, TB, Bachmann, GA, Zacur, HA \& Yonkers, KA 2005a, 'Treatment of premenstrual dysphoric disorder with a new drospirenone-containing oral contraceptive formulation', Contraception, vol. 72 , no. 6 , pp. $414-21$.

Pearlstein, T, Yonkers, KA, Fayyad, R \& Gillespie, JA 2005, 'Pretreatment pattern of symptom expression in premenstrual dysphoric disorder', Journal of affective disorders, vol. 85, no. 3, pp. 275-82.

Pearlstein, TB, Halbreich, U, Batzar, ED, Brown, CS, Endicott, J, Frank, E, Freeman, EW, Harrison, WM, Haskett, RF, Stout, AL \& Yonkers, KA 2000, 'Psychosocial functioning in women with premenstrual dysphoric disorder before and after treatment with sertraline or placebo', The Journal of Clinical Psychiatry, vol. 61, no. 2, pp. 101-9.

Peng, L 1999, 'Treating 38 cases of peri-menstrual rashesby Bu Shen Rou Gan Xiao Zhen Tang' (补肾柔肝消疹汤治疗月经疹 38 例), Hunan Guiding Journal of Traditional Chinese Medicine and Pharmacology, vol. 5, no. 2, pp. 25-6.

Perry, BL, Miles, D, Burruss, K \& Svikis, DS 2004, 'Premenstrual symptomatology and alcohol consumption in college women', J Stud Alcohol, Jul, vol. 65, no. 4, pp. 464-8.

Prior, JC, Vigna, Y, Sciarretta, D, Alojado, N \& Schulzer, M 1987, 'Conditioning exercise decreases premenstrual symptoms: a prospective controlled 6-month trial', Fertil Steril, vol. 47, pp. 402-8.

Qiao, M, Zhang, H, Jiang, K, Fu, Y, Wang, J, Dai, S, Yuan, L \& Cui, T 2002, 'Efficacy and safety of Jingqianping granule in treatment of 403 cases of the liver invasion of premenstrual syndrome with multicentre, random, double blind and double simulation and control method' (经前平颗粒多中心、随机 双盲双模拟对照治疗经前期综合征肝气逆证 403 例), Chinese New Drugs Journal, vol. 11, no. 5, pp. 389-92.

Qiao, M, Zhang, Z, Xu, X, Han, X ,Ci, Y \& Ye, Q 1997, 'Epidemiological survey on syndromes distribution in PMS' (经前期综合征证候分布规律的 流行病学调查研究), China Journal of Basic Medicine In Traditional Chinese Medicine, vol. 3,no. 3, pp. 31-3, 64. 
Qiu, M 2003, 'Treatment of 78 cases of premenstrual distension headache by eyetension syndrome by modified Long Dan Xie Gan Tang' (加味龙胆泻肝 汤治疗经前期头目胀痛 78 例), New Journal of Traditional Chinese, vol. 35 , no. 4, pp.61-2.

Ramcharan, S, Love, EJ, Fick, GH \& Goldfien A 1992, 'The epidemiology of premenstrual symptoms in a population-based sample of 2650 urban women: attributable risk and risk factors', J Clin Epidemiol, vol. 45, pp.377-392.

Ran, J 1997, 'Treatment of 37 cases of premenstrual tension syndrome by auricular point pressing' (耳穴贴压治疗经前期紧张综合征 37 例), Chinese Acupuncture and Moxibustion, vol. 17, no. 7, p. 434.

Rapkin, A. 1992, 'The role of serotonin in premenstrual syndrome', Clin. Obstet. Gynecol, vol. 35. pp. 629-36.

Rapkin, A 2003, 'A review of treatment of premenstrual syndrome and premenstrual dysphoric disorder', Psychoneuroendocrinology, vol. 28 Suppl 3, pp. 39-53.

Reid, RL, Greenaway-Coates, A \& Hahn, PM 1986, 'Oral glucose tolerance during the menstrual cycle in normal women and women with alleged premenstrual "hypoglycemic" attacks: effects of naloxone', J Clin Endocrinol Metab, Jun, vol. 62, no. 6, pp. 1167-72.

Remor, EA \& Carrobles, JA 2001, 'The Perceived Stress Scale. Psychometric Study with Spanish HIV+ Sample', in 22nd International Conference STAR, 12-14 July 2001. Palma de Mallorca, Spain.

Reynolds, WM \& Gould, JW 1981, 'A psychometric investigation of the standard and short form Beck Depression Inventory', Journal of Consulting and Clinical Psychology, vol. 49, no. 2, pp. 306-7.

Richardson, JTE 1990, 'Questionnaire studies of paramenstrual symptoms', Psychol Women Q, vol.14, pp.15-42.

Richardson, JTE 1995, 'The premenstrual syndrome: A brief history', Social Science \& Medicine, Vol.41, Issue 6, September, pp. 761-767. 
Richter, MA, Haltvick, R \& Shapiro, S 1984, 'Progesterone treatment of premenstrual syndrome', Current Therapeutic Research, vol. 36, no. 5, Sect 2, pp. 840-50.

Robinson, RL \& Swindle, RW 2000, 'Premenstrual symptom severity: impact on social functioning and treatment-seeking behaviors', Journal of Women's Health \& Gender-Based Medicine, vol. 9, no. 7, pp. 757-68.

Rosnow, RL \& Rosenthal, R 1996, 'Computing contrasts, effect sizes, and counternulls on other people's published data: General procedures for research consumers', Psychological Methods, vol. 1, pp.331-340.

Rossignol, AM \& Bonnlander, H 1990, 'Caffeine-containing beverages, total fluid consumption, and premenstrual syndrome', Am J Public Health, Sep, vol. 80, no. 9, pp. 1106-10.

Rubinow, DR \& Schmidt, PJ 1995, 'The treatment of premenstrual syndrome - forward into the past', N. Engl. J. Med, vol. 332, pp. 1574-75.

Sayegh, R, Schiff, I, Wurtman, J, Spiers, P, McDermott, J \& Wurtman, R 1995, 'The effect of a carbohydrate-rich beverage on mood, appetite, and cognitive function in women with premenstrual syndrome', Obstet. Gynecol, vol. 86 , pp. 520-528.

Schellenberg, R 2001, 'Treatment for the premenstrual syndrome with agnus castus fruit extract: prospective, randomised, placebo controlled study', $\mathrm{Br}$. Med. J, vol. 322, pp. 134-137.

Schmidt, PJ, Grover, GN, Roy-Byrne, PP \& Rubinow, DR 1993, 'Thyroid function in women with premenstrual syndrome', The Journal Of Clinical Endocrinology And Metabolism, Mar, vol. 76, no. 3, pp. 671-4.

Serverino, SK \& Moline, ML 1989, Premenstrual syndrome: a clinicians guide, The Guilford Press, New York.

Shao, M \& Guo, Y 2005, 'Treatment of 98 cases of premenstrual syndrome by the integration of Chinese and western medicine' (中西医结合治疗经前 期综合征 98 例)

Sichuan Journal of Traditional Chinese Medicine, vol. 23, no. 12, pp. 71-2. 
Shin, SS 1999, 'Development of integrated traditional Chinese and western medicine and change of medical policy in China', Uisahak, vol. 8, no. 2, pp. 207-32.

SiTu, Y. \& Yang, J. 2000, Chines medical diagnosis and treatment method of gynecology (妇科专病中医临床诊治), People's Medical Publishing House, Beijing.

Smith, SL 1975, 'Mood and the menstrual cycle'. In EJ Sacher (ed.) Topics in Psychoendocrinology, Grune and Stratton, New York,

Song, M 1996, 'Peri-menstrual vomiting' (经行呕吐), Shandong Journal of Traditional Chinese Medicine, vol. 15, no. 12, p. 537.

Spielberger, CD 1979, Preliminary Manual for the state-trait personality inventory (STPI), University of South Florida, Tampa

Spilker, B 1996, Guide to clinical trials, Lippincott-Raven Publishers, Philadelphia.

SPSS (Statistical Package for the Social Sciences), version 11.0, 2001, CD-ROM, SPSS Inc, Chicago, Illinois.

State Administration of Traditional Chinese Medicine 1994, Professional Standards of Chinese Medicine for The People's Republic China (中华人民 共和国中医药行业标准), Nanjing University Press, China.

Steege, JF \& Blumenthal, JA 1993, 'The effects of aerobic exercise on premenstrual symptoms in middle-aged women: a preliminary study', $J$ Psychosom Res, vol. 37, pp. 127-33.

Steiner, M 1997, 'Premenstrual syndromes', Annual Review Of Medicine, vol. 48, pp. 447-55.

Steiner, M, Brown, E, Trzepacz, P, Dillon, J, Berger, C, Carter, D, Reid, R \& Stewart, D 2003, 'Fluoxetine improves functional work capacity in women with premenstrual dysphoric disorder', Arch Womens Ment Health, vol. 6, no. 1, pp. 71-7.

Steiner, M 2000, 'Recognition of PMDD and its treatment', The Lancet, vol.356, pp. 1126-7. 
Steiner, M, Streiner, DL \& Pham, B 2005 'Validation of a revised visual analog scale for premenstrual mood symptoms: results from prospective and retrospective trials', Canadian journal of psychiatry, vol. 50, no. 6, pp. 327-32.

Stewart, A 1987, 'Clinical and biochemical effects of nutritional supplementation on the premenstrual syndrome', J Reprod Med, vol. 32, pp. 435-41.

Stewart-Williams, S \& Podd, J 2004, 'The placebo effect: dissolving the expectancy versus conditioning debate', Psychol Bull, vol. 130, no. 2, pp. 324-40.

Sternfeld, B, Swindle, R, Chawla, A, Long, S \& Kennedy, S 2002, 'Severity of premenstrual symptoms in a health maintenance organization population', Obstet Gynecol, vol. 99, pp. 1014-24.

Stolberg, M 2000, 'The monthly malady: a history of premenstrual suffering', Medical History, vol. 44, no. 3, pp. 301-22.

Strine, TW, Chapman, DP \& Ahluwalia, IB 2005, 'Menstrual-related problems and psychological distress among women in the united states', $J$ Womens Health (Larchmt), vol. 14, no. 4, pp. 316-23.

Sun, H 2003, 'Treatment of 48 cases of premenstrual migraine by Chinese medicine' (中药治疗经前偏头痛 48 例), New Journal of Traditional Chinese, vol. 35, no. 2, pp. 52-3.

Sun, Y \& Guo, S 2004, 'Comparative analysis of the effectiveness of acupuncture andmedication for the treatment of premenstrual syndrome' (针 刺与药物治疗经前期综合征疗效对比), Chinese Acupuncture and Moxibustion, vol. 24, no. 1, pp. 29-30.

Sun Ten 2001 Newsletter Dec 2001 Sun Ten Chinaherb Co Aus

Sundstrom, I, Nyberg, S, Bixo, M, Hammarback, S \& Backstrom, T 1999, 'Treatment of premenstrual syndrome with gonadotropin-releasing hormone agonist in a low dose regimen', Acta Obstet Gynecol Scand, vol. 78, pp. 891-9. 
Sveindottir, H \& Backstrom, T 2000, 'Prevalence of menstrual cycle symptom cyclicity and premenstrual dysphoric disorder in a random sample of women using and not using oral contraceptives', Acta Obstetricia et Gynecologica Scandinavica, vol. 79, Issue 5, pp. 405-13.

Sveinsdottir, H \& Marteinsdottir, G 1991, 'Retrospective assessment of premenstrual changes in Icelandic women', Health Care Women Int, vol. 12, no. 3 , pp. 303-15.

Tan, J \& Li, Y 1999, 'Clinical observation of treatment of premenstrual oral ulcer in 43 cases’ (疏肝活血冲剂治疗经前口疮43例临床观察), Chinese Journal of Traditional Medical Science and Technology, vol. 6, no. 2, pp. 122-3.

Tang H. 2005, 'Observation of treatment effect on 60 cases of premenstrual tension syndrome by dealing with the liver failing to disperse and purge' (从 肝失疏泄论治经前期紧张症 60 例疗效观察), Yunan Journal of Traditional Chinese Medicine and Materia Medica, vol. 26, no. 3, pp.18-9.

Tatarchuk, T, Solsky, J, Shevchuk, T \& Bodryagova, O 1999, 'Premenstrual syndrome in different age groups', 9th International Menopause Society World Congress on the Menopaus, Yokohama, Japan, October 1999. PG. pp. 65-68.

Taylor, DL, Mathew, RH, Ho, BT \& Weinman, ML. 1984, 'Serotonin levels and platelet uptake during premenstrual tension', Neuropsychobiology, vol. 12, pp. 16-18.

Taylor, DL 1994, ' Evaluating therapeutic change in symptom severity at the level of the individual women experiencing severe PMS', Image J Nurs Sch, vol. 26, no. 1, pp. 25-33.

Tesch, BJ 2003, 'Herbs commonly used by women: an evidence-based review', American Journal Of Obstetrics And Gynecology, vol. 188, Suppl 5, pp. S44-55.

Thys-Jacobs, S, Alvir, JMJ \& Fratacangelo, P 1995, 'Comparative analysis of three PMS assessment instruments - the identification of premenstrual syndrome with core symptoms', Psychopharm Bull, vol. 31, pp. 389-96. 
Thys-Jacobs, S, Starkey, P, Bernstein, D \& Tian, J 1998, 'Calcium carbonate and the premenstrual syndrome: effects on premenstrual and menstrual symptoms', Am J Obstet Gynecol, vol. 179, pp. 444-52.

Tian, D \& Zou J 2000, Traditional Chinese Internal Mrdicine (中医内科学), Academy Press, Beijing.

Tian, P, Jiang, J \& Bi, Q 2003, Report on 76 cases of treatment of premenstrual breasts distension with Shu Gan San' (疏肝散治疗经前乳胀 76 例报告), Shandong Medical Journal, vol. 43, no. 28, p.65.

Trochim, W 2000, The Research Methods Knowledge Base, 2nd edn, Atomic Dog Publishing, Cincinnati, $\mathrm{OH}$.

Turner, PD \& Pol, LG 1995, 'Beyond patient satisfaction: Broaden the scope of quality measurement by integrating the marketing view with other perspectives', Journal Of Health Care Marketing, vol. 15, no. 3, pp. 45-53.

Ugarriza, DN, Klingner, S \& O'Brien, S 1998, 'Premenstrual syndrome: diagnosis and intervention', The Nursing Practitioner, Vol. 23, no. 9, pp. $40,45,49-50$.

van der Ploeg HM \& Lodder EB 1993, 'Longitudinal measurement in the diagnostics of the premenstrual syndrome', Journal of Psychosomatic Research, vol. 37, no. 1, pp. 33-8.

Vickers, A, Goyal, N, Harland, R \& Rees, R 1998, 'Do Certain Countries Produce Only Positive Results? A Systematic Review of Controlled Trials', Controlled Clinical Trials, vol. 19, Issue 2, pp. 159-66.

Walker, AF, De Souza, MC, Vickers, MF, Abeyasekera, S, Collins, ML \& Trinca, LA 1998, 'Magnesium supplementation alleviates premenstrual symptoms of fluid retention', Journal Of Women's Health, vol. 7, no. 9, pp. 1157-65.

Walsh, MJ \& Polus, BI 1999, 'A randomized, placebo-controlled clinical trial on the efficacy of chiropractic therapy on premenstrual syndrome', Journal Of Manipulative And Physiological Therapeutics, vol. 22, no. 9, pp. 582-5. 
Wang, B 2002, 'Treating 39 cases of premenstrual syndrome by the method of coursing liver, resolving stagnation and strengthening spleen' (疏肝解郁健 脾法治疗经前期综合征39例), Fujian Journal of Traditional Chinese Medicine, vol. 33, no. 4, pp.28-9.

Wang, J 2000, 'Treatment of premenstrual tension syndrome by liver coursing and menstruation regulating method' (疏肝调经法治疗经前期紧张综合征), Jiangxi Journal of Traditional Chinese Medicine, vol. 31, no. 6, p. 62.

Wang, J \& Cui, L 1997, 'Treatment of 62 cases of premenstrual tension syndrome by modified Xiao Yao San' (逍遥散加减治疗经前期紧张综合征 62 例), Shaanxi Journal of Traditional Chinese Medicine, vol. 18, no. 6, p. 242.

Wang, J, Wang, Y \& Yang G 2003, 'Treatment of premenstrual tension syndrome by the combination of massage and acupuncture' (按)摩与针刺结 合治疗经前期紧张综合征), Journal of China-Japan Friendship Hospital, vol. 17, no. 4, p.251.

Wang, J \& Yin, H 2004, Treatment of 120 cases of premenstrual tension syndrome by Jing Qian Kang' (经前康治疗经前期紧张综合征120例), Shandong Journal of Traditional Chinese Medicine, vol. 23, no. 9, pp.524-5.

Wang, K 1602, Standards of Diagnosis and Treatment: Gynecology (证治准 绳.妇科), China.

Wang, M, Hammarback, S, Lindhe, BA \& Backstrom, T 1995, 'Treatment of premenstrual syndrome by spironolactone: a double-blind,

placebo-controlled study', Acta Obstet Gynecol Scand, vol. 74, pp. 803-8.

Wang, R and Zhang, B 1998, 'Treatment of 48 cases of premenstrual oral ulcer by pattern identification' (辨证治疗经期口腔溃疡 48 例), Hunan Guiding Journal of Traditional Chinese Medicine and Pharmacology, vol. 4, no. 12 , p. 23.

Wang, R \& Zhang, Y 2003, 'Treatment of 100 cases of premenstrual tension syndrome by modified Xiao Yao San' (逍遥散加味治疗经前期紧张综合征 100 例), Xinjiang Journal of Traditional Chinese Medicine, vol. 21, no. 3, p. 68. 
Wang, T, Jiang, B \& Zeng, Y 2002, 'Determination of Content of Flavonoids in Single and Mixed Decoction of Four Noble Ingredients' (四君子汤合煎 液与单煎液中总黄酮的测定), Lishizhen Medicine and Maieria Medical Research, vol. 13, no. 9, pp. 519-20.

Wang, W \& Xu, S 1984, ' Conclusion on the treatment of 74 cases of premenstrual breasts distension by Fu Ning Wan’ (妇宁丸治疗经前乳胀 74 例小结), Hubei Zhongyi Zazhi, no. 1, pp. 22-3.

Wang, X, Li, S \& Hu, M 2003, 'Treating premenstrual syndrome by Zhong Xi Medicine in 44 cases' (中西结合治疗经前期综合征 44 例), Chinese Journal of the Practical Chinese with Modern Medicine, vol.16, no. 8, p. 1160 .

Wang, X \& Zheng, C 2002, 'Treatment of 30 cases of premenstrual breasts distension pain by Xiao Yao Wan' (逍遥丸治疗经前乳房胀痛 30 例), Liaoning Journal of Traditional Chinese Medicine, vol. 29, no. 8, p. 484.

Wang, Y 2004, 'Treatment of premenstrual oral ulcer by tonifying both liver and kidney' (肝肾同治阴阳双补法治疗经行口糜), Liaoning Journal of Traditional Chinese Medicine, vol. 31, no. 6, p. 485.

Ward, MW \& Holimon, TD 1999, 'Calcium treatment for premenstrual syndrome', Ann Pharmacother, vol. 33, no. 12, pp. 1356-8.

Williams, MJ, Harris, RI and Dean BC 1985, 'Controlled trial of pyridoxine in the premenstrual syndrome', J Int Med Res, vol. 13, pp. 174-9.

Wiseman, N (translated and amended) 1994, Fundamentals of Chinese medicine, Rev. edn, Paradigm Publications, Brookline, Mass.

Wittchen, HU, Becker, E, Lieb, R \& Krause, P 2002. 'Prevalence, incidence and stability of premenstrual dysphoric disorder in the community', Psychological Medicine, January, vol. 32, pp. 119-132.

Woods, NF, Most, A \& Dery, GK 1982a, 'Estimating perimenstrual distress: a comparison of two methods', Research In Nursing \& Health, vol. 5, no. 2, pp. 81-91.

Woods, NF, Most, A \& Dery, GK 1982b, 'Prevalence of premenstrual symptoms’, Am J Public Health, vol.72, no.11, pp. 1257-64. 
Woods, NF, Lentz, M, Mitchell, E 1986, The daily health diary. The prevalence of perimenstrual symptoms: Final report (NUO1054), Division of Nursing, USPHS, DHHS, Washington D.C.

Woods, NF, Lentz, MJ, Mitchell, ES, Heitkemper, M, Shaver, J \& Henker, R 1998, 'Perceived stress, physiologic stress arousal, and premenstrual symptoms: group differences and intra-individual patterns', Research in nursing \& health, vol. 21, no. 6, pp. 511-23.

World health Organization (WHO) 1979, International Classification of Diseases and Related Health Problems, 9th revision, WHO, Geneva.

World health Organization (WHO) 1992, International Classification of Diseases and Related Health Problems, 10th revision, WHO, Geneva.

Wu, B 1985, 'Clinical experiences of Gu Xiao-chi on the treatment of premenstrual patterns' (顾小痴治疗经前诸证临床经验), Jiangsu Journal of Traditional Chinese Medicine, vol. 6, pp. 3-5.

Wu, H 2001, 'Treatment of 40 cases of premenstrual oral ulcer by Qin Lian Er Di Tang' (芩连二地汤治疗经行口糜 40 例), China's Naturopathy, vol. 9, no. 1, pp. 40-1.

Wu, L \& Tan, Y 2001, 'Discussion on the TCM diagnosis and treatment for peri-menstrual emotional abnormalities' (试论经行情志异常的中医诊治), Journal of Nanjing University of Traditional Chinese Medicine, vol. 17, no. 2, pp. 118-9.

Wu, Q (ed.) 1742, Golden Mirror of Medicine (医宗金鉴), China.

Wu, S \& Zhang, Z 2001, The treatment experience in peri-menstrual vomiting' (经行呕吐治验), Heilongjiang Journal of Traditional Chinese Medicine, no. 4, p. 43.

Wyatt, KM, Dimmock, PW, Jones, PW \& O'Brien, SPM 1999, 'Efficacy of vitamin B-6 in the treatment of premenstrual syndrome: systematic review', $B M J$, vol. 318, pp. 1375-1381. 
Wyatt, K, Dimmock, PW, Jones, P, Obhrai, M \& O'Brien, S 2001, 'Efficacy of progesterone and progestogens in management of premenstrual syndrome: systematic review', BMJ, vol. 323, pp. 776-80.

Xia, G (ed.) 1994, Clinical Gynaecology of Chinese Medicine (中医临床妇 科学), People's Medical Publishing House, Beijing.

Xiao, C 1999, The prevention and treatment of premenstrual tension syndrome (经前期紧张综合征的防治), Family \& Traditional China Medicine, vol. 6, pp. 16-7.

Xiao, X 1689, Classified Treatise on Obstetrics and Gynecology (女科经纶), China.

Xie, Z \& Li, N 1995, 'Methodological Analysis of Clinical Articles on Therapy Evaluation Published in Chinese Journal of Integrated Traditional\& Western Medicine(in Chinese)1982 - 1994', CJIM, vol. 1, no. 4, pp. 301-6.

Xu, S 1982, 'Analysis and discussion on pattern identification and treatment of premenstrual syndrome in 170 cases’ (辨证治疗经前期综合征170例分析 及探讨), Liaoning Journal of Traditional Chinese Medicine, no. 4, pp. 33-4.

$\mathrm{Xu}, \mathrm{X} 2003$, ' Treatment of 60 cases of peri-menstrual swelling by the integration of Chinese and western medicine' (中西医结合治疗经行浮肿60 例), Jiangxi Medical Journal, vol. 38, no. 2, p. 125.

Yan, Y 1999, 'Treatment of 45 cases of premenstrual tension syndrome by modified Xiao Yao San' (逍遥散加味治疗经前期紧张综合征 45 例), Journal of Nanjing University of Traditional Chinese Medicine, vol. 15, no. 2, p. 84.

Yang, J 1993, The pathogenesis, diagnosis and treatment on peri- menstrual symptoms' (月经前后诸症的病机与辨治), Shaanxi Journal of Traditional Chinese Medicine, vol. 14, no. 6, pp. 255-6.

Yang, X 2000, 'Treatment of 60 cases of premenstrual breasts distension pain by Shu Gan Jie Yu Huo Xue Tang' (疏肝解郁活血汤治疗经前乳房 胀痛 60 例), Lishizhen Medicine and Maieria Medical Research, vol. 11, no. 2, p. 162. 
Yang, X \& Zhao, S 2003, 'Treatment of 40 cases of premenstrual tension syndrome by modified Xiao Yao San' (逍遥散加减治疗经前期紧张综合征 40 例), Journal of Practical Traditional Chinese Medicine, vol. 19, no. 3, pp. 124-5.

Yang, Y 2002, 'Treatment of peri-menstrual abdominal pain by Shao Gan Tang in 54cases' (莳甘汤治疗经行腹痛 54 例), Primary Journal of Chinese Materia Medica, $\quad$ vol. 16, no. 2, pp. 59-60.

Yang, Z 2003, 'Treatment of 30 cases of premenstrual headacheby Long Dan Xie Gan Tang' (龙胆泻肝汤治疗经行头痛 30 例), Henan Traditional Chinese Medicine, vol. 23, no. 12, p. 58.

Ye, G 1913, The Complete Book of Ye's Obstetrics and Gynecology (叶氏女 科证治, 叶天士女科全书 或 叶天士女科), China.

Ye, Y 1998, 'Treatment of 48 cases of premenstrual tension syndrome according to pattern identification' (辨证治疗经前期紧张综合征 48 例), Chinese Journal of Information on TCM, vol. 5, no. 4, pp. 33-4.

Yonkers, KA, Clark, RH \& Trivedi, MH 1997, 'The psychopharmacological treatment of nonmajor mood disorders', Modern Problems of Pharmacopsychiatry, vol. 25, pp. 146-66.

Yonkers KA; Pearlstein T; Rosenheck RA Premenstrual disorders: bridging research and clinical reality. Archives Of Women's Mental Health [Arch Womens Ment Health] 2003 Nov; Vol. 6 (4), pp. 287-92.

You, Y 1997, 'Treatment of 56 cases of premenstrual tension syndrome by acupuncture and moxibustion' (针尒治疗经前期紧张综合征 56 例), Chinese Acupuncture and Moxibustion, vol. 17, no. 5, p. 171.

Young, M 2001, 'PMS and PMDD: Identification and treatment', Patient Care, vol. 35, no. 2, pp. 29-30,33-4,37-40.

Yu, J 1997, Combined Chinese-western medicine of clinical obstetric and gynecology (实用中西医结合妇产科学), Beijing Medical University and Chinese Xie He Medical University Joint Publisher, Beijing. 
Yu, J, Liu, B, Liu, Z \& Robinson, V 2005, 'Evaluation of clinical therapeutic effects and safety of acupuncture treatment for premenstrual syndrome' (针 刺治疗经前期综合征的临床疗效和安全性评价), Chinese Acupuncture and Moxibustion, vol. 25, no. 6, pp. 377-82.

Yu, Q \& Liu, Y 2004, 'Observation of treatment effect on 120 cases of premenstrual tension syndrome by Jing Qian An Shu Yin’ (经前安舒饮治疗 经前期紧张症 120 例疗效观察), Yunan Journal of Traditional Chinese Medicine and Materia Medica, vol. 25, no. 4, pp.19-20.

Yuan, R 2002, 'Pattern identification and treatment of premenstrual tension syndrome' (月经前期紧张症的辨证施治), Journal of Inner Mongolia Radio \& TV University, No. 4.

Yue, C 1994, 'Discussion on premenstrual swelling' (经前浮肿证治浅谈), Journal of Beijing University of Traditional Chinese Medicine, vol. 17, no. 4, pp. 23-5.

Yue, X \& Chen, J 2005, 'Clinical research in the treatment of premenstrual syndrome by Xiao Yao San in 300 cases' (逍遥散治疗经前期综合征300例 临床研究), Journal of Practical Medical Techniques, vol. 12, no. 9B, pp. 2572-3.

Zhang, B 1985, Internal Medicine of TCM (中医内科学), Shanghai Science and Technology Press, Shanghai.亚

Zhang, C 1995, 'Observation of the treatment of premenstrual swelling by Dang Gui Shao Yao San in 15 cases'(当归苟药散治疗经前浮肿 15 例观察), Journal of Practical Traditional Chinese Medicine, no. 6, pp. 986-7.

Zhang, E. (ed) 1990a, Basic theory of traditional Chinese medicine, $2^{\text {nd }}$ edn, Publishing House of Shanghai College of TCM, Shanghai

Zhang, E (ed.) 1990b, Diagnostics of traditional Chinese medicine, Publishing House of Shanghai University of TCM, China

Zhang, H 2002, 'Treatment of 92 cases of premenstrual breasts distending pain By the tension syndrome by the combination of acupuncture and auricular point pressing' (针刺结合耳穴贴压治疗经行乳房胀痛 92 例), Shanghai Journal of Acupuncture and Moxibustion, vol. 21, no. 6, pp. 24-5. 
Zhang, H 2003a, 'Treatment of 2 cases of premenstrual breasts distension pain by the combination of Xiao Yao San and Xia Ku Cao paste' (逍遥散加 夏枯草膏治疗经前乳房胀痛 2 例), Modern Medicine Health, vol. 9, no. 10, p. 1234.

Zhang, H 2004a, 'Treatment of 58 cases of premenstrual syndrome by pattern identification' (辨证治疗经前期综合征 58 例), Liaoning Journal of Traditional Chinese Medicine, vol. 31, no. 3, p. 224.

Zhang, H \& Chen, Y 1998, 'Treatment of 30 cases of premenstrual headache by Xue Fu Zhu Yu Tang' (血府逐癖汤加减治疗经行头痛 30 例), Journal of Anhui Traditional Chinese Medical College, vol.17, no. 6, pp. 18-9.

Zhang, HL 2003b, 'Treatment of 19 cases of premenstrual diarrhoea by You Gui Wan’ (右归丸治疗经行泄泻19例), Sichuan Journal of Traditional Chinese Medicine, vol. 21, no. 5, p. 53.

Zhang, J \& Sun, X 2003, 'Clinical observation of treatment of premenstrual tension syndrome by the integration of Chinese and western medicine in 84 cases' (中西医结合治疗经前期紧张综合征84例疗效观察), Shandong Medical Journal, vol. 43, no. 7, p. 26.

Zhang, L 1695, Zhang's Treatise on General Medicine (张氏医通), China.

Zhang, L 2005, 'Premenstrual syndrome and liver failing to disperse and purge’ (经前期综合征与肝失疏泄), Jiangxi Journal of Traditional Chinese Medicine, vol. 36, no. 267, pp. 20-1.

Zhang, L \& Chen, L 2000, 'Treatment of 50 cases of premenstrual tension syndrome by the combination of acupuncture, moxibustion and Chinese medicine' (针尒中药并用治疗经前期紧张症 50 例), Ningxia Medical Journal, vol. 22, no. 7, p. 436.

Zhang, N 2004b, 'Treatment of 36 cases of premenstrual tension syndrome by modified Dang Gui Shao Yao San” (当归药药散加味治疗经前期紧张综合 征36例), Shaanxi Journal of Traditional Chinese Medicine, vol. 25, no. 11, pp. 986-7.

Zhang, Q 2003c, 'Treatment of 64 cases of premenstrual syndrome by TCM cycle therapy’ (中药周期疗法治疗经前期综合征64例), Shaanxi Journal of Traditional Chinese Medicine, vol. 24, no. 5, pp. 971-2. 
Zhang, R 2003d, 'Clinical observation of treatment of premenstrual syndrome by massage' (按摩治疗经前期综合征临床观察), Chinese Manipulation \& Qi Gong Therapy, vo1. 19, no. 2, pp. 49-50.

Zhang, X \& Li, Z 2004, Treating peri-menstrual diarrhoea by modified Shen Ling Bai Zhu San' (参苓白术散加味治疗经行泄泻), Shanxi Journal of Traditional Chinese Medicine, vol. 20, no. 2, p. 17.

Zhao, C, Zhao, M, Zhao, T \& Wu, X 2000, 'Treatment of 55 cases of premenstrual feverish sensation by Fu Fe Qian Jin Pian’ (妇科千金片治疗 经行发热 55 例), Hunan Zhongyi Zazhi, vol. 16, no. 4, p. 41.

Zhao, G, Wang, L \& Qu, C 1998, 'Prevalence of premenstrual syndrome in reproductive women and its influential factors’ (育齡婦女經前期綜合征的發 生情況及影響因素), Chin J Obstet Gynecol, vol. 33, no. 4, pp. 222-24.

Zhao, Y, Li, C \& Li M 2005, 'Treatment of 58 cases of premenstrual breasts distension pain by liver coursing, stagnation resolving and blood moving method' (疏肝解郁活血法治疗经前乳房胀痛58例), Occupation and Health, vol. 21 , no. 10 , p. 162 .

Zheng, G, Zhao, A \& Zhai, F 2002, 'Treatment of 100 cases of premenstrual syndrome by the combination of Xiao Yao San and auricular point pressing' (逍遥散配合耳穴压贴法治疗经前期综合征 100 例), China' $s$

Naturopathy, vol. 10, no. 8, pp. 46-7.

Zhong, J \& Chen, Y 2001, 'Treating premenstrual headache through applying treatment to liver' (经行头痛从肝论治), New Journal of Traditional Chinese Medicine, vol. 33, no. 3, p. 70.

Zhou, L \& Cao, L 2000, 'Mediation Method Is the Key Therapy for Menstruous Fever' (经行发热 和解为要), Shanghai Journal of Traditional Chinese Medicine, no. 6, pp. 26-7.

Zhou Y 2002, 'Clinical observation of treatment of premenstrual breasts distension pain in 43 cases' (滋水涵木法治疗经前乳房胀痛 96 例临床观 察), Gansu Journal of Traditional Chinese Medicine, vol. 15, no. 4, pp. 69-70. 
Zhou, Y-m 1999 , 'Observation of treatment effect on 53 cases of premenstrual headache by Juan Tong Yin' (湴蜀痛饮”治疗经行头痛 53 例疗 效观察), Journal of Guiyang College of Traditional Chinese Medicine, vol. 21 , no. 4 , p. 25.

Zhou, YM, Zhao, X \& Sun, X 2002, 'Treating 26 cases of peri-menstrual rashes by Chinese medicine' (中药治疗月经疹 26 例), New Journal of Traditional Chinese Medicine, vol. 34, no. 1, p. 55.

Zhou, Z 2003, 'Using the strategies of coursing liver and tonifying kidney as basic treatment for premenstrual syndrome' (疏肝补肾法为主治疗经前期 综合征), Henan Traditional Chinese Medicine, vol. 23, no. 12, p. 49.

Zhou, Z, \& Zhu, X 1984, 'A clinical study of qi and blood patterns identification in hearts diseases and $\mathrm{TXB}_{2}$ 6-keto-PGF a'(心病气血辨证与 $\mathrm{TXB}_{2}$ 6-keto-PGF a 的临床研究), China Journal of Basic Medicine in Traditional Chinese Medicine, vol. 4, no. 4, p.30.

Zhu, H 2001, 'Treating 42 cases of peri-menstrual headache by self designed formula' (自拟中药方治疗经行头痛42例), Sichuan Journal of Traditional Chinese Medicine, vol. 19, no. 9, p. 52.

Zhu, M \& Xu, X 2003, Clinical research in the treatment of premenstrual tension syndrome by liver coursing and spirit calming method' (疏肝安神法 治疗经前期紧张综合征的临床研究), China Journal of Traditional Chinese Medicine and Pharmacy, vol. 18, no. 5, pp. 286-8.

Zoldan, VA, Pereira, ME \& Tarelho, LC 2004, 'A psychoanalytical approach to premenstrual tension syndrome', Psychoanalytic review (Brazil), vol. 91, no. 1 , pp. $45-69$. 
Appendix A

Guidelines for Clinical Research in New TCM Medicines for Pre-menstrual syndrome, Ministry of Health, People's Republic of China

Premenstrual syndrome refers to a collection of symptoms e.g. headache, sore and distended breasts, fatigue, tension, depression or agitation, irritability, insomnia, abdominal pain, swelling. These symptoms appear 7-14 days before menstruation, and disappear naturally after the menstruation.

Diagnosis Standard

1. Western Medicine Clinical Diagnosis Standard

(1) General fatigue, tiredness, anxiety, depression, irritability, insomnia, headache, sore and distended breasts, sore back and dragging feeling in the pelvic, swelling, weight gain, and gastrointestinal dysfunction appear 7-14 days before menstruation, and last until menstruation begin

(2) Basal body temperature indicates short luteal phase, slow or irregular rise after ovulation.

(3) Hormonal test indicates high than normal estrodiol level or high ratio between estrodiol and progesterone,

\section{TCM Pattern Identification}

Liver stagnation transforming into Heat:

Sore breasts, distended breasts, hypochondrium distended pain, nausea, vomiting, depression, agitation, bitter mouth, dizziness, red tongue with scanty coating, wiry and slippery pulses appear at premenstrual period.

$Q i$ and Blood stagnation:

Migraine, sore and distended breasts, lower abdominal pain and distension that dislike pressure, dark and purplish menses with clots, dull tongue or stasis at the tip or sides of the tongue, thin and choppy or thin and wiry pulses appear at premenstrual period.

Yin deficiency with excessive Yang 
Headache, dizziness, agitation, irritability, insomnia, sore back and knees, warm palms and soles, bitter mouth, dry throat, constipation, red tongue with yellowish coating, thin and wiry pulses appear at premenstrual period.

Yin deficiency with Hyperactive Fire

Distended and itchy breasts, depression, crying, palpitation, insomnia, difficulty concentrating, dry mouth and tongue, oral ulcer, red tongue tip with scanty coating, thin and rapid pulses appear at premenstrual period.

Spleen and Kidney Yang deficiency

Facial and lower limbs swelling, loose stool, abdominal distension, tired and weakness of limbs, aversion to cold, cold limbs, pale and fresh tongue with thin white coating, soft and moderate or deep and slow pulses appear at premenstrual and menstrual period

Grading of severity

Mild: a low degree of headache, dizziness, and distended breasts; or mild pain, facial swelling; work and study as normal days.

Moderate: dizziness, headache, sore and distended breasts, irritability, insomnia, tension or depression, swelling limbs, symptoms do not affect work or study.

Severe: acute headache, dizziness, fuzzy vision, palpitation, insomnia, distended and sore breasts, pitted oedema over face, limbs or whole body, weight gain 1-2 kg, symptoms disrupt work and study. 
Appendix B

The six peri-menstrual symptoms in the Criteria of Diagnosis and Therapeutic Effect of Gynaenecologic Diseases and Syndromes in

Traditional Chinese Medicine in the Professional Standards of Chinese Medicine, the Republic of China ZY/T 001.3-94

Peri-menstrual breast distension:

Diagnosis criteria:

- Distended pain over breasts and/nipples, usually appears one week before menstruation or during menstruation, disappear after menstruation and is cyclical.

- Exclude the breast pain which is caused by substantial mass of the breast.

Pattern Identification:

Liver $Q i$ stagnation:- hard and distended pain of breasts before or during menstruation; or distended pain and itchiness of nipples, depression, angry, unhappy, thin and white tongue coating and a wiry pulse.

Liver stagnation with Kidney deficiency:- breast distension before or during menstruation, not lump felt on palpation, resolve after menstruation, sore back, tinnitus, 5 hearts heat, red tongue with scant coating, thin and rapid pulses.

Outcome:

- Full recovery: disappearance of breasts pain and distension, no cyclical attack

- Improvement: reduction in breasts pain and distension, or disappearance of symptoms but recurrence within three months.

- $\quad$ No effect: peri-menstrual breasts pain and distension remain unchanged.

\section{Peri-menstrual fever/feverish sensation:}

Diagnosis criteria:

- Fever/feverish sensation before, during and after menstruation, it appears cyclically and more than two times. 
- Distinguish with the common flu during menstruation time.

Pattern identification:

Yin deficiency with interior Heat:-

Fever/feverish sensation before and after menstruation, tidal fever in the afternoon, agitated, insomnia, dry mouth, constipation, dry and red tongue, thin and rapid pulses.

Qi and Blood deficiency:-

Fever/feverish sensation during or after menstruation, excessive and pale coloured period, tiredness, aversion to coldness, shortness of breath, reluctant to speak, swelling face, pale and fresh tongue with white and moist coating, a deficient pulse or thin and slight rapid pulses.

Disharmony between Ying and Wei:-

Fever/feverish sensation during menstruation, scant and sluggish period, lower abdominal distension, aversion to wind, spontaneous sweating, pale red tongue with thin and white coating, and floating and moderate pulses.

Interior Heat stagnation:-

Fever/feverish sensation before or during menstruation, dark and dull period, blood clots in period occasionally, abdominal pain, gloomy tongue or stasis specks on the tip and sides of the tongue, deep, wiry and rapid pulses.

\section{Outcome:}

- Full recovery: disappearance of fever/feverish sensation, no cyclical attack

- Improvement: reduction in fever/feverish sensation reduced, or disappearance of symptoms but recurrence within three months.

- $\quad$ No effect: peri-menstrual fever/feverish sensation remain unchanged.

\section{Peri-menstrual headache}

Diagnosis criteria:

- Headache appears regularly with menstrual cycle over two times.

- Headache usually is on one side of the head, maybe on the temples or top of the head, stabbing or detention pain, or pain continuously.

- Distinguish with headaches due to flu during menstruation, hypertension and intra-cranial space-occupying lesion. 
Pattern identification:

$Q i$ and Blood stagnation:-

Severe headache before or during menstruation, stabbing pain, scant and sluggish period, purplish coloured period with clots or with lower abdominal pain that dislikes touching, dull tongue with stasis spots, thin and choppy pulses or wiry and choppy pulses.

Liver Blood deficiency:-

Headache continuously during or after menstruation, palpitation, insomnia, tired, lethargic, a pale tongue with thin coating, and a thin pulse.

Exuberance of Liver Fire:-

Headache and distended eyes before menstruation, or severe detention pain on the top of the head, agitated, irritability, bitter mouth, dry throat, heavy and fresh period, red tongue with thin and white coating, wiry and rapid pulses.

Outcome:

- Full recovery: disappearance of headache, no cyclical attack

- Improvement: reduction in headache, or disappearance of symptoms but recurrence within three months.

- $\quad$ No effect: peri-menstrual headache remain unchanged.

\section{Peri-menstrual bodyache}

Diagnosis criteria:

- Body ache/soreness or numbness over body and limbs before, during, and after menstruation, it happens cyclically and more than two times.

- No localized swelling or redness, no abnormal gait, no severe external injury, arthritis or other medical history

Pattern identification:

Qi and Blood deficiency:-

Body ache/soreness or numbness over body and limbs before, during, and after menstruation, tired, lethargic, scant and pale period, pale tongue with thin coating, and thin pulse.

Cold and Damp stagnation:-

Pain, soreness and heaviness sensation over joints before, during and after menstruation, especially over knee joints, better with warmth, late 
menstruation, scant, sluggish and dull period with clots, thin white tongue coating, deep and tight pulses.

Outcome:

- Full recovery: disappearance of bodyache, no cyclical attack

- Improvement: reduction in bodyache, or disappearance of symptoms but recurrence within three months.

- $\quad$ No effect: peri-menstrual bodyache remain unchanged.

$\underline{\text { Peri-menstrual diarrhoea }}$

Diagnosis criteria:

- Diarrhea appears during menstruation following the menstrual cycles.

- Loose stool or stool with undigested food, usually no abdominal pain, stool has no odour, pus or blood.

- Distinguish with chronic diarrhea.

Pattern identification:

Liver stagnation with Spleen deficiency:-

Loose stool during menstruation, tired, heavy limbs, lusterless complexion, poor appetite, bland mouth, or abdominal distension with vague pain, pale tongue with thin coating and teeth marks, soggy pulse. If condition also has exuberant Liver, there will be lower abdominal distension, chest and hypochondriac oppression, soggy and wiry pulses.

Kidney Yang deficiency:-

Diarrhoea or stools with undigested food during menstruation, dull complexion, sore and weak back and legs, cold physique and limbs, clear and long urination, pale tongue with white and slippery coating, deep and thin pulses.

\section{Outcome:}

- Full recovery: disappearance of diarrhoea, no cyclical attack

- Improvement: reduction in diarrhoea, or disappearance of symptoms but recurrence within three months.

- $\quad$ No effect: peri-menstrual diarrhoea remain unchanged. 


\section{$\underline{\text { Peri-menstrual oral ulcer }}$}

Diagnosis criteria:

- Oral ulcer appears within one week before menstruation, during menstruation, or within 3-4 days after menstruation, it appears cyclically.

- Normal result on gynecological and ophthalmological examination, exclude $\mathrm{Hu} \mathrm{Huo} \mathrm{disease.}$

Pattern identification:

Yin deficiency with exuberant Fire:-

Oral ulcer during menstruation, dry mouth and throat, 5 hearts heat, emaciation, dizziness, sore back, insomnia, scant and yellow urination, red tongue tip, thin and rapid pulses.

Exuberance of Stomach Heat:-

Oral ulcer during menstruation, halitosis, dry mouth, desire for drinks, chest oppression, poor appetite, yellow urine, constipation, yellow and thick tongue coating, slippery and rapid pulses.

Outcome:

- Full recovery: disappearance of oral ulcer, no cyclical attack

- Improvement: reduction in oral ulcer, or disappearance of symptoms but recurrence within three months.

- $\quad$ No effect: peri-menstrual oral ulcer remain unchanged. 


\section{Appendix C}

\section{Reference List of the 124 Chinese Clinical Trials 1996-2005}

\begin{tabular}{|c|c|c|c|c|c|}
\hline No & Code & Title & Author & Source & Date \\
\hline 1 & Pu32 & 逍遥散治疗经前期综合征 300 例临床㸴究 & 岳秀娟 & 实用医技杂志 & $2005 / 18$ \\
\hline 2 & YE29 & $\begin{array}{l}\text { 逍遥散加减治疗肝郁气滞型经前乳房胀痛 } 36 \text { 例疗效 } \\
\text { 观察 }\end{array}$ & 冯长柏 & 河北中医 & $2005 / 10$ \\
\hline 3 & Pu3 & 丹柧逍遥散加减治疗经前期综合征 21 例 & 陈敏 & 实用中医药杂志 & $2005 / 02$ \\
\hline 4 & Pu20 & 芎芷石膏汤加减治疗经行头痛 50 例 & 蔡建文 & 新中医 & $2005 / 03$ \\
\hline 5 & YE30 & 健脾疏肝法治疗经前期综合症 24 例 & 杨培俐 & 现代医药卫生 & $2004 / 24$ \\
\hline 6 & Pu6 & 当归苟药散加味治疗经前期紧张综合征 36 例 & 张宁海 & 陕西中医 & $2004 / 11$ \\
\hline 7 & 030 & 经前康治疗经前期紧张综合征 120 例 & 王君 & 山东中医杂志 & $2004 / 09$ \\
\hline 8 & Ye14 & 辨证治疗经前期综合征 58 例 & 张慧珍 & 辽宁中医杂志 & $2004 / 03$ \\
\hline 9 & 062 & 龙胆泻肝汤加减治疗经前期痤疮 84 例 & 姜永珊 & 新中医 & $2003 / 09$ \\
\hline 10 & 044 & 经前舒液治疗经前期综合征 50 例分析 & 李仲本 & 中医药学刊 & $2003 / 10$ \\
\hline 11 & 053 & 逍遥散加减治疗经前期紧张综合征 40 例 & 杨晓红 & 实用中医药杂志 & $2003 / 03$ \\
\hline 12 & 054 & 逍遥散加减治疗经前期综合症 46 例疗效观察 & 高宗丽 & 云南中医中药杂志 & $2003 / 03$ \\
\hline 13 & 029 & 经前平颗粒治疗经前期综合征 60 例临床体会 & 王优快 & 实用临床医药杂志 & $2003 / 01$ \\
\hline 14 & 057 & 逍遥散加味治疗经前期紧张综合征 100 例 & 王荣华 & 新疆中医药 & $2003 / 03$ \\
\hline 15 & 056 & 逍遥散加味治疗月经前期综合征 86 例 & 傅红 & 实用中西医结合临床 & $2003 / 04$ \\
\hline 16 & 021 & 疏肝补肾法为主治疗经前期综合征 & 周征 & 河南中医 & $2003 / 12$ \\
\hline 17 & 019 & 疏肝安神法治疗经前期紧张综合征的临床硏究 & 朱梅 & 中国医药学报 & $2003 / 05$ \\
\hline 18 & Pu14 & 血府逐瘀胶囊治疗经前期紧张综合征 52 例 & 陈珊 & 北京中医 & $2003 / 06$ \\
\hline 19 & 010 & 加味龙胆泻肝汤治疗经前期头目胀痛 78 例 & 邱明英 & 新中医 & $2003 / 04$ \\
\hline 20 & 05 & 中药治疗经前偏头痛 48 例 & 孙宏丽 & 新中医 & $2003 / 02$ \\
\hline 21 & 028 & 经前平颗粒治疗经前期紧张综合征 82 例临床观察 & 黄巧红 & 江苏临床医学杂志 & $2002 / 04$ \\
\hline 22 & G3 & $\begin{array}{l}\text { 解有消胀煎治疗经前期综合征 } 400 \text { 例——附西药对 } \\
\text { 照组 } 200 \text { 例 }\end{array}$ & 李传香 & 辽宁中医杂志 & $2002 / 04$ \\
\hline 23 & 022 & 疏肝解有健脾法治疗经前期综合征 39 例 & 王斌 & 福建中医药 & $2002 / 04$ \\
\hline 24 & B4 & $\begin{array}{l}\text { 经前平颗粒多中心 、随机双亘双模拟对照治疗经前 } \\
\text { 期综合征肝气逆证 } 403 \text { 例 }\end{array}$ & 乔明琦 & 中国新药杂志 & $2002 / 05$ \\
\hline 25 & YE32 & 中药周期疗法治疗经前期综合征 64 例 & 张琪 & 陕西中医 & $2002 / 11$ \\
\hline 26 & G9 & 舒肝宁神汤治疗经前期紧张综合征 68 例 & 丁树栋 & 中医药硏究 & $2001 / 03$ \\
\hline 27 & G17 & 辨证治疗经前期综合征 188 例观察 & 陈文英 & 实用中医药杂志 & $2001 / 01$ \\
\hline 28 & G15 & $\begin{array}{l}\text { 贯叶连朝合剂治疗经前期紧张综合征 }-55 \text { 例临床资 } \\
\text { 料分析 }\end{array}$ & 曾真 & 中成药 & $2001 / 02$ \\
\hline 29 & G35 & 疏肝调经法治疗经前期紧张综合征 & 王金牛 & 江西中医药 & $2000 / 06$ \\
\hline 30 & P3 & 覓遥散加味治疗经前期紧张综合征 45 例 & 殷燕云 & $\begin{array}{l}\text { 南京中医药大学学报 (自 } \\
\text { 然科学版) }\end{array}$ & $1999 / 02$ \\
\hline 31 & R5 & 坤月宁治疗痛经及经前期综目征的临床及实验㸴究 & 黄玲 & 现代中西医结合杂志 & $1999 / 05$ \\
\hline
\end{tabular}




\begin{tabular}{|c|c|c|c|c|c|}
\hline 32 & G40 & “助阳解有汤” 治疗经前期紧张综合征 32 例 & 钱菁 & 江苏中医 & 1999/12 \\
\hline 33 & G42 & 疏肝健脾汤治疗经前期紧张综合征 58 例 & 李霞 & 安徽中医学院学报 & $1999 / 04$ \\
\hline 34 & R44 & 辨证治疗经前期紧张综合征 48 例 & 叶艺玲 & 中国中医药信息杂志 & 1998/04 \\
\hline 35 & P5 & 从湿论治经前头痛 40 例 & 李琼 & 新中医 & $1998 / 12$ \\
\hline 36 & R66 & 疏肝法治疗经前期综合征 38 例观察 & 蔡慧筠 & 实用中医药杂志 & $1997 / 05$ \\
\hline 37 & P20 & 逍遥散加减治疗经前期紧张综合征 62 例 & 王君 & 陕西中医 & $1997 / 06$ \\
\hline 38 & R64 & 经前安泰汤治疗经前期紧张综合征 228 例 & 傅赛萍 & 陕西中医 & $1997 / 06$ \\
\hline 39 & P25 & 中医治疗经前期紧张综合征 60 例临床疗效观察 & 梁香云 & 云南中医中药杂志 & $1996 / 05$ \\
\hline 40 & P30 & 逍遥丸治疗经前期紧张综合征 & 史冬梅 & 时珍国医国药 & $1996 / 01$ \\
\hline 41 & YE35 & 滋肾疏肝法对经前期紧张综合征的疗效观察 & 冯家阳 & 四川中医 & $1996 / 02$ \\
\hline 42 & P29 & 舒肝解有活血化瘀法治疗经前期躁狂抑有症 30 例 & 陈宗光 & 新中医 & $1996 / 03$ \\
\hline 43 & YE36 & 疏肝健脾法治疗经前乳胀 69 例疗效观察 & 吴霆 & 中医药信息 & $2005 / 05$ \\
\hline 44 & 025 & 经前安舒饮治疗经前期紧张症 120 例疗效观察 & 于庆民 & 云南中医中药杂志 & $2004 / 04$ \\
\hline 45 & 06 & 中药治疗经前偏头痛 & 穆翠英 & 山西中医 & $2004 / 03$ \\
\hline 46 & 047 & 自拟疏肝通络汤治疗经前乳痛症 50 例 & 毕华 & 浙江中医杂志 & $2004 / 01$ \\
\hline 47 & 048 & 血府逐疼汤治疗经前失眠症 127 例 & 陈已明 & 陕西中医 & $2003 / 05$ \\
\hline 48 & 051 & 逍遥丸治疗经前乳房胀痛 30 例 & 王晓艳 & 辽宁中医杂志 & $2002 / 08$ \\
\hline 49 & 018 & 滋水涵木法治疗经前乳房胀痛 96 例临床观察 & 周亚红 & 甘肃中医 & $2002 / 04$ \\
\hline 50 & G20 & 经前舒汤治疗经前期紧张症 52 例 & 陆启滨 & 云南中医中药杂志 & $2001 / 04$ \\
\hline 51 & YE37 & 舒肝解郁 健脾和胃法治疗经前乳胀 30 例 & 刘冬梅 & 河北中医药学报 & $2001 / 02$ \\
\hline 52 & G18 & 滋肾水养肺阴治疗经前乳房胀痛 30 例 & 朱利群 & 新中医 & $2001 / 01$ \\
\hline 53 & G26 & 妇乐口服液治疗经前乳房胀痛 480 例 & 倪守涛 & 中国中医药科技 & $2000 / 04$ \\
\hline 54 & G30 & 消胀饮治疗经前乳胀 86 例 & 李蕴 & 河北中医 & $2000 / 05$ \\
\hline 55 & YE28 & 自拟解有胺神汤治疗经前浮肿 32 例 & 李鲁炎 & 吉林中医药 & 1999/02 \\
\hline 56 & G44 & 疏肝活血冲剂治疗经前口疮 43 例临床观察 & 谭劲 & 中国中医药科技 & 1999/02 \\
\hline 57 & YE40 & 中药治疗经行头痛 60 例 & 于水永 & 光明中医 & $2005 / 06$ \\
\hline 58 & PU22 & 肝肾同治阴阳双补法治疗经行口糜 & 王艳霞 & 辽宁中医杂志 & $2004 / 06$ \\
\hline 59 & YE3 & 参苓白术散加味治疗经行泄泻 & 张霞 & 山西中医 & $2004 / 02$ \\
\hline 60 & YE42 & 四物汤加味治疗肝血虚型经行头痛 72 例疗效观察 & 蔡屏江 & 云南中医中药杂志 & $2003 / 06$ \\
\hline 61 & YE43 & 龙胆泻肝汤治疗经行头痛 30 例 & 杨准叶 & 河南中医 & $2003 / 12$ \\
\hline 62 & PU18 & 中药治疗经行头痛 & 夏隽晖 & 湖北中医杂志 & $2003 / 07$ \\
\hline 63 & PU29 & 右归丸治疗经行泄泻 19 例 & 张宏亮 & 四川中医 & $2003 / 05$ \\
\hline 64 & B12 & 中医药治疗经行头痛 108 例 & 连家成 & 湖北中医杂志 & $2002 / 01$ \\
\hline 65 & YE44 & 自拟中药方治疗经行头痛 42 例 & 朱惠莲 & 四川中医 & $2001 / 09$ \\
\hline 66 & YE45 & 辨证治疗经行乳房胀痛 32 例临床观察 & 王丽珠 & 云南中医中药杂志 & $2000 / 05$ \\
\hline 67 & YE46 & 辨证治疗经行咳嗽 30 例的体会 & 徐丽英 & 云南中医中药杂志 & $2000 / 01$ \\
\hline 68 & YE47 & 衍液宝合剂治疗经行发热 166 例 & 韩交刚 & 河南中医 & $2000 / 02$ \\
\hline
\end{tabular}




\begin{tabular}{|c|c|c|c|c|c|}
\hline 69 & YE48 & 妇科千金片治疗经行发热 55 例（三等奖） & 赵椰 & 湖南中医杂志 & $2000 / 04$ \\
\hline 70 & YE49 & 头痛宁治疗经行头痛 52 例 & 翁广兰 & 中医硎究 & $1999 / 04$ \\
\hline 71 & YE50 & 辨病辨证治疗经行头痛 35 例 & 杨丽珠 & 云南中医中药杂志 & $1999 / 03$ \\
\hline 72 & YE51 & 加味四物汤治疗经行菖麻疹 9 例 & 赵景明 & 山西中医 & $1999 / 03$ \\
\hline 73 & YE54 & 辨证论治经行乳房胀痛 30 例 & 胡荣桂 & 湖北中医杂志 & $1999 / 04$ \\
\hline 74 & YE56 & “蠋痛饮” 治疗经行头痛 53 例疗效观察 & 周易明 & 新疆中医药 & $1999 / 04$ \\
\hline 75 & YE57 & 四物玉屏汤加味治疗经行风疹块 & 张秋枫 & 山东中医杂志 & $1999 / 07$ \\
\hline 76 & YE58 & 二地芩连饮治疗经行口糜 40 例 & 莫璐丽 & 新中医 & $1999 / 12$ \\
\hline 77 & YE59 & 中药治疗经行身痛的临床观察 & 姜坤 & 中国中医基础医学杂志 & $1998 / \mathrm{S} 1$ \\
\hline 78 & YE60 & 理中汤加味治疗经行吐泻 & 赵素荵 & 辽宁中医杂志 & $1998 / 08$ \\
\hline 79 & YE61 & 血府逐瘀汤加减治疗经行头痛 30 例 & 张华玉 & 安徽中医学院学报 & $1998 / 06$ \\
\hline 80 & YE62 & “消痛汤” 治疗经行头痛 86 例 & 滕月新 & 江苏中医 & $1997 / 01$ \\
\hline 81 & YE63 & 372 例经行感冒的中医诊疗初探 & 沈家骥 & 云南中医学院学报 & $1996 / 01$ \\
\hline 82 & YE64 & 大柴胡汤加减治疗经行咽喉肿痛 66 例 & 张长义 & 山东中医杂志 & $1996 / 04$ \\
\hline 83 & YE65 & 桃核承气汤加味治疗经行发热 80 例 & 王广见 & 山东中医杂志 & $1996 / 01$ \\
\hline 84 & YE67 & 辨证分型治疗经行口舌糜烂 40 例小结 & 周征 & 中医药导报 & $2005 / 07$ \\
\hline 85 & PU25 & 小柴胡汤加减治疗经行发热 18 例 & 林艺杰 & 实用中医药杂志 & $2005 / 06$ \\
\hline 86 & YE68 & 调经助孕冲剂治疗经行乳房胀痛 54 例 & 张晓冬 & 中医药信息 & $2004 / 01$ \\
\hline 87 & YE69 & 疏肝调经法治疗经行口糜 60 例 & 张红香 & 黑龙江中医药 & $2003 / 01$ \\
\hline 88 & YE70 & 自拟二仙姜独汤治疗经行身痛 86 例 & 叶金芳 & 中国中医药科技 & $2002 / 01$ \\
\hline 89 & YE71 & 疏肝养肝法治疗经行头痛 40 例 & 李秀华 & 福建中医药 & $2002 / 01$ \\
\hline 90 & YE72 & 桂枝汤加味治疗经行头痛 36 例 & 朱峪英 & 广西中医药 & $2001 / 01$ \\
\hline 91 & YE4 & 参苓白术散治疗经行泄泻 24 例 & 李淑平 & 吉林中医药 & $2000 / 01$ \\
\hline 92 & YE73 & 健固汤治疗经行泄泻 38 例临床观察 & 杨慧珊 & 江西中医药 & $2000 / 05$ \\
\hline 93 & YE74 & 一贯煎治疗经行头痛 148 例 & 韩风云 & 黑龙江中医药 & $1996 / 06$ \\
\hline 94 & YE75 & 加味玉女煎治疗经行口糜 38 例 & 陶佩君 & 上海中医药杂志 & $1996 / 12$ \\
\hline 95 & YE76 & 疏肝解有活血汤治疗经前乳房胀痛 60 例 & 杨晓翡 & 时珍国医国药 & $2000 / 02$ \\
\hline 96 & PU38 & 柴胡桂枝汤防治经行感氞 63 例 & 林丹 & 海南医学院学报 & $2003 / 09$ \\
\hline 97 & Pu39 & 丹㖃逍遥散加减治疗经前期综合症 58 例 & 窦荣华 & 国医论坛 & $2004 / 09$ \\
\hline 98 & $\mathrm{Pu} 40$ & 清肝活血颗粒剂治疗经前期痤疮 89 例 & 林欢儿 & 河北中医 & $2004 / 07$ \\
\hline 99 & Pu41 & 加减散偏汤治疗育龄期妇女经前头痛 113 例 & 张志兰 & 中国中医药信息杂志 & $2002 / 11$ \\
\hline 100 & $\mathrm{Pu} 42$ & 中药治疗经前期综合症 78 例小结 & 马志荣 & 贵阳医学院学报 & $2004 / 04$ \\
\hline 101 & $\mathrm{Pu} 43$ & 养阴清热调冲任治疗经前期痤疮 36 例 & 吕海香 & 广西中医药 & $2004 / 02$ \\
\hline 102 & $\mathrm{Pu} 44$ & 养血柔肝息风通络法治疗经前期偏头痛 48 例 & 曹文 & 河北中医 & $2003 / 08$ \\
\hline 103 & Pu45 & 除痤疡治疗经前面部痤疮 32 例 & 刘德彬 & 山东中医杂志 & $2002 / 03$ \\
\hline 104 & PU46 & 抗脑衰胶囊治疗经前紧张综合征 48 例观察 & 梁韶春 & 医学理论与实践 & $2002 / 03$ \\
\hline 105 & PU47 & 舒肝解有健脾和胃法治疗经前乳胀 30 例 & 刘冬梅 & 河北中医药学报 & $2001 / 16$ \\
\hline
\end{tabular}




\begin{tabular}{|c|c|c|c|c|c|}
\hline 106 & PU21 & 疏肝解有活血法治疗经前乳房胀痛 58 例 & 赵迎春 & 职业与健康 & $2005 / 10$ \\
\hline 107 & YE2 & 从肝失疏泄论治经前期紧张症 60 例疗效观察 & 唐华伟 & 云南中医中药杂志 & $2005 / 03$ \\
\hline 108 & YE13 & 苓桂术甘汤治疗经行浮肿 46 例 & 刘承伟 & 吉林中医药 & $2000 / 03$ \\
\hline 109 & YE21 & 辨证治疗经期头痛 30 例 & 黄美珍 & 四川中医 & $2000 / 07$ \\
\hline 110 & YE22 & 川芎汤治疗经前头痛 105 例临床观察 & 曹宁芳 & 陕西中医学院学报 & $2001 / 05$ \\
\hline 111 & YE23 & 调肝通经汤治疗月经期偏头痛 32 例 & 李云慧 & 河北中医 & $2000 / 09$ \\
\hline 112 & YE24 & 自拟芩知饮治疗经行口糜 42 例 & 高丽萍 & 福建中医药 & $2001 / 06$ \\
\hline 113 & YE25 & 芩连二地汤治疗经行口糜 40 例 & 胡慧寅 & 中国民间疗法 & $2001 / 01$ \\
\hline 114 & YE26 & 归脾汤治疗经行情志异常 36 例体会 & 华占海 & 甘肃中医 & $2000 / 02$ \\
\hline 115 & YE27 & 当归药药散治疗经前浮肿 15 例观察 & 张春荣 & 实用中医药杂志 & $1995 / 06$ \\
\hline 116 & 020 & 疏肝散治疗经前乳胀 76 例报告 & 田萍 & 山东医药 & $2003 / 28$ \\
\hline 117 & 063 & $\begin{array}{l}\text { 当归四逆汤治疗经期头痛 } 100 \text { 例——附头痛片治疗 } \\
30 \text { 例对照 }\end{array}$ & 林珍莲 & 浙江中医杂志 & $2000 / 10$ \\
\hline 118 & 064 & 中药治疗月经疹 26 例 & 周玉梅 & 新中医 & $2002 / 01$ \\
\hline 119 & G41 & 自拟栝萎散治疗经前乳房胀痛 233 例 & 李德新 & 四川中医 & 1999/11 \\
\hline 120 & P2 & 疏肝解有汤治疗经前乳胀症 60 例 & 高樟娥 & 浙江中医杂志 & 1999/03 \\
\hline 121 & P6 & 滋阴养血法治疗经前期紧张综合症 154 例 & 韩风云 & 黑龙江中医药 & $1998 / 08$ \\
\hline 122 & $\mathrm{P} 42$ & 芪附四君汤治疗经前期紧张综合征50例 & 黎济民 & 湖北中医杂志 & 1998/02 \\
\hline 123 & P28 & 疏肝逐瘀汤治疗经前乳房胀痛 45 例 & 梁彩云 & 河北中医 & 1996/03 \\
\hline 124 & R9 & 逍遙散加減治療經期諸症 200 例 & 林名宽 & 陝西中医 & 1999/05 \\
\hline
\end{tabular}


Appendix D

Randomisation Table

\begin{tabular}{|c|l|l|l|l|l|l|l|l|l|l|l|l|l|l|l|l|l|l|l|l|l|l|l|l|l|l|l|l|l|l|l|}
\hline Pattern & 1 & 2 & 3 & 4 & 5 & 6 & 7 & 8 & 9 & 1 & 1 & 1 & 1 & 1 & 1 & 1 & 1 & 1 & 1 & 2 & 2 & 2 & 2 & 2 & 2 & 2 & 2 & 2 & 2 & 3 \\
\hline $\mathrm{A}$ & $\mathrm{H}$ & $\mathrm{H}$ & $\mathrm{P}$ & $\mathrm{H}$ & $\mathrm{P}$ & $\mathrm{P}$ & $\mathrm{H}$ & $\mathrm{H}$ & $\mathrm{P}$ & $\mathrm{P}$ & $\mathrm{H}$ & $\mathrm{H}$ & $\mathrm{P}$ & $\mathrm{H}$ & $\mathrm{P}$ & $\mathrm{P}$ & $\mathrm{H}$ & $\mathrm{H}$ & $\mathrm{P}$ & $\mathrm{P}$ & $\mathrm{H}$ & $\mathrm{H}$ & $\mathrm{P}$ & $\mathrm{H}$ & $\mathrm{P}$ & $\mathrm{P}$ & $\mathrm{H}$ & $\mathrm{H}$ & $\mathrm{P}$ & $\mathrm{P}$ \\
\hline $\mathrm{CA}$ & $\mathrm{P}$ & $\mathrm{H}$ & $\mathrm{P}$ & $\mathrm{P}$ & $\mathrm{H}$ & $\mathrm{P}$ & $\mathrm{H}$ & $\mathrm{H}$ & $\mathrm{P}$ & $\mathrm{H}$ & $\mathrm{P}$ & $\mathrm{H}$ & $\mathrm{P}$ & $\mathrm{P}$ & $\mathrm{H}$ & $\mathrm{P}$ & $\mathrm{H}$ & $\mathrm{H}$ & $\mathrm{P}$ & $\mathrm{H}$ & $\mathrm{P}$ & $\mathrm{H}$ & $\mathrm{P}$ & $\mathrm{P}$ & $\mathrm{H}$ & $\mathrm{P}$ & $\mathrm{H}$ & $\mathrm{H}$ & $\mathrm{P}$ & $\mathrm{H}$ \\
\hline $\mathrm{H}$ & $\mathrm{H}$ & $\mathrm{P}$ & $\mathrm{H}$ & $\mathrm{P}$ & $\mathrm{H}$ & $\mathrm{H}$ & $\mathrm{P}$ & $\mathrm{P}$ & $\mathrm{H}$ & $\mathrm{P}$ & $\mathrm{H}$ & $\mathrm{P}$ & $\mathrm{H}$ & $\mathrm{P}$ & $\mathrm{H}$ & $\mathrm{H}$ & $\mathrm{P}$ & $\mathrm{P}$ & $\mathrm{H}$ & $\mathrm{P}$ & $\mathrm{H}$ & $\mathrm{P}$ & $\mathrm{H}$ & $\mathrm{P}$ & $\mathrm{H}$ & $\mathrm{H}$ & $\mathrm{P}$ & $\mathrm{P}$ & $\mathrm{H}$ & $\mathrm{P}$ \\
\hline $\mathrm{D}$ & $\mathrm{P}$ & $\mathrm{P}$ & $\mathrm{H}$ & $\mathrm{H}$ & $\mathrm{P}$ & $\mathrm{H}$ & $\mathrm{P}$ & $\mathrm{P}$ & $\mathrm{H}$ & $\mathrm{H}$ & $\mathrm{P}$ & $\mathrm{P}$ & $\mathrm{H}$ & $\mathrm{H}$ & $\mathrm{P}$ & $\mathrm{H}$ & $\mathrm{P}$ & $\mathrm{P}$ & $\mathrm{H}$ & $\mathrm{H}$ & $\mathrm{P}$ & $\mathrm{P}$ & $\mathrm{H}$ & $\mathrm{H}$ & $\mathrm{P}$ & $\mathrm{H}$ & $\mathrm{P}$ & $\mathrm{P}$ & $\mathrm{H}$ & $\mathrm{H}$ \\
\hline
\end{tabular}

$\mathrm{H}=$ herbal granulas $\mathrm{x}$ first 3 months then placebo $\mathrm{x}$ next 3 months (Month 1,2 \&3 supply herbal granulas, month 4,5 \& 6 , supply placebo)

$\mathrm{P}=$ placebo $\mathrm{x}$ first 3 months then herbal granulas x next 3 months ( Month 1,2 \& 3, supply placebo, Month 4,5 \& 6, supply herbal grannulas)

$\mathrm{H}=$ Group $\mathrm{I}$

$\mathrm{P}=$ Group II 
Appendix E

Study Poster

\section{Chinese Herbal Medicine}

$\boldsymbol{\&}$

\section{Premenstrual Syndrome}

If you have symptoms that worsen during the premenstrual period, you are invited to participate in this study.

The study will involve a

series of consultations and the use of

Chinese herbal granules.

Consultations are available at the St Albans and City Campuses

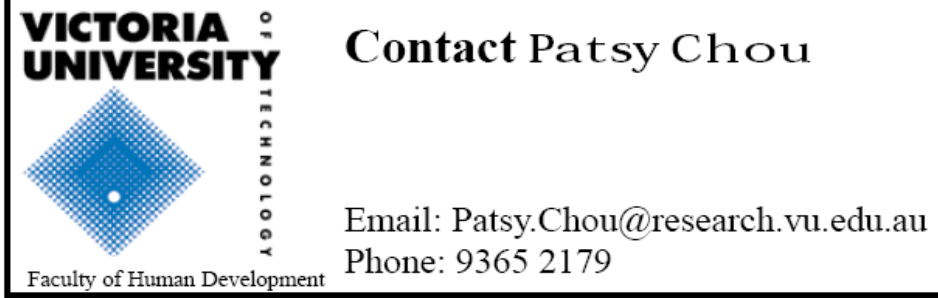


Appendix F

$\underline{\text { Study Flier }}$

\author{
Victoria University \\ School of Health Science
}

\title{
Volunteers Needed
}

For research project:

\section{Chinese Herbal Medicine and Premenstrual Syndrome}

The purpose of this study is to evaluate the efficacy of Chinese herbal medicine for Premenstrual syndrome sufferers in providing symptoms relief. This study involves approximately 7 interviews over a six month period. Free Chinese Medical consultation and Chinese herbal granules will be provided.

\section{For further information contact:}

Patsy Chou

School of Health Science, Victoria University

Mobile Phone: 0403368921

Fax: 98598528

Email: patsy.chou@ research.vu.edu.au 
Appendix G

M.D.Q.

Name

Today's Date

Day of cycle

(Day 1= first day of bleeding)

The following is a list of symptoms which women sometimes experience. For each symptom choose the descriptive category listed below which best describes your experience of that symptom during the week before period. Circle the number of the category which you choose. Even if none of the categories is exactly correct, choose the one that best describes your experience. Please be sure to circle one number for each symptom. Please also remember to put your name and the date in the blank spaces at the top of this page.

Descriptive Categories: 0. No reaction at all. 1. Present, mild. 2. Present, moderate.

3.Present,strong.4.Present,severe.

\begin{tabular}{|c|c|c|c|c|c|c|}
\hline & & \multicolumn{5}{|c|}{ present } \\
\hline & & None & mild & moderate & strong & severe \\
\hline 1 & Muscle stiffness & 0 & 1 & 2 & 3 & 4 \\
\hline 2 & Weight gain & 0 & 1 & 2 & 3 & 4 \\
\hline 3 & Dizziness or faintness & 0 & 1 & 2 & 3 & 4 \\
\hline 4 & Loneliness & 0 & 1 & 2 & 3 & 4 \\
\hline 5 & Headache & 0 & 1 & 2 & 3 & 4 \\
\hline 6 & Skin blemish or disorder & 0 & 1 & 2 & 3 & 4 \\
\hline 7 & Cold sweats & 0 & 1 & 2 & 3 & 4 \\
\hline 8 & Anxiety & 0 & 1 & 2 & 3 & 4 \\
\hline 9 & Cramps & 0 & 1 & 2 & 3 & 4 \\
\hline 10 & Painful or tender breasts & 0 & 1 & 2 & 3 & 4 \\
\hline 11 & Nausea or vomiting & 0 & 1 & 2 & 3 & 4 \\
\hline 12 & Crying & 0 & 1 & 2 & 3 & 4 \\
\hline 13 & Backache & 0 & 1 & 2 & 3 & 4 \\
\hline 14 & Swelling (breasts, abdomen, ankle) & 0 & 1 & 2 & 3 & 4 \\
\hline 15 & Hot flashes & 0 & 1 & 2 & 3 & 4 \\
\hline 16 & Irritability & 0 & 1 & 2 & 3 & 4 \\
\hline
\end{tabular}


$17 \quad$ Fatigue

18 Depression (feeling sad or blue)

19 Mood swings

20 General aches and pains

21 Restlessness

22 Tension

23 Insomnia

24 Poor school or work performance

25 Affectionate

26 Feeling of suffocation

27 Forgetfulness

28 Take naps, stay in bed

29 Orderliness

30 Chest pain

31 Confusion

32 Stay at home from work or school

33 Excitement

$34 \quad$ Ringing in the ears

35 Difficulty concentrating

36 Avoid social activities

$37 \quad$ Feeling of well-being

38 Heart pounding

39 Distractable

40 Decreased efficiency

41 Bursts of energy or activity

42 Numbness or tingling in hands or feet

43 Minor accidents

44 Change in eating habits

45 Blind spots or fuzzy vision

46 Poor motor coordination

47 Poor judgement

$\begin{array}{lllll}0 & 1 & 2 & 3 & 4\end{array}$

$\begin{array}{lllll}0 & 1 & 2 & 3 & 4\end{array}$

$\begin{array}{lllll}0 & 1 & 2 & 3 & 4\end{array}$

$\begin{array}{lllll}0 & 1 & 2 & 3 & 4\end{array}$

$\begin{array}{lllll}0 & 1 & 2 & 3 & 4\end{array}$

$\begin{array}{lllll}0 & 1 & 2 & 3 & 4\end{array}$

$\begin{array}{lllll}0 & 1 & 2 & 3 & 4\end{array}$

$\begin{array}{lllll}0 & 1 & 2 & 3 & 4\end{array}$

$\begin{array}{lllll}0 & 1 & 2 & 3 & 4\end{array}$

$\begin{array}{lllll}0 & 1 & 2 & 3 & 4\end{array}$

$\begin{array}{lllll}0 & 1 & 2 & 3 & 4\end{array}$

$\begin{array}{lllll}0 & 1 & 2 & 3 & 4\end{array}$

$\begin{array}{lllll}0 & 1 & 2 & 3 & 4\end{array}$

$\begin{array}{lllll}0 & 1 & 2 & 3 & 4\end{array}$

$\begin{array}{lllll}0 & 1 & 2 & 3 & 4\end{array}$

$\begin{array}{lllll}0 & 1 & 2 & 3 & 4\end{array}$

$\begin{array}{lllll}0 & 1 & 2 & 3 & 4\end{array}$

$\begin{array}{lllll}0 & 1 & 2 & 3 & 4\end{array}$

$\begin{array}{lllll}0 & 1 & 2 & 3 & 4\end{array}$

$\begin{array}{lllll}0 & 1 & 2 & 3 & 4\end{array}$

$\begin{array}{lllll}0 & 1 & 2 & 3 & 4\end{array}$

$\begin{array}{lllll}0 & 1 & 2 & 3 & 4\end{array}$

$\begin{array}{lllll}0 & 1 & 2 & 3 & 4\end{array}$

$\begin{array}{lllll}0 & 1 & 2 & 3 & 4\end{array}$

$\begin{array}{lllll}0 & 1 & 2 & 3 & 4\end{array}$

$\begin{array}{lllll}0 & 1 & 2 & 3 & 4\end{array}$

$\begin{array}{lllll}0 & 1 & 2 & 3 & 4\end{array}$

$\begin{array}{lllll}0 & 1 & 2 & 3 & 4\end{array}$

$\begin{array}{lllll}0 & 1 & 2 & 3 & 4\end{array}$

$\begin{array}{lllll}0 & 1 & 2 & 3 & 4\end{array}$

$\begin{array}{lllll}0 & 1 & 2 & 3 & 4\end{array}$ 
M.S.D.

Name
Today's Date
Day of cycle
(Day 1 = first day of bleeding)

1.Please complete this form every evening to describe your symptom in the previous 24 hours.

2. Rate each symptom each day.

3.For each symptom choose the descriptive grading list below for grading of symptoms.

4.Fill the number (0-3) of the descriptive grading list which best describes your experience of that symptom of the day in the box of the day of cycle.

5.Start your rating from the $1^{\text {st }}$ day of your cycle (the first day of your full menstruation), and record down the date.

6. When you are menstruating, also rate the condition of the menses, choose the number from the descriptive grading of menses

7.Please turn the page over 
Grading of menses:
0 -none
1-slight 2-moderate

3-heavy

4-heavy and clots

\section{Grading of symptom:}

0 - no symptom

1-mild present but does not interfere with activities

2-moderate-present and interferes with activities but not disabling

3 -severe disabling, unable to function

\begin{tabular}{|l|l|l|l|l|l|l|l|l|l|l|l|l|l|l|l|l|l|l|l|l|l|l|l|l|l|l|l|l|l|l|l|l|l|l|l|l|l|}
\hline $\begin{array}{l}\text { Date of cycle } \\
\text { (Day 1 = First day of bleeding) }\end{array}$ & 1 & 2 & 3 & 4 & 5 & 6 & 7 & 8 & 9 & 1 & 1 & 1 & 1 & 1 & 1 & 1 & 1 & 1 & 1 & 2 & 2 & 2 & 2 & 2 & 2 & 2 & 2 & 2 & 2 & 3 & 3 & 3 & 3 & 3 & 3 & 3 \\
\hline Date & & & & & & & & & & & & & & & & & & & & & & & & & & & & & & & & & & & \\
\hline Menses & & & & & & & & & & & & & & & & & & & & & & & & & & & & & & & & & & \\
\hline
\end{tabular}

\section{Symptoms}

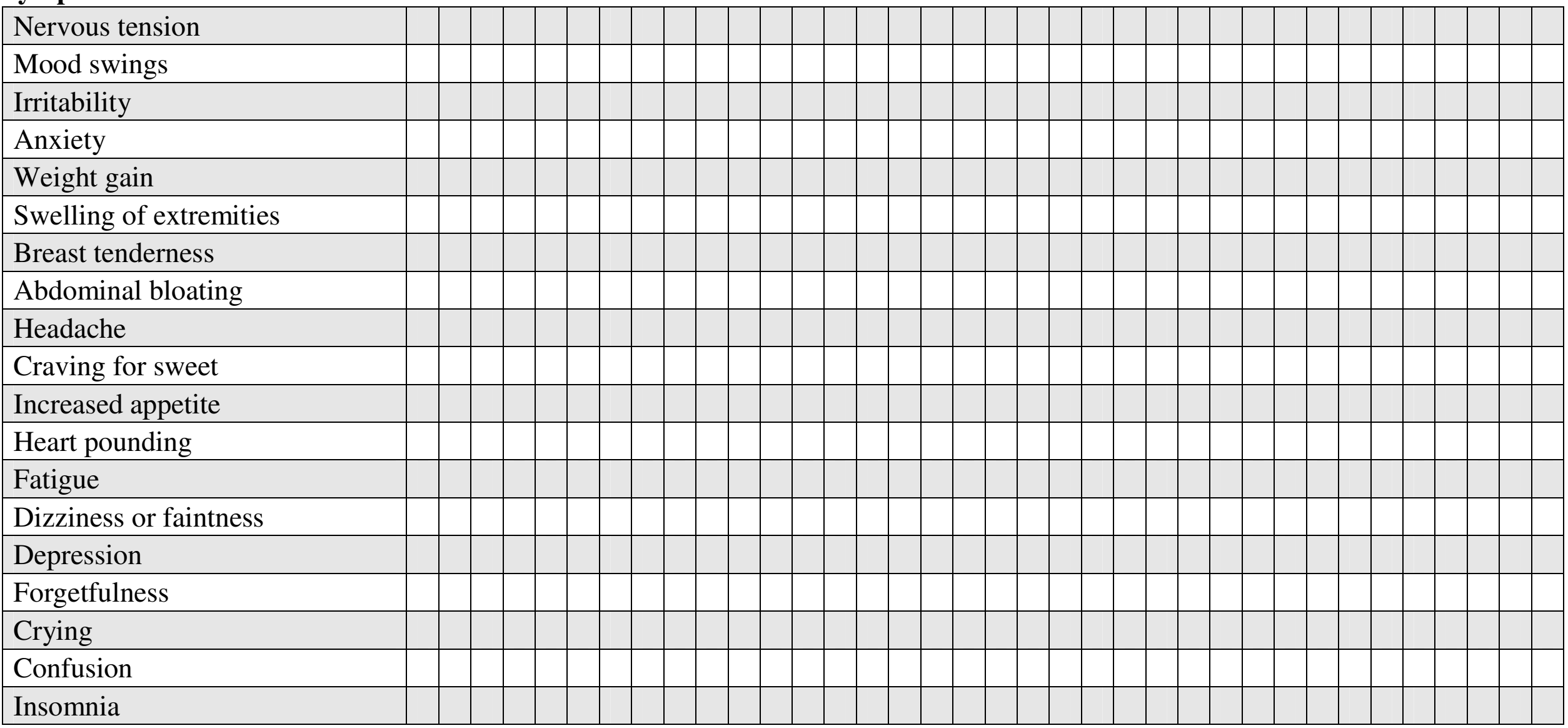




\section{Appendix I}

\section{B.D.I}

Name

Today's Date

Day of cycle

This questionnaire consists of 13 groups of statements. After reading each group of statements carefully, circle the number $(0,1,2$ or 3$)$ next to the one statement in each group which best describes the way you have been feeling the week before the last period. If several statements within a group seem to apply equally well, circle each one. Be sure to read all the statements in each group before making your choice.
A. 0 -I do not feel sad.
1 -I feel sad.
2 -I am sad all the time and I can't snap out of it.
3 -I am so sad or unhappy that I can't stand it.

\section{B. 0 -I am not particularly discouraged about the future. \\ 1 -I feel discouraged about the future. \\ 2 -I feel I have nothing to look forward to. \\ 3 -I feel that the future is hopeless and that things cannot improve.}

C. 0 -I do not feel like a failure.

1 -I feel I have failed more than the 11 average person.

2 -As I look back on my life, all I can see is a lot of failures.

3 -I feel I am a complete failure as a person.

D. 0 -I am not particularly dissatisfied.

1 -I don't enjoy things the way I used to.

2 -I don't get real satisfaction out of anything anymore.

3 -I am dissatisfied or bored with everything

E. 0 -I don't feel particularly guilty.

1 -I feel bad or unworthy a good part of the time.

2 -I feel quite guilty.

3 -I feel as though I am very bad or worthless.

F. 0 -I don't feel disappointed in myself.

1 -I am disappointed in myself .

2 -I am disgusted with myself.

3 -I hate myself.

G. 0 -I don't have any thoughts of killing myself.

1 -I feel I would be better off dead.

2 -I have definite plans about committing suicide.

3 -I would kill myself if I had the chance.
H. 0 -I have not lost interest in other people.

1 -I am less interested in other people than I used to be.

2 -I have lost most of my interest in other people and have little feeling for them.

3 -I have lost all of my interest in other people and don't care about them at all.

I. 0 -I make decisions about as well as ever

1 -I try to put off making decisions.

2 -I have greater difficulty in making decisions than .

3 -I can't make decisions at all anymore.

J. 0 -I don't feel I look any worse than I used to.

1 -I am worried that I am looking old or unattractive.

2 -I feel that there are permanent changes in my appearance that make me look unattractive.

3 -I feel that I am ugly or repulsive-looking.

K. 0 -I can work about as well as before.

1 -It takes an extra effort to get started at doing something .

2 -I have to push myself very hard to do anything. 3 -I can't do any work at all.

L. 0 -I don't get more tired than usual.

1 -I get tired more easily than I used to.

2 -I get tired from doing almost anything.

3 -I am too tired to do anything.

M. 0 -My appetite is no worse than usual.

1 -My appetite is not as good as it used to be.

2 -My appetite is much worse now.

3 -I have no appetite at all anymore. 
Name

Today's Date

Day of cycle

DIRECTIONS: A number of statements that people use to describe themselves are given below. Read each statement and then blacken the appropriate space on the answer sheet to indicate how you felt in the week before the last period. There are no right or wrong answers. Do not spend too much time on anyone statement but give the answer which seems to describe your present feelings best.

1. I feel calm \begin{tabular}{cccc} 
not at all & \multicolumn{2}{c}{ somewhaderately } & \multicolumn{2}{c}{ very } \\
1 & 2 & 3 & much so
\end{tabular}

2. I am furious

3. I am tense

$\begin{array}{llll}1 & 2 & 3 & 4\end{array}$

4. I feel like banging on the table

$\begin{array}{llll}1 & 2 & 3 & 4\end{array}$

5. I feel at ease

$\begin{array}{llll}1 & 2 & 3 & 4\end{array}$

6. I feel angry

$\begin{array}{llll}1 & 2 & 3 & 4\end{array}$

7. I am presently worrying over possible misfortunes

$\begin{array}{llll}1 & 2 & 3 & 4\end{array}$

8. I feel like yelling at somebody

$\begin{array}{llll}1 & 2 & 3 & 4\end{array}$

9. I feel nervous

10. I feel like breaking things

$\begin{array}{llll}1 & 2 & 3 & 4\end{array}$

11. I am jittery

12. I am mad

$\begin{array}{llll}1 & 2 & 3 & 4\end{array}$

13 .I am relaxed

14. I feel irritated

$\begin{array}{llll}1 & 2 & 3 & 4\end{array}$

15. I am worried

$\begin{array}{llll}1 & 2 & 3 & 4\end{array}$

16. I feel like hitting someone

$\begin{array}{llll}1 & 2 & 3 & 4\end{array}$

17. I feel steady

18. I am burned up

$\begin{array}{llll}1 & 2 & 3 & 4\end{array}$

19. I feel frightened

20. I feel like swearing

$\begin{array}{llll}1 & 2 & 3 & 4\end{array}$

$\begin{array}{llll}1 & 2 & 3 & 4\end{array}$

$\begin{array}{llll}1 & 2 & 3 & 4\end{array}$

$\begin{array}{llll}1 & 2 & 3 & 4\end{array}$

$\begin{array}{llll}1 & 2 & 3 & 4\end{array}$

$\begin{array}{llll}1 & 2 & 3 & 4\end{array}$

$\begin{array}{llll}1 & 2 & 3 & 4\end{array}$


Appendix K

P.S.S.

Name

Today's Date

Day of cycle

\section{Circle the response that reflects your reaction}

\begin{tabular}{|c|c|c|c|c|c|}
\hline & Never & $\begin{array}{c}\text { Almost } \\
\text { never }\end{array}$ & Sometimes & $\begin{array}{l}\text { Fairly } \\
\text { s often }\end{array}$ & $\begin{array}{l}\text { Very } \\
\text { often }\end{array}$ \\
\hline $\begin{array}{l}\text { 1.In the last month, how often have you been upset because of } \\
\text { something that happened unexpectedly? }\end{array}$ & 0 & 1 & 2 & 3 & 4 \\
\hline $\begin{array}{l}\text { 2. In the last month, how often have you felt that you were unable } \\
\text { to control the important things in your life? }\end{array}$ & 0 & 1 & 2 & 3 & 4 \\
\hline $\begin{array}{l}\text { 3. In the last month, how often have you felt nervous and } \\
\text { "stressed"? }\end{array}$ & 0 & 1 & 2 & 3 & 4 \\
\hline $\begin{array}{l}\text { 4. In the last month, how often have you dealt successfully with } \\
\text { irritating life hassles? }\end{array}$ & 0 & 1 & 2 & 3 & 4 \\
\hline $\begin{array}{l}\text { 5. In the last month, how often have you felt that you were } \\
\text { effectively coping with important changes that were occurring in } \\
\text { your life? }\end{array}$ & 0 & 1 & 2 & 3 & 4 \\
\hline
\end{tabular}

6. In the last month, how often have you felt confident about your $\quad \begin{array}{lllllll}\mathbf{0} & \mathbf{1} & \mathbf{2} & \mathbf{3} & \mathbf{4}\end{array}$ ability to handle your personal problems?

7. In the last month, how often have you felt that things were going your way?

$\begin{array}{lllllllll}\text { 8. In the last month, how often have you found that you could } & & \mathbf{0} & \mathbf{1} & \mathbf{2} & \mathbf{3} & \mathbf{4}\end{array}$ not cope with all the things that you had to do?

9. In the last month, how often have you been able to control irritations in your life?

10. In the last month, how often have you felt that you were on top $\quad \begin{array}{lllllll}\mathbf{0} & \mathbf{1} & \mathbf{2} & \mathbf{3} & \mathbf{4}\end{array}$ of things?

11. In the last month, how often have you been angered because of $\quad \begin{array}{lllllll}\mathbf{0} & \mathbf{1} & \mathbf{2} & \mathbf{3} & \mathbf{4}\end{array}$ things that happened that were outside of your control?

12. In the last month, how often have you found yourself thinking $\quad \begin{array}{lllllll}\mathbf{0} & \mathbf{1} & \mathbf{2} & \mathbf{3} & \mathbf{4}\end{array}$ about things that you have to accomplish?

13. In the last month, how often have you been able to control the way you spend your time?

14. In the last month, how often have you felt difficulties were piling up so high that you could not overcome them?

$\begin{array}{lllll}\mathbf{0} & \mathbf{1} & \mathbf{2} & \mathbf{3} & \mathbf{4} \\ \mathbf{0} & \mathbf{1} & \mathbf{2} & \mathbf{3} & \mathbf{4}\end{array}$


Appendix L

VICTORIA :

UNIVERSITY

Preliminary Questionnaire

*All information will remain strictly confidential

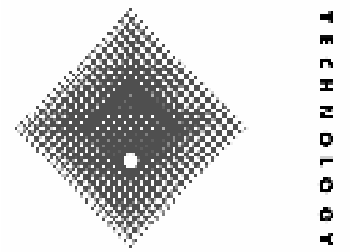

Name

Date

Please answer all items, check those answers that are applicable

1. Name:

2. Address:

3. Tel. No. Home:

Work:

Mobil:

4. Age:

Date of Birth:

5. Marital Status:

6. Occupation:
professional $\square$, small business owner $\square$, technical $\square$,

semi-professional $\square$, clerk or salesperson $\square$, unskilled $\square, \quad$ other

7. Are you currently working?

Yes:

Full Time Part Time No:

8. If you are not currently working, are you

$\begin{array}{ll}\text { unemployed } \square, & \text { retired } \square, \\ \text { student } \square, & \text { other } \square\end{array}$

9.What is the highest level of formal education you have completed?

Secondary school:

TAFE:

University:

Others:

10. What is your country of birth?

11. How long have you been in Australia?

12. What is your culture /ethnic background? (e.g. Greek, Italian ) 


\section{History of Menstruation}

13. Age at first menses:

14. Are your periods regular? (about every 28 days)
Always Usually
Occasionally
Never

15. Have you had regular period in the last 6 months? Yes

N0

16. Number of days of your usual cycle

17. Length of menstruation

18. Amount: Scanty:

Normal:

Heavy:

Very heavy:

19. Colour: Reddish:

Bright red:

Dark red:

Purple:

20. Nature: Thin: Normal: Thick:

21.Any clots: large pieces:

Small pieces:

22. Did you have any menstrual problems (e.g. cramps, heavy bleeding)?

Type of problem:

How long it last?

How bad?

23. Date of last menstruation:

24. Day of cycle now:

\section{Obstetric History}

25. Have you ever been pregnant? Yes:

No:

26.How many? Pregnancies:

Abortion:

Stillbirth:

Children: Male (no. \& age)

Female (no. \& age)

27. What sort of contraception, if any, are you using at the moment?

Oral contraception Tubal Ligation when was it? Vasectomy None Others, please specify

28. Are you pregnant or lactating at the moment? 


\section{Medical History}

29. How is your health, in general?

30. Have you ever been told that you have:

Endometriosis

Fibroids

Breast masses

Thyroid disease

Diabetes

Allergies

Hypertension

31. Have you suffered any major illness in the past? When

32. Please name any gynaecological illness you have?

33. Please name any hormonal problems you have?

34. Please name any psychological or emotional problems you have?

35. Have you had any operations?

36. Are you taking any medicines currently? Pain relieving drugs

Sedatives Tranquillizers

Antidepressants Hormones

Steroids Water pills:

Others:

37. Are you taking Birth control pills

, Vitamins

Calcium supplement Magnesium Supplement

Evening primose oil Chaste tree berries

38. Do you have any history of allergy? Yes

No

If so, what allergic reaction was involved?

39. Have you ever had a reaction to drugs or foods? Yes

No

If so, which drug / food

and what reaction?

40. Do you smoke? If so, how many cigarettes per day?

None 10 or less $10-20$ more than 20

41. Do you drink alcohol? If so, what do you drink?

42. How many glasses per day? None 1-2 glasses 2-3 glasses more than 5 glasses 
43. Have you had Chinese herbal medicine before? Yes

No

44. Which form of Chinese herbal medicine have you had before?

45. What brought you to seek Chinese herbal treatment?

\section{Family History}

46. Does any one else in your family have PMS?

47. Are you happy with your family life? Yes

No

48. Are you happy at work? Yes No

49. Number of siblings: Male Female

Ordinal position

\section{Premenstrual History}

50. Please list your premenstrual symptoms, in order of severity.

51. How do these symptoms interfere with your life?

Slight Moderate

severe Incapacitating

52. Do they occur every month?

53. How long have these symptoms been present?

54. How many days before your period do these symptoms occur?

55. When do these symptoms disappear?

56. Have you had any prior treatment for premenstrual syndrome? Yes No

57. What type of treatment?

Result 


\section{Victoria University}

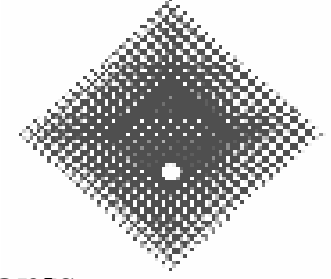

\section{Information Statement for Participants}

\section{$\underline{\text { Chinese Herbal Medicine and Premenstrual Syndrome }}$}

We would like to invite you to participate in a study into the effectiveness of Chinese herbal medicine for the treatment of premenstrual syndrome (PMS) on Australian women. Please read the following information about the study and feel free to ask any question or discuss any concern that you may have.

\section{What the study is about}

A large percentage of women experience severe PMS which can be expected to last until the menopause, therefore any intervention must be effective, safe for long-term use and free from any side effect. However, the current treatment options in Western medicine are either not acceptable to some women or cause unwanted side- effects.

On the other hand, Chinese herbal medicine has been used for thousands of years, and has been claimed to be safe and effective through clinical experience. In view of these facts, the study will investigate the treatment therapy for PMS using Chinese herbal medicine (herbal granules).

This study has been approved by the Human Research Ethics Committee, Victoria University to ensure that the rights of human subjects are protected. Ethical guidelines set out by the National Health and Medical Research Council (NH\&MRC) will be strictly followed.

\section{Purpose of the study}

The aim of this study is

(4) to scientifically evaluate the effectiveness of Chinese herbal medicine for the treatment of PMS within the theoretical frame-work of TCM, and

(5) to evaluate its efficacy on Australian women in providing symptomatic relief.

\section{Procedures to be followed}

To take part in this study you must be a woman who has symptoms and signs which worsen before menstruation and must also:

- Have history of premenstrual complaints for at least six months

- Be aged 18 years or over

- Have been living in Australia for over two years

- Have regular menses, and not be pregnant or lactating

- Be physically healthy

- Have no gynaecological or endocrine illness

- Have no history of psychiatric disorders

- Not currently be using of hormones, antidepressants, tranquillisers, diuretics, hypertension medicine or steroid based anti-inflammatory agents. 
- Have experienced at least $30 \%$ increase in the severity and number of symptoms during the pre-menstrual phase compared to the post-menstrual phase demonstrated in prospective assessments over two consecutive months

- take part in a brief interview to provide your medical and psychosocial history, and PMS symptoms (this will take 10 to 15 minutes), and complete a symptom record using a menstrual diary (MSD) at home for two consecutive menstrual cycles (that will take 2 to 3 minutes each day), and return the completed MSD to the investigator by post.

This study will compare the effect of Chinese herbal granules with an inactive granular powder (placebo) on Premenstrual syndrome. You will be interviewed by the investigator who is a registered Chinese medical practitioner. A diagnosis (TCM pattern of disharmony) according to traditional Chinese medicine will be made. You will then be assigned randomly either to Group I or Group II, and receive either herbal granules for three months then placebo for another three months. This means that you will not know whether the granules you are given during the study will be Chinese herbal granules or powder that looks identical to Chinese herbal granules, but which is inactive. You will have to take these granules three times a day with half a glass of water for a duration of 10 days before each menstruation for six consecutive cycles. The practitioner and investigator also will not know which of the two medicines you have been given.

You will also be interviewed by the investigator every month during your post menstrual period, and be required to complete the menstrual diary for the six menstrual cycles, and a few measures of your moods and symptoms. Each interview will take about 20 minutes, and the questionnaires will take about 10 minutes to complete.

\section{Adverse Effect}

No adverse effects are anticipated as all the herbs to be used are commonly accepted and applied to a wide range of health problems, and are approved by the Therapeutic Goods Administration (TGA) for their safety and quality. The application of holistic care and the use of natural herbs in traditional Chinese medicine are well documented and supported by over 2000 years of clinical practice and experience. Whilst it is unusual for any participant to experience any allergic effect from the herbal granules, the most possible symptoms of allergies that may occur are skin rashes, abdominal discomfort or general malaise.

Some people may find the taste of herbal granules intolerable, e.g. bitter, or pungent taste of the herbs. This undesirable taste can be minimized by putting the herbal granules into capsules, or taking the granules with honey, jam or juice. This after taste effect is only a short-term effect, and it poses no risk to the participants. You are free to withdraw from the study at any time with no penalty and no alternative treatment can be offered to you.

\section{Confidentiality of the records}

Your records that are related to this study will be maintained in the strictest confidentiality. No identity of any specific patient in this study will be disclosed in any public reports or publications. You will be given a participant code number and all the information you provide will be kept with that code. The list of names and codes will be kept separately from your record. No person other than the investigators will have access to your record. All data will be destroyed five years after the completion of the study.

\section{If problems develop}

You should stop the medication, contact the investigator and seek medical advice if condition persists after medication has been discontinued. In the event of an anaphylactic shock, you should seek medical help as soon as possible or call an ambulance. 
If you are concerned about any part of this study, please contact the clinical investigator, Professor Carol Morse, at Victoria University, Footscray Park campus, phone: 96884085.

Financial compensation is not available for medical treatment elsewhere, loss of work, or other expenses.

Additional information

You are encouraged to ask any questions at any time during your participation in the study. You may keep this information statement for future reference.

Any queries about your participation in this study may be directed to the researcher Dr.Hong Xu (TCM), Ph. No.93652765, or Professor Carol Morse, Ph. No. 96884085.

If you have any queries or complaints about the way you have been treated, you may contact Dr. Ilma O'Brien, the Secretary, University Human Research Ethics Committee, Victoria University, Po Box 14428, MCMC Melbourne 8001 (telephone No: 03-9688 4710) 


\section{Appendix N}

\section{Victoria University}

\section{Consent Form for Participation in Research}

\section{INFORMATION TO PARTICIPANTS:}

We would like to invite you to take part in a study into a treatment therapy for Premenstrual Syndrome using Chinese herbal medicine. Please see attachment "Information Statement for Participants" for background information.

\section{CERTIFICATION BY PARTICIPANT}

I......

of.

certify that I am at least 18 years old and that I am voluntarily giving my consent to participate in the research study entitled: "Chinese Herbal Medicine and Premenstrual Syndrome",

being conducted at the Victoria University by Carol Morse, Hong Xu, and Patsy Chou

I certify that I understand all the stated information provided in the Information Statement for Participants (3 pages) of this study and the involvement that I will have in the study, and that the aim and the procedure of the study, the nature of the Chinese medicine, together with any possible risks and safeguards of the project have been fully explained to me by and that I freely consent to participation involving the use on me for this study.

I certify that I have had the opportunity to have any questions answered and that I understand that I can withdraw from the study at any time and that this withdrawal will not jeopardise me in any way. I have been informed that the information I provide will be kept confidential.

Participant:

Signed:

Date:

Witness other than the researcher:

Signed:

Date:

Print Name

Any queries about your participation in this study may be directed to the researcher Dr.Hong Xu (TCM), Ph. No.93652765, or Professor Carol Morse, Ph. No. 96884085.

If you have any queries or complaints about the way you have been treated, you may contact Dr. Ilma O'Brien, the Secretary, University Human Research Ethics Committee, Victoria University, Po Box 14428, MCMC Melbourne 8001 (telephone No: 03-9688 4710) 